2

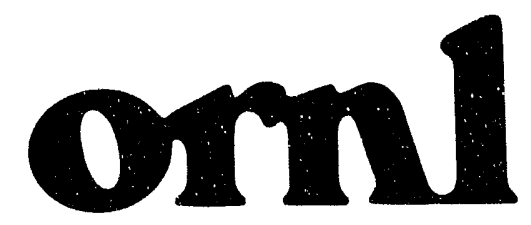

OAK RIDGE NATIONAL LABORATORY

MARTIN MAQTIETRA
1990 NATIONWIDE TRUCK ACTIVITY AND COMMODITY SURVEY SUMMARY REPORT

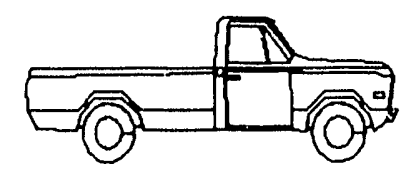

Prepared by

Statistics and Data Analysis Group Center for Transportation Analysis Energy Division

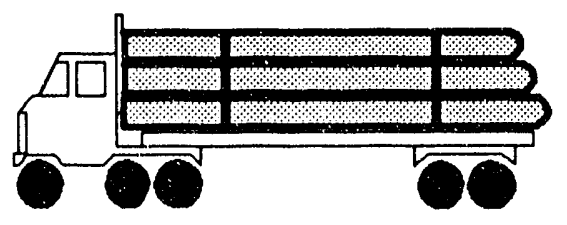

MANAGED BY

MARTIN MARIETTA ENERGY SYSTEMS, INC.

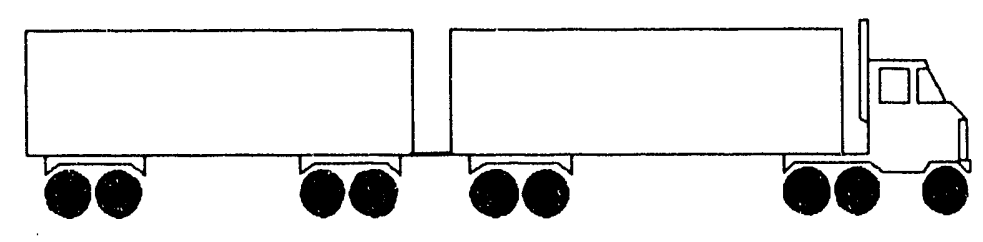


ORNL/TM-12361

\title{
1990 NATIONWIDE TRUCK ACTIVITY AND COMMODITY SURVEY SELECTED TABULATIONS
}

June 1993

\author{
Prepared for \\ Federal Highway Administration \\ U.S. Department of Transportation \\ Washington, D.C. 20590
}

\author{
Prepared by \\ Statistics and Data Analysis Group \\ Center for Transportation Analysis \\ Energy Division \\ Oak Ridge National Laboratory \\ Oak Ridge, Tennessee 37831 \\ managed by \\ MARTIN MARIETTA ENERGY SYSTEMS, INC. \\ for the \\ U.S. DEPARTMENT OF ENERGY \\ under \\ Contract No. DE-AC05-84OR21400

\section{DISCLAIMER}

This report was prepared as an account of work sponsored by an agency of the United States Government. Neither the United States Government nor any agency thereof, nor any of their employees, makes any warranty, express or implied, or assumes any legal liability or responsibility for the accuracy, completeness, or usefulness of any information, apparatus, product, or process disclosed, or represents that its use would not infringe privately owned rights. Referprocess disclosed, or represents that its manufacturer, or otherwise does not necessarily constitute or imply its endorsement, recommanufacturer, or otherwise does not necessarily constitute or any agency thereof. The views mendation, or favoring by the United States Government or any agency thereof. The views
and opinions of authors expressed herein do not necessarily state or reflect those of the United States Government or any agency thereof. 
EXECUTIVE SUMMARY $\ldots \ldots \ldots \ldots \ldots \ldots \ldots \ldots \ldots \ldots$

ACKNOWLEDGMENTS $\ldots \ldots \ldots \ldots \ldots \ldots \ldots \ldots \ldots \ldots$ vii

LIST OF TABLES $\ldots \ldots \ldots \ldots \ldots \ldots \ldots \ldots \ldots \ldots \ldots \ldots \ldots \ldots \ldots$ ix

CHAPTER 1. INTRODUCTION AND OVERVIEW (Table 1.1) $\ldots \ldots \ldots \ldots \ldots 1$

- Background .......................... 2

- 1990 NTACS Universe Description of Four Year and Older Trucks . 3

- Estimated 1990 NTACS Universe Size of Four Year and

Older Trucks ........................ 4

- 1990 NTACS Sample Size of Four Year and Older Trucks . . . . . . 4

- Estimated 1990 NTACS Sample Respondents Reporting Annual and General Data .......................... 4

- Estimated 1990 NTACS Sample Respondents Reporting Sample Day One Data ...........................

- Table Subject Matter Description/Definitions ............6 6

CHAPTER 2. GENERAL DESCRIPTIONS AND ANNUAL ACTIVITIES OF THE 1990 NTACS TRUCK POPULATION (Tables $2.1-2.24 \mathrm{~b}$ ) $\ldots \ldots \ldots 13$

CHAPTER 3. DAILY DESCRIPTIONS AND ACTIVITIES OF THE 1990 NTACS TRUCK POPULATION (Tables 3.1-3.30) . . . . . . . . . 39

CHAPTER 4. UNWEIGHTED NUMBERS ON TRIP-SEGMENTS (Tables 4.1-4.5) 77

CHAPTER 5. ADJUSTED ESTIMATES OF THE 1990 NTACS TRUCK POPULATION (Table 5.1) $\ldots \ldots \ldots \ldots \ldots \ldots \ldots \ldots \ldots$

APPENDIX A. DESIGN OF THE 1990 NATIONWIDE TRUCK ACTIVITY AND COMMODITY SURVEY $\ldots \ldots \ldots \ldots \ldots \ldots \ldots \ldots$ A-1

APPENDIX B. MAP OF FOUR CENSUS REGIONS AND NINE CENSUS DIVISIONS $\ldots \ldots \ldots \ldots \ldots \ldots \ldots \ldots \ldots \ldots \ldots \ldots \ldots \ldots \ldots$

APPENDIX C. THE 1990 NTACS-I QUESTIONNAIRE . . . . . . . . . . . . . C-1 


\section{EXECUTTVE SUMMARY}

The 1990 Nationwide Truck Activity and Commodity Survey (NTACS) provides detailed annual and daily artivity data for a probability sample of trucks responding to the 1987 Truck Inventory an Use Survey (TIUS). The data were collected for days selected at random over a 12-month period ending in October of 1990. Selected results from the 1990 NTACS are presented in this summary report.

Unlike the 1987 TIUS sample which reported only annual data, the 1990 NTACS sample truck operators were asked to report daily as well as annual data in order to capture the temporal and geographic variation in truck use and to measure other detailed attributes of trucking that were beyond the scope of the TIUS. The 1990 NTACS sample of 44,002 trucks was a subsample of the approximately 105,000 sample truck respondents to the 1987 TIUS. Approximately $\llcorner 2,004$ sample truck operators returned the 1990 NTACS questionnaire of which 9,794 operators reported some information on daily truck activity.

The reported data were used to produce extimates for the universe of all four year and older trucks operating in the United States during 1990 which is estimated to be approximately $41,768,000$ trucks. This universe of trucks is referred to as the "1990 NTACS universe." No sample data were collected from trucks less than four years old.

As a first attempt to collect detailed trip characteristics and other information for a large sample of 1987 TIUS respondents, the 1990 NTACS was one of the most ambitious efforts undertaken to measure trucking activity and tackled measurement problems with little previous experience for guidance. Not unlike the initial execution of comparable large-scale nationwide complex data collection efforts, the 1990 NTACS suffered from high nonresponse and data inconsistency problems. Where possible, the collected data were adjusted to compensate for and to decrease the extent of these problems. While these problems have increased the uncenainty of the 1990 NTACS data quality, it was felt that these data could be useful to analysts if uied wth caution and in conjunction with data of known reliability. These data are available on request in the form of a public use file from Oak Ridge National Laboratory.

For easy reference, this report presents selected tables of trucking activity for the 1990 NTACS universe.

Selected highlights include:

- Estimated number of four year and older trucks operating in the United States in 1990 was $41,768,000$ trucks (Chapter 2). This estimate adjusted to include trucks under four years old was $54,112,000$ trucks ( Table 5.1). 
- Estimate of annual vehicle miles traveled (VMT) for the four year and older trucks operating in the United States in 1990 was 440,174,000,000 miles (Chapter 2). This estimate adjusted to include trucks under four years old was $652,147,000,000$ miles (Table 5.1).

- Estimate of daily vehicle miles traveled (DVMT) for the four year and older trucks operating in the United States in 1990 was $1,872,000,000$ miles (Chapter 3).

- Distribution by "truck type" of these estimates for the four year and older trucks operating in the United States in 1990 was:

(Trucks in Thousands/Annual and Daily VMT in Millions)

\begin{tabular}{lrcc}
\hline Truck Type & Trucks & Annual VMT & Daily VMT \\
\hline Pickup & 24,537 & 217,420 & 893 \\
Utility & 12,290 & 123,617 & 493 \\
Small & 2,847 & 25,287 & 149 \\
Large & 1,044 & 20,650 & 103 \\
Truck-Tractors & $\underline{1,050}$ & $\underline{53,200}$ & $\underline{233}$ \\
TOTALS & 41,768 & 440,174 & 1,872 \\
\hline
\end{tabular}

- Distribution by "1987 TIUS Commodity-Carrying Status" of these estimates for four year and older trucks operating in the United States in 1990 was:

(Trucks in Thousands/Annual and Daily VMT in Millions)

\begin{tabular}{lrrr}
\hline Commodity-Carrying Status & Trucks & Annual VMT & Daily VMT \\
\hline Commodity-Carrying & & & \\
- Short Haul & 7,365 & 98,354 & 457 \\
- Long Haul & 766 & 41,292 & 176 \\
Non-Commodity-Carrying & & & \\
- Business Use & 6,393 & 67,385 & 274 \\
- Personal Use & 27,125 & 232,733 & 963 \\
- Not in Use & 119 & -410 & -3 \\
TOTALS & 41,768 & 440,174 & 1,872 \\
\hline
\end{tabular}




\section{IMPORTANT NOTICE}

All estimates in every table of this report have been adjusted using the three stratifying variables: (i) commodity-carrying status, (ii) census division, and (iii) truck type to compensate for extremely high nonresponse rates. (See Tables $G$ and $H$ of Appendix A). As a result, preliminary comparisons with other data sources have revealed inconsistencies -especially when detailed estimates are presented using variables other than the three stratifying variables. Individual estimates are not highly reliable and should be used cautiously only to give impressions of broad trends, patterns, and distributions.

\section{ACKNOWLEDGMENTS}

This report is the result of the efforts of many individuals including Stacy Davis and Pat Hu (both at Center for Transportation Analysis) and Tommy Wright (Mathematical Sciences Section), all of Oak Ridge National Laboratory (ORNL). They received valuable guidance and assistance from William Bostic, Jr., U.S. Bureau of the Census; general direction from Rolf Schmitt and review comments from Charlie Goodman, both at the U.S. Department of Transportation; and review comments from Michael Bronzini, David Greene, Bruce Peterson, and Frank Southworth, all of the Center for Transportation Analysis, ORNL. Finally, the production of this report would have been impossible without the valuable secretarial assistance and patience of Maggie Bruer. 


\section{LIST OF TABLES}

Table Number

Title/Content

Page

NUMBER OF FOUR YEAR AND OLDER TRUCKS AND ASSOCIATED ANNUAL VEHICLE MILES TRAVELED (VMT)

1.1 - BY CENSUS REGION, COMMODITY-CARRYING STATUS, AND

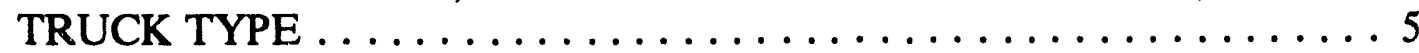

$2.1 \quad$ - BY TRUCK MODEL YEAR $\ldots \ldots \ldots \ldots \ldots \ldots \ldots \ldots \ldots \ldots \ldots$

2.2 - BY TRUCK MODEL YEAR AND COMMODITY-CARRYING

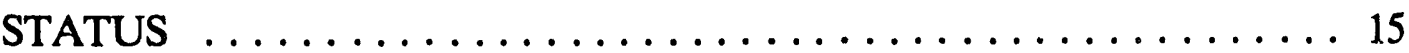

2.3 - BY TRUCK MODEL YEAR AND TRUCK TYPE .......... 16

2.4 - BY COMMODITY-CARRYING STATUS AND TRUCK TYPE $\ldots \ldots .17$

2.5 - BY TRUCK DESCRIPTION AND COMMODITY-CARRYING

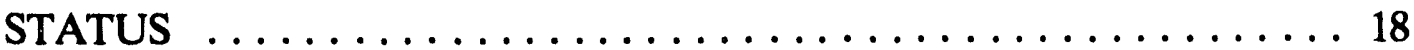

2.6 - BY TRUCK DESCRIPTION AND MAJOR USE $\ldots \ldots \ldots \ldots \ldots \ldots$

$2.7 \quad$ - BY TRUCK CONFIGURATION $\ldots \ldots \ldots \ldots \ldots \ldots \ldots \ldots \ldots 20$

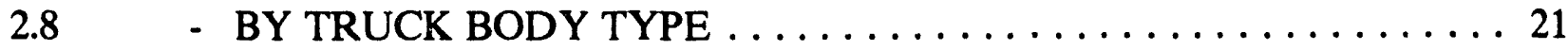

2.9 - BY NUMBER OF STATES OPERATED IN AND CENSUS REGION 22

2.10 - BY NUMBER OF STATES OPERATED IN AND COMMODITYCARRYING STATUS $\ldots \ldots \ldots \ldots \ldots \ldots \ldots \ldots \ldots \ldots \ldots \ldots \ldots$

2.11 - BY NUMBER OF STATES OPERATED IN AND TRUCK TYPE $\ldots . .24$

2.12 - BY NUMBER OF STATES OPERATED IN AND MAJOR USE $\ldots . .25$

2.13 - BY NUMBER OF WEEKS OPERATED AND COMMODITY-

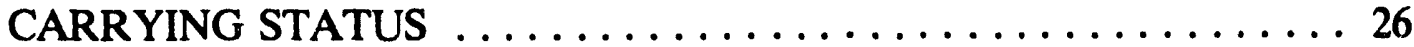

2.14 - BY NUMBER OF WEEKS OPERATED AND TRUCK TYPE . . . . 27

2.15 - BY NUMBER OF WEEKS OPERATED AND MAJOR USE $\ldots \ldots 28$

NUMBER OF FOUR YEAR AND OLDER TRUCKS

2.16 - BY ANNUAL MILES TRAVELED AND TRUCK MODEL YEAR ․ 29

2.17 - BY ANNUAL MILES TRAVELED AND CENSUS REGION ...... 30

2.18 - BY ANNUAL MILES TRAVELED AND COMMODITY-CARRYING

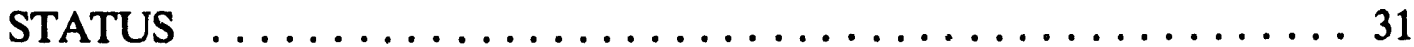

2.19 - BY ANNUAL MILES TRAVELED AND TRUCK TYPE $\ldots \ldots \ldots 32$

2.20 - BY ANNUAL MILES TRAVELED AND MAJOR USE . . . . . 33

2.21 - BY ANNUAL MILES TRAVELED AND TRUCK DESCRIPTION . . 34 
LIST OF TABLES (continued)

Table Number

Title/Content

Page

DISTRIBUTION OF FOUR YEAR AND OLDER TRUCKS THAT OPERATED IN CANADA

2.22a - BY COMMODITY-CARRYING STATUS $\ldots \ldots \ldots \ldots \ldots \ldots \ldots \ldots$

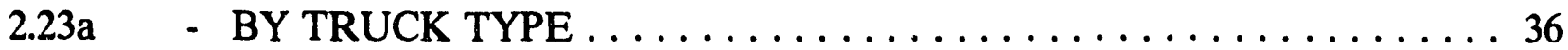

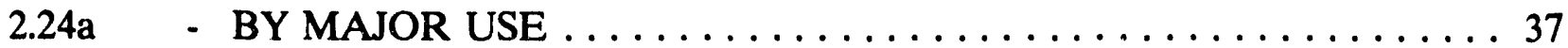

DISTRIBUTION OF FOUR YEAR AND OLDER TRUCKS THAT OPERATED IN MEXICO

2.22b - BY COMMODITY-CARRYING STATUS $\ldots \ldots \ldots \ldots \ldots \ldots \ldots$

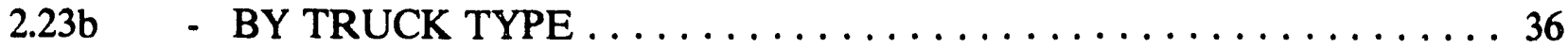

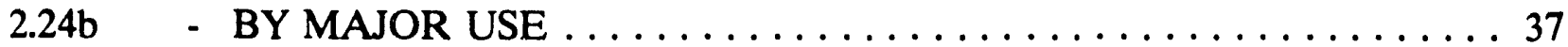

NUMBER OF FOUR YEAR AND OLDER TRUCKS AND ASSOCIATED DAILY VEHICLE MILES TRAVELED (DVMT)

$3.1 \quad$ - BY TRUCK MODEL YEAR ................... 40

3.2 - BY COMMODITY-CARRYING STATUS $\ldots \ldots \ldots \ldots \ldots \ldots \ldots 41$

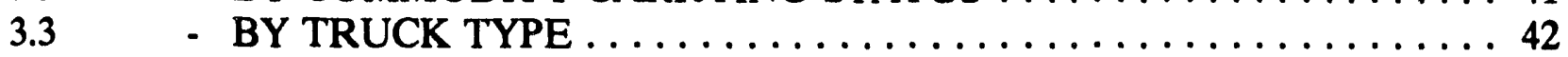

3.4 - BY DAILY TRUCK DESCRIPTION AND DAILY MAJOR USE . . . 43

3.5 - BY DAILY FUEL USE AND DAILY TRUCK DESCRIPTION . . . . 44

3.6 - BY DAILY MAXIMUM EMPTY WEIGHT AND COMMODITY-

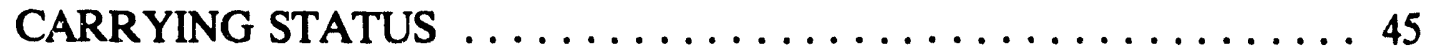

3.7 - BY DAILY MAXIMUM LOADED WEIGHT AND COMMODITYCARRYING STATUS ......................46

3.8 - BY DAILY MAXIMUM PERCENT PAYLOAD SPACE AND

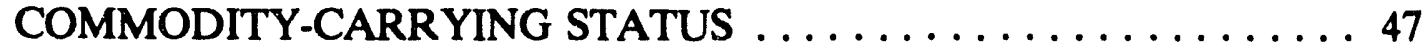

3.9a,b - BY DAILY MAXIMUM EMPTY WEIGHT AND DAILY TRUCK DESCRIPTION ....................... 48,49

3.10a,b - BY DAILY MAXIMUM TRUCK LENGTH AND DAILY TRUCK DESCRIPTION ...................... 50, 51

3.11 - BY DAILY MAXIMUM TRUCK HEIGHT AND DAILY TRUCK DESCRIPTION ........................ 52

3.12 - BY DAILY MAXIMUM PERCENT PAYLOAD SPACE AND DAILY TRUCK DESCRIPTION .................... 53

3.13a,b - BY DAILY MAXIMUM EMPTY WEIGHT AND DAILY MAXIMUM LOADED WEIGHT $\ldots \ldots \ldots \ldots \ldots \ldots \ldots \ldots \ldots \ldots \ldots, 55$ 


\section{LIST OF TABLES (continued)}

Table Number

Title/Content

Page

3.14a,b - BY DAILY MAXIMUM TRUCK LENGTH AND DAILY MAXIMUM LOADED WEIGHT $\ldots \ldots \ldots \ldots \ldots \ldots \ldots \ldots \ldots \ldots \ldots \ldots \ldots$

3.15 - BY DAILY MAXIMUM TRUCK LENGTH AND DAILY MAXIMUM

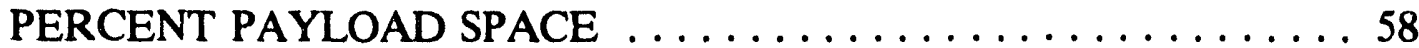

3.16 - BY DAILY COMMODITY CARRIED ................ 59

3.17 - BY DAILY MAJOR USE AND DAILY HAZARDOUS MATERIAL CARRYING STATUS $\ldots \ldots \ldots \ldots \ldots \ldots \ldots \ldots \ldots \ldots$

3.18 - BY DAILY TRUCK DESCRIPTION AND DAILY HAZARDOUS

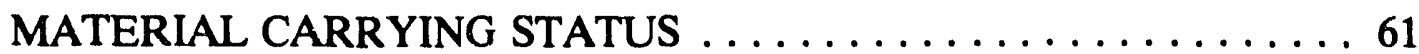

3.19 - BY DAILY PERCENT INTERSTATE, DAILY PERCENT FOUR LANE, DAILY PERCENT OFF ROAD AND DAILY HAZARDOUS MATERIAL CARRYING STATUS $\ldots \ldots \ldots \ldots \ldots \ldots \ldots \ldots 62$

\section{DISTRIBUTION OF FOUR YEAR AND OLDER TRUCKS OVER DAILY TRUCK DESCRIPION}

3.20a - BY DAY OF WEEK OPERATED $\ldots \ldots \ldots \ldots \ldots \ldots \ldots \ldots \ldots \ldots$

DISTRIBUTION OF TOTAL ACTIVITY OF FOUR YEAR AND OLDER TRUCKS THAT OPERATED OVER DAY OF THE WEEK

DISTRIBUTION OF TOTAL ACTIVITY OF FOUR YEAR AND OLDER TRUCKS THAT OPERATED DURING A DAILY TIME PERIOD

- BY TRUCK TYPE .........................665

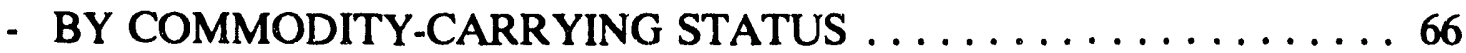

- BY DAILY TRUCK DESCRIPTION . . . . . . . . . . . . . 67

DISTRIBUTION OF FOUR YEAR AND OLDER TRUCKS

3.24 - BY DAILY PERCENT INTERSTATE, DAILY PERCENT FOUR LANE, DAILY PERCENT OFF ROAD, AND COMMODITYCARRYING STATUS $\ldots \ldots \ldots \ldots \ldots \ldots \ldots \ldots \ldots \ldots$

3.25 - BY DAILY PERCENT INTERSTATE, DAILY PERCENT FOUR LANE, DAILY PERCENT OFF ROAD, AND DAILY MAJOR USE . 69 
LIST OF TABLES (continued)

Table Number

Title/Content

Page

3.26 - BY DAILY PERCENT INTERSTATE, DAILY PERCENT FOUR LANE, DAILY PERCENT OFF ROAD, AND DAILY TRUCK

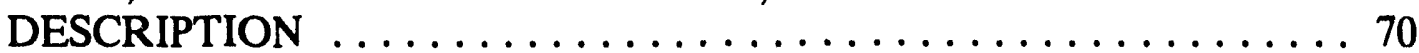

DISTRIBUTION OF FOUR YEAR AND OLDER TRUCKS OVER TRUCK TYPE

3.27a - BY NUMBER OF DAYS OPERATED DURING A WEEK $\ldots \ldots \ldots 71$

DISTRIBUTION OF FOUR YEAR AND OLDER TRUCKS OVER TRUCK MODEL YEAR

3.28a - BY NUMBER OF DAYS OPERATED DURING A WEEK $\ldots \ldots \ldots 73$

DISTRIBUTION OF FOUR YEAR AND OLDER TRUCKS OVER NUMBER OF DAYS OPERATED DURING A WEEK

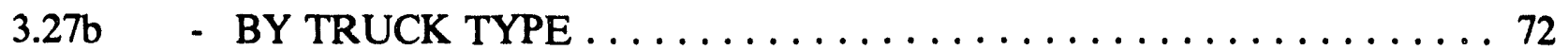

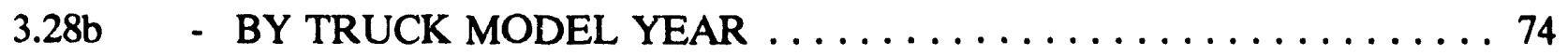

NUMBER OF FOUR YEAR AND OLDER TRUCKS

3.29 - BY ANNUAL MAJOR USE AND DAILY MAJOR USE . . . . . 75

3.30 - BY ANNUAL TRUCK DESCRIPTION AND DAILY TRUCK

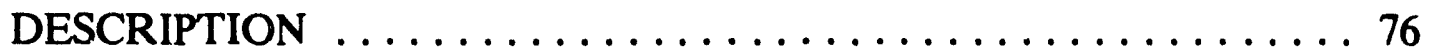

UNWEIGHTED NUMBER OF DAILY TRIP-SEGMENTS FOR FOUR YEAR AND OLDER TRUCKS

4.1 - BY TRIP-SEGMENT LENGTH AND COMMODITY-CARRYING

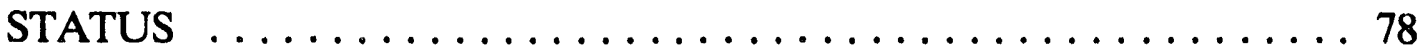

$4.2 \quad-$ BY TRIP-SEGMENT LENGTH AND TRUCK TYPE . . . . . . . . 79 


\section{LIST OF TABLES (continued)}

Table Number

Title/Content

Page

UNWEIGHTED SUMMARY STATISTICS FOR LENGTH OF TRIP SEGMENTS FOR FOUR YEAR AND OLDER TRUCKS

4.3 - BY TRUCK DESCRIPTION FROM 1990 NTACS AND MAJOR USE FROM 1987 TIUS $\ldots \ldots \ldots \ldots \ldots \ldots \ldots \ldots \ldots \ldots \ldots \ldots \ldots \ldots$

UNWEIGHTED NUMBER OF DAILY OPERATING FOUR YEAR AND OLDER TRUCKS

4.4 - BY NUMBER OF DAILY PICKUP/DELIVERY STOPS AND

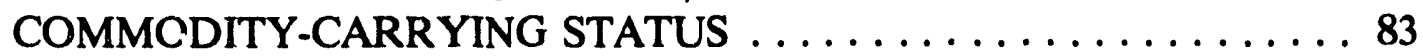

4.5 - BY NUMBER OF DAILY PICKUP/DELIVERY STOPS AND DAILY

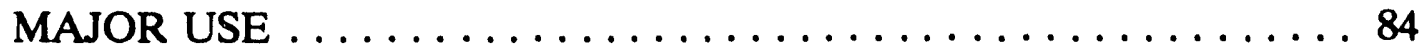

NUMBER (ADJUSTED TO INCLUDE LESS THAN FOUR YEAR OLD) OF TRUCK AND (ADJUSTED) ASSOCIATED ANNUAL VEHICLE MILES TRAVELED (VMT)

5.1 - BY CENSUS REGION, COMMODITY-CARRYING STATUS, AND TRUCK TYPE $\ldots \ldots \ldots \ldots \ldots \ldots \ldots \ldots \ldots \ldots \ldots \ldots . \ldots . \ldots 9$ 


\section{CHAPTER 1}

\section{INTRODUCTION AND OVERVIEW}


The Nationwide Truck Activity and Commodity Survey (NTACS) provides detailed activity data 'or a sample of trucks covered in the 1987 Truck Inventory and Use Survey (TIUS) for days selected at random over a 12-month period ending in 1990. The NTACS was conducted by the U.S. Bureau of the Census for the U.S. Department of Transportation (DOT). A Public Use File for the NTACS was developed by Oak Ridge National Laboratory (ORNL) under a reimbursable agreement with the DOT. The content of the Public Use File and the detailed design of the NTACS are described in the ORNL Report

Technical Documentation for the 1990 Nationwide Truck Activity and Commodity Survey Public Use File. (1992). ORNL Technical Report \#TM-12188, Oak Ridge National Laboratory, Oak Ridge, TN 37831.

The main purpose of this summary report is to provide selected tables based on the public use file.

\section{Background}

The Truck Inventory and Use Survey (TIUS) is the oldest national, vehicle-based survey of freight transportation. The TIUS is performed by the U.S. Bureau of the Census as part of its quinquennial Census of Transportation, and collects extensive information on the typical and year-long use of trucks, vans, and mini-vans. The TIUS sample is drawn from vehicle registration files, and represents all vehicles except buses, automobiles, mobile homes, motorcycles, and vehicles owned by governments. The 1987 TIUS sample includes information on approximately $105,0 C$ ' vehicles.

While the TIUS provides critical information on the composition and typical use of the Nation's trucking fleet, it does not provide a detailed picture of the temporal and geographic variation in truck use. This variation has major implications for multimodal transportation policies involving highway investment requirements, equitable tax policy, effective safety programs, and similar issues. The Federal Highway Administration (FHWA), the Federal Railroad Administration (FRA), and the Office of the Secretary of Transportation (OST) decided to cosponsor a follow-on to the Census Bureau's TIUS in order to capture the temporal and geographic variation in truck use and to measure other detailed attributes of trucking that were beyond the scope of the TIUS. The follow-on became known as the NTACS, and was conducted by the U.S. Bureau of the Census over a 12-month period that ended in 1990 .

The Nationwide Truck Activity and Commodity Survey (NTACS) collected one- and two- day snapshots of a vehicle's activity for a sample of TIUS respondents. The NTACS measured detailed trip characteristics and other information for a large sample of TIUS respondents on randomly sampled days over a 12-month period. The NTACS was designed to provide far more detailed information than is possible on the TIUS. This additional detail is central to understanding the variability in typical vehicle use, relationships between trucking and economic activity, and the role of trucking in intermodal transportation. 
1990 NTACS Universe Description of Four Year and Older Trucks:

The universe for the Nationwide Truck Activity and Commodity Survey (NTACS) is the following:

All trucks operating during the NTACS period (October 29, 1989-October 27, 1990) and registered in one of the 50 states or the District of Columbia on July 1, 1987, and operating in 1987 as estimated by the 1987 Truck Inventory and Use Survey (TIUS). Because the NTACS period covers most of 1990, we refer to this data collection effort as the "1990 NTACS." where:

A clear view of the NTACS universe can be obtained by referring to Figure 1.1

- the 1987 and 1990 U.S. truck populations are represented, respectively, by two overlapping circles;

- the intersection, represented by "A," represents the group of trucks that were in operation in 1987 and that were still in operation in 1990;

- the area that is indicated by "B" represents the group of trucks that was operating in 1987 but which was no longer in operation in 1990, i.e. this group of trucks was scrapped between 1987 and 1990; and

- the area indicated by " $\mathrm{C}$ " represents the group of trucks that began operating after 1987 (more precisely July 1, 1987).

The 1987 truck population consists of trucks in groups A and B; and the 1990 truck population consists of trucks in groups $\mathrm{A}$ and $\mathrm{C}$.

Figure 1.1.

Sampling frames

of TIUS AND NTACS

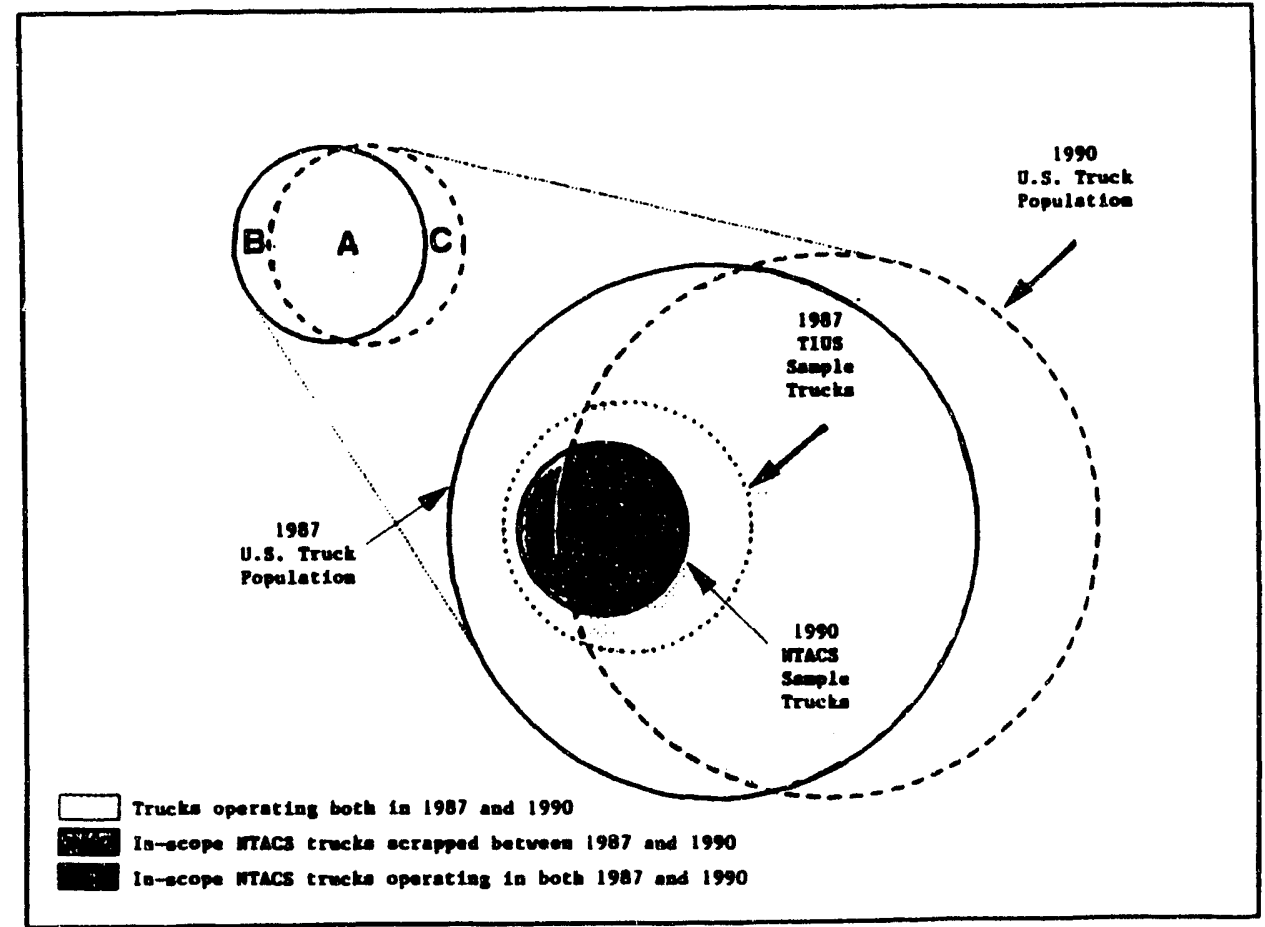


Estimated 1990 NTACS Universe Size of Four Year and Older Trucks:

$41,768,000$ trucks $^{2}$

1990 NTACS Sample Size of Four Year and Older Trucks:

44,002 trucks (See Table E of Appendix A.)

Estimated 1990 NTACS Sample Respondents Reporting Annual and General Data:

22,044 trucks (See Table G of Appendix A.)

Estimated 1990 NTACS Sample Respondents Reporting Sample Day One Data:

9,794 trucks (See Table $H$ of Appendix A.)

The trucks in group A represent what we refer to throughout this report as the "1990 NTACS Univere of Four Year and Older Trucks." All tables in Chapters 2, 3, and 4 are with reference to the 1990 NTACS Universe of Four Year and Older Trucks. Table 1.1 provides an overview of the estimates for the number of trucks and their associated annual vehicle miles traveled for the 1990 NTACS Universe of Four Year and Older Trucks.

In Chapter 5, an attempt is made in Table 5.1 to present "adjusted" estimates for the 1990 U.S. Truck population (A union C) that correspond to those estimates provided in Table 1.1.

\section{IMPORTANT NOTE ON 1990 NTACS DATA QUALITY}

A limited number of data elements from the Census Bureau's 1987 TIUS have been provided on the NTACS Public Use File to merge vehicle characteristics and annual use patterns with trip and shipment characteristics on sampled days. However, the resulting data in the NTACS Public Use File should be used with caution, both because of the file's complexity and because of response rate problems on the NTACS. The complexity of the NTACS contributed to low unit and item response rates. While some data checks, edits, and imputations were done by Census and ORNL, the current data base still includes known inconsistencies. The NTACS data should be only used in conjunction with other data of proven reliability. (For more details, see Section 4 and 5 of the technical documentation for the 1990 Public Use File.) All the tables presented in this report are based on the 1990 NTACS and should therefore be used with caution.

'This estimate is obtained by summing the variable XPFD1 on the public use file. 
Table 1.1

Number of Four Year and Older Trucks and Associated Annual Vehicle Miles Traveled (VMT) by Census-Region, Commodity-Carrying Status, and Truct Type: 1990 NTACS

(Trucks in Thousands/Annual VMT in Millions)

\begin{tabular}{|c|c|c|c|c|c|c|c|c|c|c|c|c|}
\hline \multirow[b]{3}{*}{$\begin{array}{l}\text { Commodity } \\
\text { Carrying } \\
\text { Status }\end{array}$} & \multicolumn{10}{|c|}{ Truck Type } & \multirow{2}{*}{\multicolumn{2}{|c|}{ TOTALS }} \\
\hline & \multicolumn{2}{|c|}{ Pickup } & \multicolumn{2}{|c|}{ Utility } & \multicolumn{2}{|c|}{ Small } & \multicolumn{2}{|c|}{ Large } & \multicolumn{2}{|c|}{ Truck-Tractor } & & \\
\hline & Trucks & Annual & Trucks & $\begin{array}{l}\text { Annual } \\
\text { VMT }\end{array}$ & Trucks & $\begin{array}{l}\text { Annual } \\
\text { VMT }\end{array}$ & Trucks & Annual & Trucks & Annual & Tructes & Annual \\
\hline Commodity-Carrying & & ‘ & & & & & & & & & & \\
\hline -Short Haul & 322 & 3,623 & 291 & 3,243 & 282 & 2,977 & 169 & 2,779 & 90 & 2,682 & 1,153 & 15,303 \\
\hline -Long Haul & 3 & 15 & 16 & 580 & 7 & 81 & 12 & 513 & 46 & 2,928 & 84 & 4,118 \\
\hline \multicolumn{13}{|c|}{ Non-Commodity-Carrying } \\
\hline -Business Use & 441 & 5,403 & 423 & 6,319 & 106 & 592 & 24 & 275 & 7 & 102 & 1,001 & 12691 \\
\hline -Personal Use & 1,937 & 13,294 & 1,283 & 11,736 & 34 & 128 & $\mathbf{0}$ & $\mathbf{0}$ & $\mathbf{a}$ & $\mathbf{a}$ & 3,255 & 25,158 \\
\hline -Not in Use & 0 & $\mathbf{0}$ & 0 & $\mathbf{0}$ & $\mathbf{0}$ & $\mathbf{0}$ & 1 & $\mathbf{a}$ & 0 & 0 & 1 & a \\
\hline Northeand Totals & 2,76 & 22,335 & 2,013 & 21,878 & 430 & 3,778 & 2005 & 3,567 & 143 & 5,712 & 5,493 & 57,270 \\
\hline \multicolumn{13}{|l|}{ Commodity-Carrying } \\
\hline -Short Haul & 848 & 8,310 & 316 & 4,604 & 608 & 4,146 & 279 & 4,886 & 150 & 5,745 & 2,201 & 27,691 \\
\hline -Long Haul & 41 & 1,785 & 35 & 512 & 18 & 149 & 43 & 2,490 & 151 & 12,756 & 208 & 17,692 \\
\hline \multicolumn{13}{|c|}{ Non-Commodity-Carrying } \\
\hline -Business Use & 985 & 10,055 & 446 & 5,619 & 155 & 872 & 36 & 492 & 9 & 327 & 1,630 & 17,366 \\
\hline -Personal Use & 4,001 & 39,687 & 2,230 & 19,252 & 110 & 451 & 15 & 31 & 1 & 2 & 6,357 & 59,123 \\
\hline -Not in Use & $\mathbf{0}$ & $\mathbf{0}$ & $\mathbf{0}$ & 0 & 10 & 36 & $\mathbf{0}$ & 0 & 2 & 14 & 12 & 50 \\
\hline Midumet Totals & 5,875 & 59,838 & 3,026 & 29,987 & 901 & 5,655 & 373 & 7899 & 313 & 18844 & 10,489 & 120,202 \\
\hline \multicolumn{13}{|l|}{ Commodity-Carrying } \\
\hline -Shon Haul & 1,132 & 9,477 & 376 & 4,321 & 579 & 7,329 & 223 & 4,701 & 222 & 7,501 & 2,532 & 33,328 \\
\hline -Long Haul & 43 & 199 & 50 & 337 & 19 & 350 & 35 & 1,601 & 127 & 10,932 & 275 & 13,419 \\
\hline \multicolumn{13}{|c|}{ Non-Commodity-Carrying } \\
\hline -Business Use & 1,271 & 15,561 & 704 & 5,469 & 250 & 1,503 & 32 & 466 & 29 & 588 & 2,287 & 23,587 \\
\hline -Personal Use & 6,949 & 60,547 & 3,029 & 33,389 & 127 & 509 & 35 & 142 & 9 & 54 & 10,150 & 94641 \\
\hline- Not in Use & 24 & 17 & 2 & 15 & 18 & 72 & 4 & 17 & 3 & 19 & $\mathbf{s 1}$ & 140 \\
\hline Sonth Toten & 9,420 & 85,800 & 4,162 & 13,532 & 993 & 9,764 & 330 & 6,926 & 390 & 19,095 & 15,295 & 165,116 \\
\hline \multicolumn{13}{|l|}{ Commodity-Carrying } \\
\hline -Short Haul & 647 & 7,072 & 265 & 4,485 & 336 & 4,477 & 106 & 1,723 & 126 & 4,275 & 1,479 & 20,032 \\
\hline -Long Haul & 28 & 517 & 2 & 5 & 13 & 61 & 15 & 434 & 60 & 5,045 & 119 & 6,063 \\
\hline \multicolumn{13}{|c|}{ Non-Commodity-Carrying } \\
\hline -Busines Use & 966 & 9,091 & 393 & 3,202 & 87 & 1,134 & 13 & 97 & 16 & 217 & 1,475 & 13,741 \\
\hline -Personal Use & 4,877 & 32,615 & 2,421 & 20,503 & 61 & 384 & 3 & 4 & 1 & 5 & 7,363 & $\mathbf{5 3 , 5 1 1}$ \\
\hline -Not in Use & 21 & 153 & 7 & 26 & 26 & 34 & 0 & 0 & 1 & 7 & 55 & 200 \\
\hline Weat Totsh & 6,539 & 49,447 & 3,089 & 28,200 & 523 & 6,091 & 137 & 2,258 & 204 & 9,549 & 10,491 & 95,566 \\
\hline \multicolumn{13}{|l|}{ Commodity-Carrying } \\
\hline -Shorn Haul & 2,948 & 28,482 & 1,247 & 16,652 & 1,805 & 18,929 & 777 & 14,089 & 588 & 20,203 & 7,365 & 98,354 \\
\hline -Long Haul & 115 & 2,516 & 103 & 1,435 & 58 & 642 & 105 & 5,038 & 385 & 31,662 & 766 & 41,292 \\
\hline \multicolumn{13}{|c|}{ Non-Commodity-Carrying } \\
\hline -Business Use & 3,664 & 40,111 & 1,966 & 20,609 & 599 & 4,102 & 104 & 1,329 & 60 & 1,234 & 6,393 & 67,385 \\
\hline -Personal Use & 17,765 & 146,142 & 8,963 & 84,880 & 332 & 1,473 & 54 & 177 & 11 & 62 & 27,125 & 232,733 \\
\hline -Not in Use & 45 & 170 & 10 & 41 & 53 & 142 & 5 & 17 & 6 & 39 & 119 & 410 \\
\hline $\begin{array}{l}\text { UNTED STATES } \\
\text { TOTALS }\end{array}$ & 24,537 & 217,420 & 12,290 & 123,617 & 2847 & 25,287 & 1,044 & 206650 & 1,050 & 53,199 & 41,768 & 40,174 \\
\hline
\end{tabular}

(a) Quantity rounded to zero. 
Table Subject Matter Description/Definitione:

An overview of the content of the tables, how the contents are presented, and the corresponding table numbers are given below. All table values are catimates, and are subjoct to sampling and nonampling errors.

How Presenter

Table Number

NUMABR OF FOUR YEAR AND OLDER TRUCKS AND ASSOCATTED ANNUAL VEHICLB MILES TRAVEI ED BY:

-Census Region ............. 1.1; 2.9

.Commodity-Carrying Status ....... 1.1; 2.2; 2.4

$2.5 ; 2.10 ; 2.13$

-Truck Type . . . . . . . . . . . 1.1; 2.3; 2.4

$2.11 ; 2.14$

-Truck Model Year . . . . . . . . . 2.1; 2.2; 2.3

-Truck Description . . . . . . . . . . 2.5; 2.6

-Major Use ................ 2.6; 2.12; 2.15

-Truck Configuration . . . . . . . 2.7

-Truck Body Type ........... 2.8

- Number of States Operated In . . . . 2. 29; 2.10; $2.11 ; 2.12$

-Number of Weeks Operated . . . . . . 2.13; 2.14; 2.15

NUMBRR OF FOUR YEAR AND OLDER TRUCKS BY:

-Annual Mile Traveled $\ldots \ldots \ldots \ldots .2 .16 ; 2.17 ; 2.18$

$2.19 ; 2.20 ; 2.21$

-Truck Model Year .......... 2.16

-Census Region .............. 2.17

-Commodity-Carrying Status ...... 2.18

-Truck Type . . . . . . . . . . . . . 2.19

-Major Use . . . . . . . . . . . . 2.20

-Truck Deacription . . . . . . . . 2.21

DISTRIBUTION OF FOUR YEAR AND OLDER TRUCKS THAT OPERATED IN CANADA (MEXICO) BY:

-Commodity-Carrying Status . . . . . 2.22a (2.22b)

-Truck Type . . . . . . . . . . . . . . . 2.23a (2.23b)

-Major Use . . . . . . . . . . . 24a (2.24b)

NUNARB OF FOUR YEAR AND OLDER TRUCAKS AND ASSOCATED DAILY VERICIE MIILS

TRAVR BD BY:

-Truck Model Year ............ 3.1

.Commodity-Carrying Status $\ldots \ldots \ldots .3 .2 ; 3.6 ; 3.7 ; 3.8$

-Truck Type ............... 3.3

-Daily Truck Deacription ......... 3.4; 3.5; 3.9; 3.10;

$3.11 ; 3.12 ; 3.18$

Daity Major Use . . . . . . . . . . 3.4; 3.17

-Daily Fued Une . . . . . . . . . . . . 3.5

-Daily Mra Empty Weight . . . . . . . 3.6; 3.9; 3.13

-Daily Max Loaded Weight . . . . . . . . 3.7; 3.13; 3.14

-Daily Max \% Payloed Space . . . . . . . 3.8; 3.12; 3.15

-Daily Max Truct Length . . . . . . . . . 3.10; 3.14; 3.15

-Daily Max Truck Height ......... 3.11

-Daily Commodity Carried ......... 3.16

-Daily Hazardous Material

Carrying Status ............. 3.17; 3.18; 3.19

-Daily \% Intentate ........... 3.19

-Daily \% Four Lane . . . . . . . . . . 3.19

.Daily \% Off Roed ............. 3.19

DISTRIBUTTON OF FOUR YEAR AND OLDER TRUCKS OVER DAILY TRUCK DESCRIPTON BY: -Day of Week Operated .......... 3.20

DISTRIBUTION OF TOTAL ACTTVTYY OF FOUR YEAR AND OLDER TRUCKS THAT OPERATED OYER DAY OF WEEX BY:

-Daily Truck Description ......... 3.20b
How Presented Table Number

DISTRIBUTION OF TOTAL ACTTVITY OF FOUR YRAR AND OLDER TRUCKS THAT OPERATED DURING A DAILY TTME PERIOD BY:

-Truck Type ............. 3.21

-Commodity-Carrying Status . . . . . . 3.22

-Daily Truck Deacriftion ......... 3.23

DISTRIBUTYON OF FOUR YEAR AND OLDER

TRUCKS BY:

-Daily \% Interstate . . . . . . . . . 3.24; $3.25 ; 3.26$

-Daily \% Four Lane . . . . . . . . . . . 3.24; 3.25; 3.26

-Daily \% Off Rosd . . . . . . . . . . . . 3.24; 3.25; 3.26

-Commodity-Carnying Status . . . . 3.24

-Daily Major Use . . . . . . . . . . 3.25

-Daity Truck Deacription .......... 3.26

DISTRIBUTION OF FOUR YEAR AND OLDBR

TRUCKS OVER TRUCX TYPE BY:

- Number of Days Operated During a Week . . 3.27a

DISTRIBUTION OF FOUR YEAR AND OLDER TRUCKS OVER TRUCK MODEL YEAR BY:

-Number of Days Operated During a Week . . 3.28 a

DISTRIBUTION OF FOUR YEAR AND OLDER TRUCGS OVER NUMAER OF DAYS OPERATED DURING A WERK BY:

-Truck Type ............... 3.27b

-Truck Model Year . . . . . . . . . . 3.28b

NULARR OF FOUR YEAR AND OLDER TRUCKS BY: (Annusi) Major Use and Daily

Major Use . . . . . . . . . . . . 3.29

-(Annual) Truck Deacription and

Daily Truck Description ....... 3.30

UNWEIGHIED NUMAER OP DAILY TRIP-SBGMANTS FOR FOUR YEAR AND OLDRR TRUCKS BY:

-Trip-Segment Length .......... 4.1; 4.2

-Commodity-Carrying Status ......4.1 4.1

-Truck Type . . . . . . . . . . . 4.2

UNWBUGHTE SUMMARY STATSTICS POR LBNGTH OP TRIP SBGMRNIS FOR FOUR YEAR AND OLDER TRUCXs BY:

-Truct Description ............ 4.3

-Major Use (from 1987 TIUS) . . . . . 4.3

UNWEIGHTED NUMAER OF DAILY OPERATING FOUR YEAR AND OLDER TRUCKS BY:

- Number of Daily

Pickup/Delivery Stope .......... 4.4; 4.5

-Commodity-Carrying Status ...... 4.4

-Daily Major Use . . . . . . . . . . . 4.5

NUMRER (ADUSTED TO INCUUDE LESS THAN FOUR YEAR OLD) OF TRUCKS AND (ADUSTED) ASSOCIATED ANNUAL VEHICLE MIIES TRAVEI ED BY:

-Census Region ............. 5.1

-Commodity-Carrying Status ....... 5.1

-Truck Type . . . . . . . . . . 5.1 


\section{Content of Tables}

-- Number of four year and older trucks and associated annual vehicle miles traveled:

- The number of four year and older trucks estimates were generated using a daily sampling weight (XPFD1) which has been adjusted for unit nonresponse for daily activity.

- The associated annual vehicle miles traveled estimates were generated using a daily sampling weight (XPFD1) and an annualized reported mileage (ARMIL) which was based on the reported sample day mileage (RMIL1).

-- Distribution of four year and older trucks that operated in Canada (Mexico):

- The distribution percent estimates are based on the number of four year and older trucks estimates.

-- Number of four year and older trucks and associated daily vehicle miles traveled:

- The assosiated daily vehicle miles traveled estimates were generated using a daily sampling weight (XPFD1) and the reported sample day mileage (RMIL1).

-- Distribution of four year and older trucks that operated on a day of the week:

- The distribution percent estimates are based on the number of four year and older trucks estimates. Each truck operator was asked to indicate all days of operation for the week containing the sample day.

-- Distribution of total activity of four year and older trucks that operated during a daily time period:

- The distribution percent estimates are based on the number of four year and older trucks estimates. Each truck operator was asked to indicate whether or not the sample truck operated during each of eight different time periods on the sample day.

b For more details, see the 1990 NTACS Public Use File Technical Documentation. The abbreviations (XPFD1, ARMIL, and RMIL1) refer to the data items on the 1990 NTACS Public Use File which were used to generate most of the data presented in the summary tables in the report. 
-- Distribution of four year and older trucks:

- The distribution percent estimates are based on the number of four year and older trucks estimates.

-- Unweighted number of daily trip-segments for four year and older trucks:

- The unweighted numbers are counts of responding sample trucks only with sample day data. A daily trip-segment is the section of road traveled between one reported stop and the next reported stop on a truck's sample day.

-- Unweighted summary statistics for length of trip-segments for four year and older trucks:

- The unweighted summary statistics give number of trip-segments with some travel and associated mileage statistics by major use and truck description.

-- Unweighted number of daily operating four year and older trucks:

- The unweighted numbers are counts for responding sample trucks only with sample day data.

-- Number (adjusted to include less than four year old) of trucks and (adjusted) associated annual vehicle miles traveled:

- See discussion in Chapter 5.

\section{Data Items Used to Present Table Content}

I. Data Items Reflecting Annual Truck Characteristics and Activity

-- Annual Miles Traveled: Annual miles traveled categories as displayed in the first column of Tables 2.16, 2.17, 2.18, 2.19, 2.20, and 2.21 are based on reported annual miles during the annual period as reported in the 1990 NTACS. Because the data item "Annual Miles Traveled" (RANMI) was available only in categories, it was necessary to produce the "annualized reported mileage (ARMIL) as noted above under Content of Tables. The annual mileage estimates presented "in" the tables are all based on ARMIL, and the only use of the reported annual mileage categories is that in dividing column one of Tables 2.16, 2.17, 2.18, 2.19, 2.20, and 2.21. A contingency table analysis indicated an extremely high correlation between the data items ARMIL and RANMI.

-- Census Region: A map of the 4 census regions is given in Appendix B. A truck's census region designation is based on its state of registration as reported in the 1987 TIUS. 
-- Commodity-Carrying Status: Commodity-carrying status is used to distinguish between commodity-carrying and non-commodity-carrying trucks as identified in the 1987 TIUS. Commodity-carrying trucks are subclassified as short haul or long haul while non-commodity-carrying trucks are subclassified as personal transportation, business use, or not in use. Not in use means a truck was idle, wrecked or otherwise not in use for more than 90 days during 1987. The categories as provided on the public use file are:

1. commodity-carrying, short haul;

2. commodity-carrying, long haul;

3. non-commodity-carrying, personal transportation;

4. non-commodity-carrying, business use; and

5. non-commodity-carrying, idle.

-- Major Use: Major use describes how (personal, contract, common, other) the truck was most frequently operated during the annual period as reported in the 1990 NTACS. In Tables 3.29 and 3.30, this is the same as Annual Major Use.

- Number of States Operated In: Number of states operated in indicates the number of states in which the vehicle operated during the annua! period as reported in the 1990 NTA.CS.

-- Number of Weeks Operated: Number of weeks operated indicates the number of weeks that the vehicle was operated during the annual period as reported in the 1990 NTACS.

-- Truck Body Type: Truck body type indicates the body type that most closely resembles the vehicle during the annual period as reported in the 1990 NTACS.

-- Truck Configuration: Truck configuration indicates the kind(s) of trailer(s) pulled by the truck during the annual period as reported in the 1990 NTACS.

-- Truck Description: Truck description describes the truck as most frequently operated during the annual period as reported in the 1990 NTACS. In Tables 3.29 and 3.30, this is the same as Annual Truck Description.

-- Truck Model Year: Truck model year is as reported in the 1987 TIUS.

-- Truck Type: Truck type refers to the five 1987 TIUS sample strata. The categories as provided on the file are:

1. Pickup trucks.

2. Utility trucks include vans, panel trucks, utilities, jeeps, and station wagons.

3. Small trucks (GVW less than or equal to 26,000 pounds) not classified above.

4. Large trucks (GVW greater than 26,000 pounds) excluding truck-tractors.

5. Truck-tractors. 
II. Data Items Reflecting Daily Truck Characteristics and Activity as Reported on Each Truck's Sample Day One.

-. (Annual) Major Use: (Annual) Major Use is the same as Major Use.

-- (Annual) Truck Description: (Annual) Truck description is the same as Truck Description.

-- Daily Commodity Carried: Daily commodity carried gives the commodity carried with the greatest ton miles by the truck on its sample day one.

-- Daily Fuel Use: Daily fuel use gives the reported amount of fuel used by the truck during its sample day one.

-- Daily Hazardous Material Carrying Status: Daily hazardous material carrying status indicates whether or not the vehicle was used to carry hazardous materials in quantities large enough to require a special placard under the code of federal regulations, Title 49, Transportation, on its sample day one.

-- Daily Major Use: Daily major use describes how (personal, contract, common, other) the truck was most frequently operated during its sample day one.

-- Daily Maximum Empty Weight: Daily maximum empty weight gives the maximum tare (empty) weight of the truck on its sample day one.

-- Daily Maximum Loaded Weight: Daily maximum loaded weight gives the maximum loaded weight of the vehicle (weight of truck and cargo) on its sample day one.

-- Daily Maximum Percent Payload Space: Daily maximum percent payload space gives the maximum space utilized when the vehicle was at its maximum weight on its sample day one.

-- Daily Maximum Truck Height: Daily maximum truck height gives the maximum height of the vehicle when the vehicle was at its maximum weight on its sample day one.

-- Daily Maximum Truck Length: Daily maximum truck length gives the maximum length of the vehicle, from the front bumper to the end of the last trailer when the vehicle was at its maximum weight on its sample day one.

-- Daily Percent Four Lane: Daily percent four lane gives, by categories, the percent of the mileage driven on roads which had four or more lanes but were not on the interstate highway systems by the trucks on its sample day one. 
-- Daily Percent Interstate: Daily percent interstate gives, by categories, the percent of the mileage driven on the interstate highway systems by the truck on its sample day one.

-- Daily Percent Off Road: Daily percent off road gives, by categories, the percent of the mileage driven on off roads (little travel on public roads) by the truck on its sample day one.

-- Daily Truck Description: Daily truck description describes the truck as most frequently operated during its sample day one.

-- Number of Daily Pickup/Delivery Stops: Number of daily pickup/delivery stops is the total number of stops for which the truck's purpose was to pick up or deliver items on its sample day one.

-- Number of Days Operated a Week: Number of days operated a week indicates the number of days of operation for the week containing the truck's sample day one.

-- Thip-Segment Length: Trip-segment length is the distance traveled on a trip segment. 


\section{CHAPTER 2}

\section{GENERAL DESCRIPTIONS AND ANNUAL ACTIVITIES OF THE 1990 NTACS TRUCK POPULATION}

All of the annual VMT estimates in Chapter 2 are based on daily VMT data which have been "annualized." For details on the precise annualization methodology, refer to Section 4 of the Technical Documentation for the 1990 Nationwide Truck Activity and Commodity Survey Public Use File.

$$
\left(\begin{array}{c}
\text { The } \\
\text { Truck's } \\
\text { Annual } \\
\text { VMT }
\end{array}\right)=\left(\begin{array}{c}
\text { The Truck's Reported } \\
\text { Number of Days Operated } \\
\text { During the Week of } \\
\text { Its Sample Day }
\end{array}\right) \times\left(\begin{array}{c}
\text { The Truck's } \\
\text { Reported Number of } \\
\text { Weeks Operated } \\
\text { During the Year }
\end{array}\right) \times\left(\begin{array}{c}
\text { The Truck's } \\
\text { Sample Day } \\
\text { VMT }
\end{array}\right) \text {. }
$$

The key annual VMT estimates throughout Chapter 2 are obtained by expanding the sample values

$$
\left(\begin{array}{c}
\text { The } \\
\text { Truck's } \\
\text { Anumal } \\
\text { VMT }
\end{array}\right) \text {. }
$$

The key daily VMT estimates throughout Chaper 3 are obtained by expanding the sample values

$$
\left(\begin{array}{c}
\text { The Truck's } \\
\text { Sample Day } \\
\text { VMT }
\end{array}\right) \text {. }
$$

\section{DMPORTANT NOTICE}

All estimates in every table of this report have been adjusted using the three stratifying variables: (i) commodity-carrying status, (ii) census division, and (iii) truck type to compensate for extremely high nonresponse rates. (See Tables $\mathrm{G}$ and $\mathrm{H}$ of Appendix A). As a result, preliminary comparisons with other data sources have revealed inconsistencies -especially when detailed estimates are presented using variables other than the three stratifying variables. Individual estimates are not highly reliable and should be used cautiously only to give impressions of broad trends, patterns, and distributions. 
Table 2.1

Number of Four Year and Older Tructs and Associated Annual Vehicle Miles Traveled (VMT) by Truck Model Year: 1990 NTACS

(Trucks ifr Thousands/Annual VMT in Millions)

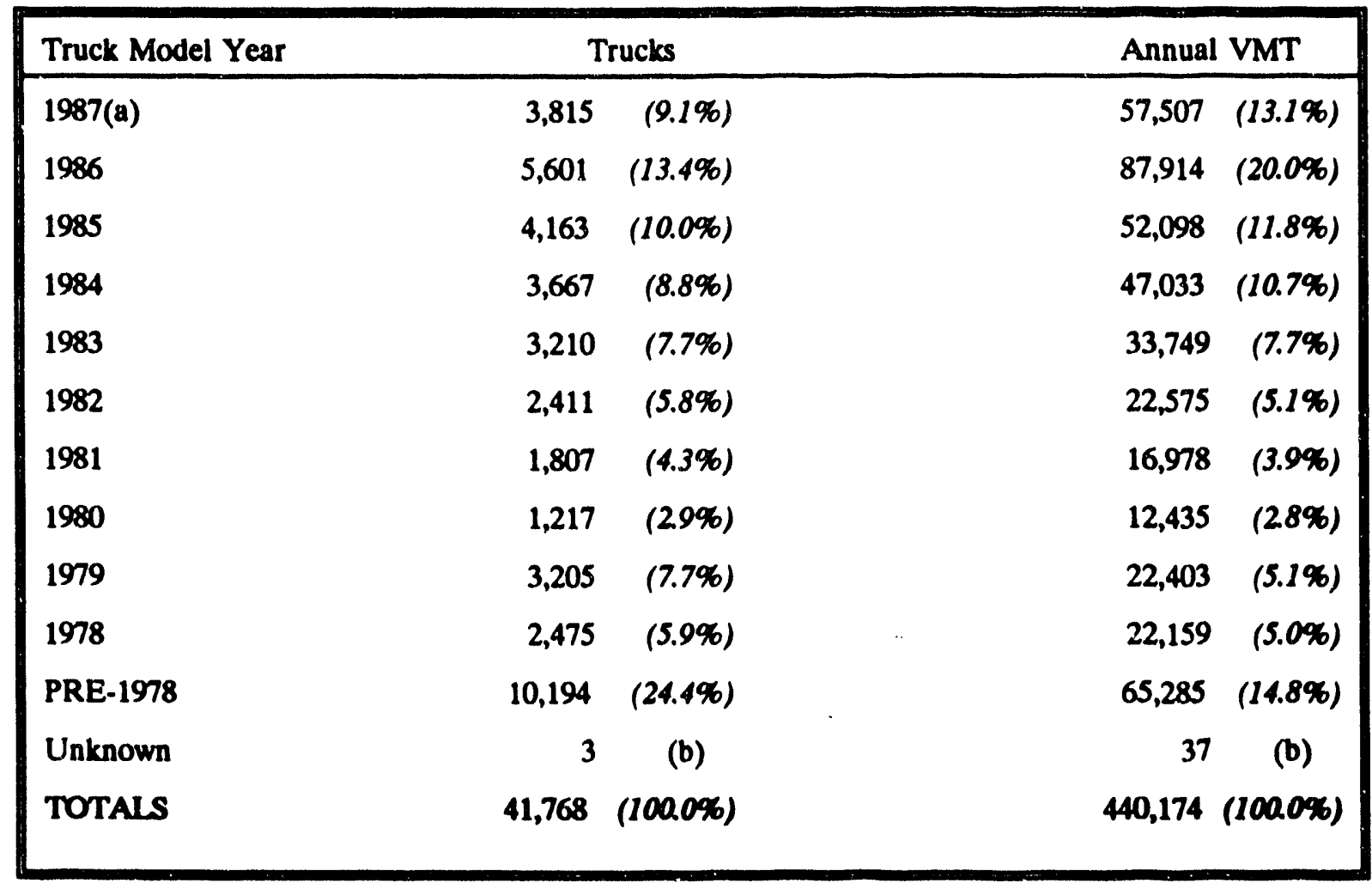

(a) Includes some 1988 models.

(b) Quantity rounded to zero. 
Table 2.2

Number of Four Year and Older Trucks and Assor "ted Annual Vehicle Miles Traveled (VMT) by Truck Model Year and Commodity-Carrying Status: 1990 NTACS

(Trucks in Thousands/Annual VMT in Millions)

\begin{tabular}{|c|c|c|c|c|c|c|c|c|c|c|c|c|}
\hline \multirow[b]{4}{*}{$\begin{array}{l}\text { Truck } \\
\text { Model } \\
\text { Year }\end{array}$} & \multicolumn{10}{|c|}{ Commodity-Carrying Status } & & \\
\hline & \multicolumn{4}{|c|}{ Commodity-Carrying } & \multicolumn{6}{|c|}{ Non-Commodity-Carrying } & & \\
\hline & \multicolumn{2}{|c|}{ Shon Haul } & \multicolumn{2}{|c|}{ Long Haul } & \multicolumn{2}{|c|}{ Businese Use } & \multicolumn{2}{|c|}{ Personal Use } & \multicolumn{2}{|c|}{ Not In Use } & \multicolumn{2}{|c|}{ TOTALs } \\
\hline & Trucks & $\begin{array}{c}\text { Annual } \\
\text { VMT }\end{array}$ & Trucks & $\begin{array}{c}\text { Annual } \\
\text { VMT }\end{array}$ & Trucks & $\begin{array}{c}\text { Annual } \\
\text { VMT }\end{array}$ & Trucks & $\begin{array}{c}\text { Annual } \\
\text { VMT }\end{array}$ & Trucks & $\begin{array}{c}\text { Annual } \\
\text { VMTT }\end{array}$ & Truets & $\begin{array}{c}\text { Annual } \\
\text { VMI }\end{array}$ \\
\hline \multirow[t]{2}{*}{$1987(\mathrm{a})$} & 558 & 13,771 & 100 & 6,516 & 574 & 11,892 & 2,583 & 25,327 & 0 & $\mathbf{0}$ & 3815 & 57,507 \\
\hline & (7.6\%) & $(14.0 \%)$ & $(13.0 \%)$ & $(15.8 \%)$ & $(9.0 \%)$ & $(17 . c \%)$ & (9.5\%) & $(10.9 \%)$ & $(0.0 \%)$ & $(0.0 \%)$ & (9.1\%) & $(131 \%)$ \\
\hline \multirow[t]{2}{*}{1986} & 788 & 14,640 & 101 & 6,976 & 1,098 & 14,941 & 3,614 & 51,357 & $\mathbf{0}$ & $\mathbf{0}$ & 5,601 & 87,914 \\
\hline & $(10.7 \%)$ & $(14.9 \%)$ & $(13.2 \%)$ & (16.9\%) & $(17.2 \%)$ & $(222 \%)$ & $(13.3 \%)$ & $(221 \%)$ & $(0.0 \%)$ & $(0.0 \%)$ & $(13+1 \%)$ & $(200 \%)$ \\
\hline \multirow[t]{2}{*}{1985} & 660 & 13,446 & 103 & 6,790 & 686 & 7,122 & 2,713 & 24,740 & 0 & $\mathbf{0}$ & 4,163 & 52,098 \\
\hline & $(9.0 \%)$ & $(13.7 \%)$ & $(13.5 \%)$ & $(164 \%)$ & $(10.7 \%)$ & $(10.6 \%)$ & $(10.0 \%)$ & $(10.6 \%)$ & $(0.0 \%)$ & $(0.0 \%)$ & (1aox) & $(11.8 \%)$ \\
\hline \multirow[t]{2}{*}{1984} & 496 & 8,085 & 107 & 6,434 & 584 & 6,633 & 2,479 & 25,881 & 0 & $\mathbf{0}$ & 3,667 & 47,003 \\
\hline & $(0.7 \%)$ & (8.2\%) & $(14.0 \%)$ & (15.6\%) & (9.1\%) & $(9.8 \%)$ & (9.1\%) & $(11.1 \%)$ & $(0.0 \%)$ & $(0.0 \%)$ & $(28 \%)$ & $(107 x)$ \\
\hline \multirow[t]{2}{*}{1983} & 344 & 4,092 & 39 & 1,944 & 388 & 3,467 & 2,437 & 24,230 & 2 & 15 & 3,210 & 33,749 \\
\hline & $(4.7 \%)$ & $(4.2 \%)$ & $(5.1 \%)$ & $(4.7 \%)$ & $(0.1 \%)$ & $(5.1 \%)$ & $(9.0 \%)$ & $(10.4 \%)$ & $(1.9 \%)$ & (3.7\%) & $(7.7 x)$ & $(7.7 \%)$ \\
\hline \multirow[t]{2}{*}{1982} & 487 & 7,199 & 29 & 1,463 & 423 & 3,277 & 1,472 & 10,637 & $\mathbf{0}$ & $\mathbf{0}$ & 2,411 & 22,575 \\
\hline & $(6.6 \%)$ & (7.3\%) & $(3.8 \%)$ & (3.5\%) & $(6.6 \%)$ & $(4.9 \%)$ & $(5.4 \%)$ & $(4.6 \%)$ & $(0.0 \%)$ & $(0.0 \%)$ & (5.85) & $(5.1 \%)$ \\
\hline \multirow[t]{2}{*}{1981} & 296 & 3,852 & 38 & 2,065 & 357 & 2,121 & 1,114 & 8,939 & 2 & 1 & 1800 & 16,978 \\
\hline & $(4.0 \%)$ & $(3.9 \%)$ & $(4.9 \%)$ & $(5.0 \%)$ & $(5.6 \%)$ & (3.1\%) & $(4.1 \%)$ & (3.8\%) & $(20 \%)$ & $(0.3 \%)$ & $(43 \times 3)$ & (3.9\%) \\
\hline \multirow[t]{2}{*}{1980} & 330 & 3,913 & 45 & 1,965 & 132 & 1,009 & 706 & 5,538 & 3 & 10 & 1,217 & 12,435 \\
\hline & $(4.5 \%)$ & $(4.0 \%)$ & $(5.9 \%)$ & (4.8\%) & $(2.1 \%)$ & (1.5\%) & $(26 \%)$ & $(24 \%)$ & $(29 \%)$ & $(24 \%)$ & $(29 \%)$ & $(28 \%)$ \\
\hline \multirow[t]{2}{*}{1979} & 624 & 6,638 & 51 & 2,091 & 506 & 4,201 & 2,017 & 9,437 & 7 & 35 & 32005 & 20,403 \\
\hline & (8.5\%) & $(6.7 \%)$ & $(6.6 \%)$ & (5.1\%) & $(7.9 \%)$ & $(6.2 \%)$ & $(7.4 \%)$ & $(4.1 \%)$ & $(0.0 \%)$ & (2.6\%) & $(7.7 \times 5)$ & (s.1\%) \\
\hline \multirow[t]{2}{*}{1978} & 469 & 5,012 & 44 & 1,539 & 192 & 2,425 & 1,748 & 13,030 & 22 & 153 & 2,475 & 22,159 \\
\hline & $(0.4 \%)$ & (5.1\%) & $(5.7 \%)$ & (3.7\%) & $(3.0 \%)$ & (3.6\%) & $(0,4 \%)$ & $(5.6 \%)$ & (18.3\%) & (37.3\%) & (59x) & $(50 \%)$ \\
\hline \multirow[t]{2}{*}{ Pre-1978 } & 2,310 & 17,670 & 108 & 3,507 & 1,454 & 10,297 & 6,240 & 33,617 & 82 & 195 & 10,194 & 65,285 \\
\hline & $(31.4 \%)$ & (18.0\%) & $(14.2 \%)$ & (8.5\%) & $(22.7 \%)$ & (15.3\%) & $(23.0 \%)$ & $(14.4 \%)$ & $(68.8 \%)$ & (47.6\%) & $(2 x+3)$ & $(148 \%)$ \\
\hline \multirow[t]{2}{*}{ Unknown } & 3 & 37 & 0 & 0 & b & b & b & b & 0 & 0 & 3 & 37 \\
\hline & (b) & (b) & $(0.0 \%)$ & $(0.0 \%)$ & (b) & (b) & (b) & (b) & $(0.0 \%)$ & $(0.0 \%)$ & (b) & (b) \\
\hline \multirow[t]{2}{*}{ TOTALS } & 7,365 & 98,354 & 766 & 41,292 & 6,393 & 67,385 & 27,125 & 232,733 & 119 & 410 & 41,768 & 40,174 \\
\hline & $(1000 \%)$ & $(1000 \%)$ & $(1000 x)$ & $(10 a 0 \%)$ & (1000x) & $(1020 x)$ & $(1000 x)$ & $(1000 \%)$ & $(1000 \%)$ & $(1000 \times)$ & $(1000 ; 5)$ & $(1000 \times 5)$ \\
\hline
\end{tabular}

(a) Includes some 1988 models.

(b) Quantity rounded to zero. 
Table 23

Number of Four Year and Older Trucks and Associated Annual Vehicle Miles Traveled (VMT) by Truck Model Year and Truck Type: 1990 NTACS

(Trucks in Thousands/Annual VMT in Millions)

\begin{tabular}{|c|c|c|c|c|c|c|c|c|c|c|c|c|}
\hline \multirow[b]{3}{*}{$\begin{array}{l}\text { Truck } \\
\text { Model } \\
\text { Year } \\
\end{array}$} & \multicolumn{10}{|c|}{ Truck Type } & \multirow{2}{*}{\multicolumn{2}{|c|}{ TOTALS }} \\
\hline & \multicolumn{2}{|c|}{ Pickup } & \multicolumn{2}{|c|}{ Utility } & \multicolumn{2}{|c|}{ Small } & \multicolumn{2}{|c|}{ Large } & \multicolumn{2}{|c|}{ Truck-Tractor } & & \\
\hline & Trucks & $\begin{array}{c}\text { Annual } \\
\text { VMT }\end{array}$ & Trucks & $\begin{array}{c}\text { Annual } \\
\text { VMT }\end{array}$ & Trucks & $\begin{array}{c}\text { Annual } \\
\text { VMT }\end{array}$ & Trucks & $\begin{array}{c}\text { Annual } \\
\text { VMT } \\
\end{array}$ & Trucks & $\begin{array}{c}\text { Annual } \\
\text { VMT }\end{array}$ & Tructs & $\begin{array}{c}\text { Annual } \\
\text { VMT }\end{array}$ \\
\hline \multirow[t]{2}{*}{ 1987(a) } & 2,168 & 26,767 & 1,362 & 18,396 & 127 & 2,190 & 76 & 3,077 & 82 & 7,076 & 3815 & 57,501 \\
\hline & $(88 \%)$ & $(12.3 \%)$ & $(11.1 \%)$ & $(14.9 \%)$ & $(4.5 \%)$ & $(8.7 \%)$ & $(7.3 \%)$ & $(14.9 \%)$ & $(7.8 \%)$ & $(13.3 \%)$ & (P.1\%) & $(13.1 \%)$ \\
\hline \multirow[t]{2}{*}{1986} & 2,710 & 42,334 & 2,464 & 31,196 & 230 & 3,281 & 83 & 2,301 & 115 & 8,802 & 5,601 & 87,914 \\
\hline & $(11.0 \%)$ & (19.5\%) & $(20.0 \%)$ & $(25.2 \%)$ & $(8.1 \%)$ & $(13.0 \%)$ & $(7.9 \%)$ & $(11.1 \%)$ & $(10.9 \%)$ & (16.5\%) & $(13+x)$ & $(200 \%)$ \\
\hline \multirow[t]{2}{*}{1985} & 1,941 & 16,460 & 1,870 & 22,549 & 153 & 2,228 & 79 & 2,034 & 120 & 8,827 & 4,163 & 52,058 \\
\hline & (7.9\%) & $(7.6 \%)$ & $(15.2 \%)$ & $(18.2 \%)$ & $(5.4 \%)$ & $(8.8 \%)$ & $(7.6 \%)$ & $(9.9 \%)$ & (11.5\%) & $(160 \%)$ & $(100 x)$ & $(11.8 \%)$ \\
\hline \multirow[t]{2}{*}{1984} & 2,176 & 25,058 & 1,221 & 12,762 & 108 & 1,714 & 75 & 2,008 & 86 & 5,491 & 3,667 & 47,003 \\
\hline & $(8.9 \%)$ & $(11.5 \%)$ & (9.9\%) & (10.3\%) & $(3.8 \%)$ & $(08 \%)$ & $(7.2 \%)$ & $(9.7 \%)$ & $(8.2 \%)$ & $(10.3 \%)$ & (8.8\%) & (1a7\%) \\
\hline \multirow[t]{2}{*}{1983} & 2,331 & 21,977 & 704 & 6,946 & 107 & 1,789 & 35 & 921 & 33 & 2,116 & 3,210 & 33,749 \\
\hline & $(9.5 \%)$ & $(10.1 \%)$ & $(5.7 \%)$ & $(5.6 \%)$ & $(3.7 \%)$ & $(7.1 \%)$ & $(3.3 \%)$ & $(4.5 \%)$ & $(3.2 \%)$ & $(4.0 \%)$ & $(7.7 x)$ & (7.7\%) \\
\hline \multirow[t]{2}{*}{1982} & 1,770 & 13,533 & 423 & 3,705 & 127 & 2,017 & 39 & 862 & 51 & 2,457 & 2,411 & 22,575 \\
\hline & $(7.2 \%)$ & $(0.2 \%)$ & $(3.4 \%)$ & $(3.0 \%)$ & (4.5\%) & $(8.0 \%)$ & $(3.8 \%)$ & $(4.2 \%)$ & $(4.9 \%)$ & $(4.6 \%)$ & $(5.873)$ & $(\Sigma, \%)$ \\
\hline \multirow[t]{2}{*}{1981} & 1,246 & 8,572 & 310 & 2,848 & 114 & 1,005 & 68 & 1,225 & 70 & 3,330 & 1807 & 16,978 \\
\hline & $(5.1 \%)$ & $(3.9 \%)$ & (25\%) & $(2.3 \%)$ & $(4.0 \%)$ & $(4.0 \%)$ & (6.5\%) & $(5.9 \%)$ & $(6.6 \%)$ & (a.3\%) & $(43 \times 5)$ & $(39 \%)$ \\
\hline \multirow[t]{2}{*}{1980} & 761 & 5,558 & 205 & 1,597 & 103 & 1,129 & 68 & 1,077 & 79 & 3,073 & 1,247 & 12,135 \\
\hline & $(3.1 \%)$ & $(26 \%)$ & (1.7\%) & $(1.3 \%)$ & $(3.6 \%)$ & $(4.5 \%)$ & $(0.6 \%)$ & $(5.2 \%)$ & (7.5\%) & $(5.8 \%)$ & $(29 \times)$ & $(28 \%)$ \\
\hline \multirow[t]{2}{*}{1979} & 1,813 & 10,890 & 1,006 & 5,091 & 224 & 1,657 & 82 & 1,584 & 80 & 3,181 & 3,200 & 20,403 \\
\hline & $(7.4 \%)$ & $(5.0 \%)$ & $(8.2 \%)$ & $(4.1 \%)$ & $(7.9 \%)$ & $(a .6 \%)$ & $(7.9 \%)$ & $(7.7 \%)$ & (7.6\%) & $(0.0 \%)$ & $(7.7 \%)$ & $(5.1 \%)$ \\
\hline \multirow[t]{2}{*}{1978} & 1,644 & 12,008 & 567 & 5,455 & 138 & 1,093 & 66 & 1,425 & 59 & 2,179 & 2,475 & 22,159 \\
\hline & $(6.7 \%)$ & $(5.5 \%)$ & $(4.6 \%)$ & $(4.4 \%)$ & $(4.9 \%)$ & $(4.3 \%)$ & $(6.4 \%)$ & $(0.9 \%)$ & $(5.6 \%)$ & $(4.1 \%)$ & $(29 x)$ & $(5,0 x)$ \\
\hline \multirow[t]{2}{*}{ Pre-1978 } & 5,978 & 34,265 & 2,158 & 13,071 & 1,415 & 7,184 & 369 & 4,101 & 275 & 6,665 & 19,194 & 65,235 \\
\hline & $(24.4 \%)$ & $(15.8 \%)$ & (17.6\%) & (10.6\%) & $(49.7 \%)$ & $(28.4 \%)$ & (35.3\%) & (19.9\%) & $(26.2 \%)$ & (125\%) & $(24+x)$ & $(148 \%)$ \\
\hline \multirow[t]{2}{*}{ Untrnown } & 0 & 0 & 0 & 0 & b & 1 & 3 & 35 & b & 2 & 3 & 37 \\
\hline & $(0.0 \%)$ & $(0.0 \%)$ & $(0.0 \%)$ & $(0.0 \%)$ & (b) & (b) & (0.3\%) & $(0.2 \%)$ & (b) & (b) & (b) & (b) \\
\hline \multirow[t]{2}{*}{ Totrals } & 24,537 & 217,120 & 12,250 & 123,617 & 2847 & 25,287 & 1,044 & 20,650 & 1,050 & 53,199 & 41,768 & 40,174 \\
\hline & (1000\%) & (10nox) & $(1000 \%)$ & $(1000 \%)$ & $(1000 \%)$ & $(1000 \%)$ & $(1000 \%)$ & $(1000 \%)$ & $(1000 \%)$ & $(1000 \%)$ & $(1000 x)$ & (1000\%) \\
\hline
\end{tabular}

(a) Includes some 1988 models.

(b) Quantity rounded to zero. 


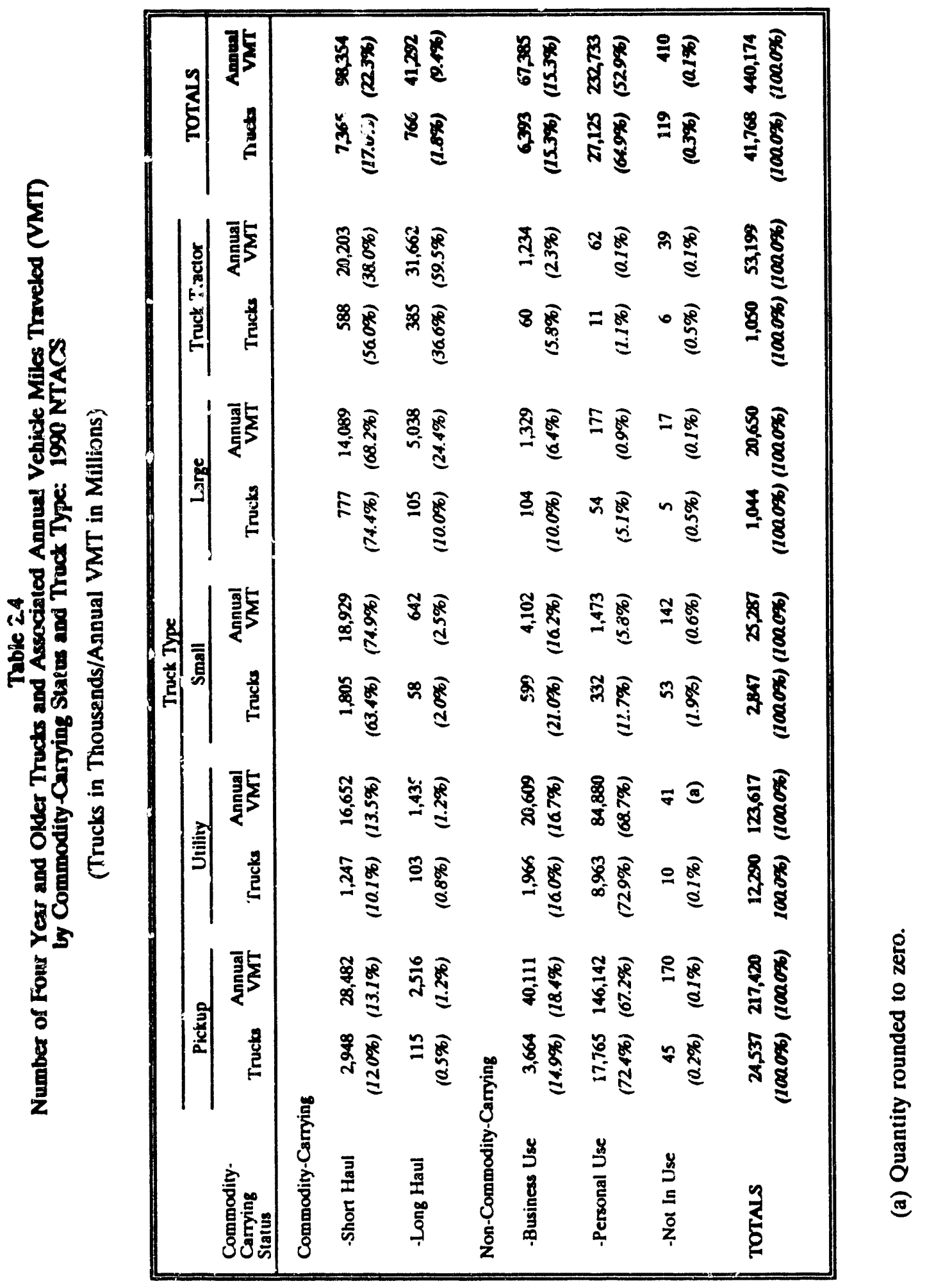




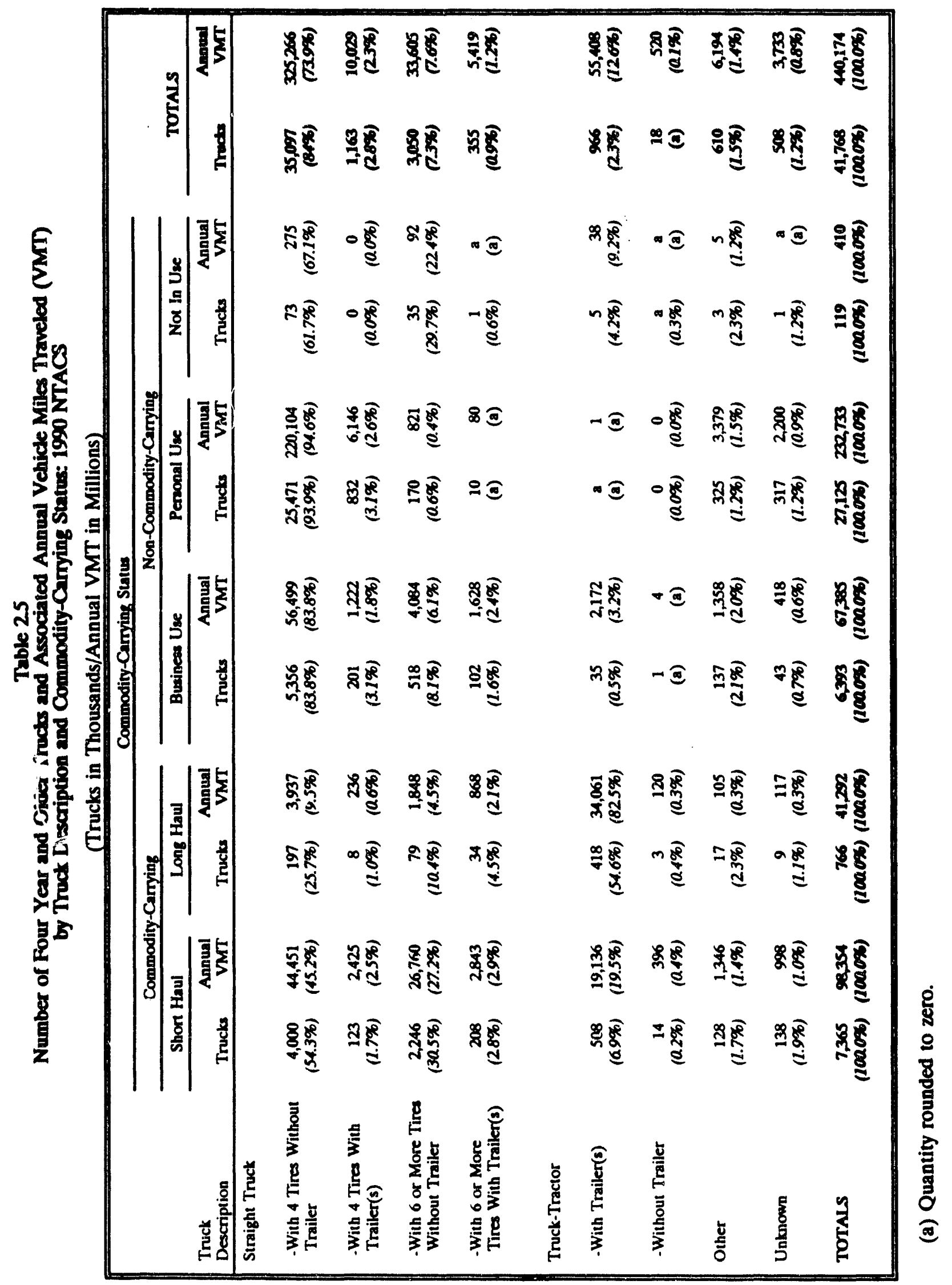




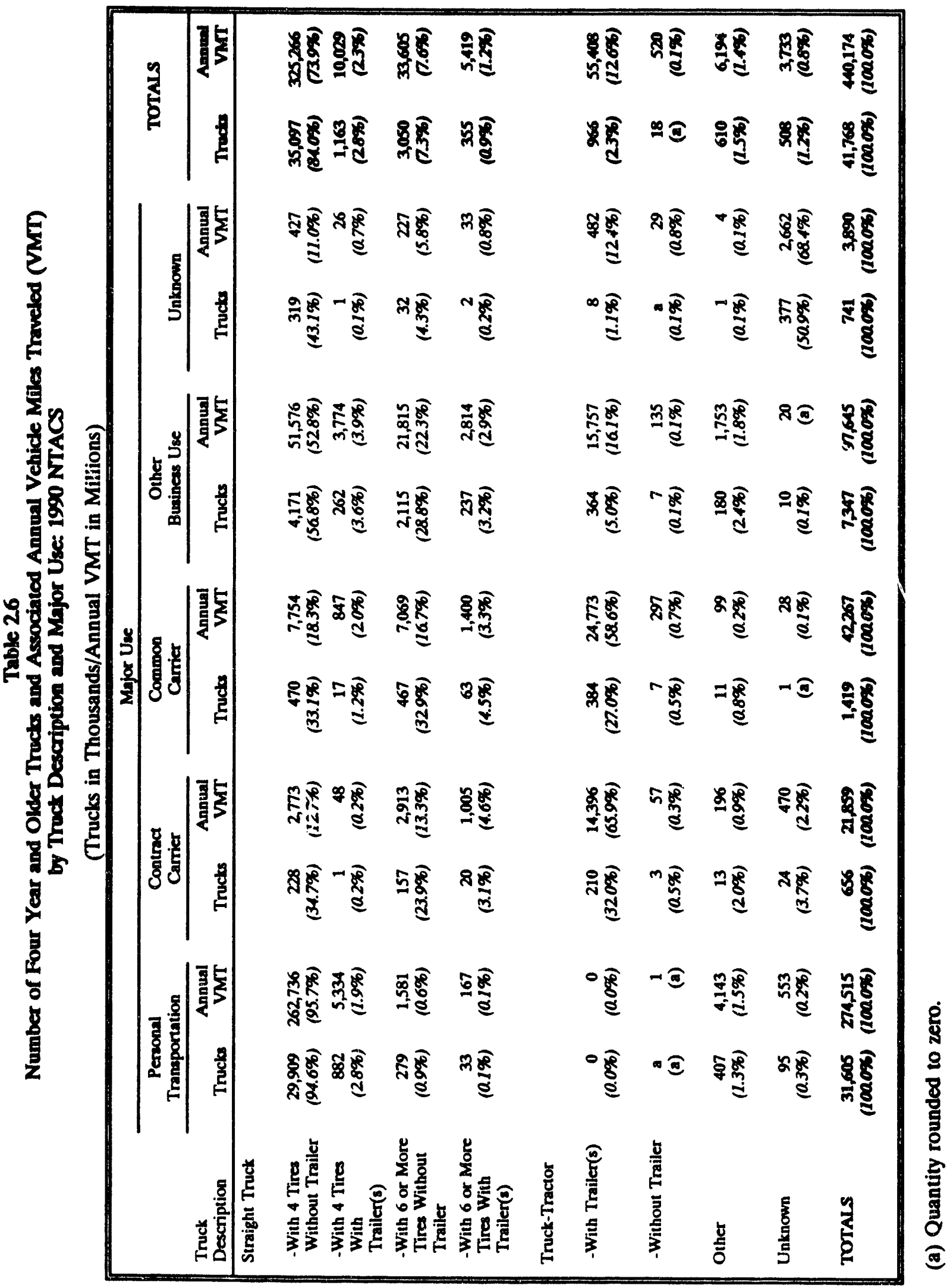


Table 27

Number of Four Year and Older Trucks and Associated Annual Vehicle Miles Traveled (VMT) by Truck Configuration: 1990 NTACS

(Trucks in Thousands/Annual VMT in Millions)

\begin{tabular}{|c|c|c|c|c|}
\hline \multirow{2}{*}{$\begin{array}{l}\text { Truck Configuration } \\
\text { No Trailers Pulled } \ldots \ldots \ldots \ldots \ldots \ldots \ldots \ldots \ldots \ldots \ldots \ldots\end{array}$} & \multicolumn{2}{|c|}{ Trucks } & \multicolumn{2}{|c|}{$\begin{array}{l}\text { Annual } \\
\text { VMT }\end{array}$} \\
\hline & 35,925 & $(86.0 \%)$ & 336,180 & $(76.4 \%)$ \\
\hline \multicolumn{5}{|l|}{ Straight Truck Only, Utility Trailer or Trailer Less Than 20 Feet Long } \\
\hline - One Axle on Trailer Pulled $\ldots \ldots \ldots \ldots \ldots \ldots \ldots \ldots$ & 1,670 & $(4.0 \%)$ & 23,661 & $(5.4 \%)$ \\
\hline - Two Axles on Trailer Pulled $\ldots \ldots \ldots \ldots \ldots \ldots \ldots \ldots$ & 1,370 & $(3.3 \%)$ & 10,938 & (2.5\%) \\
\hline - Three Axles on Trailer Pulled ... & 56 & $(0.1 \%)$ & 1,374 & $(0.3 \%)$ \\
\hline \multicolumn{5}{|l|}{ Straight Truck Only, Full Trailer } \\
\hline - Two Axles on Trailer Pulled ............... & 188 & $(0.5 \%)$ & 2,585 & $(0.6 \%)$ \\
\hline - Three Axles on Trailer Pulled & 8 & (a) & 101 & (a) \\
\hline - Four Axles on Trailer Pulled $\ldots \ldots \ldots \ldots \ldots \ldots \ldots \ldots$ & 1 & (a) & 125 & (a) \\
\hline \multicolumn{5}{|l|}{ Semi-Trailer } \\
\hline - One Axle on Trailer Pulled & 84 & $(0.2 \%)$ & 2,311 & $(0.5 \%)$ \\
\hline - Two Axles on Trailer Pulled & 754 & $(1.8 \%)$ & 42,575 & $(9.7 \%)$ \\
\hline - Three Axles on Trailer Pulled $\ldots \ldots \ldots \ldots \ldots \ldots \ldots \ldots$ & 43 & $(0.1 \%)$ & 2,262 & $(0.5 \%)$ \\
\hline \multicolumn{5}{|l|}{ Two Trailers, One Semi- and One Full } \\
\hline - Total of Three Axles on Two Trailers Pulled & 35 & $(0.1 \%)$ & 3,652 & $(0.8 \%)$ \\
\hline - Total of Four Axles on Two Trailers Pulled $\ldots \ldots \ldots \ldots \ldots \ldots$ & 7 & (a) & 521 & $(0.1 \%)$ \\
\hline \multicolumn{5}{|l|}{ Two or Three Trailers, One Semi- and One Full or One Semi- and Two Full } \\
\hline - Five Axles on Two or Three Trailers Pulled ............. & 6 & (a) & 623 & $(0.1 \%)$ \\
\hline - Six or More Axles on All Trailers Pulled $\ldots \ldots \ldots \ldots \ldots \ldots$ & 6 & (a) & 299 & $(0.1 \%)$ \\
\hline Unkown & 1,614 & $(3.9 \%)$ & 12,966 & $(2.9 \%)$ \\
\hline TOTALS & 41,768 & $(100,0 \%)$ & 440,174 & $(100096)$ \\
\hline
\end{tabular}

(a) Quantity rounded to zero. 
Table 28

Number of Four Year and Older Tructs and Associated Annual Vehicle Miles Traveled (VMT) by Truck Body Type: 1990 NTACS

(Trucks in Thousands/Annual VMT in Millions)

\begin{tabular}{|c|c|c|c|c|}
\hline Truck Body Type & \multicolumn{2}{|c|}{ Trucks } & \multicolumn{2}{|c|}{$\begin{array}{l}\text { Annual } \\
\text { VMT }\end{array}$} \\
\hline $\begin{array}{l}\text { Platform Types - Flatbeds, Stakes, Flatbeds with Added Devices, } \\
\text { and Low Boys (Goosenecks) } \ldots \ldots \ldots \ldots \ldots \ldots \ldots \ldots\end{array}$ & 1,379 & (3.3\%) & 18,213 & $(4.1 \%)$ \\
\hline 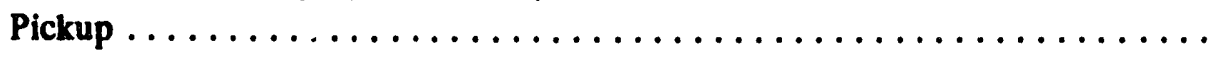 & 23,186 & $(55.5 \%)$ & 213,311 & $(48.5 \%)$ \\
\hline Panel or Compact Van & 2,907 & $(7.0 \%)$ & 35,240 & $(8.0 \%)$ \\
\hline Mini-Van, Utility, Station Wagon (Bronco, Blazer, Jeep, Etc.) . . . . . . . . & 6,191 & $(14.8 \%)$ & 58,447 & (13.3\%) \\
\hline $\begin{array}{l}\text { Van Types - Enclosed Vans, Open Top Vans, Drop Frame Vans, } \\
\text { Refrigerated, Nonrefrigerated, Multistop, and High Cubes } \ldots \ldots\end{array}$ & 2,687 & $(6.4 \%)$ & 53,347 & $(12.1 \%)$ \\
\hline \multicolumn{5}{|l|}{ SPECIALIZED USE TRUCKS } \\
\hline Automobile or Boat Transport $\ldots .$. & 682 & $(1.6 \%)$ & 6,981 & $(1.6 \%)$ \\
\hline 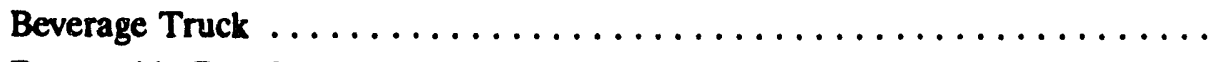 & 127 & $(0.3 \%)$ & 2,425 & $(0.6 \%)$ \\
\hline Removable Dry Container on Trailer Chassis $\ldots \ldots \ldots \ldots \ldots \ldots$ & 23 & $(0.1 \%)$ & 810 & $(0.2 \%)$ \\
\hline Removable Liquid Container on Trailer Chassis ..... & 4 & (a) & 60 & (a) \\
\hline Other Cargo Container Chassis $\ldots \ldots \ldots \ldots \ldots \ldots \ldots \ldots \ldots \ldots \ldots \ldots$ & 165 & $(0.4 \%)$ & 2,540 & $(0.6 \%)$ \\
\hline 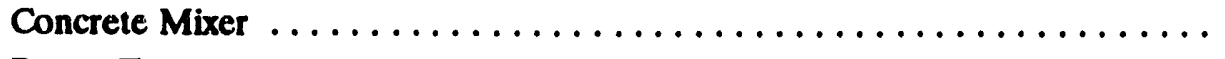 & 85 & $(0.2 \%)$ & 1,066 & $(0.2 \%)$ \\
\hline 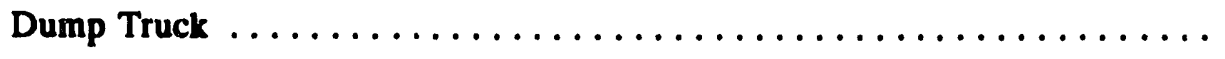 & 699 & $(1.7 \%)$ & 9,279 & (2.1\%) \\
\hline Grain Bodies (Including Hoppers, Grain Boxes) $\ldots \ldots \ldots \ldots \ldots \ldots$ & 355 & $(0.8 \%)$ & 2,707 & $(0.6 \%)$ \\
\hline 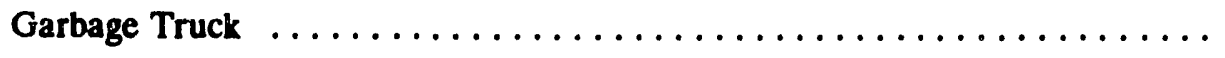 & 56 & $(0.1 \%)$ & 1,149 & $(0.3 \%)$ \\
\hline Livestock Truck, Including Livestock Drop Frame $\ldots \ldots \ldots \ldots \ldots \ldots$ & 198 & (0.5\%) & 3,316 & $(0.8 \%)$ \\
\hline 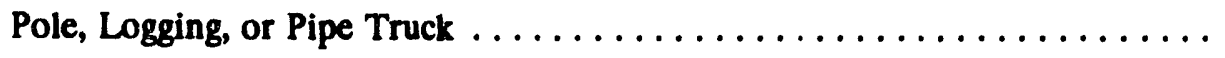 & 73 & $(0.2 \%)$ & 1,961 & $(0.4 \%)$ \\
\hline Tank Truck for Dry Bulk ...... & 43 & $(0.1 \%)$ & 1,923 & $(0.4 \%)$ \\
\hline \multicolumn{5}{|l|}{ Tank Truck for Liquids or Gases } \\
\hline - Nonhazardous Materials .... & 115 & $(0.3 \%)$ & 3,332 & $(0.8 \%)$ \\
\hline 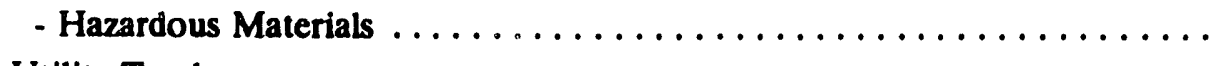 & 131 & $(0.3 \%)$ & 4,235 & $(1.0 \%)$ \\
\hline Utility Truck ........ & 700 & $(1.7 \%)$ & 5,394 & (1.2\%) \\
\hline$\ldots \ldots \ldots$ & 902 & $(2.2 \%)$ & 7,829 & $(1.8 \%)$ \\
\hline$\ldots \ldots \ldots \ldots \ldots \ldots$ & 1,059 & (2.5\%) & 6,611 & (1.5\%) \\
\hline TOTALS & 41,768 & $(100.0 \%)$ & 440,174 & $(100.0 \%)$ \\
\hline
\end{tabular}

(a) Quantity rounded to zero. 
Table 2.9

Number of Four Year and Older Trucks and Associatod Annual Vehicle Miles Traveled (VMT) by Number of States Operated In and Census Region: 1990 NTACS

(Trucks in Thousands/Annual VMT in Millions)

\begin{tabular}{|c|c|c|c|c|c|c|c|c|c|c|}
\hline \multirow{3}{*}{$\begin{array}{l}\text { Number of } \\
\text { States } \\
\text { Operated In }\end{array}$} & \multicolumn{8}{|c|}{ Census Regions } & \multirow{2}{*}{\multicolumn{2}{|c|}{ TOTALs }} \\
\hline & \multicolumn{2}{|c|}{ Northeast } & \multicolumn{2}{|c|}{ Midwest } & \multicolumn{2}{|c|}{ South } & \multicolumn{2}{|c|}{ West } & & \\
\hline & Trucks & $\begin{array}{c}\text { Annual } \\
\text { VMT }\end{array}$ & Trucks & $\begin{array}{c}\text { Annual } \\
\text { VMT }\end{array}$ & Trucks & $\begin{array}{c}\text { Annual } \\
V^{\prime} M^{\prime} T\end{array}$ & Trucks & $\begin{array}{c}\text { Annual } \\
\text { VMT }\end{array}$ & Tructs & $\begin{array}{l}\text { Annual } \\
\text { VMT }\end{array}$ \\
\hline \multirow[t]{2}{*}{ Less Than 3} & 4,171 & 34,656 & 8,140 & 77,854 & 12,354 & 114,079 & 9,283 & 78,071 & 33,947 & 304,660 \\
\hline & (75.9\%) & $(00.5 \%)$ & $(77.6 \%)$ & $(63.7 \%)$ & $(80.8 \%)$ & $(69.1 \%)$ & $(88.5 \%)$ & $(81.7 \%)$ & (81.3\%) & $(69.2 \%)$ \\
\hline \multirow[t]{2}{*}{$3 \cdot 5$} & 856 & 10,304 & 1,611 & 26,593 & 2,213 & 33,957 & 765 & 10,291 & 5,444 & 81,145 \\
\hline & (15.6\%) & $(18.0 \%)$ & $(15.4 \%)$ & $(21.8 \%)$ & $(14.5 \%)$ & $(20.0 \%)$ & $(7.3 \%)$ & $(10.8 \%)$ & $(130 \%)$ & $(184 \%)$ \\
\hline \multirow[t]{2}{*}{6.9} & 308 & 7,510 & 239 & 5,178 & 523 & 10,290 & 304 & 4,633 & 1,375 & 27,612 \\
\hline & $(5.6 \%)$ & $(13.1 \%)$ & $(2.3 \%)$ & $(4.2 \%)$ & $(3.4 \%)$ & $(0.2 \%)$ & $(2.9 \%)$ & $(4.8 \%)$ & $(3.3 \%)$ & (6.3\%) \\
\hline \multirow[t]{2}{*}{$10 \cdot 24$} & 114 & 4,136 & 232 & 6,623 & 157 & 3,972 & 20 & 649 & 523 & 15,380 \\
\hline & $(21 \%)$ & $(7.2 \%)$ & $(22 \%)$ & $(5.4 \%)$ & $(1.0 \%)$ & $(24 \%)$ & $(0.2 \%)$ & $(0.7 \%)$ & $(1.3 \times)$ & $(35 \%)$ \\
\hline \multirow[t]{2}{*}{$25 \cdot 40$} & 3 & 229 & 33 & 4,029 & 17 & 1,940 & 2 & 233 & 55 & 6,431 \\
\hline & (a) & $(0.4 \%)$ & $(0.3 \%)$ & $(3.3 \%)$ & $(0.1 \%)$ & $(1.2 \%)$ & (a) & $(0.2 \%)$ & $(a 18)$ & (1.5\%) \\
\hline \multirow[t]{2}{*}{41 or More } & 1 & 149 & 11 & 1,191 & 5 & 547 & 6 & 990 & 24 & 2877 \\
\hline & (a) & $(0.3 \%)$ & $(0.1 \%)$ & $(1.0 \%)$ & (a) & $(0.3 \%)$ & $(0.1 \%)$ & $(1.0 \%)$ & $(a / x)$ & $(a>\%)$ \\
\hline \multirow[t]{2}{*}{ Unknown } & 40 & 286 & 222 & 754 & 26 & 331 & 111 & 699 & 398 & 2,070 \\
\hline & $(0.7 \%)$ & $(0.5 \%)$ & $(21 \%)$ & $(0.6 \%)$ & $(0.2 \%)$ & $(0.2 \%)$ & $(1.1 \%)$ & $(0.7 \%)$ & $(1.0 \%)$ & $(a .5 \%)$ \\
\hline \multirow[t]{2}{*}{ TOTANS } & 5,493 & 57,270 & 10,489 & 122,202 & 15,295 & 165,116 & 10,491 & 95,566 & 41,768 & 40,174 \\
\hline & (10000x) & (1000\%) & $(1000 \%)$ & $(100.0 \%)$ & $(1000 \%)$ & $(1000 \%)$ & $(100.0 \%)$ & (10000\%) & $(100043)$ & $(1000 \%)$ \\
\hline
\end{tabular}

(a) Quantity rounded to zero. 
Table 2.10

Number of Four Year and Older Trucks and Associated Annual Vehicle Miles Traveled (VMT) by Number of States Operated In and Commodity-Carrying Status: 1990 NTACS

(Trucks in Thousands/Annual VMT in Millions)

\begin{tabular}{|c|c|c|c|c|c|c|c|c|c|c|c|c|}
\hline \multirow[b]{4}{*}{$\begin{array}{l}\text { Number of } \\
\text { States } \\
\text { Operated In }\end{array}$} & \multicolumn{10}{|c|}{ Commodity-Carrying Status } & & \\
\hline & \multicolumn{4}{|c|}{ Commodity-Carrying } & \multicolumn{6}{|c|}{ Non-Commodity-Carrying } & & \\
\hline & \multicolumn{2}{|c|}{ Shon Haul } & \multicolumn{2}{|c|}{ Long Haul } & \multicolumn{2}{|c|}{ Business Use } & \multicolumn{2}{|c|}{ Personal Use } & \multicolumn{2}{|c|}{ Nol In Use } & \multicolumn{2}{|c|}{ TOTALS } \\
\hline & Trucks & $\begin{array}{c}\text { Annual } \\
\text { VMT }\end{array}$ & Trucks & $\begin{array}{c}\text { Annua! } \\
\text { VMT }\end{array}$ & Trucks & $\begin{array}{c}\text { Annual } \\
\mathrm{VMT}\end{array}$ & Trucks & $\begin{array}{c}\text { Annual } \\
\text { VMT }\end{array}$ & Trucks & $\begin{array}{l}\text { Annual } \\
\text { VMT }\end{array}$ & Tructs & $\begin{array}{c}\text { Annual } \\
\text { VMT }\end{array}$ \\
\hline \multirow[t]{2}{*}{ Less Than 3} & 6,744 & 81,997 & 286 & 8,015 & 5,511 & 53,496 & 21,295 & 160,767 & 111 & 384 & 33,947 & 304,660 \\
\hline & $(91.6 \%)$ & $(83.4 \%)$ & $(37.4 \%)$ & $(19.4 \%)$ & $(86.2 \%)$ & $(79.4 \%)$ & $(78.5 \%)$ & $(69.1 \%)$ & $(93.8 \%)$ & $(93.8 \%)$ & (81.3\%) & $(69.2 \%)$ \\
\hline \multirow[t]{2}{*}{$3 \cdot 5$} & 481 & 12,456 & 190 & 9,322 & 682 & 10,454 & 4,092 & 48,912 & $\mathbf{0}$ & 0 & 5,444 & 81,145 \\
\hline & (0.5\%) & $(127 \%)$ & $(24.8 \%)$ & $(226 \%)$ & $(10.7 \%)$ & $(15.5 \%)$ & $(15.1 \%)$ & $(21.0 \%)$ & $(0.0 \%)$ & $(0.0 \%)$ & $(130 \times)$ & $(184 \%)$ \\
\hline \multirow[t]{2}{*}{$6-9$} & 54 & 1,595 & 109 & 6,621 & 95 & 2,060 & 1,117 & 17,335 & 0 & 0 & 1,375 & 27,612 \\
\hline & $(0.7 \%)$ & $(1.6 \%)$ & $(14.2 \%)$ & $(16.0 \%)$ & (1.5\%) & $(3.1 \%)$ & (4.1\%) & (7.4\%) & $(0.0 \%)$ & $(0.0 \%)$ & $(3.3 \%)$ & $(6.3 \%)$ \\
\hline \multirow[t]{2}{*}{$10-24$} & 24 & 1,063 & 99 & 8,212 & 67 & 1,035 & 333 & 5,070 & 0 & 0 & 523 & 15,380 \\
\hline & $(0.3 \%)$ & $(1.1 \%)$ & $(13.0 \%)$ & $(19.9 \%)$ & $(1.1 \%)$ & (1.5\%) & $(1.2 \%)$ & $(22 \%)$ & $(0.0 \%)$ & $(0.0 \%)$ & $(1.3 \%)$ & (35\%) \\
\hline \multirow[t]{2}{*}{$25 \cdot 40$} & 4 & 514 & 51 & 5,917 & 0 & 0 & 0 & 0 & 0 & 0 & S5 & 6,431 \\
\hline & $(0.1 \%)$ & $(0.5 \%)$ & $(6.7 \%)$ & $(14.3 \%)$ & $(0.0 \%)$ & $(0.0 \%)$ & $(0.0 \%)$ & $(0.0 \%)$ & $(0.0 \%)$ & $(0.0 \%)$ & (a.1\%) & (1.5\%) \\
\hline \multirow[t]{2}{*}{41 or More } & $\mathbf{a}$ & 40 & 23 & 2,837 & 0 & 0 & 0 & 0 & 0 & 0 & 24 & 2,877 \\
\hline & (a) & (a) & $(3.0 \%)$ & $(0.9 \%)$ & $(0.0 \%)$ & $(0.0 \%)$ & $(0.0 \%)$ & $(0.0 \%)$ & $(0.0 \%)$ & $(0.0 \%)$ & $(a 1 \%)$ & $(2.7 \%)$ \\
\hline \multirow[t]{2}{*}{ Unknown } & 58 & 689 & 7 & 366 & 38 & 340 & 288 & 648 & 7 & 26 & 398 & 2,070 \\
\hline & $(0.8 \%)$ & $(0.7 \%)$ & $(0.9 \%)$ & $(0.9 \%)$ & $(0.6 \%)$ & $(0.5 \%)$ & $(1.1 \%)$ & $(0.3 \%)$ & $(0.2 \%)$ & $(0.2 \%)$ & $(1.0 \% 3)$ & (as\%) \\
\hline \multirow[t]{2}{*}{ TOTALs } & 7,365 & 98,354 & 766 & 41,292 & 6,393 & 67,385 & 27,125 & 232,733 & 119 & 410 & 41,768 & 40,174 \\
\hline & $(1000 x)$ & $(1000 \times 5)$ & $(1000 x)$ & $(1000 \%)$ & $\left(1000 x_{1}\right)$ & $(100.0 \%)$ & $(1000 \%)$ & $(1000 \%)$ & $(100.0 \%)$ & (1000\%) & (10uox) & (1000\%) \\
\hline
\end{tabular}

(a) Quantity rounded to zero. 
Table 2.11

Number of Four Year and Older Trucks and Associated Annual Vehicle Miles Traveled (VMT) by Number of States Operated In and Truck Type: 1990 NTACS

(Trucks in Thousands/Annual VMT in Millions)

\begin{tabular}{|c|c|c|c|c|c|c|c|c|c|c|c|c|}
\hline \multirow[b]{3}{*}{$\begin{array}{l}\text { Number of } \\
\text { States } \\
\text { Operated In }\end{array}$} & \multicolumn{10}{|c|}{ Truck Type } & \multirow{2}{*}{\multicolumn{2}{|c|}{ TOTALS }} \\
\hline & \multicolumn{2}{|c|}{ Pickup } & \multicolumn{2}{|c|}{ Utility } & \multicolumn{2}{|c|}{ Small } & \multicolumn{2}{|c|}{ Large } & \multicolumn{2}{|c|}{ Truck-Tractor } & & \\
\hline & Trucks & $\begin{array}{c}\text { Annual } \\
\text { VMT }\end{array}$ & Trucks & $\begin{array}{c}\text { Annual } \\
\text { VMT }\end{array}$ & Trucks & $\begin{array}{l}\text { Annual } \\
\text { VMTT }\end{array}$ & Trucks & $\begin{array}{c}\text { Annual } \\
\text { VMT }\end{array}$ & Trucks & $\underset{\text { VMT }}{\text { Annual }}$ & Treats & $\begin{array}{c}\text { Annual } \\
\text { VMII }\end{array}$ \\
\hline Leas Than 3 & $\begin{array}{c}20,910 \\
(85.2 \%)\end{array}$ & $\begin{array}{l}168,120 \\
(77.3 \%)\end{array}$ & $\begin{array}{c}8,808 \\
(71.7 \%)\end{array}$ & $\begin{array}{c}79,196 \\
(64.1 \%)\end{array}$ & $\begin{array}{c}2,673 \\
(93.9 \%)\end{array}$ & $\begin{array}{c}22,898 \\
(90.5 \%)\end{array}$ & $\begin{array}{r}912 \\
(87.4 \%)\end{array}$ & $\begin{array}{c}14,809 \\
(71.7 \%)\end{array}$ & $\begin{array}{r}644 \\
(61.4 \%)\end{array}$ & $\begin{array}{c}19,637 \\
(36,9 \%)\end{array}$ & $\begin{array}{c}33,947 \\
(81.3 \times 3)\end{array}$ & $\begin{array}{c}304,660 \\
(02.2 \% 5)\end{array}$ \\
\hline $3 \cdot 5$ & $\begin{array}{c}2,766 \\
(11.3 \%)\end{array}$ & $\begin{array}{c}35,778 \\
(16.5 \%)\end{array}$ & $\begin{array}{c}2,284 \\
(18.6 \%)\end{array}$ & $\begin{array}{c}28,846 \\
(23.3 \%)\end{array}$ & $\begin{array}{c}131 \\
(4.6 \%)\end{array}$ & $\begin{array}{c}2,125 \\
(8.4 \%)\end{array}$ & $\begin{array}{c}91 \\
(8.7 \%)\end{array}$ & $\begin{array}{r}3,173 \\
(15.4 \%)\end{array}$ & $\begin{array}{r}172 \\
(164 \%)\end{array}$ & $\begin{array}{c}11.224 \\
(21.1 \%)\end{array}$ & $\begin{array}{c}5,44 \\
(130 \times 3)\end{array}$ & $\begin{array}{l}81,145 \\
(184 \%)\end{array}$ \\
\hline $6 \cdot 9$ & $\begin{array}{c}569 \\
(23 \%)\end{array}$ & $\begin{array}{l}12,345 \\
(5.7 \%)\end{array}$ & $\begin{array}{c}715 \\
(5.8 \%)\end{array}$ & $\begin{array}{c}9,223 \\
(7.5 \%)\end{array}$ & $\begin{array}{c}13 \\
(0.4 \%)\end{array}$ & $\begin{array}{c}118 \\
(0.5 \%)\end{array}$ & $\begin{array}{r}18 \\
(1.8 \%)\end{array}$ & $\begin{array}{c}1,397 \\
(0.8 \%)\end{array}$ & $\begin{array}{c}60 \\
(5.8 \%)\end{array}$ & $\begin{array}{c}4,529 \\
(8.5 \%)\end{array}$ & $\begin{array}{c}1,375 \\
(13,35)\end{array}$ & $\begin{array}{l}27,612 \\
(6,3 \%)\end{array}$ \\
\hline $10 \cdot 24$ & $\begin{array}{c}7 \\
(0.0 \%)\end{array}$ & $\begin{array}{c}31 \\
(0.0 \%)\end{array}$ & $\begin{array}{c}407 \\
(3.3 \%)\end{array}$ & $\begin{array}{c}5,933 \\
(4.8 \%)\end{array}$ & $\begin{array}{r}4 \\
(0.1 \%)\end{array}$ & $\begin{array}{r}51 \\
(0.2 \%)\end{array}$ & $\begin{array}{c}11 \\
(1.0 \%)\end{array}$ & $\begin{array}{c}572 \\
(28 \%)\end{array}$ & $\begin{array}{c}94 \\
(9.0 \%)\end{array}$ & $\begin{array}{c}8,794 \\
(16.5 \%)\end{array}$ & $\begin{array}{c}523 \\
(1.3 \times 5)\end{array}$ & $\begin{array}{c}15,380 \\
(3,5 \%)\end{array}$ \\
\hline $25 \cdot 40$ & $\begin{array}{c}0 \\
(0.0 \%)\end{array}$ & $\begin{array}{r}0 \\
(0.0 \%)\end{array}$ & $\begin{array}{c}0 \\
(0.0 \%)\end{array}$ & $\begin{array}{r}0 \\
(0.0 \%)\end{array}$ & $\begin{array}{c}0 \\
(0.0 \%)\end{array}$ & $\begin{array}{r}0 \\
(0.0 \%)\end{array}$ & $\begin{array}{r}5 \\
(0.4 \%)\end{array}$ & $\begin{array}{c}493 \\
(24 \%)\end{array}$ & $\begin{array}{c}51 \\
(4.8 \%)\end{array}$ & $\begin{array}{r}5,939 \\
(11.2 \%)\end{array}$ & $\begin{array}{c}55 \\
\text { (a.15s) }\end{array}$ & $\begin{array}{c}6,431 \\
(1.5 \%)\end{array}$ \\
\hline 41 or More & $\begin{array}{r}0 \\
(a .0 \%)\end{array}$ & $\begin{array}{r}0 \\
(a .0 \%)\end{array}$ & $\begin{array}{c}0 \\
(0.0 \%)\end{array}$ & $\begin{array}{r}0 \\
(0.0 \%)\end{array}$ & $\begin{array}{r}0 \\
(0.0 \%)\end{array}$ & $\begin{array}{c}0 \\
(0.0 \%)\end{array}$ & $\begin{array}{c}1 \\
(0.1 \%)\end{array}$ & $\begin{array}{c}123 \\
(0.6 \%)\end{array}$ & $\begin{array}{r}22 \\
(21 \%)\end{array}$ & $\begin{array}{c}2,755 \\
(5.2 \%)\end{array}$ & $\begin{array}{r}24 \\
(a 1 \%)\end{array}$ & $\begin{array}{c}2877 \\
(a 7 \%)\end{array}$ \\
\hline Unknown & $\begin{array}{c}285 \\
(1.2 \%)\end{array}$ & $\begin{array}{c}1,147 \\
(0.5 \%)\end{array}$ & $\begin{array}{c}77 \\
(0.6 \%)\end{array}$ & $\begin{array}{c}419 \\
(0.3 \%)\end{array}$ & (0.9\%) & $\begin{array}{c}97 \\
(0.4 \%)\end{array}$ & $\begin{array}{r}6 \\
(0.5 \%)\end{array}$ & $\begin{array}{c}85 \\
(0.4 \%)\end{array}$ & $\begin{array}{r}6 \\
(0.6 \%)\end{array}$ & $\begin{array}{c}322 \\
(0.6 \%)\end{array}$ & $\begin{array}{r}3 \times 3 \\
(1.0 \times 3)\end{array}$ & $\begin{array}{c}2,070 \\
(a 5 \%)\end{array}$ \\
\hline TOTALS & $\begin{array}{c}24,537 \\
(1020 ; 3)\end{array}$ & $\begin{array}{c}217,420 \\
(\operatorname{lon} 0,3)\end{array}$ & $\begin{array}{c}12,200 \\
(1020 \% 5)\end{array}$ & $\begin{array}{c}123,617 \\
(1020 \times 5)\end{array}$ & $\begin{array}{c}2847 \\
(10003)\end{array}$ & $\begin{array}{c}25,287 \\
(1000 \%)\end{array}$ & $\begin{array}{c}1,044 \\
(1000 \times 5)\end{array}$ & $\begin{array}{c}20,650 \\
(1020 x)\end{array}$ & $\begin{array}{r}1,050 \\
(1020 \% 5)\end{array}$ & $\begin{array}{c}53,199 \\
(10005)\end{array}$ & $\begin{array}{c}41,768 \\
(10 n c x)\end{array}$ & $\begin{array}{c}40,174 \\
(1000 \times 6)\end{array}$ \\
\hline
\end{tabular}


Table 212

Number of Four Year and Older Tructs and Associnted Annual Vehicle Miles Traveled (VMT) by Number of States Operated In and Major Use: 1990 NTACS

(Trucks in Thousands/Annual VMT in Millions)

\begin{tabular}{|c|c|c|c|c|c|c|c|c|c|c|c|c|}
\hline \multirow[b]{3}{*}{$\begin{array}{l}\text { Number of } \\
\text { States } \\
\text { Opernted In }\end{array}$} & \multicolumn{10}{|c|}{ Major Use } & \multirow{2}{*}{\multicolumn{2}{|c|}{ TOTALS }} \\
\hline & \multicolumn{2}{|c|}{$\begin{array}{c}\text { Personal } \\
\text { Transportation }\end{array}$} & \multicolumn{2}{|c|}{$\begin{array}{l}\text { Contraca } \\
\text { Carrier }\end{array}$} & \multicolumn{2}{|c|}{$\begin{array}{c}\text { Common } \\
\text { Carrier }\end{array}$} & \multicolumn{2}{|c|}{$\begin{array}{l}\text { Other } \\
\text { Busines Use }\end{array}$} & \multicolumn{2}{|c|}{ Unknown } & & \\
\hline & Tructo & $\begin{array}{c}\text { Annual } \\
\text { VMT }\end{array}$ & Trucks & $\begin{array}{c}\text { Annual } \\
\text { VMT }\end{array}$ & Trucks & $\begin{array}{c}\text { Annual } \\
\text { VMT }\end{array}$ & Trucks & $\underset{\text { VMT }}{\text { Annual }}$ & Trucks & $\begin{array}{l}\text { Angual } \\
\text { VMT }\end{array}$ & Truats & Annarel \\
\hline \multirow[t]{2}{*}{ Leas Than 3} & 25.157 & 193,308 & 467 & 11,193 & 1,121 & 21,257 & 6,650 & 76,917 & 553 & 1,985 & 33,947 & 304660 \\
\hline & $(79.6 \%)$ & $(70.4 \%)$ & $(71.2 \%)$ & $(51.2 \%)$ & $(79.0 \%)$ & $(50.3 \%)$ & $(90.5 \%)$ & $(78.8 \%)$ & (74.7\%) & $(51.0 \%)$ & (21.373) & $(a 2 x)$ \\
\hline \multirow[t]{2}{*}{$3 \cdot 5$} & 4,601 & 54,961 & 54 & 3,017 & 160 & 7,236 & 542 & 14,378 & 88 & 1,553 & 5,444 & 81,145 \\
\hline & $(14.6 \%)$ & $(20.0 \%)$ & $(8.2 \%)$ & $(13.8 \%)$ & $(11.3 \%)$ & $(17.1 \%)$ & $(7.1 \%)$ & $(14.7 \%)$ & (11.8\%) & $(39.9 \%)$ & $(130 \times 3)$ & $(12+x)$ \\
\hline \multirow[t]{2}{*}{6.9} & 1,173 & 19,360 & 56 & 1,602 & 36 & 2,912 & 102 & 3,718 & 8 & 19 & 1,375 & 27,612 \\
\hline & $(3.7 \%)$ & $(7.1 \%)$ & $(86 \%)$ & $(7.3 \%)$ & $(26 \%)$ & $(6.9 \%)$ & $(1.4 \%)$ & $(3.8 \%)$ & $(1.0 \%)$ & (a.s\%) & $(323 \times 3)$ & (as3) \\
\hline \multirow[t]{2}{*}{$10 \cdot 24$} & 408 & 5,913 & 37 & 3,444 & 42 & 3,973 & 34 & 1,986 & 3 & 65 & 523 & 15,300 \\
\hline & (1.j\%) & $(22 \%)$ & $(5.6 \%)$ & $(15.8 \%)$ & $(3.0 \%)$ & $(9.4 \%)$ & (a.5\%) & $(20 \%)$ & $(0.4 \%)$ & $(1.7 \%)$ & $(235)$ & (3s) \\
\hline \multirow[t]{2}{*}{$25 \cdot 40$} & 0 & 0 & 11 & 1,488 & $4 l$ & 4,652 & 2 & 268 & $\mathbf{a}$ & 24 & 55 & 6,031 \\
\hline & $(a 0 \%)$ & $(0.0 \%)$ & $(1.7 \%)$ & $(6.8 \%)$ & $(29 \%)$ & $(11.0 \%)$ & (a) & (a.3\%) & (a) & $(a .6 \%)$ & (a13) & $(25 x)$ \\
\hline \multirow[t]{2}{*}{41 or More } & 0 & 0 & $s$ & 570 & 16 & 2,118 & 2 & 174 & $\mathbf{a}$ & 16 & 24 & 2877 \\
\hline & $(0.0 \%)$ & $(0.0 \%)$ & $(0.8 \%)$ & $(26 \%)$ & $(1.2 \%)$ & $(5.0 \%)$ & (a) & $(a .2 \%)$ & (a) & $(0.4 \%)$ & $(a .83)$ & $(a \times x)$ \\
\hline \multirow[t]{2}{*}{ Unknown } & 266 & 973 & 26 & 545 & 2 & 119 & 15 & 204 & 89 & 228 & 303 & 2010 \\
\hline & $(0.8 \%)$ & $(a, 4 \%)$ & $(3.9 \%)$ & (25\%) & $(0.1 \%)$ & (a.3\%) & $(0.2 \%)$ & $(0.2 \%)$ & $(120 \%)$ & $(5.9 \%)$ & $(2.03)$ & (as\%) \\
\hline \multirow[t]{2}{*}{ TOTALS } & $31, \operatorname{ses}$ & 274,515 & 655 & 21.859 & 1,419 & 12207 & 7,317 & 91,645 & 741 & 3800 & $41,7 \notin 8$ & 40,174 \\
\hline & $(1000 x)$ & $(1000 x)$ & $(1000 \times)$ & (10acas) & $(1000 x)$ & $(100 \times x)$ & $(1000 \times)$ & $(1020 x)$ & $(1000 x)$ & $(1000 \times)$ & (10uens) & (1000) \\
\hline
\end{tabular}

(a) Quantity rounded to zero. 
Table 213

Number of Four Year and Older Truclas and Associated Annual Vehicle Miles Traveled (VMT) by Number of Woels Operatod and Commodity-Carrying Status: 1990 NTACS

(Tructs in Thousanda/Annual VMT in Millions)

\begin{tabular}{|c|c|c|c|c|c|c|c|c|c|c|c|c|}
\hline \multirow[b]{4}{*}{$\begin{array}{l}\text { Number } \\
\text { or Weets } \\
\text { Opernied }\end{array}$} & \multicolumn{10}{|c|}{ Commodity-Carrying Status } & \multirow{3}{*}{\multicolumn{2}{|c|}{ TOTALs }} \\
\hline & \multicolumn{4}{|c|}{ Commodity-Carrying } & \multicolumn{6}{|c|}{ Non-Commodity-Carrying } & & \\
\hline & \multicolumn{2}{|c|}{ Shon Haul } & \multicolumn{2}{|c|}{ Lons Haul } & \multicolumn{2}{|c|}{ Busines Use } & \multicolumn{2}{|c|}{ Pernomal Use } & \multicolumn{2}{|c|}{ Not In Use } & & \\
\hline & Trucks & $\begin{array}{l}\text { Angual } \\
\text { VimT }\end{array}$ & Trucks & $\begin{array}{l}\text { Angual } \\
\text { VMMT }\end{array}$ & Trucks & $\begin{array}{l}\text { Annual } \\
\text { VMTT }\end{array}$ & Trucka & $\begin{array}{l}\text { Annual } \\
\text { VMTT }\end{array}$ & Trucks & $\begin{array}{c}\text { Annual } \\
\text { VMuT }\end{array}$ & Trects & Angual \\
\hline 0 & $\begin{array}{c}26 \\
(a, 4 \%)\end{array}$ & (a) & $\begin{array}{c}2 \\
(a .3 \%)\end{array}$ & (a) & $(0.1 \%)$ & (a) & $\begin{array}{c}133 \\
(a .5 \%)\end{array}$ & (a) & $\begin{array}{c}0 \\
(0.0 \%)\end{array}$ & $\begin{array}{c}0 \\
(0.0 \%)\end{array}$ & $\begin{array}{c}165 \\
(a+x)\end{array}$ & $\begin{array}{l}a \\
\text { (a) }\end{array}$ \\
\hline $1 \cdot 4$ & $\begin{array}{c}358 \\
(4.9 \%)\end{array}$ & $\begin{array}{c}130 \\
(a .1 \%)\end{array}$ & $\begin{array}{c}9 \\
(1.2 \%)\end{array}$ & $\begin{array}{l}11 \\
(a)\end{array}$ & $\begin{array}{c}188 \\
(29 \%)\end{array}$ & $\begin{array}{c}72 \\
(a .1 \%)\end{array}$ & $\begin{array}{c}1.306 \\
(4.8 \%)\end{array}$ & $\begin{array}{c}470 \\
(a .2 \%)\end{array}$ & $\begin{array}{c}6 \\
(5.1 \%)\end{array}$ & $\begin{array}{c}a \\
\text { (a) }\end{array}$ & $\begin{array}{l}1805 \\
(45 \times 3)\end{array}$ & $\begin{array}{r}682 \\
(a 2 x)\end{array}$ \\
\hline $3 \cdot 8$ & $\begin{array}{c}198 \\
(27 \%)\end{array}$ & $\begin{array}{c}239 \\
(0.2 \%)\end{array}$ & $\begin{array}{c}11 \\
(1.5 \%)\end{array}$ & $\begin{array}{r}60 \\
(a / \%)\end{array}$ & $\begin{array}{c}78 \\
(1.2 \%)\end{array}$ & $\begin{array}{r}43 \\
(Q .1 \%)\end{array}$ & $\begin{array}{c}458 \\
(1.7 \%)\end{array}$ & $\begin{array}{c}235 \\
(a .1 \%)\end{array}$ & $\begin{array}{c}13 \\
(10.8 \%)\end{array}$ & $\begin{array}{c}3 \\
(1.2 \%)\end{array}$ & $\begin{array}{c}758 \\
(18 \times)\end{array}$ & $\begin{array}{r}582 \\
(a .1 \%)\end{array}$ \\
\hline $9 \cdot 12$ & $\begin{array}{c}261 \\
(3.5 \%)\end{array}$ & $\begin{array}{c}653 \\
(0.7 \%)\end{array}$ & $\begin{array}{r}8 \\
(1.0 \%)\end{array}$ & $\begin{array}{r}60 \\
(0.1 \%)\end{array}$ & $\begin{array}{c}220 \\
(3.4 \%)\end{array}$ & $\begin{array}{c}323 \\
(0.5 \%)\end{array}$ & $\begin{array}{c}631 \\
(23 \%)\end{array}$ & $\begin{array}{c}1.805 \\
(0.8 \%)\end{array}$ & $\begin{array}{c}0 \\
(0.0 \%)\end{array}$ & $\begin{array}{c}0 \\
(0.0 \%)\end{array}$ & $\begin{array}{l}1,119 \\
(27 \times 3)\end{array}$ & $\begin{array}{c}2841 \\
(a 6 \%)\end{array}$ \\
\hline $13 \cdot 16$ & $\begin{array}{c}115 \\
(1.6 \%)\end{array}$ & $\begin{array}{c}264 \\
(0.3 \%)\end{array}$ & $\begin{array}{c}15 \\
(20 \%)\end{array}$ & $\begin{array}{c}79 \\
(a .2 \%)\end{array}$ & $\begin{array}{c}47 \\
(0.7 \%)\end{array}$ & $\begin{array}{c}169 \\
(0.3 \%)\end{array}$ & $\begin{array}{c}213 \\
(0.8 \%)\end{array}$ & $\begin{array}{c}1.249 \\
(0.5 \%)\end{array}$ & $\begin{array}{c}13 \\
(10.8 \%)\end{array}$ & $\begin{array}{c}7 \\
(1.7 \%)\end{array}$ & $\begin{array}{c}400 \\
(1.05)\end{array}$ & $\begin{array}{c}1,767 \\
(a+5 x)\end{array}$ \\
\hline $17 \cdot 20$ & $\begin{array}{c}183 \\
(25 \%)\end{array}$ & $\begin{array}{c}604 \\
(0.6 \%)\end{array}$ & $\begin{array}{c}11 \\
(1.4 \%)\end{array}$ & $\begin{array}{c}120 \\
(a .3 \%)\end{array}$ & $\begin{array}{c}66 \\
(1.0 \%)\end{array}$ & $\begin{array}{c}341 \\
(a .5 \%)\end{array}$ & $\begin{array}{c}754 \\
(28 \%)\end{array}$ & $\begin{array}{c}2,164 \\
(0.9 \%)\end{array}$ & $(a, 3 \%)$ & $\begin{array}{c}0 \\
(0.0 \%)\end{array}$ & $\begin{array}{l}1015 \\
(2 \times 3)\end{array}$ & $\begin{array}{c}3200 \\
(a 7 x)\end{array}$ \\
\hline $21 \cdot 24$ & $\begin{array}{r}92 \\
(1.2 \%)\end{array}$ & $\begin{array}{c}411 \\
(a, 4 \%)\end{array}$ & $\begin{array}{r}6 \\
(0.8 \%)\end{array}$ & $\begin{array}{c}104 \\
(a .3 \%)\end{array}$ & $\begin{array}{c}56 \\
(0.9 \%)\end{array}$ & $\begin{array}{c}302 \\
(a, 4 \%)\end{array}$ & $\begin{array}{c}129 \\
(a .5 \%)\end{array}$ & $\begin{array}{c}2,263 \\
(1.0 \%)\end{array}$ & $\begin{array}{c}0 \\
(0.0 \%)\end{array}$ & $\begin{array}{c}0 \\
(0.0 \%)\end{array}$ & $\begin{array}{c}203 \\
(a 7 x)\end{array}$ & $\begin{array}{c}3079 \\
(27 \times)\end{array}$ \\
\hline $25 \cdot 28$ & $\begin{array}{c}163 \\
(22 \%)\end{array}$ & $\begin{array}{c}956 \\
(1.0 \% 6)\end{array}$ & $\begin{array}{c}22 \\
(28 \%)\end{array}$ & $\begin{array}{c}316 \\
(0.8 \%)\end{array}$ & $\begin{array}{c}60 \\
(0.9 \%)\end{array}$ & $\begin{array}{c}205 \\
(0.3 \%)\end{array}$ & $\begin{array}{c}828 \\
(3.1 \%)\end{array}$ & $\begin{array}{c}2.543 \\
(1.1 \%)\end{array}$ & $\begin{array}{c}1 \\
(0.5 \%)\end{array}$ & $\begin{array}{c}9 \\
(22 \%)\end{array}$ & $\begin{array}{l}1074 \\
(20 \times 3)\end{array}$ & $\begin{array}{l}4000 \\
(a 9 \times)\end{array}$ \\
\hline $29 \cdot 32$ & $\begin{array}{c}200 \\
(27 \%)\end{array}$ & $\begin{array}{c}1,484 \\
(1.5 \%)\end{array}$ & $\begin{array}{r}16 \\
(21 \%)\end{array}$ & $\begin{array}{c}543 \\
(1.3 \%)\end{array}$ & $\begin{array}{c}217 \\
(3.4 \%)\end{array}$ & $\begin{array}{c}1,044 \\
(1.5 \%)\end{array}$ & $\begin{array}{c}359 \\
(1.3 \%)\end{array}$ & $\begin{array}{c}2.647 \\
(1.1 \%)\end{array}$ & $\begin{array}{r}2 \\
(1.5 \%)\end{array}$ & $\begin{array}{c}14 \\
(3.3 \%)\end{array}$ & $\begin{array}{c}7 \times 4 \\
(25 \times 3)\end{array}$ & $\begin{array}{c}5,731 \\
(1,373)\end{array}$ \\
\hline $33 \cdot 36$ & $\begin{array}{c}164 \\
(22 \%)\end{array}$ & $\begin{array}{c}1.272 \\
(1.3 \%)\end{array}$ & $\begin{array}{c}20 \\
(26 \%)\end{array}$ & $\begin{array}{c}403 \\
(1.0 \%)\end{array}$ & $\begin{array}{c}251 \\
(3.9 \%)\end{array}$ & $\begin{array}{c}1,529 \\
(23 \%)\end{array}$ & $\begin{array}{c}76 \\
(a .3 \%)\end{array}$ & $\begin{array}{c}360 \\
(a .2 \%)\end{array}$ & $\begin{array}{r}10 \\
(2.1 \%)\end{array}$ & $\begin{array}{c}17 \\
(4.2 \%)\end{array}$ & $\begin{array}{c}524 \\
(2,25)\end{array}$ & $\begin{array}{c}3501 \\
(a \times x)\end{array}$ \\
\hline $37 \cdot 40$ & $\begin{array}{c}318 \\
(4.3 \%)\end{array}$ & $\begin{array}{c}3,466 \\
(3.5 \%)\end{array}$ & $\begin{array}{c}56 \\
(7.3 \%)\end{array}$ & $\begin{array}{c}1.457 \\
(3.5 \%)\end{array}$ & $\begin{array}{c}288 \\
(4.5 \%)\end{array}$ & $\begin{array}{c}2,415 \\
(3.6 \%)\end{array}$ & $\begin{array}{c}403 \\
(1.5 \%)\end{array}$ & $\begin{array}{c}2.355 \\
(1.0 \%)\end{array}$ & $(0.3 \%)$ & $\begin{array}{c}5 \\
(1.3 \%)\end{array}$ & $\begin{array}{l}1005 \\
(263)\end{array}$ & $\begin{array}{l}2 \times 87 \\
(22 x)\end{array}$ \\
\hline $41 \cdot 44$ & $\begin{array}{c}123 \\
(1.7 \%)\end{array}$ & $\begin{array}{l}2,526 \\
(26 \%)\end{array}$ & $\begin{array}{c}12 \\
(1.5 \%)\end{array}$ & $\begin{array}{c}630 \\
(1.5 \%)\end{array}$ & $(1.0 \%)$ & $\begin{array}{c}791 \\
(1.2 \%)\end{array}$ & $\begin{array}{c}509 \\
(1.9 \%)\end{array}$ & $\begin{array}{c}4.013 \\
(1.7 \%)\end{array}$ & $\begin{array}{c}1 \\
(1.0 \%)\end{array}$ & $\begin{array}{c}1 \\
(a-1 \%)\end{array}$ & $(2.7 \times 3)$ & $\begin{array}{c}7,551 \\
(1.053)\end{array}$ \\
\hline $45 \cdot 48$ & $\begin{array}{c}348 \\
(4.7 \%)\end{array}$ & $\begin{array}{c}6,051 \\
(0.2 \%)\end{array}$ & $\begin{array}{c}56 \\
(7.3 \%)\end{array}$ & $\begin{array}{c}3,709 \\
(9.0 \%)\end{array}$ & $\begin{array}{c}322 \\
(5.0 \%)\end{array}$ & $\begin{array}{c}4.690 \\
(7.0 \%)\end{array}$ & $\begin{array}{c}1,167 \\
(4.3 \%)\end{array}$ & $\begin{array}{l}15,106 \\
(0.5 \%)\end{array}$ & $\begin{array}{c}10 \\
(23 \%)\end{array}$ & $\begin{array}{r}55 \\
(13.4 \%)\end{array}$ & $\begin{array}{c}1500 \\
(4 \times 5)\end{array}$ & $\begin{array}{l}20,510 \\
(a / x)\end{array}$ \\
\hline $49 \cdot 50$ & $\begin{array}{r}612 \\
(8.3 \%)\end{array}$ & $\begin{array}{c}10.001 \\
(10.2 \%)\end{array}$ & $\begin{array}{c}116 \\
(15.2 \%)\end{array}$ & $\begin{array}{c}7.469 \\
(18.1 \%)\end{array}$ & $\begin{array}{r}731 \\
(11 .+\%)\end{array}$ & $\begin{array}{c}8,083 \\
(120 \%)\end{array}$ & $\begin{array}{c}1.525 \\
(5.6 \%)\end{array}$ & $\begin{array}{l}13,888 \\
(6,0 \%)\end{array}$ & $\begin{array}{r}9 \\
(7.3 \%)\end{array}$ & $\begin{array}{r}52 \\
(128 \%)\end{array}$ & $\begin{array}{l}25.3 \\
(7.2 \times 5)\end{array}$ & $\begin{array}{l}30,453 \\
(2.0 \times 5)\end{array}$ \\
\hline $\mid s 1$. & $\begin{array}{c}120 \\
(1.6 \%)\end{array}$ & $\begin{array}{c}2,672 \\
(27 \%)\end{array}$ & $\begin{array}{c}15 \\
(20 \%)\end{array}$ & $\begin{array}{c}1.448 \\
(3.5 \%)\end{array}$ & $\begin{array}{c}63 \\
(1.0 \%)\end{array}$ & $\begin{array}{c}1,021 \\
(1.5 \%)\end{array}$ & $\begin{array}{r}138 \\
\text { (a.5\%) }\end{array}$ & $\begin{array}{r}2,617 \\
(1.1 \%)\end{array}$ & $\begin{array}{c}0 \\
(0.0 \%)\end{array}$ & $\begin{array}{c}0 \\
(0.0 \%)\end{array}$ & $\begin{array}{c}305 \\
(a c 5 s)\end{array}$ & $\begin{array}{c}7,758 \\
(1.0 \times 3)\end{array}$ \\
\hline $\mid s 2$ & $\begin{array}{c}4.084 \\
(55.5 \%)\end{array}$ & $\begin{array}{c}67,627 \\
(02.8 \%)\end{array}$ & $\begin{array}{r}390 \\
(51.0 \%)\end{array}$ & $\begin{array}{c}24.884 \\
(60.3 \%)\end{array}$ & $\begin{array}{c}3,740 \\
(58.5 \%)\end{array}$ & $\begin{array}{c}46,359 \\
(68.8 \%)\end{array}$ & $\begin{array}{c}18.495 \\
(68.2 \%)\end{array}$ & $\begin{array}{c}181,020 \\
(77.8 \%)\end{array}$ & $\begin{array}{r}54 \\
(45.8 \%)\end{array}$ & $\begin{array}{c}244 \\
(59.5 \%)\end{array}$ & $\begin{array}{c}2 \times, 74 \\
(6,1 \times)\end{array}$ & $\frac{300,133}{(227 x)}$ \\
\hline TOTALS & $\begin{array}{c}7,365 \\
(1000 \times 3)\end{array}$ & $\begin{array}{c}90354 \\
(1000 \times 3)\end{array}$ & $\begin{array}{c}765 \\
(1000 \% 3)\end{array}$ & $\begin{array}{c}41,232 \\
(1000 \times 3)\end{array}$ & $\begin{array}{c}6,3 \times 3 \\
(1000 \times 5)\end{array}$ & $\begin{array}{c}67,385 \\
(1000 \times 5)\end{array}$ & $\begin{array}{c}27,125 \\
(1000 x)\end{array}$ & $\begin{array}{c}232,733 \\
(1000 \times 5)\end{array}$ & $\begin{array}{c}119 \\
(1000 \% 5)\end{array}$ & $\begin{array}{c}410 \\
(1000 x)\end{array}$ & $\begin{array}{c}41,763 \\
(\operatorname{ran} 0 \times s)\end{array}$ & $\begin{array}{l}40,174 \\
\text { (1020\%) }\end{array}$ \\
\hline
\end{tabular}

(a) Quantity rounded to zero. 
Table 2.14

Number of Four Year and Older Trucks and Associated Annual Vehicle Miles Traveled (VMT) by Number of Weeks Operated and Truck Type: 1990 NTACS

(Trucks in Thousands/Annual VMT in Millions)

\begin{tabular}{|c|c|c|c|c|c|c|c|c|c|c|c|c|}
\hline \multirow[b]{3}{*}{$\begin{array}{l}\text { Number } \\
\text { of Weeks } \\
\text { Operated }\end{array}$} & \multicolumn{10}{|c|}{ Truck Type } & \multirow{2}{*}{\multicolumn{2}{|c|}{ TOTALS }} \\
\hline & \multicolumn{2}{|c|}{ Pickup } & \multicolumn{2}{|c|}{ Utility } & \multicolumn{2}{|c|}{ Small } & \multicolumn{2}{|c|}{ Large } & \multicolumn{2}{|c|}{ Truck-Tractor } & & \\
\hline & Trucks & $\begin{array}{c}\text { Annual } \\
\text { VMT }\end{array}$ & Trucks & $\begin{array}{c}\text { Annual } \\
\text { VMTT }\end{array}$ & Truckse & $\begin{array}{c}\text { Annual } \\
\text { VMT }\end{array}$ & Trucks & $\begin{array}{c}\text { Annual } \\
\text { VMT }\end{array}$ & Trucks & $\begin{array}{l}\text { Annual } \\
\text { VMTT }\end{array}$ & Truats & $\begin{array}{l}\text { Annual } \\
\text { VMrI }\end{array}$ \\
\hline \multirow[t]{2}{*}{0} & 65 & $\mathbf{a}$ & 82 & $\mathbf{a}$ & 8 & $\mathbf{a}$ & 8 & $\mathbf{a}$ & 2 & $\mathbf{a}$ & 165 & $\mathbf{a}$ \\
\hline & $(0.3 \%)$ & (a) & $(0.7 \%)$ & (a) & $(0.3 \%)$ & (a) & $(0.8 \%)$ & (a) & $(0.2 \%)$ & (a) & $(a+x)$ & (a) \\
\hline \multirow[t]{2}{*}{$1-4$} & 1,223 & 337 & 328 & 201 & 231 & 93 & 48 & 20 & 17 & 31 & 1866 & 682 \\
\hline & $(5.0 \%)$ & $(0.2 \%)$ & $(27 \%)$ & $(0.2 \%)$ & $(8.8 \%)$ & $(0,4 \%)$ & $(4.6 \%)$ & $(0.1 \%)$ & (1.6\%) & $(0.1 \%)$ & $(45 x)$ & $(a x \%)$ \\
\hline \multirow[t]{2}{*}{$5 \cdot 8$} & 433 & 170 & 75 & 131 & 180 & 153 & 41 & 52 & 29 & 77 & 758 & 582 \\
\hline & $(1.8 \%)$ & $(0.1 \%)$ & $(0.6 \%)$ & $(0.1 \%)$ & $(a .3 \%)$ & $(0.6 \%)$ & $(4.0 \%)$ & $(0.2 \%)$ & $(28 \%)$ & $(0.1 \%)$ & $(1.8 \%)$ & $(a 1 \%)$ \\
\hline \multirow[t]{2}{*}{$9 \cdot 12$} & 701 & 1,709 & 178 & 478 & 176 & 320 & 34 & 96 & 31 & 237 & 1,119 & 2841 \\
\hline & $(29 \%)$ & $(0.8 \%)$ & $(1.4 \%)$ & $(0.4 \%)$ & $(0.2 \%)$ & $(1.3 \%)$ & $(3.2 \%)$ & $(0.5 \%)$ & $(3.0 \%)$ & $(0.4 \%)$ & $(27 x)$ & $(0.6 \%)$ \\
\hline \multirow[t]{2}{*}{$13 \cdot 16$} & 137 & 802 & 168 & 624 & 64 & 169 & 25 & 77 & 9 & 95 & 400 & 1,767 \\
\hline & $(0.6 \%)$ & $(0.4 \%)$ & $(1.4 \%)$ & $(0.5 \%)$ & $(22 \%)$ & $(0.7 \%)$ & $(24 \%)$ & $(0.4 \%)$ & $(0.8 \%)$ & $(0.2 \%)$ & $(1.0 \times)$ & $(a+\%)$ \\
\hline \multirow[t]{2}{*}{$17 \cdot 20$} & 756 & 2,099 & 130 & 491 & 81 & 167 & 28 & 256 & 20 & 215 & 1,015 & 3,209 \\
\hline & $(3.1 \%)$ & $(1.0 \%)$ & $(1.1 \%)$ & $(0.4 \%)$ & $(28 \%)$ & $(0.7 \%)$ & $(26 \%)$ & $(1.2 \%)$ & $(1.9 \%)$ & $(0.4 \%)$ & $(2(x)$ & $(a \times \%)$ \\
\hline \multirow[t]{2}{*}{$21 \cdot 24$} & 124 & 2,197 & 91 & 351 & 42 & 246 & 14 & 112 & 12 & 174 & 283 & 3,079 \\
\hline & (a.s\%) & $(1.0 \%)$ & $(0.7 \%)$ & $(0.3 \%)$ & (1.5\%) & $(1.0 \%)$ & $(1.3 \%)$ & $(0.5 \%)$ & $(1.2 \%)$ & $(0.3 \%)$ & $(a 7 x)$ & $(a \times x)$ \\
\hline \multirow[t]{2}{*}{$25 \cdot 28$} & 568 & 1,566 & 337 & 1,294 & 105 & 389 & 35 & 292 & 29 & 488 & 1,074 & 4,020 \\
\hline & $(23 \%)$ & $(a .7 \%)$ & $(27 \%)$ & $(1.0 \%)$ & $(3.7 \%)$ & $(1.5 \%)$ & $(3.4 \%)$ & $(1.4 \%)$ & $(27 \%)$ & $(a .9 \%)$ & $(26 \times)$ & $(a 9 \%)$ \\
\hline \multirow[t]{2}{*}{$29-32$} & 510 & 1,890 & 119 & 2,003 & 85 & 454 & 45 & 660 & 36 & 724 & 794 & 5,731 \\
\hline & $(21 \%)$ & $(0.9 \%)$ & $(1.0 \%)$ & $(1.6 \%)$ & $(3.0 \%)$ & $(1.8 \%)$ & $(4.3 \%)$ & $(3.2 \%)$ & $(3.4 \%)$ & $(1.4 \%)$ & $(1.5 \times 3)$ & $(1.3 \times 5)$ \\
\hline \multirow[t]{2}{*}{$33-36$} & 203 & 798 & 188 & 1,132 & 80 & 558 & 29 & 405 & 21 & 688 & 521 & 3,581 \\
\hline & $(0.8 \%)$ & $(Q+\%)$ & $(1.5 \%)$ & $(0.9 \%)$ & $(28 \%)$ & $(22 \%)$ & $(27 \%)$ & $(20 \%)$ & $(20 \%)$ & (1.3\%) & $(1.2 \times 3)$ & $(a 8 \%)$ \\
\hline \multirow[t]{2}{*}{$37 \cdot 40$} & 601 & 3,713 & 193 & 1,524 & 152 & 1,379 & 63 & 993 & 57 & 2,088 & 1,065 & 9,697 \\
\hline & $(24 \%)$ & $(1.7 \%)$ & $(1.6 \%)$ & $(1.2 \%)$ & $(5.3 \%)$ & $(5.5 \%)$ & $(0.0 \%)$ & $(4.8 \%)$ & $(5.4 \%)$ & $(3.9 \%)$ & $(2 \times 3)$ & $(22 \times 3)$ \\
\hline \multirow[t]{2}{*}{$41 \cdot 44$} & 572 & 4,798 & 61 & 960 & 34 & 885 & 21 & 388 & 20 & 929 & $m$ & 7,561 \\
\hline & (23\%) & $(22 \%)$ & $(0.5 \%)$ & $(0.8 \%)$ & $(1.2 \%)$ & $(3.5 \%)$ & $(20 \%)$ & $(1.9 \%)$ & $(1.9 \%)$ & (1.7\%) & $(1.7 x)$ & (1.8\%) \\
\hline \multirow[t]{2}{*}{$45 \cdot 48$} & 1,229 & 16,950 & 379 & 4,090 & 136 & 1,313 & 69 & 1,597 & 90 & 5,660 & 1,500 & 29,610 \\
\hline & $(5.0 \%)$ & $(7.8 \%)$ & $(3.1 \%)$ & (3.3\%) & $(4.8 \%)$ & $(5.2 \%)$ & $(6.7 \%)$ & $(7.7 \%)$ & (8.5\%) & $(10.6 \%)$ & $(4 \times x)$ & $(a .7 \%)$ \\
\hline \multirow[t]{2}{*}{$49 \cdot 50$} & 1,504 & 16,348 & 1,027 & 8,677 & 235 & 2,547 & 89 & 2,640 & 138 & 9,281 & 2993 & 39,493 \\
\hline & $(6.1 \%)$ & $(7.5 \%)$ & $(8.4 \%)$ & $(7.0 \%)$ & $(8.2 \%)$ & $(10.1 \%)$ & $(8.6 \%)$ & $(128 \%)$ & (13.2\%) & (17.4\%) & $(7.2 \times 3)$ & $(9.0 \%)$ \\
\hline \multirow[t]{2}{*}{51} & 161 & 3,447 & 78 & 467 & 50 & 1,056 & 23 & 886 & 24 & 1,902 & 336 & 7,759 \\
\hline & $(0.7 \%)$ & $(1.6 \%)$ & $(0.6 \%)$ & $(a, 4 \%)$ & $(1.7 \%)$ & $(4.2 \%)$ & $(22 \%)$ & $(4.3 \%)$ & $(23 \%)$ & $(3.6 \%)$ & $(a 8 \times 5)$ & $(1.8 \%)$ \\
\hline \multirow[t]{2}{*}{52} & 15,751 & 160,597 & 8,857 & 101,193 & 1,169 & 15,559 & 473 & 12,174 & 515 & 30,611 & 26,764 & 320,133 \\
\hline & $(64.2 \%)$ & $(73.9 \%)$ & $(72.1 \%)$ & $(81.9 \%)$ & (41.1\%) & $(61.5 \%)$ & $(45.3 \%)$ & $(59.0 \%)$ & $(49.0 \%)$ & $(57.5 \%)$ & $(641 \times)$ & $(727 \%)$ \\
\hline TOTALS & 24,537 & 217,420 & 12,200 & 123,617 & 2847 & 25,287 & 1,044 & 20,650 & 1,050 & 53,199 & 41,768 & 40,174 \\
\hline & $(1000 \%)$ & $(1000 x)$ & $(1000 \%)$ & $(1000 \%)$ & $(1000 \times)$ & $(1000 \times)$ & $\left(1000 x_{0}\right)$ & $(1000 \%)$ & $(1000 \%)$ & $(1000 \%)$ & $(1000 \times 3)$ & $(1000 \% 5)$ \\
\hline
\end{tabular}

(a) Quantity rounded to zero. 
Table 2.15

Number of Four Year and Older Trucks and Assoclated Annual Vehicle Miles Traveled (VMT) by Number of Weels Operated and Major I 'se: 1990 NTACS

(Trucks in Thousands/Annual VMT in Millions)

\begin{tabular}{|c|c|c|c|c|c|c|c|c|c|c|c|c|}
\hline \multirow[b]{3}{*}{$\begin{array}{l}\text { Number of } \\
\text { Weeks } \\
\text { Operated }\end{array}$} & \multicolumn{10}{|c|}{ Major Use } & \multirow{2}{*}{\multicolumn{2}{|c|}{ TOTAls }} \\
\hline & \multicolumn{2}{|c|}{$\begin{array}{c}\text { Personal } \\
\text { Transportation }\end{array}$} & \multicolumn{2}{|c|}{$\begin{array}{l}\text { Contract } \\
\text { Carrier }\end{array}$} & \multicolumn{2}{|c|}{$\begin{array}{l}\text { Common } \\
\text { Carrier }\end{array}$} & \multicolumn{2}{|c|}{$\begin{array}{c}\text { Other } \\
\text { Busineas Use }\end{array}$} & \multicolumn{2}{|c|}{ Unknown } & & \\
\hline & Trucks & $\underset{\text { VMT }}{\text { Annual }}$ & Trucks & $\begin{array}{l}\text { Annual } \\
\text { VMT }\end{array}$ & Trucks & $\begin{array}{c}\text { Annual } \\
\text { VMT }\end{array}$ & Trucks & $\begin{array}{l}\text { Annual } \\
\text { VMT }\end{array}$ & Trucks & $\underset{\text { VMT }}{\text { Annual }}$ & Tructs & $\begin{array}{l}\text { Annual } \\
\text { VMII }\end{array}$ \\
\hline 0 & $\begin{array}{c}68 \\
(0.2 \%)\end{array}$ & (a) & $(0.6 \%)$ & (a) & $\begin{array}{c}0 \\
(0.0 \%)\end{array}$ & $\begin{array}{c}0 \\
(0.0 \%)\end{array}$ & $\begin{array}{r}9 \\
(0.1 \%)\end{array}$ & $\begin{array}{l}a \\
\text { (a) }\end{array}$ & $\begin{array}{r}84 \\
(11.4 \%)\end{array}$ & $\begin{array}{c}a \\
\text { (a) }\end{array}$ & $\begin{array}{c}165 \\
(a(x)\end{array}$ & (a) \\
\hline $1 \cdot 4$ & $\begin{array}{l}1,240 \\
(3.9 \%)\end{array}$ & $\begin{array}{c}448 \\
(0.2 \%)\end{array}$ & $\begin{array}{c}6 \\
(0.9 \%)\end{array}$ & $\begin{array}{c}12 \\
(0.1 \%)\end{array}$ & $\begin{array}{c}48 \\
(3.4 \%)\end{array}$ & $\begin{array}{c}42 \\
(0.1 \%)\end{array}$ & $\begin{array}{c}289 \\
(3.9 \%)\end{array}$ & $\begin{array}{c}110 \\
(0.1 \%)\end{array}$ & $\begin{array}{c}284 \\
(38.3 \%)\end{array}$ & $\begin{array}{c}70 \\
(1.8 \%)\end{array}$ & $\begin{array}{l}1,866 \\
\text { (145\%) }\end{array}$ & $\begin{array}{r}682 \\
(a 2 \%)\end{array}$ \\
\hline $5 \cdot 8$ & $\begin{array}{c}436 \\
(1.4 \%)\end{array}$ & $\begin{array}{c}277 \\
(Q . J \%)\end{array}$ & $\begin{array}{c}5 \\
(0.7 \%)\end{array}$ & $\begin{array}{l}11 \\
\text { (a) }\end{array}$ & $\begin{array}{r}39 \\
(27 \%)\end{array}$ & $\begin{array}{r}49 \\
(0.1 \%)\end{array}$ & $\begin{array}{r}274 \\
(3.7 \%)\end{array}$ & $\begin{array}{r}238 \\
(0.2 \%)\end{array}$ & $\begin{array}{r}4 \\
(0.5 \%)\end{array}$ & $\begin{array}{c}7 \\
(0.2 \%)\end{array}$ & $\begin{array}{c}758 \\
(1.8 \%)\end{array}$ & $\begin{array}{c}582 \\
(a .1 \%)\end{array}$ \\
\hline $9 \cdot 12$ & $\begin{array}{c}822 \\
(26 \%)\end{array}$ & $\begin{array}{c}2,153 \\
(0.8 \%)\end{array}$ & $\begin{array}{c}11 \\
(1.7 \%)\end{array}$ & $\begin{array}{c}65 \\
(0.3 \%)\end{array}$ & $\begin{array}{c}24 \\
(1.7 \%)\end{array}$ & $\begin{array}{c}145 \\
(0.3 \%)\end{array}$ & $\begin{array}{c}258 \\
(3.5 \%)\end{array}$ & $\begin{array}{c}456 \\
(0.5 \%)\end{array}$ & $\begin{array}{r}5 \\
(0.7 \%)\end{array}$ & $\begin{array}{c}23 \\
(0.6 \%)\end{array}$ & $\begin{array}{l}1,119 \\
(27 \times)\end{array}$ & $\begin{array}{c}2841 \\
\left(a 6 x_{0}\right)\end{array}$ \\
\hline $13 \cdot 16$ & $\begin{array}{c}278 \\
(0.9 \%)\end{array}$ & $\begin{array}{c}1,358 \\
(0.5 \%)\end{array}$ & $\begin{array}{r}7 \\
(1.1 \%)\end{array}$ & $\begin{array}{r}36 \\
(0.2 \%)\end{array}$ & $\begin{array}{r}7 \\
(0.5 \%)\end{array}$ & $\begin{array}{r}30 \\
(0.1 \%)\end{array}$ & $\begin{array}{c}108 \\
(1.5 \%)\end{array}$ & $\begin{array}{c}335 \\
(0.3 \%)\end{array}$ & $\begin{array}{c}3 \\
(0.4 \%)\end{array}$ & $\begin{array}{r}8 \\
(0.2 \%)\end{array}$ & $\begin{array}{c}403 \\
(1.0 \%)\end{array}$ & $\begin{array}{c}1,767 \\
(a, 4 \%)\end{array}$ \\
\hline $17 \cdot 20$ & $\begin{array}{c}714 \\
(23 \%)\end{array}$ & $\begin{array}{l}1.424 \\
(0.5 \%)\end{array}$ & $\begin{array}{r}8 \\
(1.1 \%)\end{array}$ & $\begin{array}{r}64 \\
(0.3 \%)\end{array}$ & $\begin{array}{c}86 \\
(a .1 \%)\end{array}$ & $\begin{array}{c}678 \\
(1.6 \%)\end{array}$ & $\begin{array}{c}196 \\
(27 \%)\end{array}$ & $\begin{array}{c}833 \\
(a .9 \%)\end{array}$ & $\begin{array}{c}11 \\
(1.5 \%)\end{array}$ & $\begin{array}{c}229 \\
(5.9 \%)\end{array}$ & $\begin{array}{l}1,015 \\
(2,4)\end{array}$ & $\begin{array}{c}3,2029 \\
(a, 7 \%)\end{array}$ \\
\hline $21 \cdot 24$ & $\begin{array}{c}194 \\
(0.6 \%)\end{array}$ & $\begin{array}{l}2.546 \\
(0.9 \%)\end{array}$ & $\begin{array}{r}3 \\
(a . s \%)\end{array}$ & $\begin{array}{c}51 \\
(0.2 \%)\end{array}$ & $\begin{array}{c}12 \\
(0.8 \%)\end{array}$ & $\begin{array}{c}167 \\
(0.4 \%)\end{array}$ & $\begin{array}{c}73 \\
(1.0 \%)\end{array}$ & $\begin{array}{c}310 \\
(0.3 \%)\end{array}$ & $\begin{array}{c}1 \\
(0.1 \%)\end{array}$ & $\begin{array}{c}5 \\
(0.1 \%)\end{array}$ & $\begin{array}{c}283 \\
(a \times 3)\end{array}$ & $\begin{array}{c}3,079 \\
(a, 7 \%)\end{array}$ \\
\hline $25 \cdot 28$ & $\begin{array}{c}868 \\
(27 \%)\end{array}$ & $\begin{array}{c}2,660 \\
(1.0 \%)\end{array}$ & $\begin{array}{c}13 \\
(20 \%)\end{array}$ & $\begin{array}{c}213 \\
(1.0 \%)\end{array}$ & $\begin{array}{c}41 \\
(29 \%)\end{array}$ & $\begin{array}{c}451 \\
(1.1 \%)\end{array}$ & $\begin{array}{c}146 \\
(20 \%)\end{array}$ & $\begin{array}{c}649 \\
(0.7 \%)\end{array}$ & $\begin{array}{c}5 \\
(0.6 \%)\end{array}$ & $\begin{array}{r}57 \\
(1.5 \%)\end{array}$ & $\begin{array}{l}1,074 \\
(26 \times)\end{array}$ & $\begin{array}{l}4,029 \\
\text { (ases) }\end{array}$ \\
\hline $29 \cdot 32$ & $\begin{array}{c}478 \\
(1.5 \%)\end{array}$ & $\begin{array}{c}3,071 \\
(1.1 \%)\end{array}$ & $\begin{array}{c}17 \\
(26 \%)\end{array}$ & $\begin{array}{c}465 \\
(21 \%)\end{array}$ & $\begin{array}{r}29 \\
(21 \%)\end{array}$ & $\begin{array}{c}442 \\
(1.0 \%)\end{array}$ & $\begin{array}{c}264 \\
(3.6 \%)\end{array}$ & $\begin{array}{c}1,694 \\
(1.7 \%)\end{array}$ & $\begin{array}{c}5 \\
(0.7 \%)\end{array}$ & $\begin{array}{c}58 \\
(1.5 \%)\end{array}$ & $\begin{array}{c}794 \\
(1.9 \times 3)\end{array}$ & $\begin{array}{c}5,731 \\
(1.3 \%)\end{array}$ \\
\hline $33-36$ & $\begin{array}{c}318 \\
(1.0 \%)\end{array}$ & $\begin{array}{c}1,745 \\
(0.6 \%)\end{array}$ & $\begin{array}{c}15 \\
(22 \%)\end{array}$ & $\begin{array}{c}375 \\
(1.7 \%)\end{array}$ & $\begin{array}{c}22 \\
(1.5 \%)\end{array}$ & $\begin{array}{c}423 \\
(1.0 \%)\end{array}$ & $\begin{array}{c}148 \\
(20 \%)\end{array}$ & $\begin{array}{c}998 \\
(1.0 \%)\end{array}$ & $\begin{array}{c}18 \\
(25 \%)\end{array}$ & $\begin{array}{c}40 \\
(1.0 \%)\end{array}$ & $\begin{array}{c}521 \\
(1.2 \times)\end{array}$ & $\begin{array}{l}3581 \\
(2.8 \%)\end{array}$ \\
\hline $37-40$ & $\begin{array}{c}645 \\
(20 \%)\end{array}$ & $\begin{array}{c}3,838 \\
(1.4 \%)\end{array}$ & $\begin{array}{c}35 \\
(5.3 \%)\end{array}$ & $\begin{array}{c}1,059 \\
(4.8 \%)\end{array}$ & $\begin{array}{r}70 \\
(4.9 \%)\end{array}$ & $\begin{array}{c}1.336 \\
(3.2 \%)\end{array}$ & $\begin{array}{c}311 \\
(4.2 \%)\end{array}$ & $\begin{array}{c}3,376 \\
(3.5 \%)\end{array}$ & $\begin{array}{c}5 \\
(0.6 \%)\end{array}$ & $\begin{array}{r}88 \\
(23 \%)\end{array}$ & $\begin{array}{l}1,065 \\
(26 \% 5)\end{array}$ & $\begin{array}{c}9,697 \\
(22 \%)\end{array}$ \\
\hline $41 \cdot 44$ & $\begin{array}{c}605 \\
(1.9 \%)\end{array}$ & $\begin{array}{c}5,535 \\
(20 \%)\end{array}$ & $\begin{array}{c}6 \\
(0.9 \%)\end{array}$ & $\begin{array}{c}301 \\
(1.4 \%)\end{array}$ & $\begin{array}{c}18 \\
(1.3 \%)\end{array}$ & $\begin{array}{c}1,036 \\
(25 \%)\end{array}$ & $\begin{array}{c}76 \\
(1.0 \%)\end{array}$ & $\begin{array}{c}1,036 \\
(1.1 \%)\end{array}$ & $\begin{array}{r}3 \\
(0.4 \%)\end{array}$ & $\begin{array}{c}52 \\
(1.3 \%)\end{array}$ & $\begin{array}{c}708 \\
(1.7 \times 3)\end{array}$ & $\begin{array}{c}7,961 \\
(1.8 \%)\end{array}$ \\
\hline $45 \cdot 48$ & $\begin{array}{c}1,413 \\
(4.5 \%)\end{array}$ & $\begin{array}{l}17,252 \\
(a, 3 \%)\end{array}$ & $\begin{array}{r}49 \\
(7.4 \%)\end{array}$ & $\begin{array}{c}2,589 \\
(11.8 \%)\end{array}$ & $\begin{array}{c}127 \\
(9.0 \%)\end{array}$ & $\begin{array}{c}4,980 \\
(11.8 \%)\end{array}$ & $\begin{array}{c}288 \\
(3.9 \%)\end{array}$ & $\begin{array}{c}4,531 \\
(4.6 \%)\end{array}$ & $\begin{array}{c}27 \\
(3.6 \%)\end{array}$ & $\begin{array}{c}258 \\
(0.6 \%)\end{array}$ & $\begin{array}{l}1,503 \\
(10 \times 6)\end{array}$ & $\begin{array}{l}20,610 \\
(6,7 \%)\end{array}$ \\
\hline $49 \cdot 50$ & $\begin{array}{c}2,070 \\
(0.6 \%)\end{array}$ & $\begin{array}{l}19.657 \\
(7.2 \%)\end{array}$ & $\begin{array}{c}99 \\
(15.0 \%)\end{array}$ & $\begin{array}{c}3,301 \\
(15.1 \%)\end{array}$ & $\begin{array}{c}129 \\
(9.1 \%)\end{array}$ & $\begin{array}{c}5,793 \\
(13.7 \%)\end{array}$ & $\begin{array}{c}678 \\
(9.2 \%)\end{array}$ & $\begin{array}{c}10,606 \\
(10.9 \%)\end{array}$ & $\begin{array}{c}17 \\
(23 \%)\end{array}$ & $\begin{array}{c}137 \\
(3.5 \%)\end{array}$ & $\begin{array}{l}2993 \\
(7.2 \times 3)\end{array}$ & $\begin{array}{l}39,493 \\
(2.0 \% 3)\end{array}$ \\
\hline 51 & $\begin{array}{c}179 \\
(0.6 \%)\end{array}$ & $\begin{array}{c}2,901 \\
(1.1 \%)\end{array}$ & $\begin{array}{c}12 \\
(1.8 \%)\end{array}$ & $\begin{array}{c}987 \\
(4.5 \%)\end{array}$ & $\begin{array}{c}47 \\
(3.3 \%)\end{array}$ & $\begin{array}{c}1.837 \\
(4.3 \%)\end{array}$ & $\begin{array}{r}98 \\
(1.3 \%)\end{array}$ & $\begin{array}{c}2,024 \\
(21 \%)\end{array}$ & $\begin{array}{c}a \\
\text { (a) }\end{array}$ & $\begin{array}{c}10 \\
(0.3 \%)\end{array}$ & $\begin{array}{c}336 \\
(a .8 \%)\end{array}$ & $\begin{array}{c}7,759 \\
(1.8 \%)\end{array}$ \\
\hline 52 & $\begin{array}{c}21,277 \\
(67.3 \%)\end{array}$ & $\begin{array}{l}209,651 \\
(70,4 \%)\end{array}$ & $\begin{array}{c}369 \\
(56.2 \%)\end{array}$ & $\begin{array}{c}12,331 \\
(56+\%)\end{array}$ & $\begin{array}{r}718 \\
(50.6 \%)\end{array}$ & $\begin{array}{c}24,857 \\
(58.8 \%)\end{array}$ & $\begin{array}{c}4,131 \\
(56.2 \%)\end{array}$ & $\begin{array}{c}70,448 \\
(721 \%)\end{array}$ & $\begin{array}{c}269 \\
(36,4 \%)\end{array}$ & $\begin{array}{c}2,846 \\
(73.2 \%)\end{array}$ & $\begin{array}{c}26,764 \\
(641 \%)\end{array}$ & $\begin{array}{l}320,133 \\
(727 \%)\end{array}$ \\
\hline 'TOTALS & $\begin{array}{c}31,605 \\
(100,0 \%)\end{array}$ & $\begin{array}{c}274,515 \\
(1000 \%)\end{array}$ & $\begin{array}{r}656 \\
(100006)\end{array}$ & $\begin{array}{c}21859 \\
(1000 \%)\end{array}$ & $\begin{array}{c}1,419 \\
(1000 \%)\end{array}$ & $\begin{array}{c}42267 \\
(1000 \%)\end{array}$ & $\begin{array}{c}7,347 \\
(1000 \%)\end{array}$ & $\begin{array}{c}97,645 \\
(100,0 \%)\end{array}$ & $\begin{array}{c}741 \\
(1000 \%)\end{array}$ & $\begin{array}{c}3,890 \\
(1000 \%)\end{array}$ & $\begin{array}{c}41,768 \\
(1000 \times)\end{array}$ & $\begin{array}{c}40,174 \\
(1000 \%)\end{array}$ \\
\hline
\end{tabular}

(a) Quantity rounded to zero. 
Table 216

Number of Four Year and Older Trucks

by Annual Miles Traveled and Truck Model Year: 1990 NTACS

(Trucks in Thousands)

\begin{tabular}{|c|c|c|c|c|c|c|c|c|c|c|c|c|c|}
\hline \multirow{2}{*}{$\begin{array}{l}\text { Annual } \\
\text { Miles } \\
\text { Traveled }\end{array}$} & \multicolumn{13}{|c|}{ Truck Model Year } \\
\hline & $\frac{1987(a)}{\text { Iruckas }}$ & Trucks & Trucks & $\frac{1984}{\text { Trucks }}$ & Trucks & Trucks & Trucks & Trucks & Trucks & Trucks & $\begin{array}{l}\text { Pre-1978 } \\
\text { Irucks }\end{array}$ & $\frac{\text { Unknown }}{\text { Irucks }}$ & $\begin{array}{l}\text { Totals } \\
\text { rructs }\end{array}$ \\
\hline $\begin{array}{l}\text { Leas } \\
\text { Than } \\
5,000\end{array}$ & $\begin{array}{r}452 \\
(11.8 \%)\end{array}$ & $\begin{array}{c}788 \\
(14.1 \%)\end{array}$ & $\begin{array}{c}743 \\
(17.8 \%)\end{array}$ & $\begin{array}{c}588 \\
(16.1 \%)\end{array}$ & $\begin{array}{c}418 \\
(13.0 \%)\end{array}$ & $\begin{array}{c}697 \\
(28.9 \%)\end{array}$ & $\begin{array}{c}501 \\
(27.7 \%)\end{array}$ & $\begin{array}{c}367 \\
(30.2 \%)\end{array}$ & $\begin{array}{c}1,176 \\
(36,7 \%)\end{array}$ & $\begin{array}{r}652 \\
(20.4 \%)\end{array}$ & $\begin{array}{c}5,697 \\
(55.9 \%)\end{array}$ & $\underset{(13.2 \%)}{b}$ & $\begin{array}{c}12,079 \\
(289 \%)\end{array}$ \\
\hline $9,5,000-$ & $\begin{array}{c}701 \\
(184 \%)\end{array}$ & $\begin{array}{c}984 \\
(17.6 \%)\end{array}$ & $\begin{array}{r}1,116 \\
(268 \%)\end{array}$ & $\begin{array}{c}977 \\
(26.6 \%)\end{array}$ & $\begin{array}{c}703 \\
(21.9 \%)\end{array}$ & $\begin{array}{r}594 \\
(24.7 \%)\end{array}$ & $\begin{array}{c}500 \\
(27.7 \%)\end{array}$ & $\begin{array}{r}239 \\
(19.7 \%)\end{array}$ & $\begin{array}{c}910 \\
(28.4 \%)\end{array}$ & $\begin{array}{c}860 \\
(34.8 \%)\end{array}$ & $\begin{array}{c}2,204 \\
(21.6 \%)\end{array}$ & $(20 \%)$ & $\begin{array}{r}9,789 \\
(23,4 \%)\end{array}$ \\
\hline $\begin{array}{l}10,000 \\
14,999\end{array}$ & $\begin{array}{c}801 \\
(21.0 \%)\end{array}$ & $\begin{array}{c}1,972 \\
(35.2 \%)\end{array}$ & $\begin{array}{c}990 \\
(23.8 \%)\end{array}$ & $\begin{array}{c}917 \\
(25.0 \%)\end{array}$ & $\begin{array}{c}1,323 \\
(41.2 \%)\end{array}$ & $\begin{array}{c}484 \\
(20.1 \%)\end{array}$ & $\begin{array}{c}493 \\
(27.3 \%)\end{array}$ & $\begin{array}{c}337 \\
(27.7 \%)\end{array}$ & $\begin{array}{c}618 \\
(19.3 \%)\end{array}$ & $\begin{array}{c}681 \\
(27.5 \%)\end{array}$ & $\begin{array}{c}1,630 \\
(160 \%)\end{array}$ & $(81.0 \%)$ & $\begin{array}{c}10,249 \\
(24,5 \%)\end{array}$ \\
\hline $\begin{array}{l}15,000 \\
19,999\end{array}$ & $\begin{array}{r}714 \\
(187 \%)\end{array}$ & $\begin{array}{r}654 \\
(11.7 \%)\end{array}$ & $\begin{array}{c}633 \\
(15.2 \%)\end{array}$ & $\begin{array}{c}394 \\
(10.7 \%)\end{array}$ & $\begin{array}{c}415 \\
(129 \%)\end{array}$ & $\begin{array}{c}127 \\
(5.3 \%)\end{array}$ & $\begin{array}{c}96 \\
(5.3 \%)\end{array}$ & $\begin{array}{c}130 \\
(10.7 \%)\end{array}$ & $\begin{array}{c}225 \\
(7.0 \%)\end{array}$ & $\begin{array}{c}79 \\
(3.2 \%)\end{array}$ & $\begin{array}{c}290 \\
(28 \%)\end{array}$ & $\left(\begin{array}{c}0 \\
(0.0 \%)\end{array}\right.$ & $\begin{array}{c}3.757 \\
(9.0 \%)\end{array}$ \\
\hline $\mid \begin{array}{l}20,000 \\
24,999\end{array}$ & $\begin{array}{c}419 \\
(11.0 \%)\end{array}$ & $\begin{array}{c}700 \\
(125 \%)\end{array}$ & $\begin{array}{c}448 \\
(10.8 \%)\end{array}$ & $\begin{array}{c}383 \\
(10.4 \%)\end{array}$ & $\begin{array}{c}231 \\
(7.2 \%)\end{array}$ & $\begin{array}{c}34 \\
(1.4 \%)\end{array}$ & $\begin{array}{c}20 \\
(1.1 \%)\end{array}$ & $\begin{array}{c}27 \\
(2.2 \%)\end{array}$ & $\begin{array}{c}60 \\
(1.9 \%)\end{array}$ & $\begin{array}{c}40 \\
(1.6 \%)\end{array}$ & $\begin{array}{c}102 \\
(1.0 \%)\end{array}$ & $\begin{array}{c}b \\
(29 \%)\end{array}$ & $\begin{array}{l}2,462 \\
(5,9 \%)\end{array}$ \\
\hline 25,000 & $\begin{array}{c}206 \\
(5.4 \%)\end{array}$ & $\begin{array}{c}249 \\
(4.4 \%)\end{array}$ & $\begin{array}{c}53 \\
(1.3 \%)\end{array}$ & $\begin{array}{c}225 \\
(0.1 \%)\end{array}$ & $\begin{array}{c}36 \\
(1.1 \%)\end{array}$ & $\begin{array}{c}209 \\
(2.7 \%)\end{array}$ & $\begin{array}{c}35 \\
(1.9 \%)\end{array}$ & $\begin{array}{c}45 \\
(3.7 \%)\end{array}$ & $\left(\begin{array}{c}11 \\
(0.3 \%)\end{array}\right.$ & $\begin{array}{c}59 \\
(24 \%)\end{array}$ & $\begin{array}{c}32 \\
(0.3 \%)\end{array}$ & $\begin{array}{c}0 \\
(0.0 \%)\end{array}$ & $\begin{array}{c}1,158 \\
(28 \%)\end{array}$ \\
\hline 39,000 & $\begin{array}{c}280 \\
(7.3 \%)\end{array}$ & $\begin{array}{c}90 \\
(1.6 \%)\end{array}$ & $\begin{array}{c}42 \\
(1.0 \%)\end{array}$ & $\begin{array}{c}82 \\
(22 \%)\end{array}$ & $\begin{array}{c}29 \\
(0.9 \%)\end{array}$ & $\begin{array}{c}45 \\
(1.9 \%)\end{array}$ & $\begin{array}{c}83 \\
(4.6 \%)\end{array}$ & $\begin{array}{c}14 \\
(1.2 \%)\end{array}$ & $\begin{array}{c}12 \\
(0.4 \%)\end{array}$ & $\begin{array}{c}73 \\
(29 \%)\end{array}$ & $\begin{array}{c}69 \\
(0.7 \%)\end{array}$ & $(0.0 \%)$ & $\begin{array}{r}819 \\
(20 \%)\end{array}$ \\
\hline 40,000 & $\begin{array}{c}88 \\
(2.3 \%)\end{array}$ & $\begin{array}{c}43 \\
(0.8 \%)\end{array}$ & $\begin{array}{c}41 \\
(1.0 \%)\end{array}$ & $\begin{array}{c}35 \\
(1.0 \%)\end{array}$ & $\begin{array}{c}9 \\
(0.3 \%)\end{array}$ & $\begin{array}{c}61 \\
(25 \%)\end{array}$ & $\begin{array}{c}40 \\
(22 \%)\end{array}$ & $\begin{array}{c}15 \\
(1.2 \%)\end{array}$ & $\begin{array}{c}21 \\
(0.7 \%)\end{array}$ & $\begin{array}{c}7 \\
(0.3 \%)\end{array}$ & $\begin{array}{c}103 \\
(1.0 \%)\end{array}$ & $(0.0 \%)$ & $\begin{array}{r}463 \\
(1.1 \%)\end{array}$ \\
\hline 50,000 & $\begin{array}{c}92 \\
(24 \%)\end{array}$ & $\begin{array}{c}56 \\
(1.0 \%)\end{array}$ & $\begin{array}{c}38 \\
(0.9 \%)\end{array}$ & $\begin{array}{c}23 \\
(0.6 \%)\end{array}$ & $\begin{array}{c}27 \\
(0.8 \%)\end{array}$ & $\begin{array}{c}135 \\
(5.6 \%)\end{array}$ & $\begin{array}{c}22 \\
(1.2 \%)\end{array}$ & $\begin{array}{c}19 \\
(1.6 \%)\end{array}$ & $\begin{array}{c}21 \\
(0.6 \%)\end{array}$ & $\begin{array}{c}14 \\
(0.6 \%)\end{array}$ & $\begin{array}{c}36 \\
(0.3 \%)\end{array}$ & $\begin{array}{c}0 \\
(0.0 \%)\end{array}$ & $\begin{array}{r}481 \\
(1.2 \%)\end{array}$ \\
\hline $\begin{array}{l}75,000 \\
99,999\end{array}$ & $\begin{array}{c}26 \\
(a .7 \%)\end{array}$ & $\begin{array}{c}23 \\
(0.4 \%)\end{array}$ & $\begin{array}{c}20 \\
(0.5 \%)\end{array}$ & $\begin{array}{c}25 \\
(0.7 \%)\end{array}$ & $(0.2 \%)$ & $\begin{array}{r}9 \\
(0.4 \%)\end{array}$ & $\begin{array}{c}12 \\
(0.7 \%)\end{array}$ & $\begin{array}{r}{ }^{7} \\
(0.6 \%)\end{array}$ & $\begin{array}{c}105 \\
(3.3 \%)\end{array}$ & $\begin{array}{r}8 \\
(0.3 \%)\end{array}$ & $(0.2 \%)$ & $\begin{array}{c}0 \\
(0.0 \%)\end{array}$ & $\begin{array}{r}259 \\
(a 6 \%)\end{array}$ \\
\hline $\begin{array}{l}100,000 \\
124,999\end{array}$ & $\begin{array}{c}20 \\
(0.5 \%)\end{array}$ & $\begin{array}{c}27 \\
(0.5 \%)\end{array}$ & $\begin{array}{c}23 \\
(0.6 \%)\end{array}$ & $\begin{array}{c}14 \\
(0.4 \%)\end{array}$ & $\begin{array}{r}12 \\
(0.4 \%)\end{array}$ & $\begin{array}{c}16 \\
(0.7 \%)\end{array}$ & $\begin{array}{c}6 \\
(0.3 \%)\end{array}$ & $(0.2 \%)$ & $(0.1 \%)$ & $\frac{1}{(0.1 \%)}$ & $(0.1 \%)$ & $\begin{array}{c}0 \\
(0.0 \%)\end{array}$ & (a3\%) \\
\hline $\begin{array}{l}125,000 \\
149,999\end{array}$ & $(0.2 \%)$ & $(a .1 \%)$ & $\frac{7}{(0.2 \%)}$ & $(0.1 \%)$ & $\begin{array}{l}b \\
\text { (b) }\end{array}$ & $\begin{array}{c}b \\
\text { (b) }\end{array}$ & $\begin{array}{l}b \\
\text { (b) }\end{array}$ & $\begin{array}{c}12 \\
(1.0 \%)\end{array}$ & $\begin{array}{c}b \\
\text { (b) }\end{array}$ & $\begin{array}{l}\text { b } \\
\text { (b) }\end{array}$ & $\begin{array}{c}b \\
\text { (b) }\end{array}$ & $(0.0 \%)$ & $\begin{array}{r}37 \\
\text { (a.1\%) }\end{array}$ \\
\hline $\begin{array}{l}150,000 \\
199,999\end{array}$ & $(0.2 \%)$ & $(0.2 \%)$ & $(0.1 \%)$ & $\begin{array}{c}2 \\
\text { (b) }\end{array}$ & (b) & $\begin{array}{c}0 \\
(0.0 \%)\end{array}$ & $\begin{array}{c}0 \\
(0.0 \%)\end{array}$ & $\begin{array}{l}\text { b } \\
\text { (b) }\end{array}$ & $\begin{array}{l}\text { b } \\
\text { (b) }\end{array}$ & $\begin{array}{c}0 \\
(0.0 \%)\end{array}$ & $\begin{array}{l}1 \\
\text { (b) }\end{array}$ & $(0.0 \%)$ & $\stackrel{27}{(a .1 \%)}$ \\
\hline $\begin{array}{l}200,000 \\
\text { and Over }\end{array}$ & $\begin{array}{l}1 \\
\text { (b) }\end{array}$ & $\begin{array}{c}1 \\
\text { (b) }\end{array}$ & $\begin{array}{r}3^{3} \\
(0.1 \%)\end{array}$ & $\begin{array}{c}0 \\
(0.0 \%)\end{array}$ & $\begin{array}{l}\text { b } \\
\text { (b) }\end{array}$ & $\begin{array}{c}1 \\
\text { (b) }\end{array}$ & $\begin{array}{c}b \\
\text { (b) }\end{array}$ & $\begin{array}{r}1 \\
(0.1 \%)\end{array}$ & $\begin{array}{c}42 \\
(1.3 \%)\end{array}$ & $\begin{array}{r}0 \\
(0.0 \%)\end{array}$ & $\stackrel{2}{\text { (b) }}$ & $\begin{array}{c}0 \\
(0.0 \%)\end{array}$ & $\begin{array}{r}51 \\
(0.1 \%)\end{array}$ \\
\hline Totrals & $\begin{array}{c}3815 \\
(1000 \%)\end{array}$ & $\begin{array}{c}5,601 \\
(1000 \%)\end{array}$ & $\begin{array}{c}4,163 \\
(1020 \%)\end{array}$ & $\begin{array}{c}3,667 \\
(100 \% \%)\end{array}$ & $\begin{array}{c}3,210 \\
(1000 \%)\end{array}$ & $\begin{array}{c}2,411 \\
(1000 \%)\end{array}$ & $\begin{array}{c}1,800 \\
(1000 \%)\end{array}$ & $\begin{array}{c}1,217 \\
(1000 \%)\end{array}$ & $\begin{array}{c}3,2005 \\
(100,0 \%)\end{array}$ & $\begin{array}{c}2,475 \\
(1000 \%)\end{array}$ & $\begin{array}{c}10,194 \\
\text { (1000\%) }\end{array}$ & $(1000 \%)$ & $\begin{array}{r}41,768 \\
(100 \% 0 \%)\end{array}$ \\
\hline
\end{tabular}

(a) Includes some 1988 models.

(b) Quantity rounded to zero. 
Table 2.17

Number of Four Year and Older Trucks

by Annual Miles Traveled and Census Region: 1990 NTACS

(Trucks in Thousands)

\begin{tabular}{|c|c|c|c|c|c|}
\hline \multirow[b]{2}{*}{$\begin{array}{l}\text { Annual Miles } \\
\text { Traveled }\end{array}$} & \multicolumn{4}{|c|}{ Census Regions } & \multirow[b]{2}{*}{$\frac{\text { TOTALS }}{\text { Trucks }}$} \\
\hline & $\frac{\text { Northeast }}{\text { Irucks }}$ & $\frac{\text { Midwest }}{\text { Trucks }}$ & $\begin{array}{l}\text { South } \\
\text { Trucks }\end{array}$ & $\frac{\text { West }}{\text { Trucks }}$ & \\
\hline \multirow[t]{2}{*}{ Less than 5,000} & 1,222 & 2,960 & 4,180 & 3,718 & 12,079 \\
\hline & $(22.2 \%)$ & $(28.2 \%)$ & $(27.3 \%)$ & $(35.4 \%)$ & (289\%) \\
\hline \multirow[t]{2}{*}{$5,000-9,999$} & 1,651 & 2,261 & 3,430 & 2,447 & 9,789 \\
\hline & $(30.1 \%)$ & $(21.6 \%)$ & $(22.4 \%)$ & $(23.3 \%)$ & (23.4\%) \\
\hline \multirow[t]{2}{*}{$10,000-14,999$} & 1,389 & 2,678 & 3,635 & 2,547 & 10,249 \\
\hline & $(25.3 \%)$ & $(25.5 \%)$ & $(23.8 \%)$ & $(24.3 \%)$ & (24.5\%) \\
\hline \multirow[t]{2}{*}{$15,000-19,999$} & 627 & 1,015 & 1,483 & 632 & 3,757 \\
\hline & $(11.4 \%)$ & (9.7\%) & $(9.7 \%)$ & $(6.0 \%)$ & $(9.0 \%)$ \\
\hline \multirow[t]{2}{*}{$20,000-24,999$} & 181 & 662 & 1,006 & 613 & 2,462 \\
\hline & $(3.3 \%)$ & $(6.3 \%)$ & $(6.6 \%)$ & $(5.8 \%)$ & $(5.9 \%)$ \\
\hline \multirow[t]{2}{*}{$25,000-29,999$} & 194 & 146 & 688 & 130 & 1,158 \\
\hline & (3.5\%) & $(1.4 \%)$ & $(4.5 \%)$ & $(1.2 \%)$ & $(2.8 \%)$ \\
\hline \multirow[t]{2}{*}{$30,000-39,999$} & 71 & 282 & 342 & 124 & 819 \\
\hline & $(1.3 \%)$ & $(2.7 \%)$ & $(2.2 \%)$ & $(1.2 \%)$ & $(20 \%)$ \\
\hline \multirow[t]{2}{*}{$40,000-49,999$} & 29 & 72 & 174 & 188 & 463 \\
\hline & $(0.5 \%)$ & $(0.7 \%)$ & $(1.1 \%)$ & $(1.8 \%)$ & $(1.1 \%)$ \\
\hline \multirow[t]{2}{*}{$50,000-74,999$} & 41 & 233 & 167 & 40 & 481 \\
\hline & $(0.7 \%)$ & $(2.2 \%)$ & $(1.1 \%)$ & $(0.4 \%)$ & $(1.2 \%)$ \\
\hline \multirow[t]{2}{*}{$75,000-99,999$} & 77 & 61 & 99 & 22 & 259 \\
\hline & $(1.4 \%)$ & $(0.6 \%)$ & $(0.6 \%)$ & $(0.2 \%)$ & $(0.6 \%)$ \\
\hline \multirow[t]{2}{*}{$100,000-124,999$} & 9 & 55 & 56 & 17 & 137 \\
\hline & $(0.2 \%)$ & $(0.5 \%)$ & $(0.4 \%)$ & $(0.2 \%)$ & $(0.3 \%)$ \\
\hline \multirow[t]{2}{*}{$125,000-149,999$} & 1 & 8 & 22 & 6 & 37 \\
\hline & (a) & $(0.1 \%)$ & $(0.1 \%)$ & $(0.1 \%)$ & $(0.1 \%)$ \\
\hline \multirow[t]{2}{*}{$150,000-199,999$} & 1 & 15 & 6 & 5 & 27 \\
\hline & (a) & $(0.1 \%)$ & (a) & $(0.1 \%)$ & (0.1\%) \\
\hline \multirow[t]{2}{*}{200,000 and over } & 1 & 40 & 8 & 1 & 51 \\
\hline & (a) & $(0.4 \%)$ & $(0.1 \%)$ & (a) & $(0.1 \%)$ \\
\hline \multirow[t]{2}{*}{ TOTALS } & 5,493 & 10,489 & 15,295 & 10,491 & 41,768 \\
\hline & $(100.0 \%)$ & $(100.0 \%)$ & $(100.0 \%)$ & $(100.0 \%)$ & $(1000 \%)$ \\
\hline
\end{tabular}

(a) Quantity rounded to zero. 
Table 218

Number of Four Year and Older Trurks

by Annual Miles Traveled and Commodity-Carrying Status: 1990 NTACS

(Trucks in Thousands)

\begin{tabular}{|c|c|c|c|c|c|c|}
\hline \multirow[b]{3}{*}{$\begin{array}{l}\text { Annual Miles } \\
\text { Traveled }\end{array}$} & \multicolumn{5}{|c|}{ Commodity-Carrying Status } & \multirow[b]{3}{*}{$\begin{array}{l}\text { TOTALS } \\
\text { Tructs }\end{array}$} \\
\hline & \multicolumn{2}{|c|}{ Commodity-Carrying } & \multicolumn{3}{|c|}{ Non-Commodity-Carrying } & \\
\hline & $\begin{array}{l}\text { Short Haul } \\
\text { Iructs }\end{array}$ & $\begin{array}{l}\text { Long Haul } \\
\text { Irucks }\end{array}$ & $\frac{\text { Business Use }}{\text { Irucks }}$ & $\frac{\text { Personal Use }}{\text { Trucks }}$ & $\frac{\text { Not In Use }}{\text { Trucks }}$ & \\
\hline \multirow[t]{2}{*}{ Leas than 5,000} & 2,175 & 63 & 1,577 & 8,200 & 64 & 12,079 \\
\hline & $(29.5 \%)$ & $(8.2 \%)$ & $(24.7 \%)$ & $(30.2 \%)$ & (54.3\%) & (229\%) \\
\hline \multirow[t]{2}{*}{$5,000-9,95$} & 1,596 & 69 & 1,477 & 6,598 & 49 & 9,789 \\
\hline & $(21.7 \%)$ & $(9.0 \%)$ & (23.1\%) & (24.3\%) & (41.3\%) & $(2348)$ \\
\hline \multirow[t]{2}{*}{$10,000-14,999$} & 1,318 & 83 & 1,718 & 7,127 & 3 & 10,249 \\
\hline & $(17.9 \%)$ & $(10.8 \%)$ & $(26.9 \%)$ & $(26.3 \%)$ & $(28 \%)$ & (24.5\%) \\
\hline \multirow[t]{2}{*}{$15,000-19,999$} & 713 & 53 & 483 & 2,508 & 0 & $\mathbf{3 , 7 5 7}$ \\
\hline & $(9.7 \%)$ & $(6.9 \%)$ & $(7.5 \%)$ & $(9.2 \%)$ & $(0.0 \%)$ & $(9.0 \%)$ \\
\hline \multirow[t]{2}{*}{$20,000-24,999$} & 530 & 37 & 402 & 1,492 & 1 & 2,462 \\
\hline & $(7.2 \%)$ & $(4.8 \%)$ & $(6.3 \%)$ & $(5.5 \%)$ & $(1.2 \%)$ & $(5.9 \%)$ \\
\hline \multirow[t]{2}{*}{$25,000-29,999$} & 243 & 36 & 141 & 738 & 0 & 1,158 \\
\hline & (3.3\%) & $(4.7 \%)$ & $(22 \%)$ & $(27 \%)$ & $(0.0 \%)$ & (28x) \\
\hline \multirow[t]{2}{*}{$30,000-39,999$} & 343 & 38 & 250 & 189 & 0 & 819 \\
\hline & $(4.7 \%)$ & $(4.9 \%)$ & $(3.9 \%)$ & $(0.7 \%)$ & $(0.0 \%)$ & $(20 x)$ \\
\hline \multirow[t]{2}{*}{$40,000-49,999$} & 162 & 68 & 128 & 103 & 1 & 463 \\
\hline & $(22 \%)$ & (8.9\%) & $(20 \%)$ & $(0.4 \%)$ & $(0.5 \%)$ & (1.1\%) \\
\hline \multirow[t]{2}{*}{$50,000-74,599$} & 169 & 90 & 98 & 125 & $\mathbf{0}$ & 481 \\
\hline & $(2.3 \%)$ & $(11.7 \%)$ & (1.5\%) & $(0.5 \%)$ & $(0.0 \%)$ & $(1.2 \%)$ \\
\hline \multirow[t]{2}{*}{$75,000-99,999$} & 55 & 97 & 62 & 45 & 0 & 259 \\
\hline & $(0.7 \%)$ & $(126 \%)$ & $(1.0 \%)$ & $(0.2 \%)$ & $(0.0 \%)$ & $(a, 6 \%)$ \\
\hline \multirow[t]{2}{*}{$100,000-124,999$} & 42 & 86 & 9 & 0 & 0 & 137 \\
\hline & $(0.6 \%)$ & (11.2\%) & $(0.1 \%)$ & $(0.0 \%)$ & $(0.0 \%)$ & $(0.3 \%)$ \\
\hline \multirow[t]{2}{*}{$125,000-145,999$} & 4 & 22 & 12 & 0 & 0 & 37 \\
\hline & $(0.1 \%)$ & $(29 \%)$ & $(0.2 \%)$ & $(0.0 \%)$ & $(0.0 \%)$ & $(a, 1 \%)$ \\
\hline \multirow[t]{2}{*}{$150,000-199,999$} & 5 & 22 & 0 & 0 & 0 & 27 \\
\hline & $(0.1 \%)$ & $(29 \%)$ & $(0.0 \%)$ & $(0.0 \%)$ & $(0.0 \%)$ & (a.1\%) \\
\hline \multirow[t]{2}{*}{200,000 and over } & 9 & 4 & 38 & 0 & 0 & 51 \\
\hline & $(C .1 \%)$ & $(0.5 \%)$ & $(0.6 \%)$ & $(0.0 \%)$ & $(0.0 \%)$ & $(a, 8)$ \\
\hline \multirow[t]{2}{*}{ TOTALS } & 7,363 & 766 & 6,393 & 27,125 & 119 & 41,768 \\
\hline & $(1000 \%)$ & $(1000 \%)$ & $(1000 \%)$ & $(100.0 \%)$ & $(1000 \%)$ & $(100.0 \%)$ \\
\hline
\end{tabular}


Table 219

Number of Four Year and Older Trucks

by Annual Miles Traveled and Truck Type: 1990 NTACS

(Trucks in Thousands)

\begin{tabular}{|c|c|c|c|c|c|c|}
\hline \multirow{2}{*}{$\begin{array}{l}\text { Annual Miles } \\
\text { Traveled }\end{array}$} & \multicolumn{5}{|c|}{ Truck Type } & \multirow[b]{2}{*}{ TOTALS } \\
\hline & $\begin{array}{l}\text { Pickup } \\
\text { Trucks }\end{array}$ & $\begin{array}{l}\text { Utility } \\
\text { Trucks }\end{array}$ & $\begin{array}{l}\text { Small } \\
\text { Trucks }\end{array}$ & $\begin{array}{c}\text { Large } \\
\text { Irucks }\end{array}$ & $\begin{array}{l}\text { Truck- } \\
\text { Tractor } \\
\text { Trucks }\end{array}$ & \\
\hline \multirow[t]{2}{*}{ Less than 5,000} & 7,758 & 2,593 & 1,296 & 282 & 151 & 12,079 \\
\hline & (31.6\%) & (21.1\%) & (45.5\%) & $(27.0 \%)$ & (14.4\%) & (289\%) \\
\hline \multirow[t]{2}{*}{$5,000-9,999$} & 6,437 & 2,549 & 555 & 163 & 85 & 9,789 \\
\hline & $(26.2 \%)$ & $(20.7 \%)$ & (19.5\%) & $(15.6 \%)$ & $(8.1 \%)$ & $(234 \%)$ \\
\hline \multirow[t]{2}{*}{$10,000-14,999$} & 5,581 & 4,022 & 413 & 163 & 70 & 10,249 \\
\hline & (227\%) & $(327 \%)$ & $(14.5 \%)$ & $(15.6 \%)$ & $(6.7 \%)$ & (24.5\%) \\
\hline \multirow[t]{2}{*}{$15,000-19,999$} & 1,962 & 1,440 & 220 & 84 & $\$ 1$ & 3,757 \\
\hline & $(8.0 \%)$ & (11.7\%) & (7.7\%) & $(8.0 \%)$ & $(4.8 \%)$ & $(9.0 \%)$ \\
\hline \multirow[t]{2}{*}{$20,000-24,999$} & 1,253 & 954 & 120 & 85 & 50 & 2,462 \\
\hline & $(5.1 \%)$ & $(7.8 \%)$ & $(4.2 \%)$ & (8.1\%) & $(4.8 \%)$ & (5.9x) \\
\hline \multirow[t]{2}{*}{$25,000-29,999$} & 665 & 342 & 66 & 41 & 44 & 1,158 \\
\hline & $(27 \%)$ & $(28 \%)$ & $(2.3 \%)$ & $(4.0 \%)$ & $(4.2 \%)$ & (28\%) \\
\hline \multirow[t]{2}{*}{$30,000-39,999$} & 373 & 209 & 65 & 87 & 85 & 819 \\
\hline & $(1.5 \%)$ & $(1.7 \%)$ & (23\%) & (8.4\%) & $(8.1 \%)$ & $\left(20 x_{0}\right)$ \\
\hline \multirow[t]{2}{*}{$40,000-49,999$} & 138 & 147 & 56 & 47 & 74 & 463 \\
\hline & $(0.0 \%)$ & $(1.2 \%)$ & $(2.0 \%)$ & $(4.5 \%)$ & $(7.0 \%)$ & (1.1\%) \\
\hline \multirow[t]{2}{*}{$50,000-74,999$} & 212 & 24 & 34 & 56 & 155 & 481 \\
\hline & $(0.9 \%)$ & $(0.2 \%)$ & $(1.2 \%)$ & $(5.4 \%)$ & $(14.7 \%)$ & (1.2\%) \\
\hline \multirow[t]{2}{*}{$75,000-99,999$} & 106 & 2 & 4 & 22 & 125 & 259 \\
\hline & $(0.4 \%)$ & (a) & $(0.2 \%)$ & $(2.1 \%)$ & (11.9\%) & (a.6\%) \\
\hline \multirow[t]{2}{*}{$100,000-124,999$} & 14 & 7 & 2 & 10 & 104 & 137 \\
\hline & $(0.1 \%)$ & $(0.1 \%)$ & $(0.1 \%)$ & $(0.9 \%)$ & $(9.9 \%)$ & (a.3\%) \\
\hline \multirow[t]{2}{*}{$125,000-149,999$} & 0 & 0 & 12 & 3 & 23 & 37 \\
\hline & $(0.0 \%)$ & $(0.0 \%)$ & $(0.4 \%)$ & $(0.3 \%)$ & $(22 \%)$ & (a.1\%) \\
\hline \multirow[t]{2}{*}{$150,000-199,999$} & 0 & 0 & 0 & 1 & 26 & 27 \\
\hline & $(0.0 \%)$ & $(0.0 \%)$ & $(0.0 \%)$ & $(0.1 \%)$ & $(25 \%)$ & (a.1\%) \\
\hline \multirow[t]{2}{*}{200,000 and over } & 38 & 0 & 4 & $\mathbf{a}$ & 8 & 51 \\
\hline & $(0.2 \%)$ & $(0.0 \%)$ & $(0.1 \%)$ & (a) & $(0.8 \%)$ & (a.1\%) \\
\hline \multirow[t]{2}{*}{ TOTALS } & 24,537 & 12,290 & 2,847 & 1,044 & 1,050 & 41,768 \\
\hline & $(1000 \%)$ & $(1000 \%)$ & (1000\%) & $(100.0 \%)$ & (100.0\%) & $(1000 \%)$ \\
\hline
\end{tabular}

(a) Quantity rounded to zero. 
Table 220

Number of Four Year and Older Trucks

by Annual Miles Traveled and Major Use: 1990 NTACS

(Trucks in Thousands)

\begin{tabular}{|c|c|c|c|c|c|c|}
\hline \multirow[b]{2}{*}{$\begin{array}{l}\text { Annisal Miles } \\
\text { Traveled }\end{array}$} & \multicolumn{5}{|c|}{ Major Use } & \multirow[b]{2}{*}{$\begin{array}{l}\text { TOTALS } \\
\text { Truch }\end{array}$} \\
\hline & $\begin{array}{c}\text { Personal } \\
\text { Transportation } \\
\text { Irucks } \\
\end{array}$ & $\begin{array}{l}\text { Contract } \\
\text { Carrier } \\
\text { Irucks }\end{array}$ & $\begin{array}{c}\text { Common } \\
\text { Carrier } \\
\text { Trucks } \\
\end{array}$ & $\begin{array}{c}\text { Other } \\
\text { Business Use } \\
\frac{\text { Trucks }}{}\end{array}$ & $\frac{\text { Unknown }}{\text { Trucks }}$ & \\
\hline \multirow[t]{2}{*}{ Less than 5,000 } & 9,232 & 69 & 250 & 2,105 & 423 & 12,079 \\
\hline & $(29.2 \%)$ & $(10.6 \%)$ & $(17.6 \%)$ & $(28.6 \%)$ & $(57.1 \%)$ & $(289 \times 5)$ \\
\hline \multirow[t]{2}{*}{$5,000-9,999$} & 7,779 & 91 & 246 & 1,520 & 152 & 9,789 \\
\hline & $(24.6 \%)$ & (13.9\%) & $(17.4 \%)$ & $(20.7 \%)$ & $(20.6 \%)$ & $(23.4 \%)$ \\
\hline \multirow[t]{2}{*}{$10,000-14,999$} & 8,296 & 131 & 196 & 1,578 & 49 & 10,249 \\
\hline & $(26.2 \%)$ & (19.9\%) & $(13.8 \%)$ & $(21.5 \%)$ & $(6.6 \%)$ & $(24.5 \%)$ \\
\hline \multirow[t]{2}{*}{$15,000-19,999$} & 3,038 & 25 & 90 & 590 & 14 & 3,757 \\
\hline & $(9.6 \%)$ & $(3.8 \%)$ & $(6.3 \%)$ & $(8.0 \%)$ & $(1.9 \%)$ & $(9.0 \%)$ \\
\hline \multirow[t]{2}{*}{$20,000-24,999$} & 1,580 & 81 & 133 & 579 & 90 & 2,462 \\
\hline & $(5.0 \%)$ & $(123 \%)$ & $(9.4 \%)$ & (7.9\%) & $(121 \%)$ & (5.9\%) \\
\hline \multirow[t]{2}{*}{$25,000-29,999$} & 837 & 27 & 95 & 197 & 2 & 1,158 \\
\hline & $(26 \%)$ & $(4.1 \%)$ & $(6.7 \%)$ & $(27 \%)$ & $(0.2 \%)$ & $(28 \%)$ \\
\hline \multirow[t]{2}{*}{$30,000-39,999$} & 380 & 39 & 73 & 323 & 5 & 819 \\
\hline & $(1.2 \%)$ & $(0.0 \%)$ & $(5.1 \%)$ & $(4.4 \%)$ & $(0.6 \%)$ & $(20 \%)$ \\
\hline \multirow[t]{2}{*}{$40,000-49,999$} & 224 & 32 & 88 & 116 & 2 & 463 \\
\hline & $(0.7 \%)$ & $(4.8 \%)$ & $(6.2 \%)$ & $(1.6 \%)$ & $(0.3 \%)$ & (1.1\%) \\
\hline \multirow[t]{2}{*}{$50,000-74,999$} & 139 & 55 & 87 & 198 & 2 & 481 \\
\hline & $(0.4 \%)$ & $(8.4 \%)$ & $(6.1 \%)$ & $(27 \%)$ & $(0.2 \%)$ & $(1.2 \%)$ \\
\hline \multirow[t]{2}{*}{$75,000-99,999$} & 51 & 48 & 64 & 95 & 2 & 259 \\
\hline & $(0.2 \%)$ & $(7.3 \%)$ & $(4.5 \%)$ & $(1.3 \%)$ & $(0.2 \%)$ & $(0.6 \%)$ \\
\hline \multirow[t]{2}{*}{$100,000-124,999$} & 10 & 43 & 58 & 26 & $\mathbf{a}$ & 137 \\
\hline & (a) & $(0.5 \%)$ & $(4.1 \%)$ & $(0.3 \%)$ & (a) & (a3\%) \\
\hline \multirow[t]{2}{*}{$12:, 900-1.49,999$} & 0 & 8 & 15 & 14 & a & 37 \\
\hline & $(0.0 \%)$ & $(1.2 \%)$ & $(1.1 \%)$ & $(0.2 \%)$ & (a) & $(a 1 \%)$ \\
\hline \multirow[t]{2}{*}{$150,000-199,999$} & 0 & 6 & 19 & 2 & 0 & $\boldsymbol{2 1}$ \\
\hline & $(0.0 \%)$ & $(0.9 \%)$ & $(1.3 \%)$ & (a) & $(0.0 \%)$ & $(a .15)$ \\
\hline \multirow[t]{2}{*}{200,000 and over } & 38 & 3 & 5 & 5 & 0 & 51 \\
\hline & $(0.1 \%)$ & $(0.4 \%)$ & $(0.4 \%)$ & $(0.1 \%)$ & $(0.0 \%)$ & $(a 1 \%)$ \\
\hline \multirow[t]{2}{*}{ TOTALS } & 31,605 & 656 & 1,419 & 7,347 & 741 & 41,768 \\
\hline & $(1000 \%)$ & $(1000 \times)$ & $(1000 \%)$ & $(100.0 \%)$ & $(100.0 \%)$ & $(1000 \%)$ \\
\hline
\end{tabular}

(a) Quantity rounded to zero. 
Table 221

Number of Four Year and Older Trucks

by Annual Miles Traveled and Truck Description: 1990 NTACS

(Trucks in Thousands)

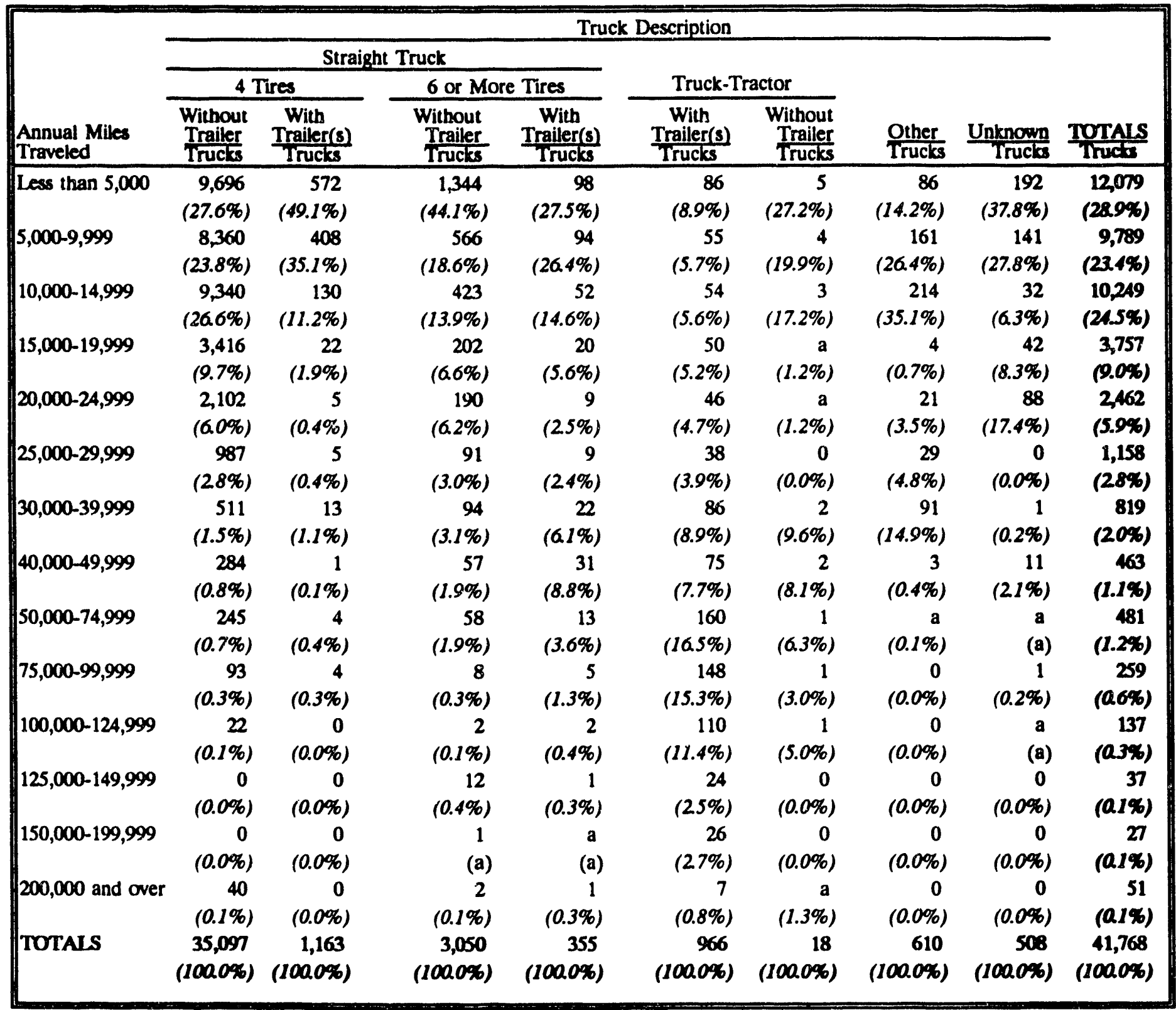

(a) Quantity rounded to zero. 
Table 2.22a

Distribution of Four Year and Older Trucks That Operated in Canada by Commodity-Carrying Status: 1990 NTACS

\begin{tabular}{|c|c|c|c|c|}
\hline \multicolumn{4}{|c|}{ Commodity-Carrying Status } & \multirow[b]{3}{*}{$\frac{\text { TOTALS }}{\text { Trucks }}$} \\
\hline \multicolumn{2}{|c|}{ Commodity-Carrying } & \multicolumn{2}{|c|}{ Non-Commodity-Carrying } & \\
\hline$\frac{\text { Short Haul }}{\text { Trucks }}$ & $\frac{\text { Long Haul }}{\text { Trucks }}$ & $\frac{\text { Business Use }}{\text { Trucks }}$ & $\frac{\text { Personal Use }}{\text { Trucks }}$ & \\
\hline $\begin{array}{l}48,384 \\
(84 \%)\end{array}$ & $\begin{array}{l}43,776 \\
(7.6 \%)\end{array}$ & $\begin{array}{l}38,592 \\
(6.7 \%)\end{array}$ & $\begin{array}{l}445,250 \\
(77.3 \%)\end{array}$ & $\begin{array}{l}576,003 \\
(100.0 \%)\end{array}$ \\
\hline
\end{tabular}

Table 222b

Distribution of Four Year and Older Trucks That Operated in Mexico by Commodity-Carrying Status: 1990 NTACS

\begin{tabular}{|c|c|c|c|c|}
\hline \multicolumn{4}{|c|}{ Commodity-Carrying Status } & \multirow[b]{3}{*}{$\frac{\text { TOTALS }}{\text { Trucks }}$} \\
\hline \multicolumn{2}{|c|}{ Commodity-Carrying } & \multicolumn{2}{|c|}{ Non-Commodity-Carrying } & \\
\hline$\frac{\text { Short Haul }}{\text { Trucks }}$ & $\frac{\text { Lone Haul }}{\text { Trucks }}$ & $\frac{\text { Business Use }}{\text { Trucks }}$ & $\frac{\text { Personal Use }}{\text { Trucks }}$ & \\
\hline $\begin{array}{c}71,834 \\
(34.9 \%)\end{array}$ & $\begin{array}{c}3,087 \\
(1.5 \%)\end{array}$ & $\begin{array}{c}0 \\
(0.0 \%)\end{array}$ & $\begin{array}{l}131,524 \\
(63.9 \%)\end{array}$ & $\begin{array}{l}205,828 \\
(100.0 \%)\end{array}$ \\
\hline
\end{tabular}


Table 223a

Distribution of Four Year and Older Trucks That Operated in Canada by Truck Type: 1990 NTACS

\begin{tabular}{|c|c|c|c|c|c|}
\hline \multicolumn{5}{|c|}{ Truck Type } & \multirow[b]{2}{*}{$\frac{\text { TOTALS }}{\text { Trucks }}$} \\
\hline $\begin{array}{l}\text { Pickup } \\
\text { Trucks }\end{array}$ & $\begin{array}{l}\text { Utility } \\
\text { Trucks }\end{array}$ & $\frac{\text { Small }}{\text { Trucks }}$ & $\begin{array}{l}\text { Large } \\
\text { Trucks }\end{array}$ & $\frac{\text { Truck-Tractor }}{\text { Trucks }}$ & \\
\hline 281,089 & 235,585 & 14,400 & 5,184 & 39,744 & 576,003 \\
\hline$(48.8 \%)$ & $(40.9 \%)$ & $(2.5 \%)$ & $(0.9 \%)$ & $(6.9 \%)$ & $(10.0 .0 \%)$ \\
\hline
\end{tabular}

Table 2.236

Distribution of Four Year and Older Trucks That Operated in Merico by Truck Type: 1990 NTACS

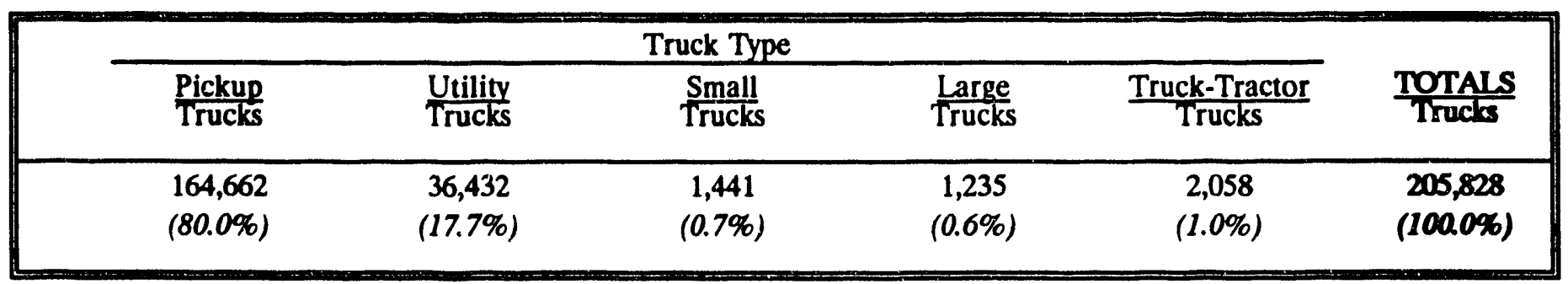


Table 2.24a

Distribution of Four Year and Older Trucks That Operated in Canada by Major Use: 1990 NTACS

\begin{tabular}{|c|c|c|c|c|c|}
\hline \multicolumn{5}{|c|}{ Major Use } & \multirow[b]{2}{*}{$\frac{\text { TOTALS }}{\text { Iruch }}$} \\
\hline $\begin{array}{c}\text { Personal } \\
\text { Transportation } \\
\text { Irucks }\end{array}$ & $\begin{array}{l}\text { Contract } \\
\text { Carrier } \\
\text { Trucks }\end{array}$ & $\begin{array}{l}\text { Common } \\
\text { Carrier } \\
\text { Trucks } \\
\end{array}$ & $\begin{array}{c}\text { Other } \\
\text { Business Use } \\
\frac{\text { Trucks }}{\text { Trust }}\end{array}$ & $\frac{\text { Unknown }}{\text { Trucks }}$ & \\
\hline 496,515 & 13,248 & 24,768 & 33,984 & 8,064 & 576,003 \\
\hline$(86.2 \%)$ & $(2.3 \%)$ & $(4.3 \%)$ & $(5.9 \%)$ & $(1.4 \%)$ & $(100.0 \%)$ \\
\hline
\end{tabular}

Table 224b

Distribution of Four Year and Older Trucks That Operated in Mexico by Major Use: 1990 NTACS

\begin{tabular}{|ccccc|}
\hline \multicolumn{4}{c}{ Major Use } & \\
\hline $\begin{array}{c}\text { Personal } \\
\text { Transportation }\end{array}$ & $\begin{array}{c}\text { Contract } \\
\text { Carrier }\end{array}$ & $\begin{array}{c}\text { Common } \\
\text { Carrier }\end{array}$ & $\begin{array}{c}\text { Other } \\
\text { Business Use }\end{array}$ & TOTALs \\
\hline Trucks & Trucks & Trucks & $\frac{\text { Trucks }}{\text { Truck }}$ \\
\hline 172,896 & 412 & 5,146 & 27,581 & 205,828 \\
$(84.0 \%)$ & $(0.2 \%)$ & $(2.5 \%)$ & $(13.4 \%)$ & $(100.0 \%)$ \\
\hline
\end{tabular}




\section{CHAPTER 3}

\section{DAILY DESCRIPTIONS AND ACTIVITIES OF THE 1990 NTACS TRUCK POPULATION}

\section{IMPORTANT NOTICE}

All estimates in every table of this report have been adjusted using the three stratifying variables: (i) commodity-carrying status, (ii) census division, and (iii) truck type to compensate for extremely high nonresponse rates. (See Tables $\mathbf{G}$ and $\mathbf{H}$ of Appendix A). As a resuit, preliminary comparisons with other data sources have revealed inconsistencies -especially when detailed estimates are presented using variables other than the three stratifying variables. Individual estimates are not highly reliable and should be used cautiously only to give impressions of broad trends, patterns, and distributions. 
Table 3.1

Number of Four Year and Older Trucks and Associated Daily Vehicle Miles Traveled (DVMT) by Truck Model Year: 1990 NTACS

(Trucks in Thousands/Daily VMT in Millions)

\begin{tabular}{|lrcrrr|}
\hline Truck Model Year & \multicolumn{2}{c}{ Trucks } & \multicolumn{3}{c|}{ Daily VMT } \\
\hline $1987(\mathrm{a})$ & 3,815 & $(9.1 \%)$ & 200 & $(10.7 \%)$ \\
1986 & 5,601 & $(13.4 \%)$ & 318 & $(17.0 \%)$ \\
1985 & 4,163 & $(10.0 \%)$ & 204 & $(10.9 \%)$ \\
1984 & 3,667 & $(8.8 \%)$ & 191 & $(10.2 \%)$ \\
1983 & 3,210 & $(7.7 \%)$ & 139 & $(7.4 \%)$ \\
1982 & 2,411 & $(5.8 \%)$ & 100 & $(5.3 \%)$ \\
1981 & 1,807 & $(4.3 \%)$ & 73 & $(3.9 \%)$ \\
1980 & 1,217 & $(2.9 \%)$ & 54 & $(2.9 \%)$ \\
1979 & 3,205 & $(7.7 \%)$ & 106 & $(5.7 \%)$ \\
1978 & 2,475 & $(5.9 \%)$ & 116 & $(6.2 \%)$ \\
Pre-1978 & 10,194 & $(24.4 \%)$ & 371 & $(19.8 \%)$ \\
Unknown & 3 & $(\mathrm{~b})$ & $\mathrm{b}$ & $(\mathrm{b})$ \\
TOOTALs & 41,768 & $(100.0 \%)$ & 1,872 & $(100.0 \%)$ \\
& & & & & \\
\hline
\end{tabular}

(a) Includes some 1988 models.

(b) Quantity rounded to zero. 
Table 3.2

Number of Four Year and Older Trucks and Associated Daily Vehicle Miles Traveled (DVMT) by Commodity-Carrying Status: 1990 NTACS

(Trucks in Thousands/Daily VMT in Millions)

\begin{tabular}{|lrrrr|}
\hline $\begin{array}{l}\text { Commodity } \\
\text { Carrying Status }\end{array}$ & \multicolumn{2}{c}{ Trucks } & Daily VMT \\
\hline Commodity-Carrying & & & & \\
- Short Haul & 7,365 & $(17.6 \%)$ & 457 & $(24.4 \%)$ \\
- Long Haul & 766 & $(1.8 \%)$ & 176 & $(9.4 \%)$ \\
& & & & \\
Non-Commodity-Carrying & & & & \\
- Business Use & 6,393 & $(15.3 \%)$ & 274 & $(14.6 \%)$ \\
- Personal Use & 27,125 & $(64.9 \%)$ & 963 & $(51.4 \%)$ \\
- Not In Use & 119 & $(0.3 \%)$ & 3 & $(0.2 \%)$ \\
TOTALS & 41,768 & $(100.0 \%)$ & 1,872 & $(100.0 \%)$ \\
& & & & \\
\hline
\end{tabular}


Table 3.3

Number of Four Year and Older Trucles and Associated Daily Vehicle Miles Traveled (DVMT) by Truck Type: 1990 NTACS

(Trucks in Thousands/Daily VMT in Millions)

\begin{tabular}{|c|c|c|c|c|}
\hline \multirow{2}{*}{$\frac{\text { Truck Type }}{\text { Pickup }}$} & \multicolumn{2}{|c|}{ Trucks } & \multicolumn{2}{|c|}{ Daily VMT } \\
\hline & 24,537 & $(58.7 \%)$ & 893 & $(47.7 \%)$ \\
\hline Utility & 12,290 & $(29.4 \%)$ & 493 & (26.3\%) \\
\hline Small & 2,847 & $(6.8 \%)$ & 149 & $(8.0 \%)$ \\
\hline Large & 1,044 & $(2.5 \%)$ & 103 & $(5.5 \%)$ \\
\hline Truck-Tractor & 1,050 & $(2.5 \%)$ & 233 & (12.5\%) \\
\hline TOTALS & 41,768 & $(100.0 \%)$ & 1,872 & $(100.0 \%)$ \\
\hline
\end{tabular}




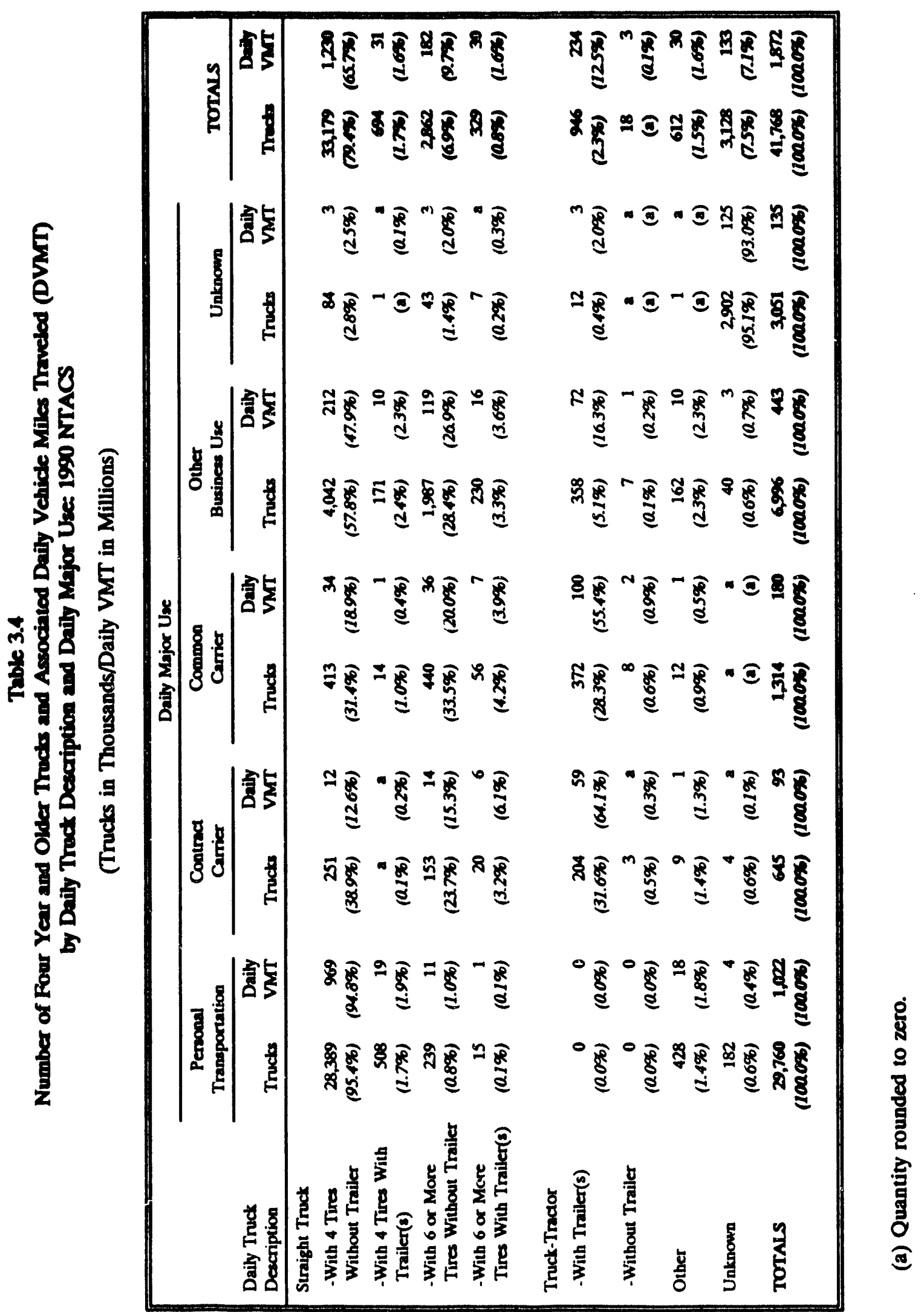


Table 3.5

Number of Four Year and Older Trucks and Associated Daily Vehicle Miles Traveled (DVMT) by Daily Fuel Use and Daily Truck Description: 1990 NTACS

(Trucks in Thousands/Daily VMT in Millions)

\begin{tabular}{|c|c|c|c|c|c|c|c|c|c|}
\hline \multirow[b]{4}{*}{$\begin{array}{l}\text { Daily } \\
\text { Fuel Use } \\
\text { (Gallons) }\end{array}$} & \multicolumn{8}{|c|}{ Daily Truck Desciption } & \multirow[b]{4}{*}{$\frac{\text { TOTALS }}{\text { Truats }}$} \\
\hline & \multicolumn{4}{|c|}{ Straight Truck } & & & \multirow[b]{3}{*}{$\frac{\text { Other }}{\text { Trucks }}$} & \multirow[b]{3}{*}{$\frac{\text { Unknown }}{\text { Trucks }}$} & \\
\hline & \multicolumn{2}{|c|}{4 Tires } & \multicolumn{2}{|c|}{6 or More Tires } & \multicolumn{2}{|c|}{ Truck-Tractor } & & & \\
\hline & $\begin{array}{l}\text { Without } \\
\text { Trailor } \\
\text { Trucks }\end{array}$ & $\begin{array}{c}\text { With } \\
\text { Trailor(s) } \\
\text { Trucks }\end{array}$ & $\begin{array}{l}\text { Without } \\
\text { Trailor } \\
\text { Trucks }\end{array}$ & $\begin{array}{c}\text { With } \\
\text { Trailor(s) } \\
\text { Trucks }\end{array}$ & $\begin{array}{c}\text { With } \\
\text { Trailor(s) } \\
\text { Trucks }\end{array}$ & $\begin{array}{l}\text { Without } \\
\text { Trailor } \\
\text { Trucks }\end{array}$ & & & \\
\hline \multirow[t]{2}{*}{ Less Than 20} & 32,598 & 676 & 2,360 & 230 & 240 & 10 & 576 & 2,951 & 39,641 \\
\hline & $(98.2 \%)$ & (97.5\%) & $(825 \%)$ & $(69.9 \%)$ & $(25.4 \%)$ & $(524 \%)$ & $(94.1 \%)$ & $(94.3 \%)$ & $(949 \% 5)$ \\
\hline \multirow[t]{2}{*}{$20-39$} & 457 & 14 & 346 & 65 & 205 & 6 & 32 & 154 & 1,279 \\
\hline & $(1.4 \%)$ & $(20 \%)$ & $(12.1 \%)$ & $(19.7 \%)$ & $(21.6 \%)$ & $(34.1 \%)$ & $(5.2 \%)$ & $(4.9 \%)$ & $(31 \%)$ \\
\hline \multirow[t]{2}{*}{$40-59$} & 92 & 3 & 96 & 11 & 165 & $\mathbf{a}$ & 4 & 11 & 383 \\
\hline & $(0.3 \%)$ & $(0.4 \%)$ & $(3.4 \%)$ & $(3.4 \%)$ & $(17.5 \%)$ & $(1.2 \%)$ & $(0.6 \%)$ & $(0.3 \%)$ & $(a 9 \% b)$ \\
\hline \multirow[t]{2}{*}{$60-79$} & 1 & $\mathbf{a}$ & 18 & 10 & 142 & 1 & $\mathbf{a}$ & 4 & 176 \\
\hline & (a) & (a) & $(0.6 \%)$ & $(3.0 \%)$ & $(15.0 \%)$ & $(6.6 \%)$ & $(0.1 \%)$ & $(0.1 \%)$ & $(0.45)$ \\
\hline \multirow[t]{2}{*}{$80-99$} & 23 & 0 & 32 & 9 & 106 & $\mathbf{a}$ & 0 & 4 & 174 \\
\hline & $(0.1 \%)$ & $(0.0 \%)$ & $(1.1 \%)$ & $(26 \%)$ & $(11.2 \%)$ & $(0.7 \%)$ & $(0.0 \%)$ & $(0.1 \%)$ & $(a+45)$ \\
\hline \multirow[t]{2}{*}{$100-119$} & 0 & $\mathbf{a}$ & 5 & 3 & 51 & 1 & 0 & 3 & 63 \\
\hline & $(0.0 \%)$ & (a) & $(0.2 \%)$ & $(0.8 \%)$ & $(5.4 \%)$ & $(4.3 \%)$ & $(0.0 \%)$ & $(0.1 \%)$ & $(a 295)$ \\
\hline \multirow[t]{2}{*}{120 and Over } & 9 & 0 & 4 & 2 & 37 & $\mathbf{a}$ & 0 & $\mathbf{a}$ & 51 \\
\hline & (a) & $(0.0 \%)$ & $(0.1 \%)$ & $(0.5 \%)$ & $(3.9 \%)$ & $(0.7 \%)$ & $(0.0 \%)$ & (a) & (0.15) \\
\hline \multirow[t]{2}{*}{ TOTALS } & 33,179 & 694 & 2862 & 329 & 946 & 18 & 612 & 3,128 & 41,768 \\
\hline & $(1000 \times)$ & $(1000 \%)$ & $(1000 \%)$ & $(1000 \%)$ & $(1000 \%)$ & $(100.0 \%)$ & $(100.0 \%)$ & $(1000 \%)$ & $(1000 \%)$ \\
\hline (Gallons) & $\begin{array}{l}\text { Daily } \\
\text { VMT }\end{array}$ & $\begin{array}{l}\text { Daily } \\
\text { VMT }\end{array}$ & $\begin{array}{l}\text { Daily } \\
\text { VMT }\end{array}$ & $\begin{array}{l}\text { Daily } \\
\text { VMT }\end{array}$ & $\begin{array}{l}\text { Daily } \\
\text { VMT }\end{array}$ & $\begin{array}{l}\text { Daily } \\
\text { VMT }\end{array}$ & $\begin{array}{l}\text { Daity } \\
\text { VMT }\end{array}$ & $\begin{array}{l}\text { Daily } \\
\text { VMT }\end{array}$ & $\begin{array}{l}\text { Daily } \\
\text { VMT }\end{array}$ \\
\hline \multirow[t]{2}{*}{ Less Than 20} & 1,106 & 27 & 106 & 9 & 17 & a & 25 & 108 & 1,399 \\
\hline & $(89.9 \%)$ & $(87.3 \%)$ & $(57.9 \%)$ & (31.7\%) & (7.5\%) & $(18.6 \%)$ & $(820 \%)$ & $(81.8 \%)$ & $(747 \%)$ \\
\hline \multirow[t]{2}{*}{$20-39$} & 105 & 3 & 45 & 10 & 31 & 1 & 5 & 16 & 216 \\
\hline & (8.5\%) & $(10.2 \%)$ & (24.8\%) & $(325 \%)$ & $(13.4 \%)$ & $(48.1 \%)$ & $(15.3 \%)$ & $(120 \%)$ & (11.5\%) \\
\hline \multirow[t]{2}{*}{$40-59$} & 18 & 1 & 20 & 3 & 41 & $\mathbf{a}$ & 1 & 4 & 88 \\
\hline & $(1.5 \%)$ & $(24 \%)$ & $(11.1 \%)$ & $(9.7 \%)$ & $(17.7 \%)$ & $(1.8 \%)$ & $(22 \%)$ & $(27 \%)$ & $(4.7 \%)$ \\
\hline \multirow[t]{2}{*}{$60-79$} & $\mathbf{a}$ & $\mathbf{a}$ & 5 & 3 & 49 & $\mathbf{a}$ & a & 1 & 59 \\
\hline & (a) & (a) & $(28 \%)$ & $(10.7 \%)$ & $(20.9 \%)$ & $(17.6 \%)$ & $(0.4 \%)$ & $(1.0 \%)$ & $(32 \%)$ \\
\hline \multirow[t]{2}{*}{$80-99$} & 1 & 0 & 5 & 2 & 47 & $\mathbf{a}$ & 0 & 2 & 57 \\
\hline & (a) & $(0.0 \%)$ & $(29 \%)$ & $(8.2 \%)$ & $(20.0 \%)$ & $(2.2 \%)$ & $(0.0 \%)$ & (1.3\%) & $(30 \%)$ \\
\hline \multirow[t]{2}{*}{$100-119$} & 0 & $\mathbf{a}$ & 1 & 1 & 25 & $\mathbf{a}$ & 0 & 1 & 28 \\
\hline & $(0.0 \%)$ & (a) & $(0.3 \%)$ & $(3.1 \%)$ & $(10.7 \%)$ & (11.6\%) & $(0.0 \%)$ & $(1.1 \%)$ & (1.5\%) \\
\hline \multirow[t]{2}{*}{120 and Over } & a & 0 & 1 & 1 & 23 & a & 0 & a & 25 \\
\hline & (a) & $(0.0 \%)$ & $(0.3 \%)$ & $(4.2 \%)$ & $(9.9 \%)$ & $(0.1 \%)$ & $(0.0 \%)$ & (a) & $(1.4 \%)$ \\
\hline \multirow[t]{2}{*}{ TOTAIS } & 1,230 & 31 & 182 & 30 & 234 & 3 & 30 & 133 & 1,872 \\
\hline & (1040\%) & $(100.0 \times)$ & $(1000 \%)$ & $(1000 \%)$ & $(1000 \%)$ & $(100.0 \%)$ & $(100.0 \%)$ & $(100.0 \%)$ & $(1000 \%)$ \\
\hline
\end{tabular}

(a) Quantity rounded to zero. 


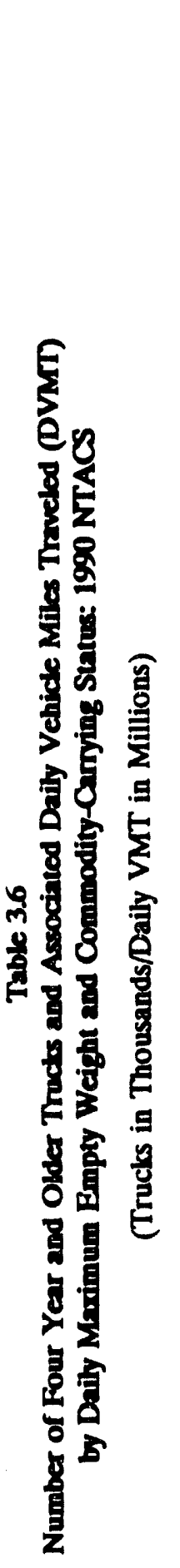

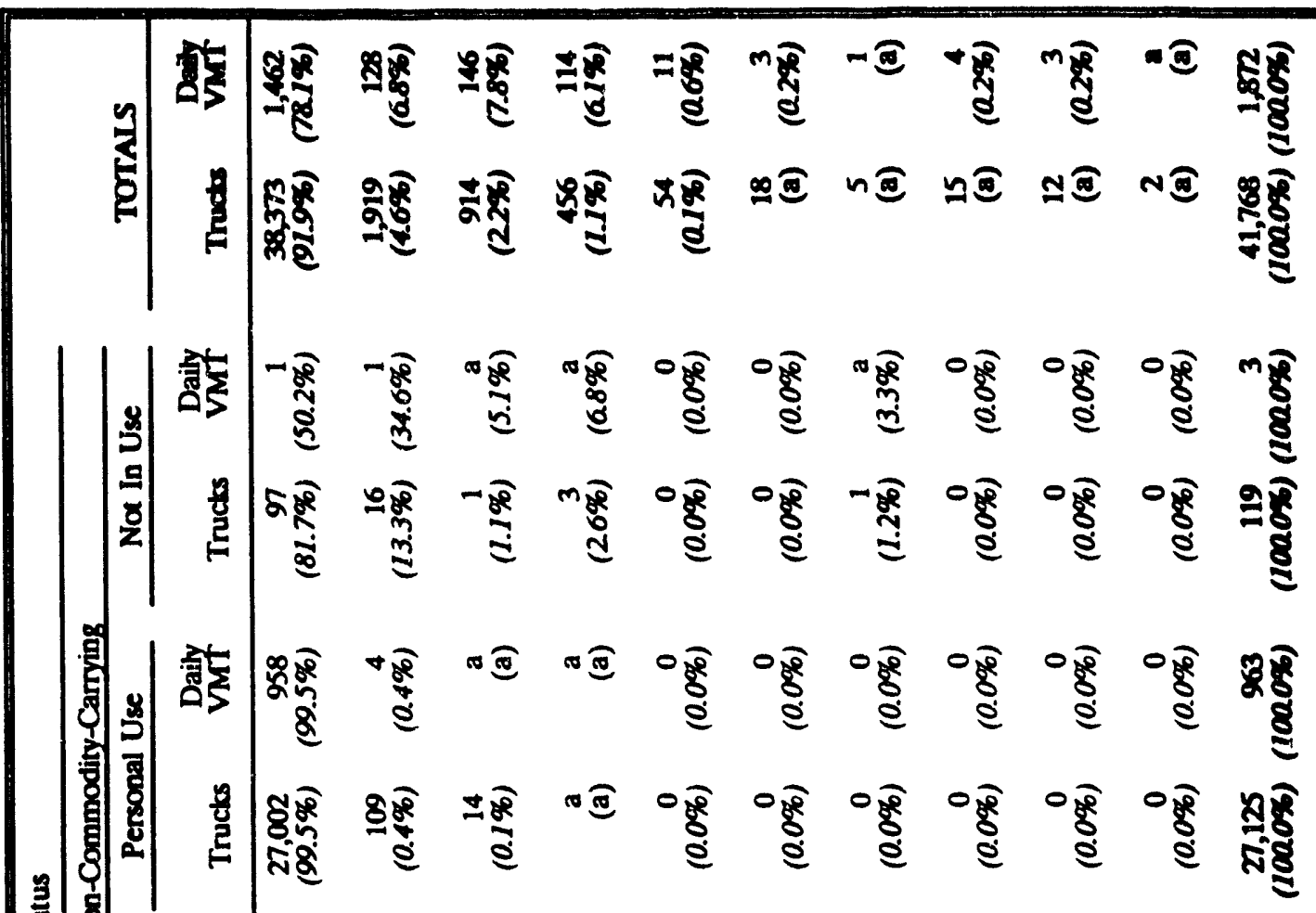

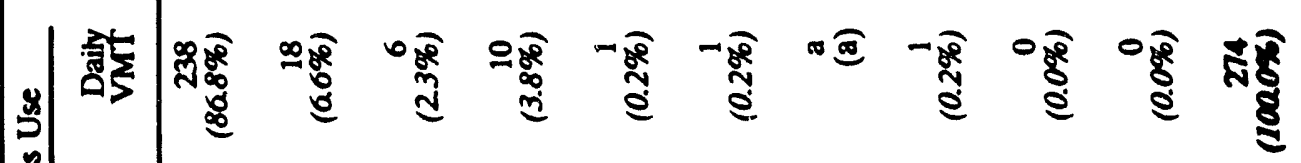

鄫 部

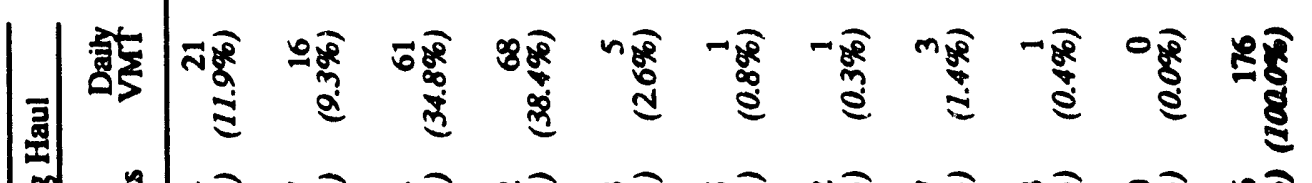

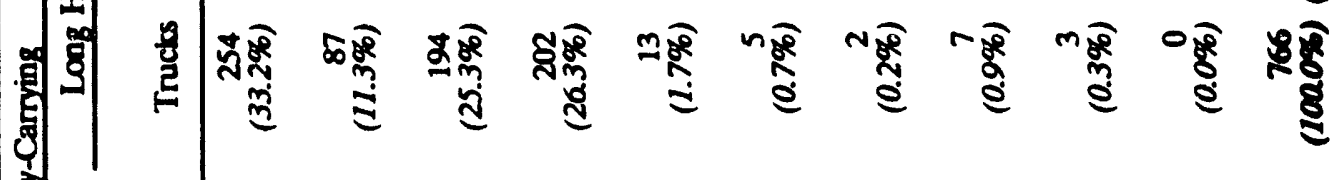

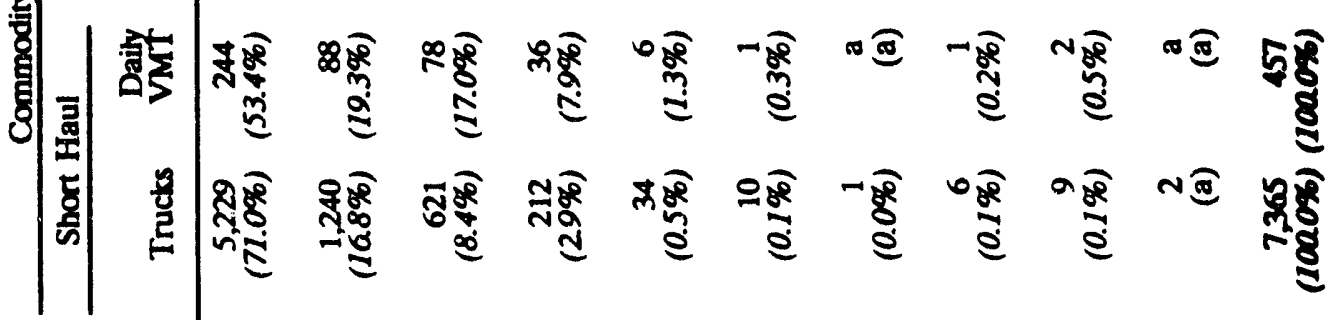

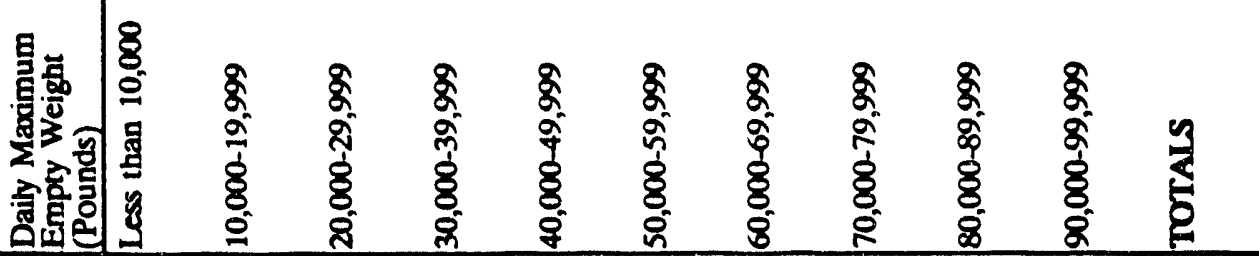

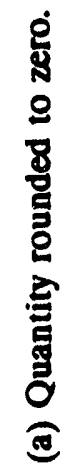




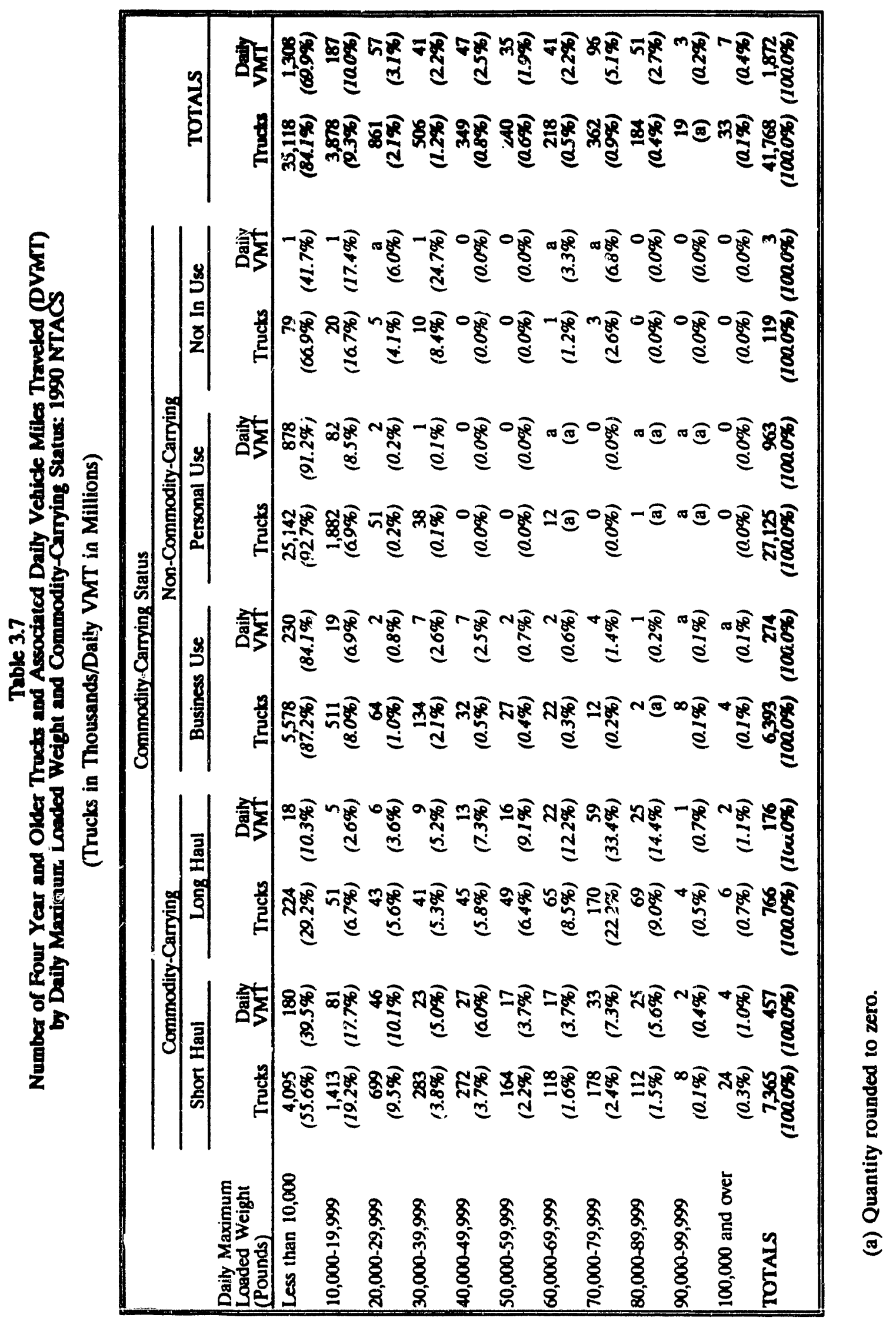




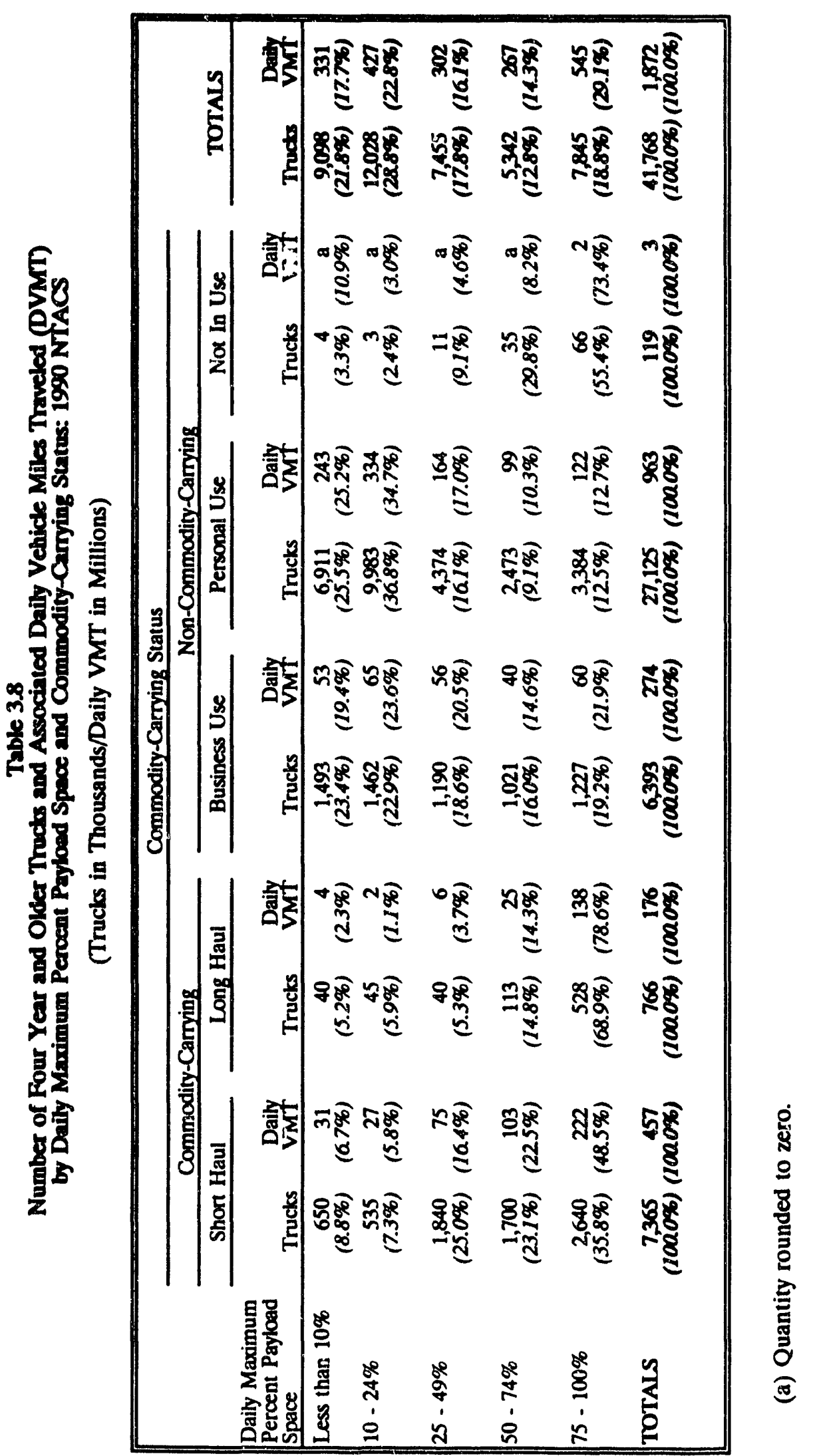




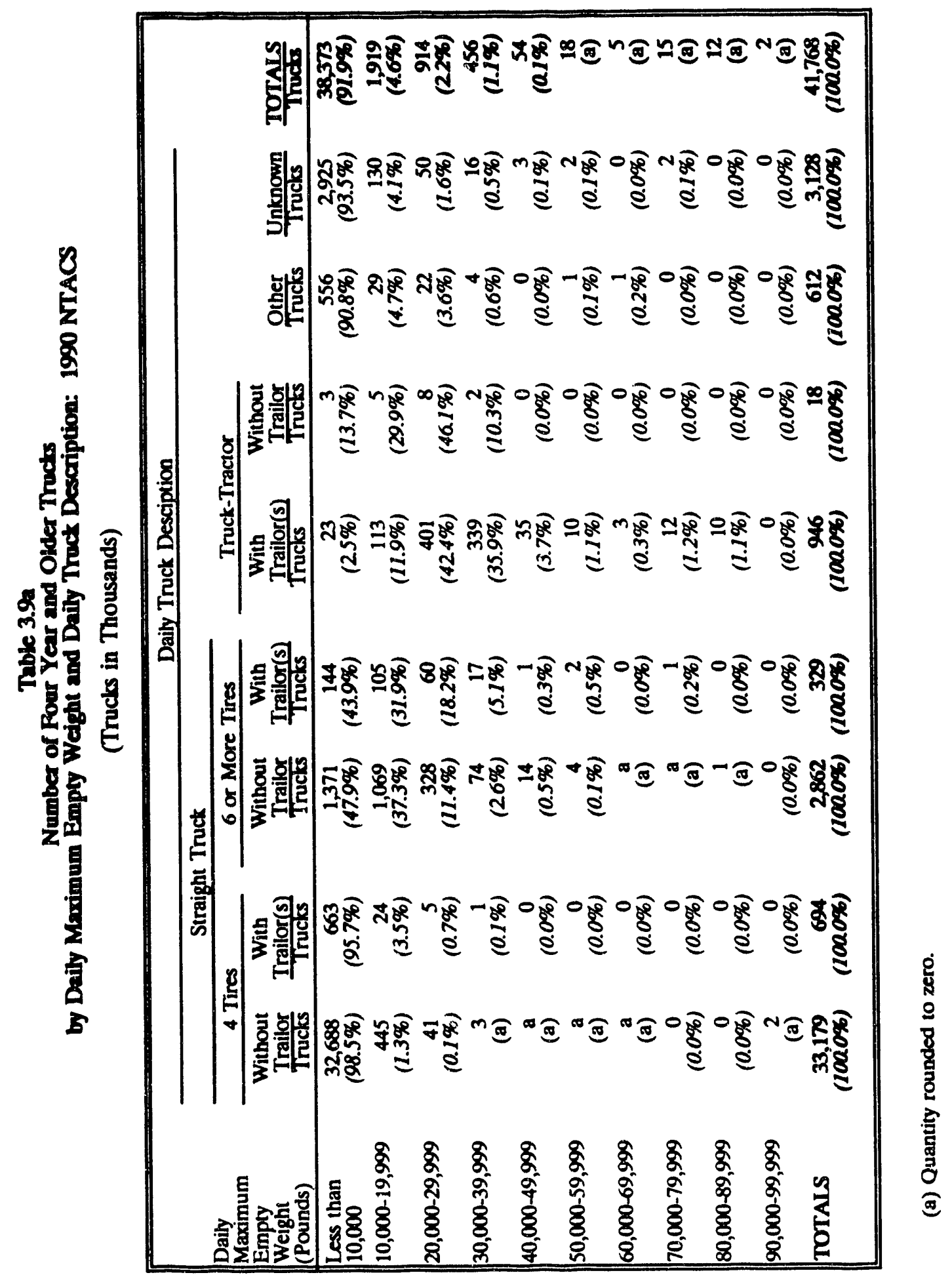




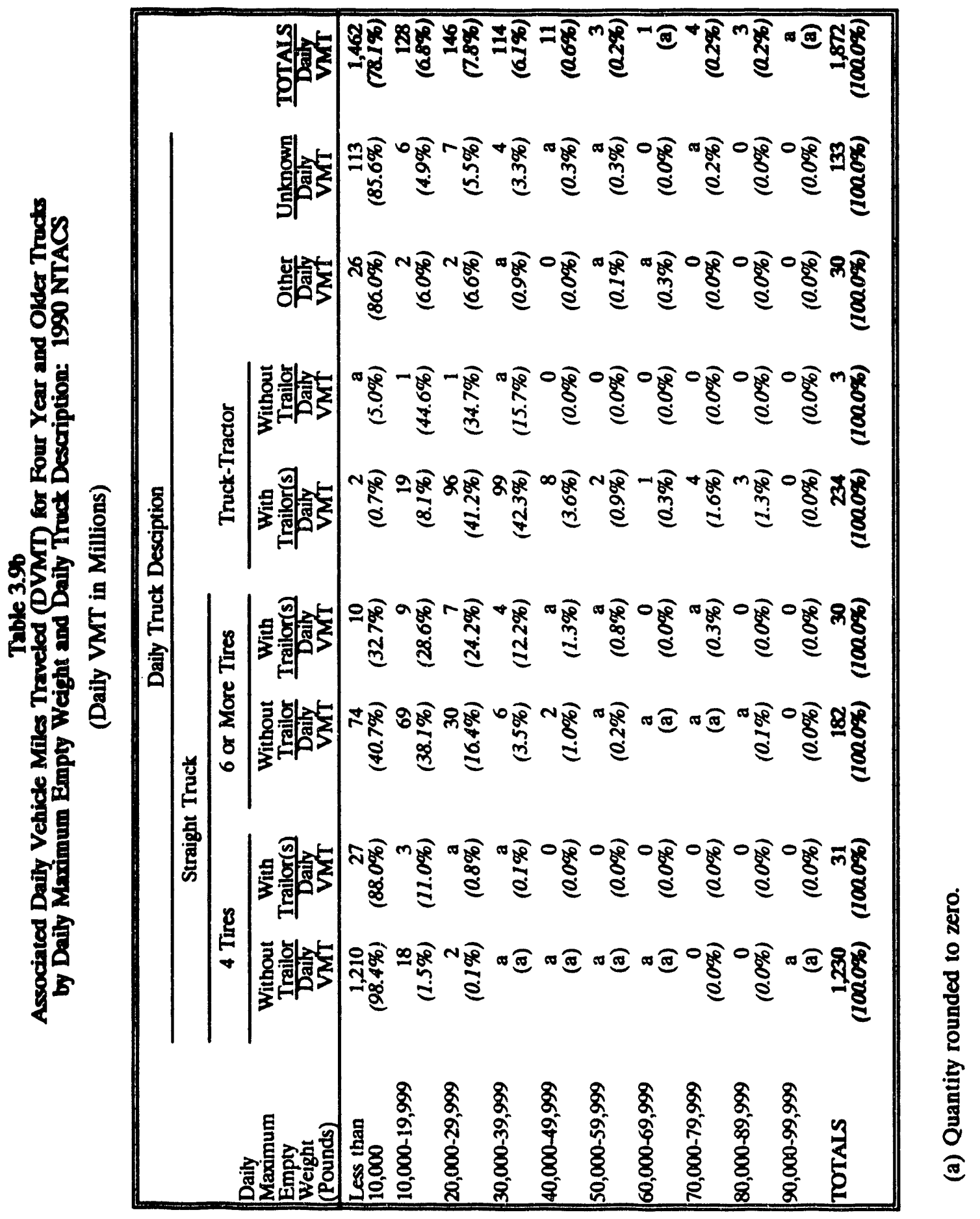




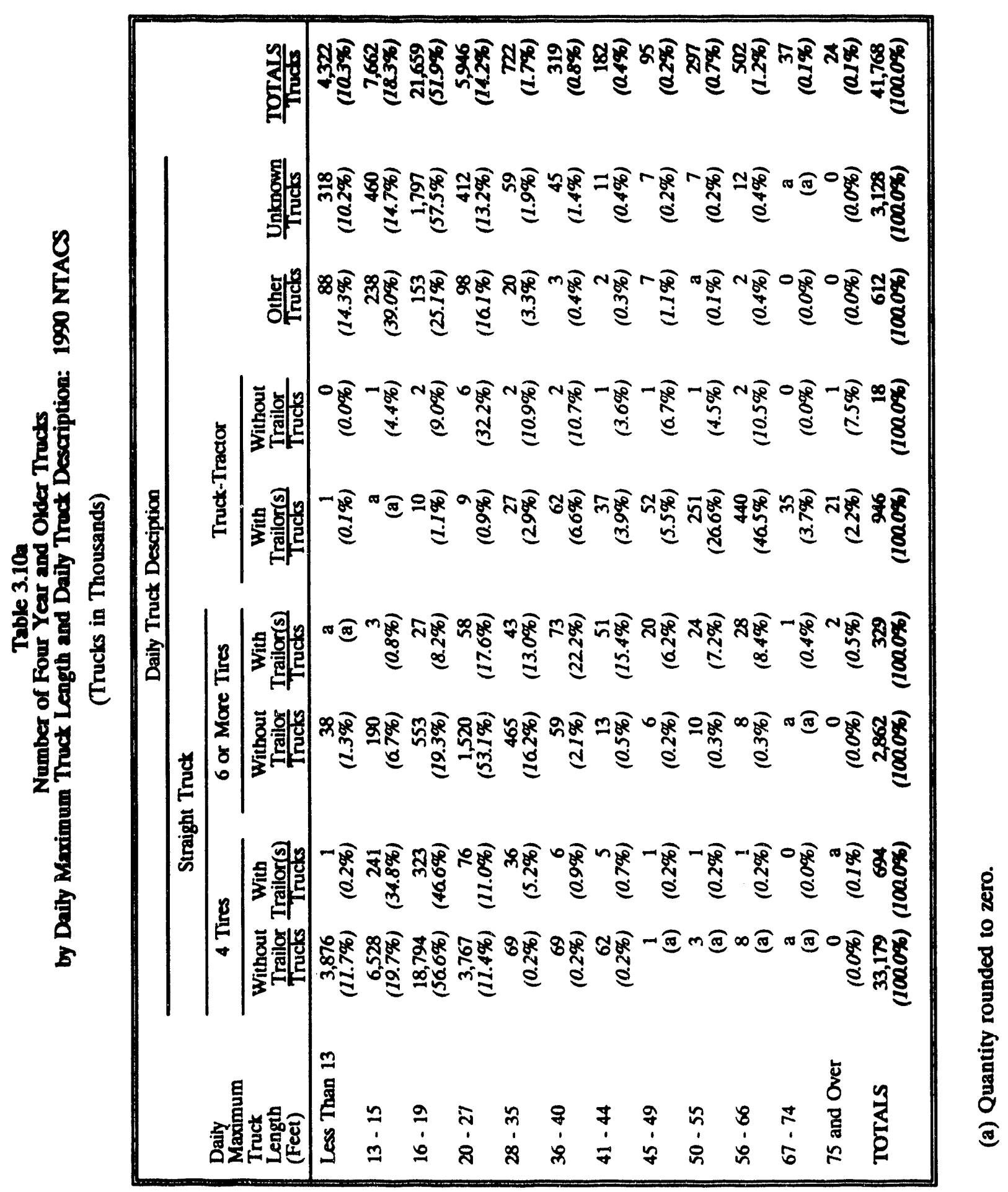




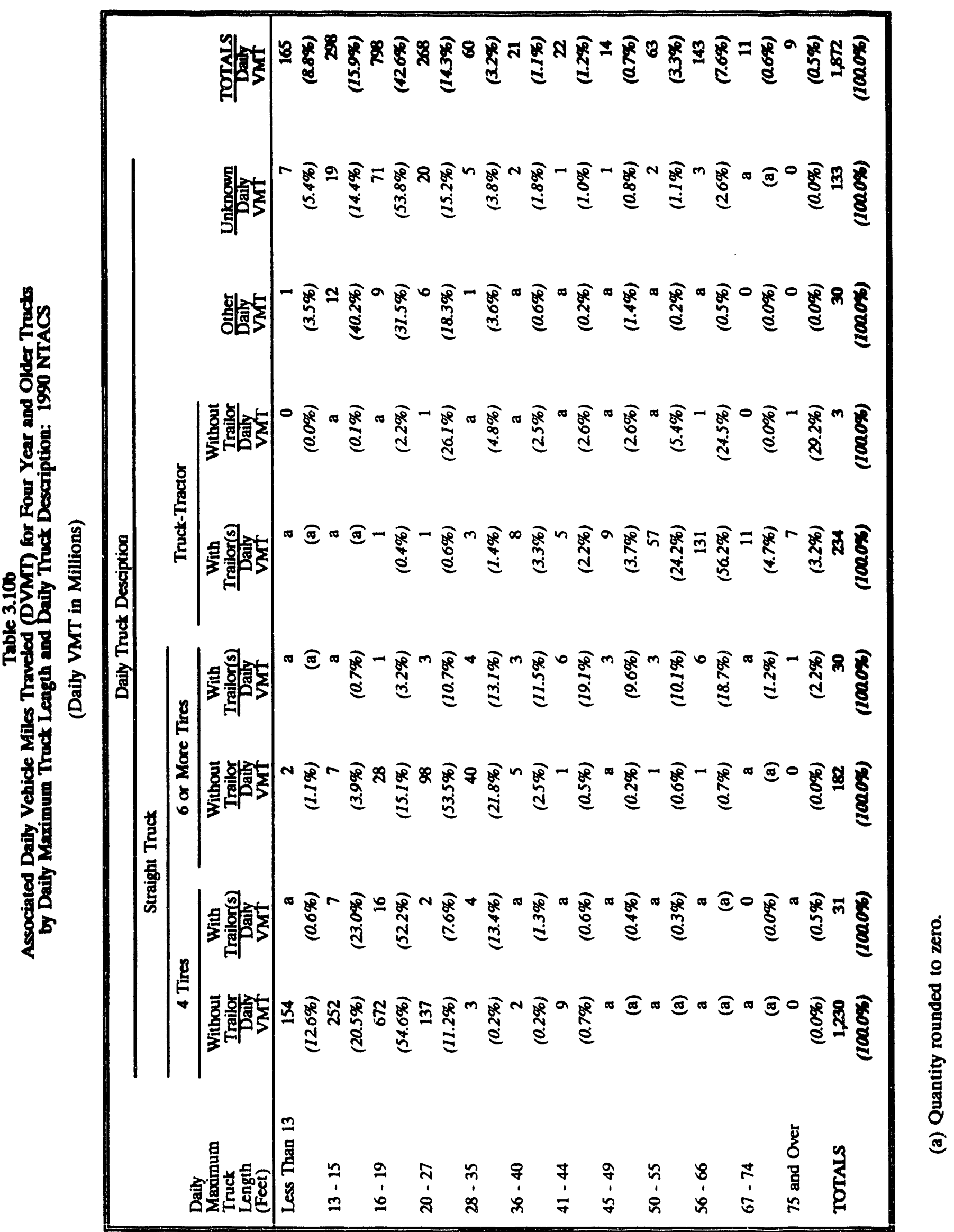




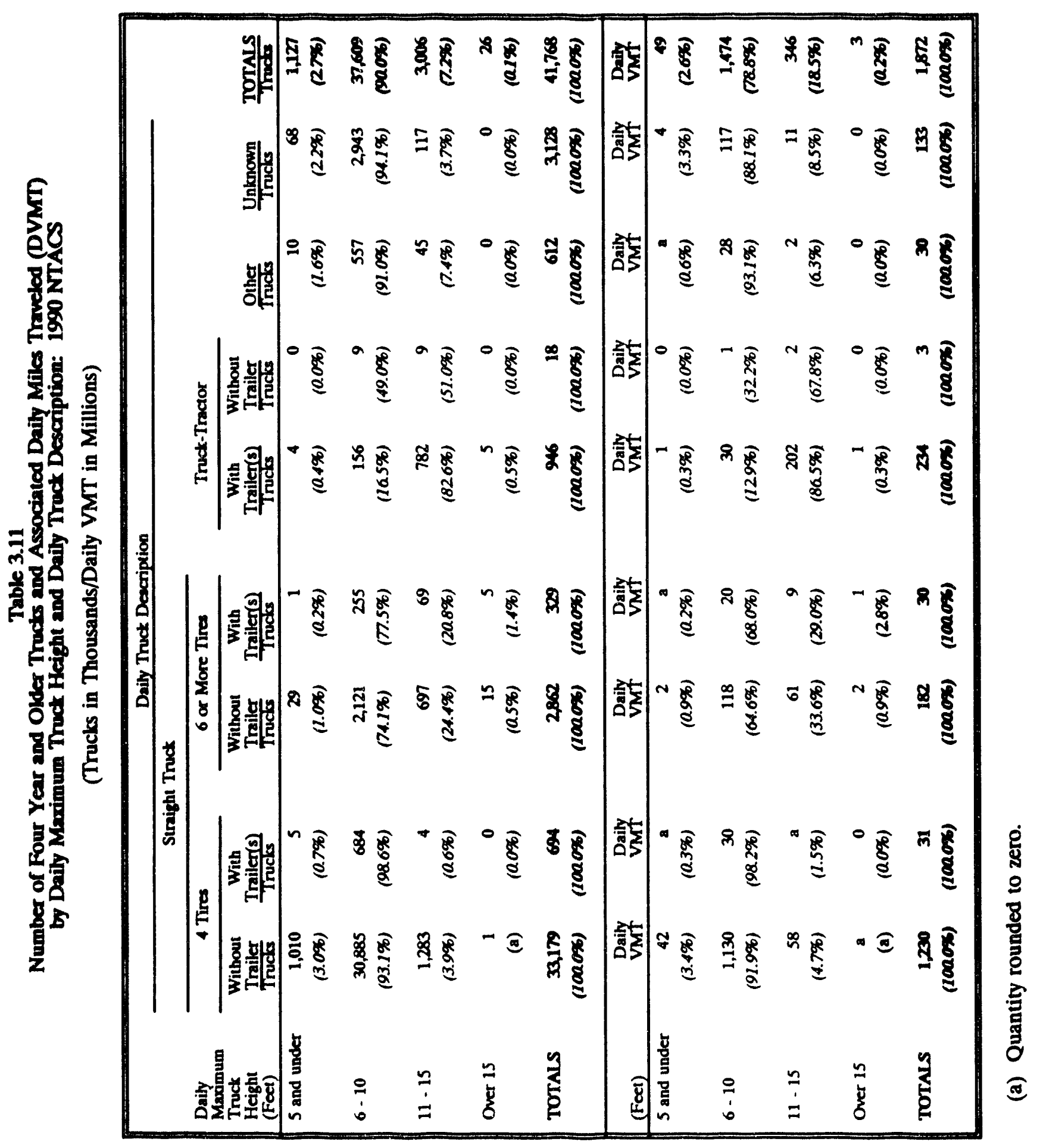




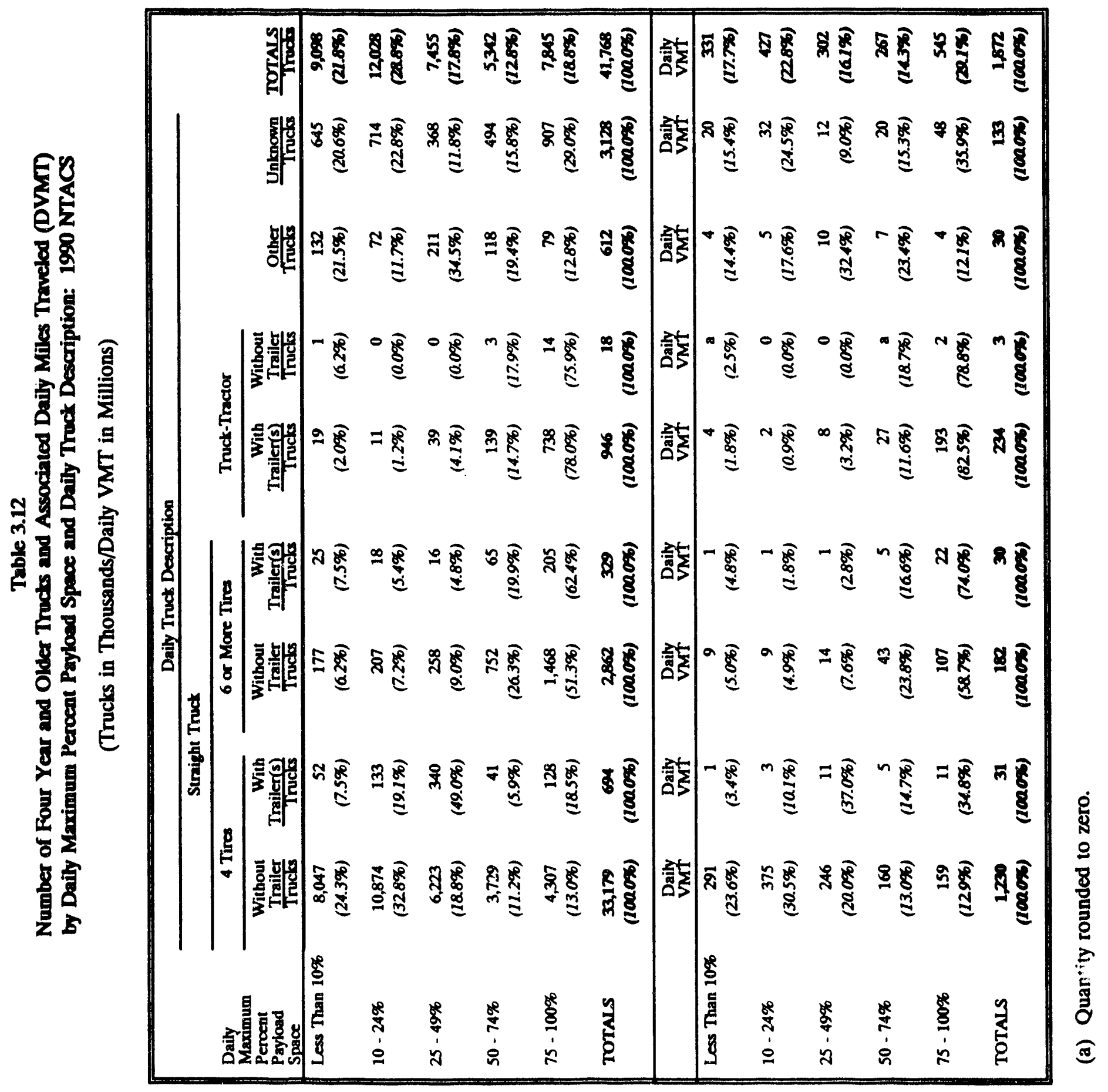




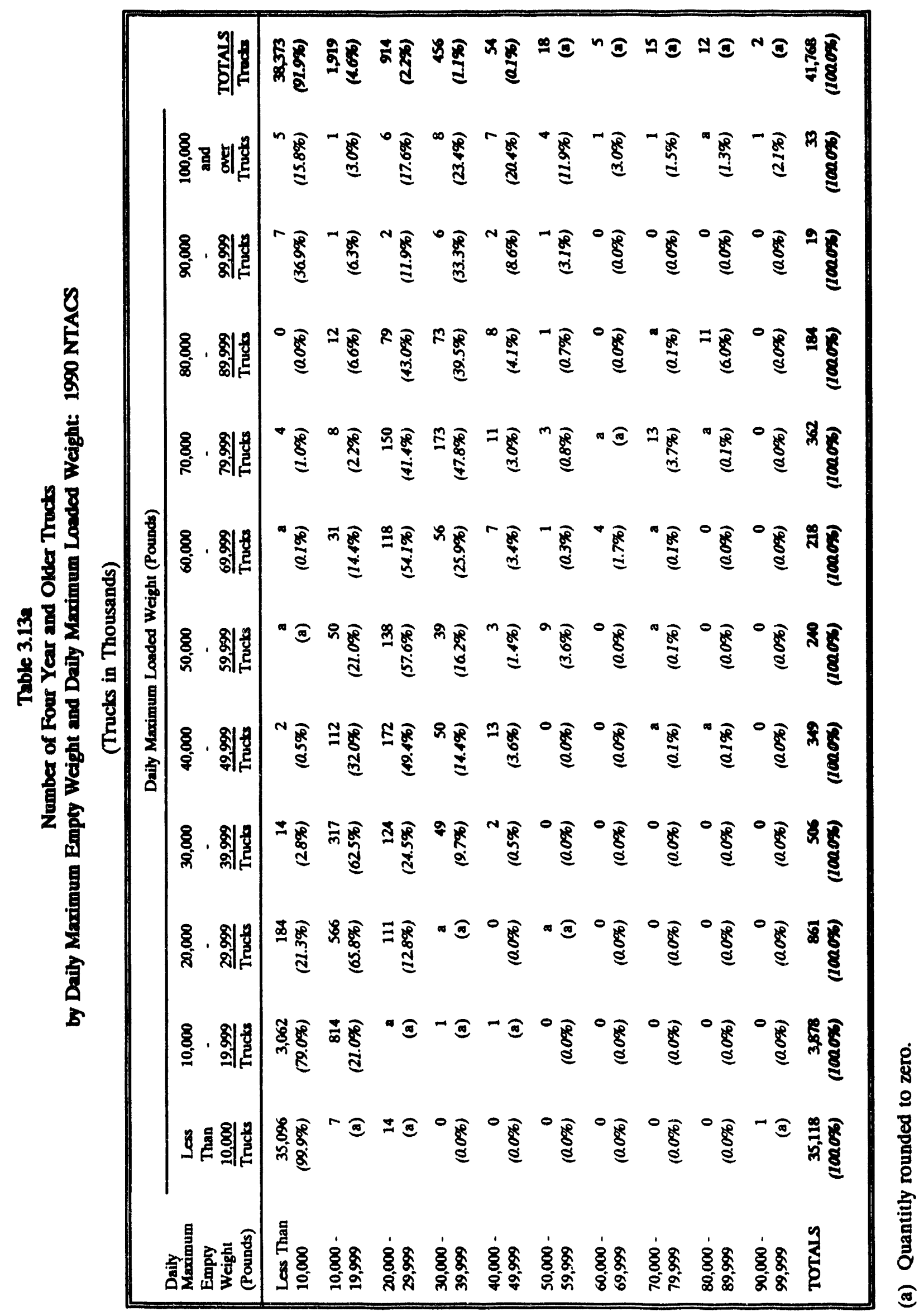




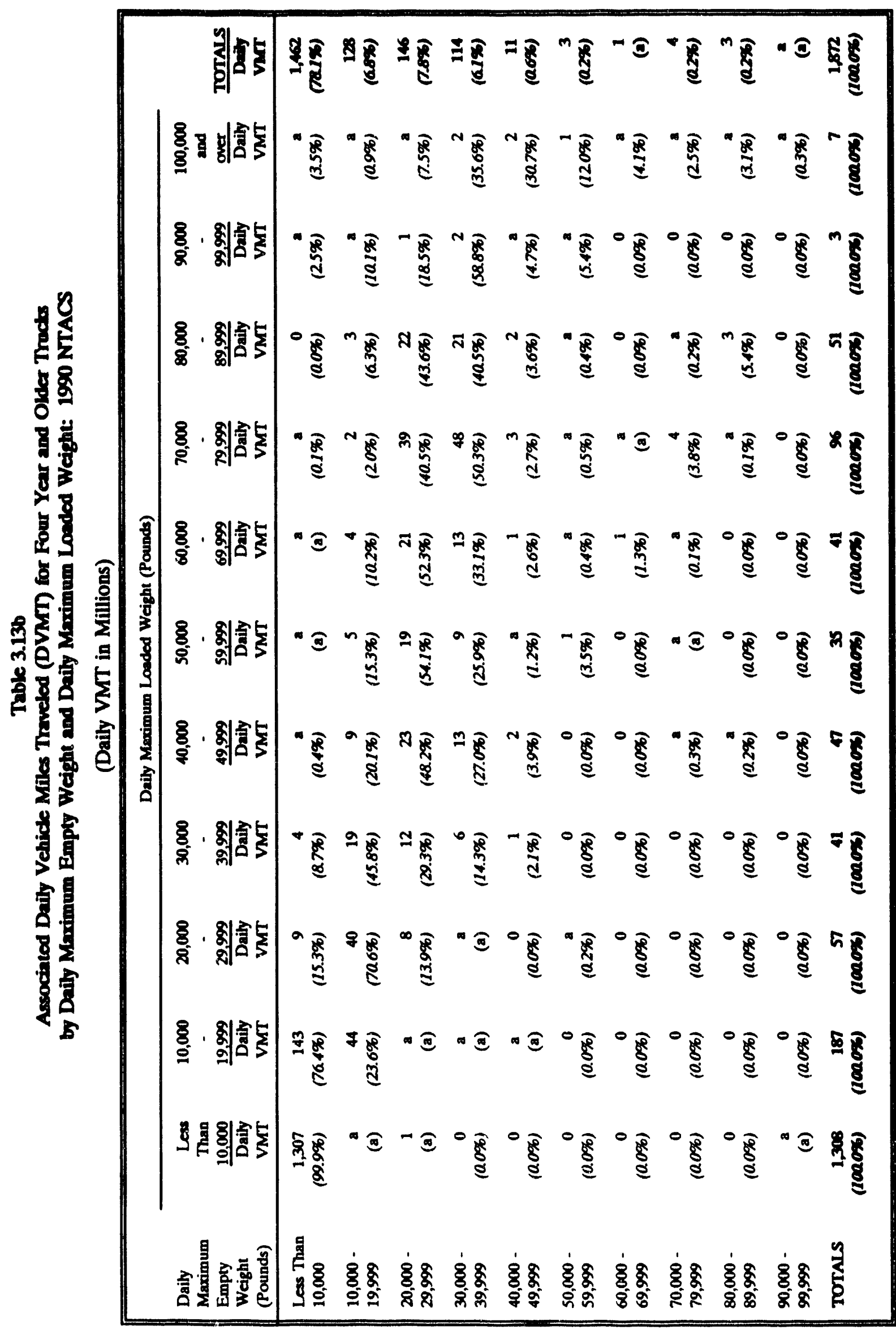

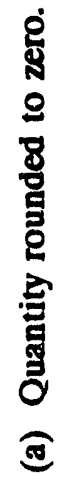




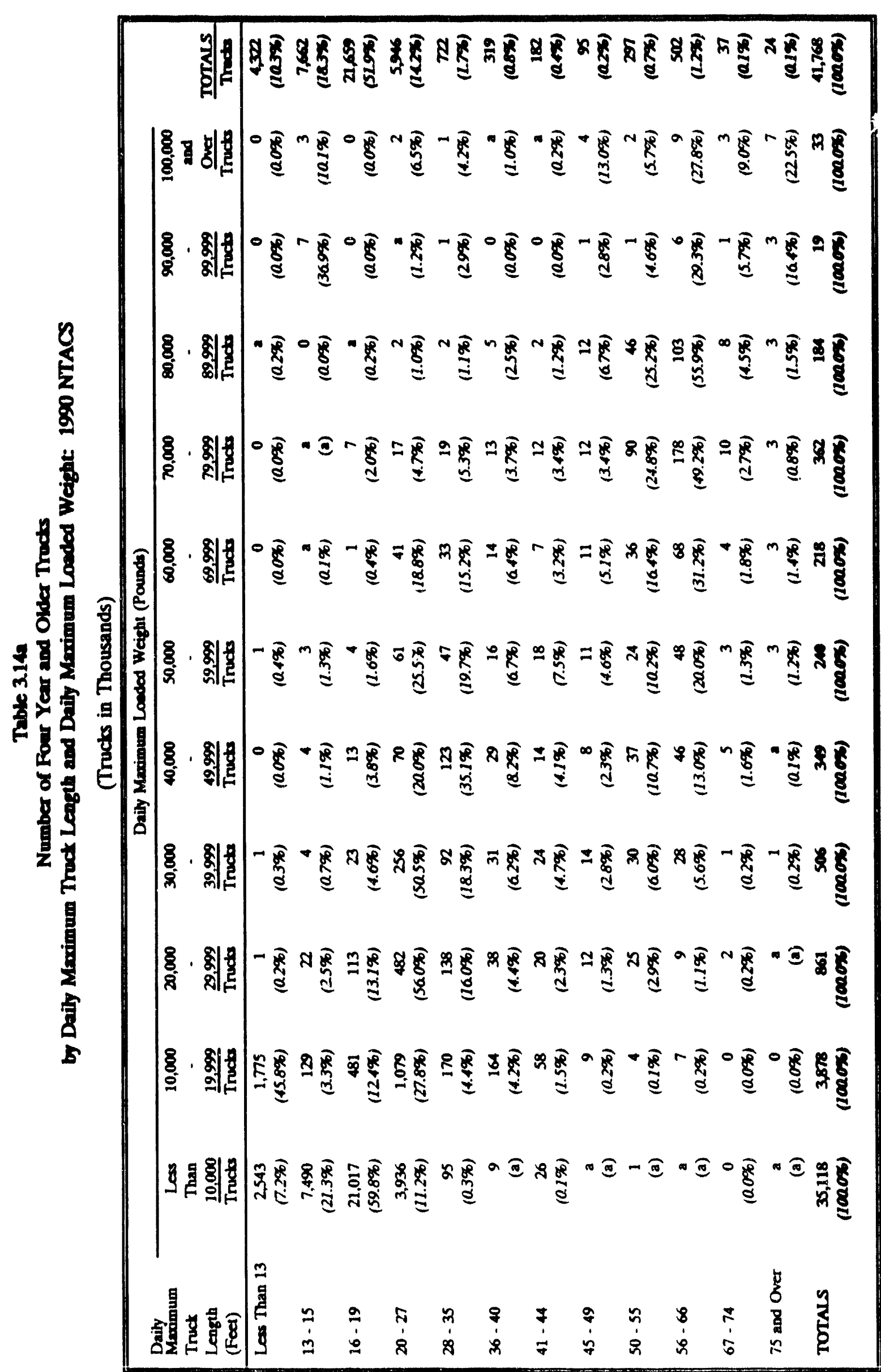

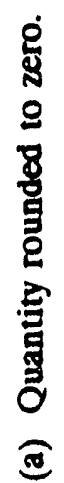




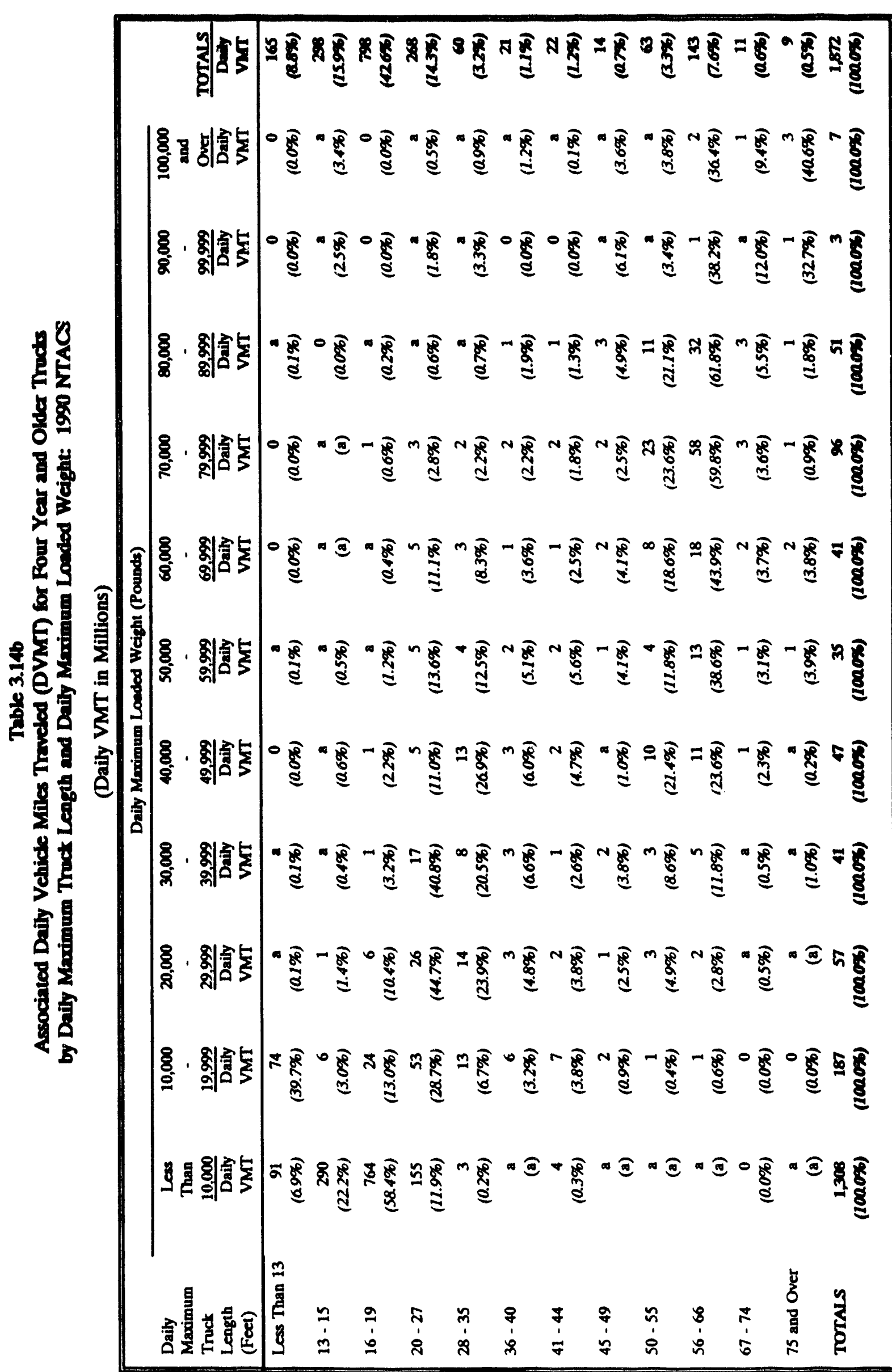




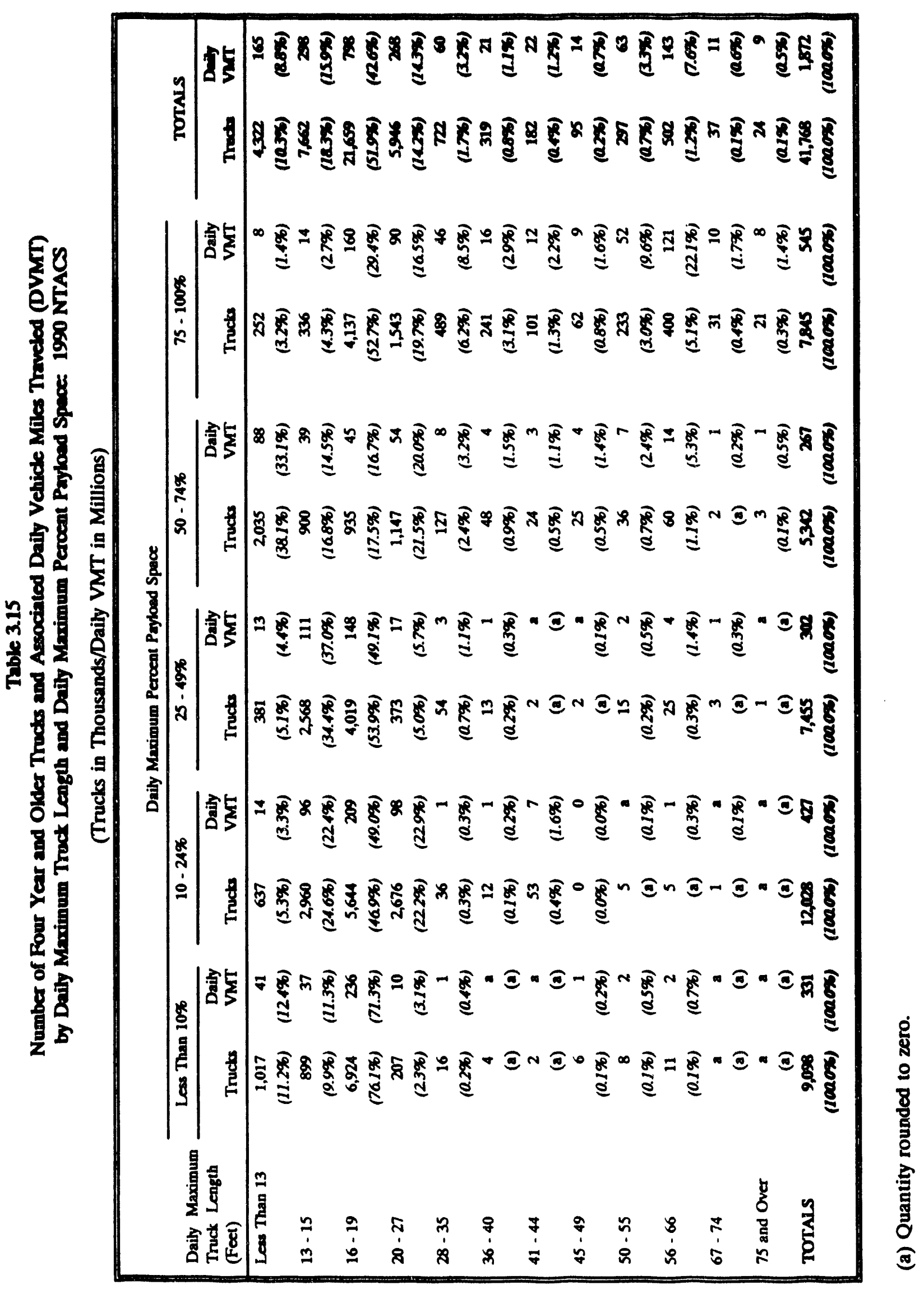


Table 3.16

Number of Four Year and Older Trucks and Associated Daily Vehicle Miles Traveled (DVMT) by Daily Commodity Carried: 1990 NTACS

(Trucks in Thousands/Daily VMT in Millions)

\begin{tabular}{|c|c|c|c|c|}
\hline Daily Commodity Carried & \multicolumn{2}{|c|}{ Trucks } & \multicolumn{2}{|c|}{ Daily VMT } \\
\hline \multicolumn{5}{|l|}{ Agricultural and Food Products } \\
\hline - Live Animaks & 158 & $(0.4 \%)$ & 6 & $(0.3 \%)$ \\
\hline - Fresh Farm Products & 261 & $(0.6 \%)$ & 14 & $(0.7 \%)$ \\
\hline - Processed Foods & 779 & $(1.9 \%)$ & 63 & $(3.4 \%)$ \\
\hline Mining Products, Unrefined - Crude Oil, Coal, and Metal Ores ...... & 109 & (0.3\%) & 8 & $(0.4 \%)$ \\
\hline Building Materials - Gravel, Sand, Concrete, Glass, and Stone, etc. . . . & 180 & $(0.4 \%)$ & 17 & $(0.9 \%)$ \\
\hline \multicolumn{5}{|l|}{ Forestry, Wood, and Paper Products } \\
\hline - Logs and Forest Products . . . . . . . . . & 61 & $(0.1 \%)$ & 6 & $(0.3 \%)$ \\
\hline - Lumber and Fabricated Wood Products - Except Furniture . . & 125 & $(0.3 \%)$ & 11 & $(0.6 \%)$ \\
\hline - Paper, Princed Matter, and Paper Products & 99 & $(0.2 \%)$ & 13 & $(0.7 \%)$ \\
\hline \multicolumn{5}{|l|}{ Chemicals, Petroleum, and Allied Products } \\
\hline - Chemicals and/or Drugs & 330 & $(0.8 \%)$ & 25 & $(1.4 \%)$ \\
\hline - Petroleum, Petroleum Products, Paving, and Asphalt or Tar Cements & 518 & $(1.2 \%)$ & 32 & (1.7\%) \\
\hline - Plastics and/or Rubber Products & 215 & (0.5\%) & 32 & $(1.7 \%)$ \\
\hline \multicolumn{5}{|l|}{ Metats and Metal Products } \\
\hline - Primary Metal Products & 642 & (1.5\%) & 38 & $(20 \%)$ \\
\hline - Fabricated Metal Products and Bolts and Nuts & 425 & $(1.0 \%)$ & 37 & $(20 \%)$ \\
\hline - Machinery & 330 & $(0.8 \%)$ & 39 & $(21 \%)$ \\
\hline - Transportation Equipment and Parts ......... & 598 & $(1.4 \%)$ & 36 & $(1.9 \%)$ \\
\hline \multicolumn{5}{|l|}{ Other Manufactured Products } \\
\hline - Furniture (Wood and Non-wood) and/or Fixtures & 286 & $(0.7 \%)$ & 24 & $(1.3 \%)$ \\
\hline - Textiles and Apparels & 410 & $(1.0 \%)$ & 37 & $(20 \%)$ \\
\hline - Miscellaneous Products of Manufacturing & 298 & $(0.7 \%)$ & 24 & (1.3\%) \\
\hline \multicolumn{5}{|l|}{ Miscellaneous } \\
\hline - Moving of Household and Office Furniture, Including Exhibits .... & 198 & $(0.5 \%)$ & 16 & $(0.9 \%)$ \\
\hline - Mixed Cargo, General Freight, Mail and Express Traffic, etc. .... & 990 & $(24 \%)$ & 63 & $(3.4 \%)$ \\
\hline - Tools/Parts for Specialized Use as in Craftsman's Vehicle & 2,165 & $(5.2 \%)$ & 82 & (4.4\%) \\
\hline - Scrap, Garbage, Trash & 1,383 & $(3.3 \%)$ & 49 & $(26 \%)$ \\
\hline - Industrial Water ... & 314 & $(0.8 \%)$ & 12 & $(0.7 \%)$ \\
\hline Other $\ldots \ldots \ldots \ldots \ldots$ & 21,204 & $(50.8 \%)$ & 745 & $(39.7 \%)$ \\
\hline No Load Carried/Vehicle Empty & 9,690 & $(23.2 \%)$ & 440 & (23.5\%) \\
\hline TOTALS & 41,768 & $(100.0 \%)$ & 1,872 & (1000\%) \\
\hline
\end{tabular}


Table 3.17

Number of Four lear and Older Trucks and Associated Daily Vehicle Miles Traveled (DVMT) by Daily Major Use and Daily Hazardous Material Carrying Status: 1990 NTACS

(Trucks in Thousands/Daily VMT in Millions)

\begin{tabular}{|c|c|c|c|c|c|c|c|c|}
\hline \multirow{3}{*}{$\begin{array}{l}\text { Daily } \\
\text { Major } \\
\text { Use }\end{array}$} & \multicolumn{6}{|c|}{ Daily Hazardous Material Carrying Status } & & \\
\hline & \multicolumn{2}{|c|}{ Yes } & \multicolumn{2}{|c|}{ No } & \multicolumn{2}{|c|}{ Unknown } & \multicolumn{2}{|c|}{ TOTALS } \\
\hline & Trucks & $\begin{array}{l}\text { Daily } \\
\text { VMT }\end{array}$ & Trucks & $\begin{array}{l}\text { Daily } \\
\text { VMT }\end{array}$ & Trucks & $\begin{array}{l}\text { Daily } \\
\text { VMT }\end{array}$ & Tructs & $\begin{array}{l}\text { Daily } \\
\text { VMT }\end{array}$ \\
\hline $\begin{array}{l}\text { Personal } \\
\text { Transportation }\end{array}$ & $(0.6 \%)$ & $\begin{array}{l}a \\
\text { (a) }\end{array}$ & $\begin{array}{r}1,898 \\
(18.3 \%)\end{array}$ & $\begin{array}{r}72 \\
(9.8 \%)\end{array}$ & $\begin{array}{c}27,862 \\
(89.2 \%)\end{array}$ & $\begin{array}{c}950 \\
(85.7 \%)\end{array}$ & $\begin{array}{c}29,760 \\
(71.3 \%)\end{array}$ & $\begin{array}{c}1,022 \\
(54.6 \%)\end{array}$ \\
\hline $\begin{array}{l}\text { Contract } \\
\text { Carrier }\end{array}$ & (13.2\%) & $\begin{array}{c}5 \\
(19.6 \%)\end{array}$ & $\begin{array}{c}518 \\
(5.0 \%)\end{array}$ & $\begin{array}{r}83 \\
(11.3 \%)\end{array}$ & $\begin{array}{c}103 \\
(0.3 \%)\end{array}$ & $\begin{array}{c}5 \\
(0.4 \%)\end{array}$ & $\begin{array}{c}645 \\
(1.5 \%)\end{array}$ & $\begin{array}{c}93 \\
(5.0 \%)\end{array}$ \\
\hline $\begin{array}{l}\text { Common } \\
\text { Carrier }\end{array}$ & $\begin{array}{c}51 \\
(27.0 \%)\end{array}$ & $\begin{array}{c}9 \\
(34.8 \%)\end{array}$ & $\begin{array}{c}1,220 \\
(11.8 \%)\end{array}$ & $\begin{array}{c}167 \\
(227 \%)\end{array}$ & $(0.1 \%)$ & $(0.3 \%)$ & $\begin{array}{l}1,314 \\
(3,1 \%)\end{array}$ & $\begin{array}{c}180 \\
(9.6 \%)\end{array}$ \\
\hline $\begin{array}{l}\text { Other } \\
\text { Business Use }\end{array}$ & $\begin{array}{c}103 \\
(55.0 \%)\end{array}$ & (43.3\%) & $\begin{array}{c}6,073 \\
(58.7 \%)\end{array}$ & $\begin{array}{c}379 \\
(51.4 \%)\end{array}$ & $\begin{array}{c}820 \\
(26 \%)\end{array}$ & $\begin{array}{c}53 \\
(4.8 \%)\end{array}$ & $\begin{array}{c}6,996 \\
(168 \%)\end{array}$ & $\begin{array}{c}443 \\
(23.6 \%)\end{array}$ \\
\hline Unknown & $\begin{array}{c}8 \\
(4.2 \%)\end{array}$ & (23\%) & $\begin{array}{c}633 \\
(a .1 \%)\end{array}$ & $\begin{array}{c}36 \\
(4.9 \%)\end{array}$ & $\begin{array}{c}2,410 \\
(7.7 \%)\end{array}$ & $\begin{array}{c}98 \\
(8.8 \%)\end{array}$ & $\begin{array}{c}3,051 \\
(7.3 \%)\end{array}$ & $\begin{array}{c}135 \\
(7.2 \%)\end{array}$ \\
\hline TOTALS & $\begin{array}{c}187 \\
(1000 \%)\end{array}$ & $\begin{array}{c}26 \\
(1000 \%)\end{array}$ & $\begin{array}{c}10,342 \\
(1000 \%)\end{array}$ & $\begin{array}{c}737 \\
(1000 \%)\end{array}$ & $\begin{array}{c}31,239 \\
(100.0 \%)\end{array}$ & $\begin{array}{c}1,109 \\
(100 \% 0 \%)\end{array}$ & $\begin{array}{c}41,768 \\
(1000 \%)\end{array}$ & $\begin{array}{c}1,872 \\
(1000 \%)\end{array}$ \\
\hline
\end{tabular}

(a) Quantity rounded to zero. 
Table 3.18

Number of Four Year and Older Trucks and Associated Daily Vehicle Miles Traveled (DVMT) by Daily Truck Description and Daily Hazardous Material Carrying Status: 1990 NTACS

(Trucks in Thousands/Daily VMT in Millions)

\begin{tabular}{|c|c|c|c|c|c|c|c|c|}
\hline \multirow{3}{*}{$\begin{array}{l}\text { Daily } \\
\text { Truck } \\
\text { Description }\end{array}$} & \multicolumn{6}{|c|}{ Daily Hazardous Material Carrying Status } & & \\
\hline & \multicolumn{2}{|c|}{ Yes } & \multicolumn{2}{|c|}{ No } & \multicolumn{2}{|c|}{ Unknown } & \multicolumn{2}{|c|}{ TOTALS } \\
\hline & Trucks & $\begin{array}{l}\text { Daily } \\
\text { VMT }\end{array}$ & Trucks & $\begin{array}{l}\text { Daily } \\
\text { VMT }\end{array}$ & Trucks & $\begin{array}{l}\text { Daity } \\
\text { VMT }\end{array}$ & Tructer & $\begin{array}{l}\text { Daily } \\
\text { vMt }\end{array}$ \\
\hline \multicolumn{9}{|l|}{ Straight Truck } \\
\hline $\begin{array}{l}\text { - With } 4 \text { Tires } \\
\text { Without Trailer }\end{array}$ & $\begin{array}{c}8 \\
(4.3 \%)\end{array}$ & $(28 \%)$ & $\begin{array}{c}5,922 \\
(57.3 \%)\end{array}$ & $\begin{array}{c}290 \\
(39.3 \%)\end{array}$ & $\begin{array}{c}27,250 \\
(87.2 \%)\end{array}$ & $\begin{array}{r}940 \\
(84.7 \%)\end{array}$ & $\begin{array}{l}33,179 \\
(79,4 \pi)\end{array}$ & $\begin{array}{l}1,230 \\
(65.7 \%)\end{array}$ \\
\hline $\begin{array}{l}\text { - With } 4 \text { Tirea } \\
\text { With Trailer(s) }\end{array}$ & (a) & (a) & $\begin{array}{c}150 \\
(1.5 \%)\end{array}$ & $\begin{array}{c}8 \\
(1.1 \%)\end{array}$ & $\begin{array}{c}543 \\
(1.7 \%)\end{array}$ & $\stackrel{23}{(20 \%)}$ & $(1.7 \%)$ & $\begin{array}{c}31 \\
(1.6 \%)\end{array}$ \\
\hline $\begin{array}{l}\text { - With } 6 \text { or More Tires } \\
\text { Without Trailer }\end{array}$ & $\begin{array}{c}102 \\
(54.4 \%)\end{array}$ & $\begin{array}{c}8 \\
(29.3 \%)\end{array}$ & $\begin{array}{c}2,332 \\
(226 \%)\end{array}$ & $\begin{array}{c}155 \\
(21.0 \%)\end{array}$ & $\begin{array}{c}428 \\
(1.4 \%)\end{array}$ & $\begin{array}{c}20 \\
(1.8 \%)\end{array}$ & $\begin{array}{l}2860 \\
(69 \%)\end{array}$ & $(9.7 \%)$ \\
\hline $\begin{array}{l}\text { - With } 6 \text { or More Tires } \\
\text { With Trailer(s) }\end{array}$ & $(21 \%)$ & $(3.5 \%)$ & $\begin{array}{c}295 \\
(29 \%)\end{array}$ & $\begin{array}{c}27 \\
(3.7 \%)\end{array}$ & $(0.1 \%)$ & $(0.2 \%)$ & (a8\%) & $\begin{array}{c}30 \\
(1.6 \%)\end{array}$ \\
\hline \multicolumn{9}{|l|}{ Truck-Tractor } \\
\hline - With Trailer(s) & $\begin{array}{r}62 \\
(33.2 \%)\end{array}$ & $\begin{array}{c}15 \\
(59.7 \%)\end{array}$ & $\begin{array}{c}838 \\
(8.1 \%)\end{array}$ & $\begin{array}{c}210 \\
(28.5 \%)\end{array}$ & $\begin{array}{c}45 \\
(0.1 \%)\end{array}$ & $\begin{array}{c}8 \\
(0.7 \%)\end{array}$ & (2.3\%) & (125\%) \\
\hline - Without Trailer & $(0.2 \%)$ & $(0.1 \%)$ & $(0.2 \%)$ & $(0.3 \%)$ & $\begin{array}{l}3 \\
(a)\end{array}$ & (a) & $\begin{array}{l}18 \\
(x)\end{array}$ & $\left(a_{1}^{3}\right)$ \\
\hline Other & $(2.3 \%)$ & $\frac{1}{(24 \%)}$ & $\begin{array}{c}198 \\
(1.9 \%)\end{array}$ & $\begin{array}{c}13 \\
(1.8 \%)\end{array}$ & $\begin{array}{c}410 \\
(1.3 \%)\end{array}$ & $\stackrel{16}{(1.5 \%)}$ & $\begin{array}{c}612 \\
(2.5 \%)\end{array}$ & $\begin{array}{r}30 \\
(1.6 \%)\end{array}$ \\
\hline Unknown & $(3.5 \%)$ & $\frac{1}{(2.3 \%)}$ & $\begin{array}{c}591 \\
(5.7 \%)\end{array}$ & $\begin{array}{c}32 \\
(4.4 \%)\end{array}$ & $\begin{array}{c}2,530 \\
(8.1 \%)\end{array}$ & $\begin{array}{c}100 \\
(9.0 \%)\end{array}$ & $\begin{array}{c}3,128 \\
(7.5 \%)\end{array}$ & (7.1\%) \\
\hline TOTALS & $\begin{array}{c}187 \\
(1000 \%)\end{array}$ & $(1000 \%)$ & $\begin{array}{c}10,342 \\
(1000 \%)\end{array}$ & $\begin{array}{r}737 \\
(1000 \%)\end{array}$ & $\begin{array}{c}31,239 \\
(1000 \%)\end{array}$ & $\begin{array}{c}1,109 \\
(1000 \%)\end{array}$ & $\begin{array}{c}41,763 \\
(1020 \% 5)\end{array}$ & $\begin{array}{c}1,872 \\
(1000 \%)\end{array}$ \\
\hline
\end{tabular}

(a) Quantity rounded to zero. 
Table 3.19

Number of Four Year and Older Tructs and Associated Daily Vehicle Miles Traveled (DVMT) by Daily Percent Interstate, Daily Percent Four Lane, Daily Percent Off Road and Daily Hazardous Material Carrying Status: 1990 NTACS

(Trucks in Thousands/Daily VMT in Millions)

\begin{tabular}{|c|c|c|c|c|c|c|c|c|}
\hline & \multicolumn{6}{|c|}{ Daily Hazardous Material Carrying Status } & & \\
\hline & \multicolumn{2}{|c|}{ Yes } & \multicolumn{2}{|c|}{ No } & \multicolumn{2}{|c|}{ Unknown } & \multicolumn{2}{|c|}{ TOTALS } \\
\hline & Trucke & $\begin{array}{l}\text { Daily } \\
\text { VMT } \\
\end{array}$ & Trucks & $\begin{array}{l}\text { Daity } \\
\text { VMT }\end{array}$ & Trucks & $\begin{array}{l}\text { Daily } \\
\text { VMT } \\
\end{array}$ & Tructs & $\begin{array}{l}\text { Daily } \\
\text { VMT }\end{array}$ \\
\hline \multicolumn{9}{|c|}{ Daily Percent Interutate } \\
\hline Under $10 \%$ & $\begin{array}{c}102 \\
(54.3 \%)\end{array}$ & $(28.8 \%)$ & $\begin{array}{c}6,007 \\
(58.1 \%)\end{array}$ & $\begin{array}{c}268 \\
(36.3 \%)\end{array}$ & $\begin{array}{l}15,927 \\
(51.0 \%)\end{array}$ & $\begin{array}{c}403 \\
(36.4 \%)\end{array}$ & $\begin{array}{c}22,035 \\
(528 \%)\end{array}$ & $\begin{array}{c}679 \\
(36.3 \%)\end{array}$ \\
\hline $10 \cdot 24 \%$ & $\begin{array}{c}15 \\
(7.8 \%)\end{array}$ & $(14.7 \%)$ & $\begin{array}{c}875 \\
(8.5 \%)\end{array}$ & $\begin{array}{c}67 \\
(9.1 \%)\end{array}$ & $\begin{array}{c}1,566 \\
(5.0 \%)\end{array}$ & $\begin{array}{c}74 \\
(0.7 \%)\end{array}$ & $\begin{array}{c}2,455 \\
(5.9 \%)\end{array}$ & $(7.7 \%)$ \\
\hline $25-49 \%$ & $\begin{array}{c}19 \\
(10.1 \%)\end{array}$ & $\left(11.1 \%^{3}\right)$ & $\begin{array}{c}441 \\
(4.3 \%)\end{array}$ & $\begin{array}{c}49 \\
(0.7 \%)\end{array}$ & $\begin{array}{c}1,593 \\
(5.1 \%)\end{array}$ & $\begin{array}{c}100 \\
(9.1 \%)\end{array}$ & $\begin{array}{c}2,053 \\
(2,9 \%)\end{array}$ & $\begin{array}{c}153 \\
(8.1 \%)\end{array}$ \\
\hline $50-74 \%$ & $(6.2 \%)$ & $(9.2 \%)$ & $\begin{array}{c}964 \\
(9.3 \%)\end{array}$ & $\begin{array}{c}109 \\
(14.7 \%)\end{array}$ & $\begin{array}{c}1,909 \\
\text { (6.1\%) }\end{array}$ & $\begin{array}{c}117 \\
(10.5 \%)\end{array}$ & $\begin{array}{l}2885 \\
(69 \%)\end{array}$ & (122\%) \\
\hline $75-100 \%$ & $\begin{array}{c}37 \\
(19.9 \%)\end{array}$ & (34.6\%) & $\begin{array}{c}1,252 \\
(121 \%)\end{array}$ & $\begin{array}{c}207 \\
(28.0 \%)\end{array}$ & $\begin{array}{c}3,307 \\
(10.6 \%)\end{array}$ & $\begin{array}{c}163 \\
(14.7 \%)\end{array}$ & $\begin{array}{r}4596 \\
(11.0 \%)\end{array}$ & $\begin{array}{c}379 \\
(202 \%)\end{array}$ \\
\hline Unknown & $(1.6 \%)$ & $(1.7 \%)$ & $\begin{array}{c}804 \\
(7.8 \%)\end{array}$ & $\begin{array}{c}38 \\
(5.2 \%)\end{array}$ & $\begin{array}{c}6,937 \\
(222 \%)\end{array}$ & $\begin{array}{c}251 \\
(22.6 \%)\end{array}$ & $\begin{array}{c}7,743 \\
(1 \& 5 \%)\end{array}$ & $\begin{array}{r}289 \\
(155 \%)\end{array}$ \\
\hline TOTALS & $\frac{187}{(1000 \%)}$ & $(1000 \%)$ & $\begin{array}{c}10,342 \\
(1000 \%)\end{array}$ & $\begin{array}{c}737 \\
(1000 \%)\end{array}$ & $\begin{array}{c}31,239 \\
(1000 \%)\end{array}$ & $\begin{array}{c}1,109 \\
(100.0 \%)\end{array}$ & $\begin{array}{c}41,768 \\
(1000 \%)\end{array}$ & $\begin{array}{c}1.872 \\
(1020 \%)\end{array}$ \\
\hline \multicolumn{9}{|c|}{ Daily Percent Four Lane } \\
\hline Under $10 \%$ & $\begin{array}{c}114 \\
(60.7 \%)\end{array}$ & $(53.0 \%)$ & $\begin{array}{c}4,999 \\
(48.3 \%)\end{array}$ & $\begin{array}{c}341 \\
(46.3 \%)\end{array}$ & $\begin{array}{c}15,367 \\
(49.2 \%)\end{array}$ & $\begin{array}{c}470 \\
(42.4 \%)\end{array}$ & $\begin{array}{c}20,480 \\
(49.0 \%)\end{array}$ & (44.1\%) \\
\hline $10-24 \%$ & $(127 \%)$ & $(18.1 \%)$ & $\begin{array}{c}1,741 \\
(168 \%)\end{array}$ & $\begin{array}{c}151 \\
(20.4 \%)\end{array}$ & $\begin{array}{c}2,316 \\
(7.4 \%)\end{array}$ & $\begin{array}{c}109 \\
(9.8 \%)\end{array}$ & $\begin{array}{c}4.081 \\
(9.8 \% 5)\end{array}$ & $\begin{array}{c}265 \\
(14.1 \%)\end{array}$ \\
\hline $25 \cdot 49 \%$ & $\begin{array}{c}15 \\
(8.3 \%)\end{array}$ & $(11.6 \%)$ & $\begin{array}{c}970 \\
(9.4 \%)\end{array}$ & $\begin{array}{c}79 \\
(10.7 \%)\end{array}$ & $\begin{array}{c}1,221 \\
(3.9 \%)\end{array}$ & $\begin{array}{c}55 \\
(4.9 \%)\end{array}$ & $\begin{array}{c}2,200) \\
(53 \%)\end{array}$ & (7.3\%) \\
\hline $50 \cdot 74 \%$ & $\begin{array}{c}15 \\
(8.2 \%)\end{array}$ & $(8.7 \%)$ & $\begin{array}{c}1,054 \\
(10.2 \%)\end{array}$ & $\begin{array}{c}68 \\
(9.2 \%)\end{array}$ & $\begin{array}{c}2,393 \\
(7.7 \%)\end{array}$ & $\begin{array}{c}110 \\
(9.9 \%)\end{array}$ & $\begin{array}{c}3,462 \\
(23 \%)\end{array}$ & (9.6\%) \\
\hline $75 \cdot 100 \%$ & $\begin{array}{c}13 \\
(6.8 \%)\end{array}$ & $(4.1 \%)$ & $\begin{array}{c}713 \\
(6.9 \%)\end{array}$ & $\begin{array}{c}48 \\
(6.5 \%)\end{array}$ & $\begin{array}{c}2,909 \\
(9.3 \%)\end{array}$ & $\begin{array}{c}100 \\
(9.0 \%)\end{array}$ & $\begin{array}{r}36035 \\
(87 \%)\end{array}$ & $\begin{array}{c}149 \\
(20 \%)\end{array}$ \\
\hline Unknown & $(3.3 \%)$ & $(4.4 \%)$ & $\begin{array}{c}865 \\
(84 \%)\end{array}$ & $\begin{array}{c}51 \\
(0.9 \%)\end{array}$ & $\begin{array}{c}7,032 \\
(225 \%)\end{array}$ & $\begin{array}{c}266 \\
(24.0 \%)\end{array}$ & $\begin{array}{c}7,904 \\
(189 \%)\end{array}$ & $\begin{array}{c}318 \\
(17.0 \%)\end{array}$ \\
\hline TOTALS & $(10000 \%)$ & $(1000 \%)$ & $\begin{array}{c}10,342 \\
(1000 \%)\end{array}$ & $\begin{array}{c}737 \\
(1000 \%)\end{array}$ & $\begin{array}{c}31,239 \\
(1000 \%)\end{array}$ & $\begin{array}{c}1,109 \\
(100.0 \%)\end{array}$ & $\begin{array}{c}41,768 \\
(1000 \%)\end{array}$ & $\begin{array}{r}1,872 \\
(100.0 \%)\end{array}$ \\
\hline \multicolumn{9}{|c|}{ Daily Percent Off Road } \\
\hline Under $10 \%$ & $\begin{array}{c}98 \\
(524 \%)\end{array}$ & $\begin{array}{c}15 \\
(58.0 \%)\end{array}$ & $\begin{array}{c}5,198 \\
(50.3 \%)\end{array}$ & $\begin{array}{c}392 \\
(53.2 \%)\end{array}$ & $\begin{array}{c}13,389 \\
(429 \%)\end{array}$ & $\begin{array}{c}467 \\
(42.1 \%)\end{array}$ & $\begin{array}{c}18.684 \\
(41.7 \%)\end{array}$ & $\begin{array}{r}874 \\
(467 \%)\end{array}$ \\
\hline $10 \cdot 24 \%$ & $\begin{array}{r}21 \\
(11.3 \%)\end{array}$ & $(11.2 \%)$ & $\begin{array}{c}1,372 \\
(13.3 \%)\end{array}$ & $\begin{array}{c}126 \\
(17.1 \%)\end{array}$ & $\begin{array}{c}2,791 \\
(8.9 \%)\end{array}$ & $\begin{array}{c}141 \\
(12.7 \%)\end{array}$ & $(10,185)$ &.$\quad(14 \%(\%)$ \\
\hline $25 \cdot 49 \%$ & $\begin{array}{c}9 \\
(4.6 \%)\end{array}$ & $(4.9 \%)$ & $\begin{array}{c}410 \\
(4.0 \%)\end{array}$ & $\begin{array}{c}40 \\
(5.4 \%)\end{array}$ & $\begin{array}{c}1,157 \\
(3.7 \%)\end{array}$ & $\begin{array}{c}65 \\
(5.9 \%)\end{array}$ & $\begin{array}{c}1,576 \\
(3.8 \%)\end{array}$ & $(5.7 \%)$ \\
\hline $50-74 \%$ & $\begin{array}{c}10 \\
(5.5 \%)\end{array}$ & $(4.0 \%)$ & $\begin{array}{c}503 \\
(4.9 \%)\end{array}$ & $\begin{array}{c}39 \\
(5.2 \%)\end{array}$ & $\begin{array}{l}1,298 \\
(4.2 \%)\end{array}$ & $\begin{array}{c}56 \\
(5.0 \%)\end{array}$ & $\begin{array}{c}18811 \\
(4.3 \%)\end{array}$ & $\begin{array}{c}96 \\
(5.1 \%)\end{array}$ \\
\hline $75-100 \%$ & $\begin{array}{c}37 \\
(19.5 \%)\end{array}$ & $(14.7 \%)$ & $\begin{array}{c}1,867 \\
(18.0 \%)\end{array}$ & $\begin{array}{c}75 \\
(10.2 \%)\end{array}$ & $\begin{array}{c}5,672 \\
(18.2 \%)\end{array}$ & $\begin{array}{c}135 \\
(12.2 \%)\end{array}$ & $\begin{array}{c}7,575 \\
(181 \%)\end{array}$ & $\begin{array}{r}214 \\
(11.4 \%)\end{array}$ \\
\hline Unknown & $\begin{array}{c}12 \\
(6.6 \%)\end{array}$ & $(7.1 \%)$ & $\begin{array}{c}992 \\
(9.6 \%)\end{array}$ & $\begin{array}{c}65 \\
(8.9 \%)\end{array}$ & $\begin{array}{c}6,932 \\
(22,2 \%)\end{array}$ & $\begin{array}{c}245 \\
(22.1 \%)\end{array}$ & $\begin{array}{r}7,936 \\
(19.0 \% 5)\end{array}$ & $\begin{array}{r}312 \\
(167 \%)\end{array}$ \\
\hline TOTALS & $\begin{array}{c}187 \\
(1000 \%)\end{array}$ & $(100.0 \%)$ & $\begin{array}{c}10,342 \\
(1000 \%)\end{array}$ & $\begin{array}{c}737 \\
(100.0 \%)\end{array}$ & $\begin{array}{c}31,239 \\
(1000 \%)\end{array}$ & $\begin{array}{c}1,109 \\
(100,0 \%)\end{array}$ & $\begin{array}{c}41,768 \\
(1000 \%)\end{array}$ & $\begin{array}{c}1,872 \\
(1000 \%)\end{array}$ \\
\hline
\end{tabular}

(a) Quantity rounded to zero. 
Table 3.20.

Distribution of Four Year and Older Trucks Over Daily Truck Description by Day of Week Operated: 1990 NTACS

\begin{tabular}{|c|c|c|c|c|c|c|c|}
\hline \multirow{2}{*}{$\begin{array}{l}\text { Daily } \\
\text { Truck } \\
\text { Description }\end{array}$} & \multicolumn{7}{|c|}{ Day of Week } \\
\hline & Sunday & Monday & Tuesday & Wednesday & Thursday & Friday & Saturday \\
\hline \multicolumn{8}{|l|}{ Straight Truck } \\
\hline $\begin{array}{l}\text { - With } 4 \text { Tires } \\
\text { Without Trailer }\end{array}$ & $90.1 \%$ & $82.0 \%$ & $81.9 \%$ & $82.5 \%$ & $82.3 \%$ & $82.5 \%$ & $87.0 \%$ \\
\hline $\begin{array}{l}\text { - With } 4 \text { Tires } \\
\text { With Trailer(s) }\end{array}$ & $1.5 \%$ & $1.5 \%$ & $1.9 \%$ & $1.7 \%$ & $1.7 \%$ & $1.7 \%$ & $1.7 \%$ \\
\hline $\begin{array}{l}\text { - With } 6 \text { or More Tires } \\
\text { Without Trailer }\end{array}$ & $1.3 \%$ & $6.1 \%$ & $6.3 \%$ & $6.2 \%$ & $6.2 \%$ & $6.0 \%$ & $2.9 \%$ \\
\hline $\begin{array}{l}\text { - With } 6 \text { or More Tires } \\
\text { With Trailer(s) }\end{array}$ & $0.2 \%$ & $0.7 \%$ & $0.7 \%$ & $0.8 \%$ & $0.6 \%$ & $0.6 \%$ & $0.3 \%$ \\
\hline \multicolumn{8}{|l|}{ Truck-Tractor } \\
\hline - With Trailer(s) & $0.8 \%$ & $2.2 \%$ & $2.3 \%$ & $2.3 \%$ & $2.3 \%$ & $2.2 \%$ & $0.9 \%$ \\
\hline - Without Trailer & (a) & (a) & (a) & (a) & (a) & (a) & (a) \\
\hline Other & $1.8 \%$ & $1.4 \%$ & $1.4 \%$ & $1.2 \%$ & $1.2 \%$ & $1.5 \%$ & $1.5 \%$ \\
\hline Unknown & $4.3 \%$ & $6.0 \%$ & $5.5 \%$ & $5.3 \%$ & $5.6 \%$ & $5.5 \%$ & $5.7 \%$ \\
\hline TOTALS & $100.0 \%$ & $1000 \%$ & $100.0 \%$ & $100.0 \%$ & $100.0 \%$ & $100.0 \%$ & $100.0 \%$ \\
\hline
\end{tabular}

(a) Quantity rounded to zero. 


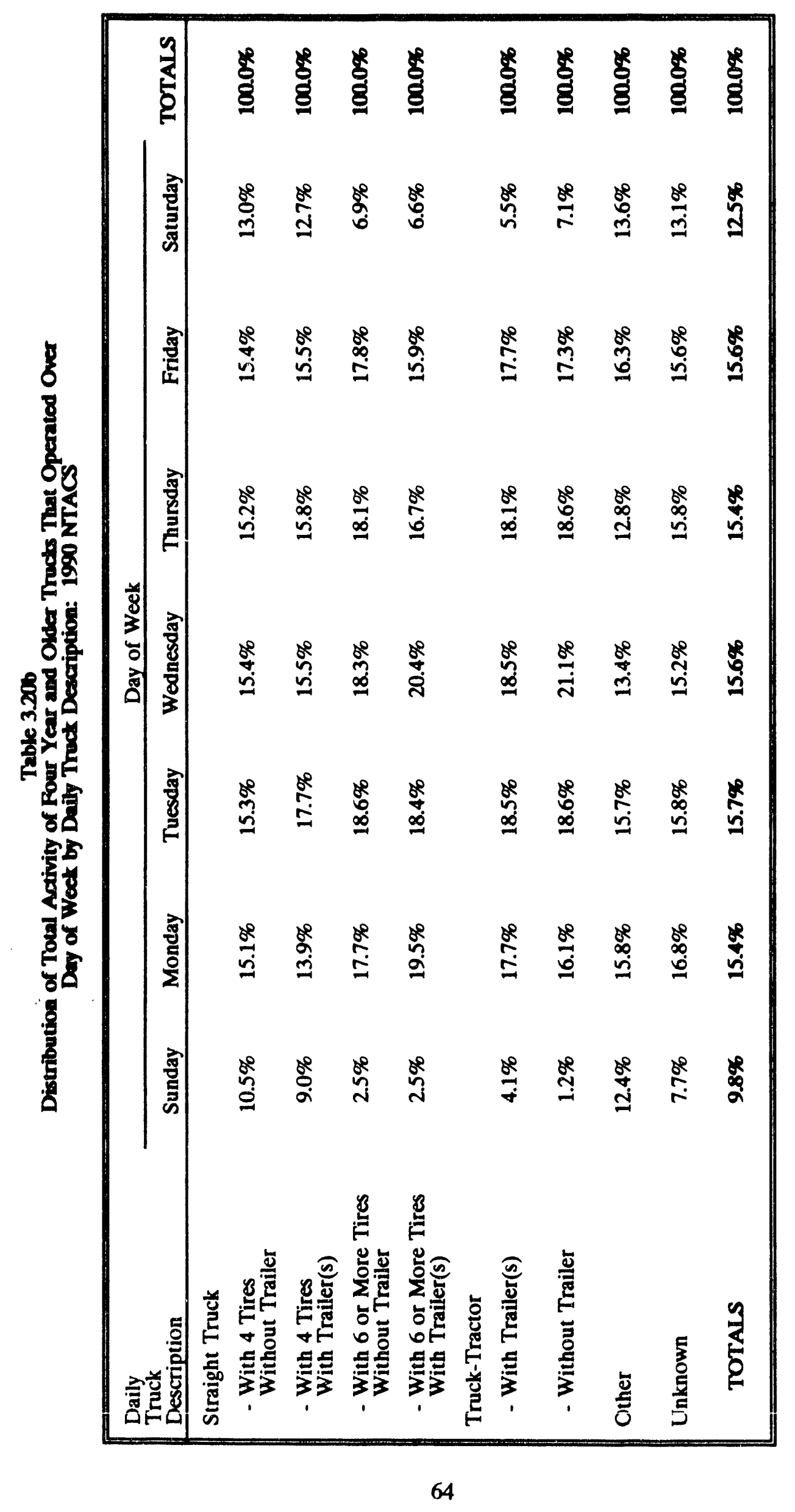




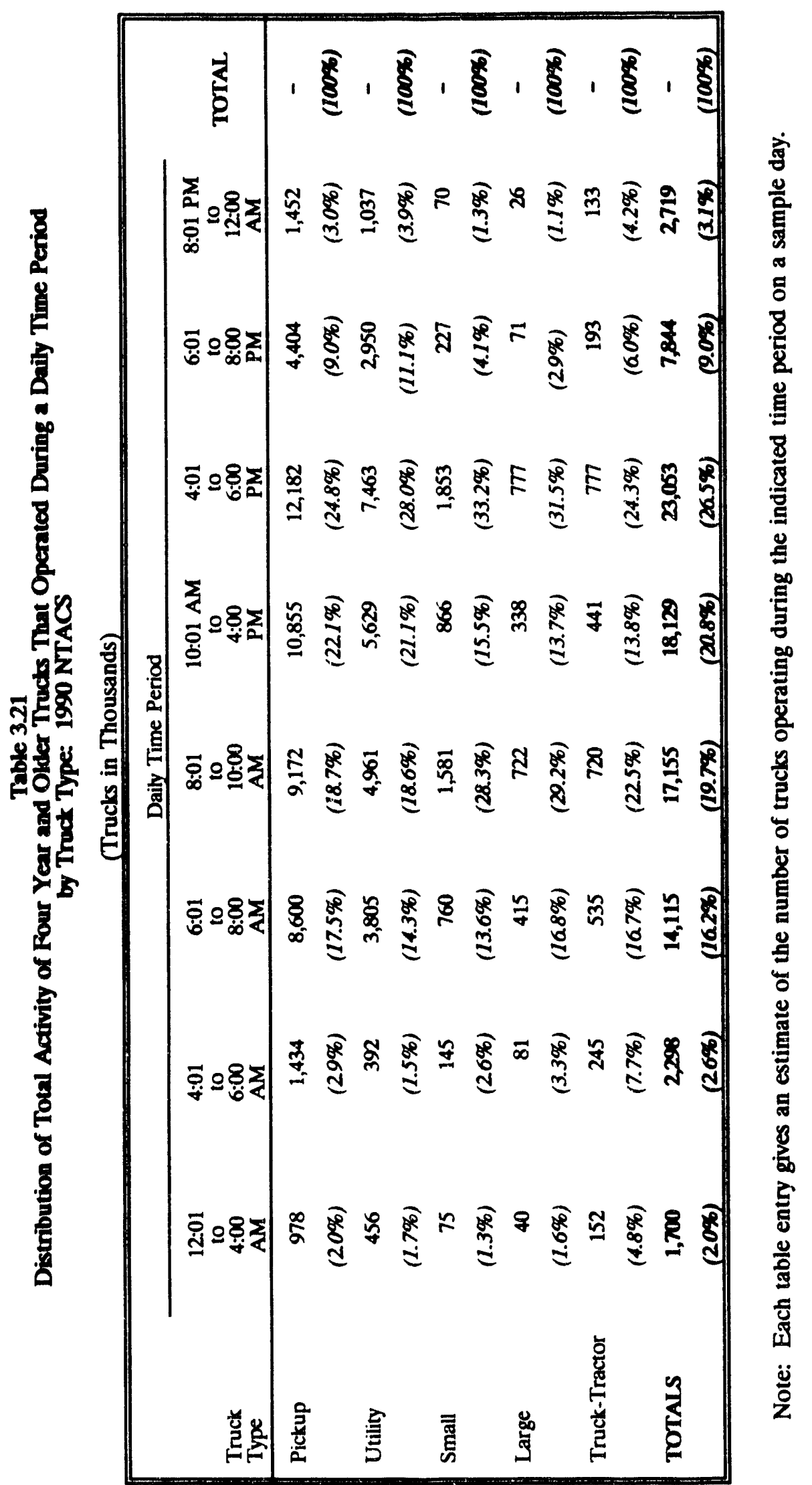




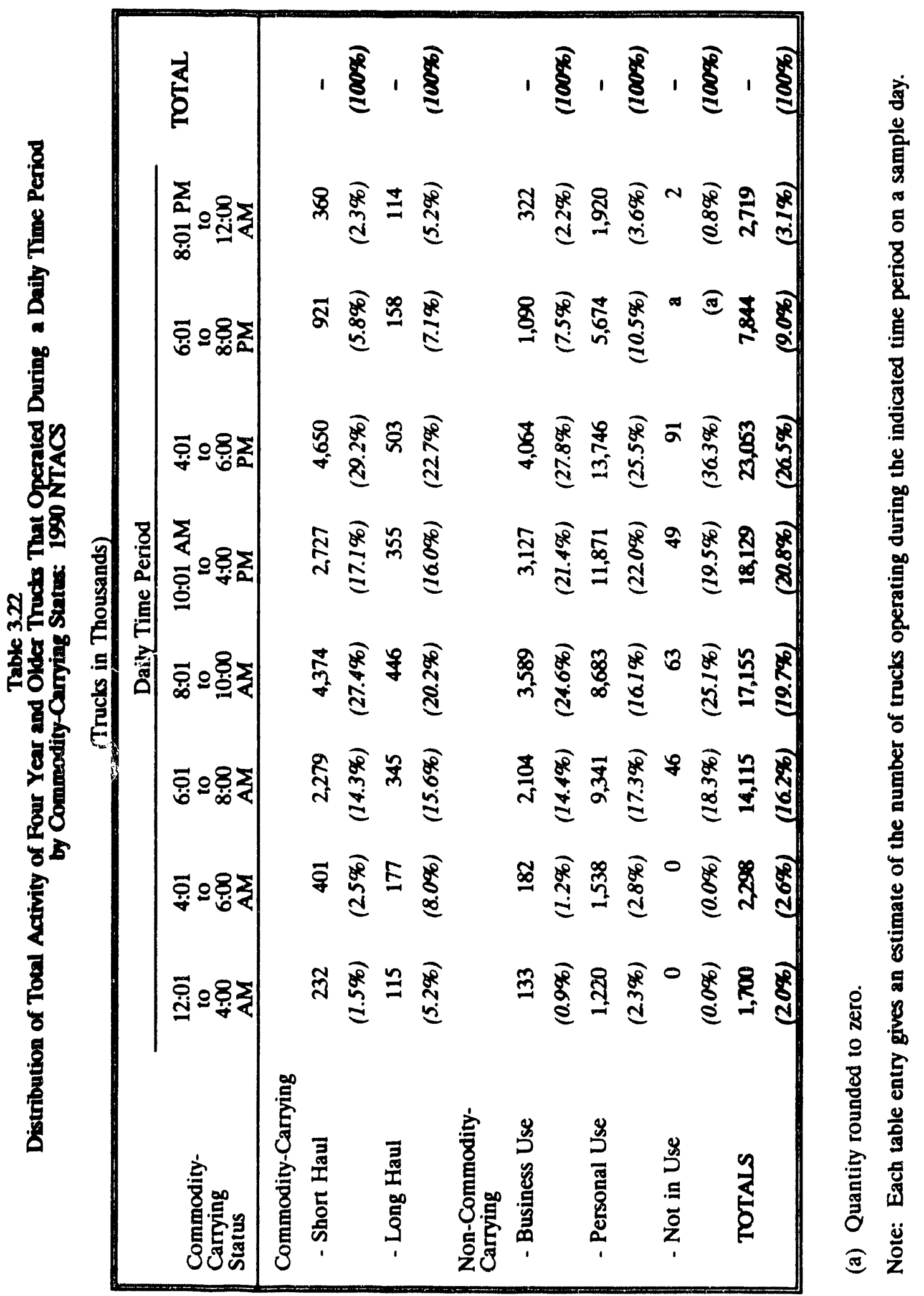




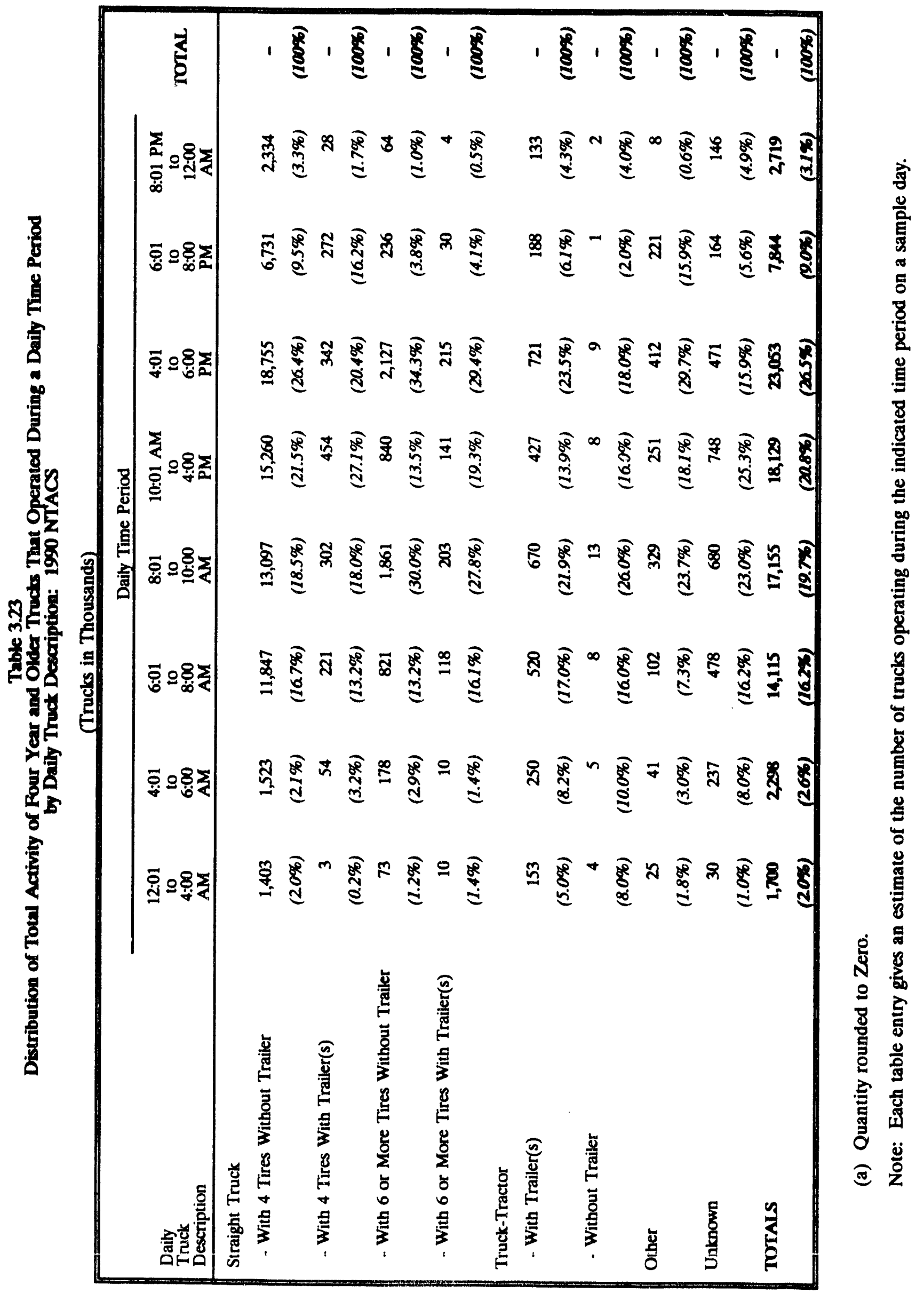


Table 3.24

Distribution of Four Year and Older Trucks

by Daily Percent Interstate, Daily Percent Four Lane, Daily Percent Off Road, and Commodity-Carrying Statua: 1990 NTACS

\begin{tabular}{|c|c|c|c|c|c|c|}
\hline & \multicolumn{5}{|c|}{ Commodity-Carrying Status } & \multirow{3}{*}{ TOTALS } \\
\hline & \multicolumn{2}{|c|}{ Commodity-Carrying } & \multicolumn{3}{|c|}{ Non-Commodity-Carrying } & \\
\hline & $\begin{array}{l}\text { Short } \\
\text { Haul }\end{array}$ & $\begin{array}{l}\text { Long } \\
\text { Haul }\end{array}$ & $\begin{array}{c}\text { Business } \\
\text { Use }\end{array}$ & $\begin{array}{c}\text { Personal } \\
\text { Use }\end{array}$ & $\begin{array}{l}\text { Not in } \\
\text { Use }\end{array}$ & \\
\hline \multicolumn{7}{|c|}{ Daily Percent Interstate } \\
\hline Under $10 \%$ & $58.0 \%$ & $30.2 \%$ & $59.2 \%$ & $50.4 \%$ & $72.1 \%$ & $528 \%$ \\
\hline $10-24 \%$ & $6.5 \%$ & $3.3 \%$ & $6.2 \%$ & $5.7 \%$ & $0.0 \%$ & $5.9 \%$ \\
\hline $25-49 \%$ & $4.7 \%$ & $5.8 \%$ & $3.6 \%$ & $5.3 \%$ & $0.0 \%$ & $4.9 \%$ \\
\hline $50-74 \%$ & $7.2 \%$ & $11.6 \%$ & $7.4 \%$ & $6.6 \%$ & $1.2 \%$ & $6.9 \%$ \\
\hline $75 \cdot 100 \%$ & $9.0 \%$ & $37.9 \%$ & $8.5 \%$ & $11.4 \%$ & $4.9 \%$ & $11.0 \%$ \\
\hline Unknown & $14.5 \%$ & $11.1 \%$ & $15.1 \%$ & $20.6 \%$ & $21.8 \%$ & $185 \%$ \\
\hline TOTAIS & $100.0 \%$ & $100.0 \%$ & $100.0 \%$ & $100.0 \%$ & $100.0 \%$ & $100.0 \%$ \\
\hline \multicolumn{7}{|c|}{ Daily Percent Four Lane } \\
\hline Under $10 \%$ & $52.9 \%$ & $48.6 \%$ & $49.9 \%$ & $47.9 \%$ & $35.8 \%$ & $49.0 \%$ \\
\hline $10 \cdot 24 \%$ & $9.6 \%$ & $16.8 \%$ & $10.1 \%$ & $9.5 \%$ & $21.8 \%$ & $98 \%$ \\
\hline $25-49 \%$ & $6.3 \%$ & $8.1 \%$ & $7.8 \%$ & $4.3 \%$ & $14.1 \%$ & $5.3 \%$ \\
\hline $50-74 \%$ & $8.0 \%$ & $6.7 \%$ & $9.4 \%$ & $8.2 \%$ & $9.6 \%$ & $83 \%$ \\
\hline $75-100 \%$ & $8.0 \%$ & $6.5 \%$ & $7.0 \%$ & $9.4 \%$ & $5.3 \%$ & $87 \%$ \\
\hline Unknown & $15.3 \%$ & $13.2 \%$ & $15.8 \%$ & $20.8 \%$ & $13.4 \%$ & $18.9 \%$ \\
\hline TOTALS & $100.0 \%$ & $1000 \%$ & $1000 \%$ & $1000 \%$ & $100.0 \%$ & $100.0 \%$ \\
\hline \multicolumn{7}{|c|}{ Daily Percent Off Road } \\
\hline Under $10 \%$ & $41.1 \%$ & $58.8 \%$ & $43.2 \%$ & $45.8 \%$ & $32.3 \%$ & $44.7 \%$ \\
\hline $10-24 \%$ & $10.1 \%$ & $9.7 \%$ & $11.5 \%$ & $9.6 \%$ & $14.9 \%$ & $10.0 \%$ \\
\hline $25 \cdot 49 \%$ & $4.6 \%$ & $4.1 \%$ & $5.9 \%$ & $3.0 \%$ & $9.6 \%$ & $38 \%$ \\
\hline $50 \cdot 74 \%$ & $5.6 \%$ & $4.1 \%$ & $4.0 \%$ & $4.1 \%$ & $0.0 \%$ & $43 \%$ \\
\hline $75-100 \%$ & $22.1 \%$ & $7.9 \%$ & $19.5 \%$ & $17.0 \%$ & $30.6 \%$ & $18.1 \%$ \\
\hline Unknown & $16.5 \%$ & $15.4 \%$ & $15.9 \%$ & $20.5 \%$ & $12.5 \%$ & $19.0 \%$ \\
\hline TOTALS & $100.0 \%$ & $100.0 \%$ & $100.0 \%$ & $100.0 \%$ & $100.0 \%$ & $100.0 \%$ \\
\hline
\end{tabular}


Table 3.25

Distribution of Four Year and Older Trucks

by Daily Percent Interstate, Daily Percent Four Lane, Daily Percent Off Road, and Daily Major Use: 1990 NTACS

\begin{tabular}{|c|c|c|c|c|c|c|}
\hline & \multicolumn{5}{|c|}{ Daily Major Use } & \multirow[b]{2}{*}{ TOTALS } \\
\hline & $\begin{array}{c}\text { Personal } \\
\text { Transportation } \\
\end{array}$ & $\begin{array}{c}\text { Contract } \\
\text { Carrier } \\
\end{array}$ & $\begin{array}{c}\text { Common } \\
\text { Carrier } \\
\end{array}$ & $\begin{array}{c}\text { Other } \\
\text { Business Use } \\
\end{array}$ & Unknown & \\
\hline \multicolumn{7}{|c|}{ Daily Percent Interstate } \\
\hline Under $10 \%$ & $55.3 \%$ & $31.8 \%$ & $42.2 \%$ & $58.8 \%$ & $22.9 \%$ & $528 \%$ \\
\hline $10-24 \%$ & $5.7 \%$ & $11.9 \%$ & $6.2 \%$ & $8.1 \%$ & $0.8 \%$ & $5.9 \%$ \\
\hline $25-49 \%$ & $5.2 \%$ & $5.8 \%$ & $6.4 \%$ & $5.1 \%$ & $0.5 \%$ & $4.9 \%$ \\
\hline $50-74 \%$ & $6.6 \%$ & $9.1 \%$ & $12.3 \%$ & $9.2 \%$ & $1.7 \%$ & 6.99 \\
\hline $75-100 \%$ & $11.5 \%$ & $21.5 \%$ & $27.6 \%$ & $9.1 \%$ & $1.2 \%$ & $11.0 \%$ \\
\hline Unknown & $15.6 \%$ & $20.0 \%$ & $5.3 \%$ & $9.6 \%$ & $72.9 \%$ & $185 \%$ \\
\hline TOTALS & $100.0 \%$ & $100.0 \%$ & $1000 \%$ & $1000 \%$ & $100.0 \%$ & $1000 \%$ \\
\hline \multicolumn{7}{|c|}{ Daily Percent Four Iane } \\
\hline Under $10 \%$ & $52.6 \%$ & $41.4 \%$ & $48.4 \%$ & $49.4 \%$ & $15.7 \%$ & $49.0 \%$ \\
\hline $10 \cdot 24 \%$ & $9.2 \%$ & $10.5 \%$ & $21.4 \%$ & $13.5 \%$ & $1.7 \%$ & $98 \%$ \\
\hline $25-49 \%$ & $4.3 \%$ & $11.6 \%$ & $8.0 \%$ & $10.2 \%$ & $1.3 \%$ & $53 \%$ \\
\hline $50-74 \%$ & $8.6 \%$ & $10.9 \%$ & $7.7 \%$ & $9.6 \%$ & $2.1 \%$ & $83 \%$ \\
\hline $75-100 \%$ & $9.7 \%$ & $4.4 \%$ & $7.1 \%$ & $6.8 \%$ & $5.0 \%$ & 8.7\% \\
\hline Unknown & $15.7 \%$ & $21.2 \%$ & $7.2 \%$ & $10.5 \%$ & $74.2 \%$ & $189 \%$ \\
\hline TOTAIS & $1000 x$ & $1000 \times$ & 1000x & $100.0 \%$ & $100.0 x$ & $1000 x$ \\
\hline \multicolumn{7}{|c|}{ Daily Percent Off Road } \\
\hline Under $10 \%$ & $47.4 \%$ & $45.4 \%$ & $43.8 \%$ & $47.2 \%$ & $13.6 \%$ & $4.7 \%$ \\
\hline $10-24 \%$ & $10.0 \%$ & $14.1 \%$ & $20.4 \%$ & $11.9 \%$ & $0.7 \%$ & $100 \%$ \\
\hline $25-49 \%$ & $3.7 \%$ & $5.4 \%$ & $5.3 \%$ & $4.3 \%$ & $1.7 \%$ & $38 \%$ \\
\hline $50-74 \%$ & $4.2 \%$ & $3.9 \%$ & $8.3 \%$ & $5.1 \%$ & $2.6 \%$ & $43 \%$ \\
\hline $75-100 \%$ & $19.3 \%$ & $8.6 \%$ & $12.6 \%$ & $20.6 \%$ & $5.7 \%$ & $18.1 \%$ \\
\hline Unknown & $15.5 \%$ & $225 \%$ & $9.6 \%$ & $10.8 \%$ & $75.7 \%$ & $19.0 \%$ \\
\hline TOTAIS & $100.0 \%$ & $100.0 \%$ & $100.0 \%$ & $100.0 \%$ & $100.0 \%$ & $100.0 \%$ \\
\hline
\end{tabular}


Table 3.26

Distribution of Pour Year and Older Trucks

by Daily Percent Interstate, Daily Percent Pour Lane, Daily Percent Off Road, and Daily Truck Description: 1990 NTACS

\begin{tabular}{|c|c|c|c|c|c|c|c|c|c|}
\hline & \multicolumn{4}{|c|}{ Straight Truck } & & & \multirow[b]{3}{*}{ Other } & \multirow[b]{3}{*}{ Unknown } & \multirow[b]{3}{*}{ TOTANs } \\
\hline & \multicolumn{2}{|c|}{4 Tires } & \multicolumn{2}{|c|}{6 or More Tires } & \multicolumn{2}{|c|}{ Truck-Tractor } & & & \\
\hline & $\begin{array}{c}\text { Without } \\
\text { Trailer }\end{array}$ & $\begin{array}{r}\text { With } \\
\text { Trailer(a) } \\
\end{array}$ & $\begin{array}{c}\text { Without } \\
\text { Trailer } \\
\end{array}$ & $\begin{array}{r}\text { With } \\
\text { Trailer(s) } \\
\end{array}$ & $\begin{array}{r}\text { With } \\
\text { Trailer(s) } \\
\end{array}$ & $\begin{array}{c}\text { Without } \\
\text { Trailer }\end{array}$ & & & \\
\hline \multicolumn{10}{|c|}{ Daily Percent Interstate } \\
\hline $10 \cdot 24 \%$ & $6.0 \%$ & $13.0 \%$ & $6.7 \%$ & $5.3 \%$ & $6.7 \%$ & $12.4 \%$ & $11.1 \%$ & $0.5 \%$ & $5.9 \%$ \\
\hline $25 \cdot 49 \%$ & $4.9 \%$ & $12.5 \%$ & $5.0 \%$ & $5.1 \%$ & $8.1 \%$ & $1.5 \%$ & $14.3 \%$ & $0.3 \%$ & $4.9 \%$ \\
\hline $50 \cdot 74 \%$ & $7.1 \%$ & $10.8 \%$ & $7.6 \%$ & $7.4 \%$ & $15.5 \%$ & $12.9 \%$ & $3.1 \%$ & $1.6 \%$ & c9s \\
\hline $75 \cdot 100 \%$ & $11.8 \%$ & $1.2 \%$ & $7.4 \%$ & $26.5 \%$ & $34.6 \%$ & $10.9 \%$ & $1.9 \%$ & $1.0 \%$ & $11.0 \%$ \\
\hline Under $10 \%$ & $51.7 \%$ & $46.7 \%$ & $54.7 \%$ & $60.6 \%$ & $49.0 \%$ & $44.6 \%$ & $44.4 \%$ & $15.3 \%$ & $490 x$ \\
\hline $10-24 \%$ & $9.9 \%$ & $13.0 \%$ & $12.4 \%$ & $12.1 \%$ & $17.5 \%$ & $21.4 \%$ & $13.1 \%$ & $1.6 \%$ & $98 \%$ \\
\hline $25 \cdot 49 \%$ & $5.5 \%$ & $8.1 \%$ & $5.4 \%$ & $6.8 \%$ & $10.8 \%$ & $7.0 \%$ & $3.9 \%$ & $0.9 \%$ & $5.3 \%$ \\
\hline $50 \cdot 74 \%$ & $8.3 \%$ & $13.0 \%$ & $10.0 \%$ & $8.1 \%$ & $7.8 \%$ & $11.5 \%$ & $25.8 \%$ & $1.9 \%$ & $8.3 \%$ \\
\hline $75 \cdot 100 \%$ & $9.6 \%$ & $0.8 \%$ & $6.7 \%$ & $4.5 \%$ & 7.49 & $1.9 \%$ & $0.9 \%$ & $5.3 \%$ & 8.7\% \\
\hline Unknown & $14.9 \%$ & $18.4 \%$ & $10.8 \%$ & $7.9 \%$ & $7.5 \%$ & $13.6 \%$ & $11.8 \%$ & $75.1 \%$ & $18.9 \%$ \\
\hline TOTALS & $100.0 \%$ & $100.0 \% 5$ & 100ex & $100.0 x$ & $100.0 \%$ & $100.0 \%$ & $100.0 x$ & $1000 x$ & $100.0 x$ \\
\hline Unknown & $14.5 \%$ & $18.3 \%$ & $12.4 \%$ & $19.3 \%$ & $9.6 \%$ & $8.2 \%$ & $13.9 \%$ & $76.9 \%$ & $19.0 \%$ \\
\hline TOTANS & $1000 x$ & $1000 \%$ & $1000 x$ & $1000 \%$ & $100.0 \%$ & $1000 x$ & $1000 x$ & $1000 x$ & $100.0 x$ \\
\hline
\end{tabular}




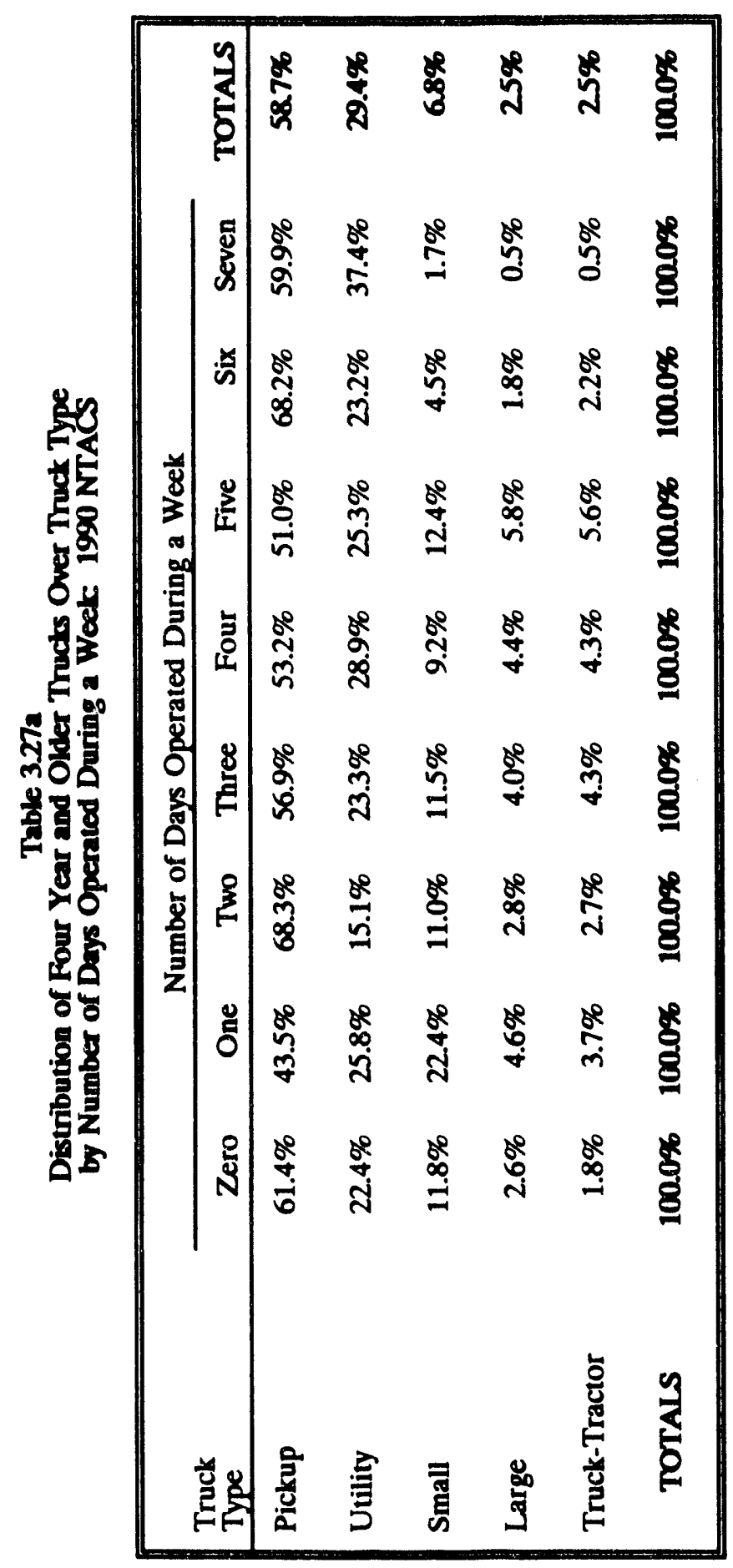




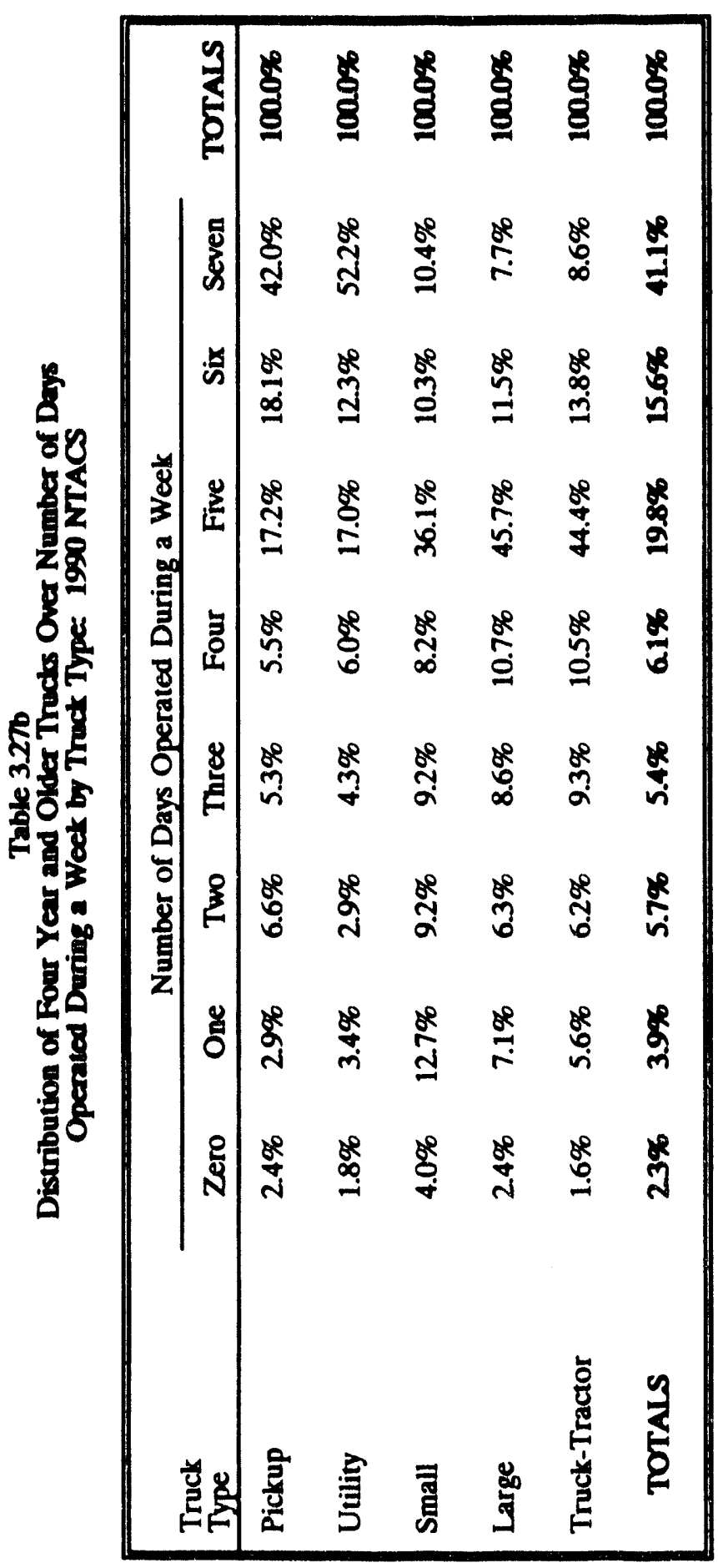


Table 3.28a

Distribution of Four Year and Older Tructs Over Truck Model Year by Number of Days Operated During a Week: 1990 NTACS

\begin{tabular}{|c|c|c|c|c|c|c|c|c|c|}
\hline \multirow{2}{*}{$\begin{array}{l}\text { Truck } \\
\text { Model } \\
\text { Year }\end{array}$} & \multicolumn{8}{|c|}{ Number of Days Operated During a Week } & \multirow[b]{2}{*}{ TOTALS } \\
\hline & Zero & One & Two & Three & Four & Five & Six & Seven & \\
\hline 1987(a) & $0.1 \%$ & $3.9 \%$ & $1.7 \%$ & $2.5 \%$ & $3.7 \%$ & $10.2 \%$ & $9.7 \%$ & $12.1 \%$ & 9.1\% \\
\hline 1986 & $3.6 \%$ & $8.7 \%$ & $6.8 \%$ & $9.9 \%$ & $8.1 \%$ & $16.5 \%$ & $10.5 \%$ & $16.2 \%$ & $13.4 \%$ \\
\hline 1985 & $5.4 \%$ & $6.7 \%$ & $10.3 \%$ & $18.5 \%$ & $8.0 \%$ & $9.7 \%$ & $7.6 \%$ & $10.6 \%$ & $10.0 \%$ \\
\hline 1984 & $0.7 \%$ & $5.9 \%$ & $2.0 \%$ & $4.7 \%$ & $8.8 \%$ & $9.0 \%$ & $10.1 \%$ & $10.4 \%$ & $88 \%$ \\
\hline 1983 & $2.7 \%$ & $0.4 \%$ & $5.4 \%$ & $5.4 \%$ & $5.6 \%$ & $6.4 \%$ & $8.6 \%$ & $9.8 \%$ & $7.7 \%$ \\
\hline 1982 & $1.6 \%$ & $5.3 \%$ & $2.4 \%$ & $6.6 \%$ & $12.8 \%$ & $8.5 \%$ & $3.5 \%$ & $4.9 \%$ & $5.8 \%$ \\
\hline 1981 & $13.5 \%$ & $5.7 \%$ & $3.4 \%$ & $2.3 \%$ & $2.5 \%$ & $2.7 \%$ & $7.1 \%$ & $4.1 \%$ & $4.3 \%$ \\
\hline 1980 & $0.3 \%$ & $2.6 \%$ & $0.7 \%$ & $5.2 \%$ & $4.7 \%$ & $3.9 \%$ & $2.4 \%$ & $2.5 \%$ & $29 \%$ \\
\hline 1979 & $5.4 \%$ & $14.1 \%$ & $22.0 \%$ & $4.6 \%$ & $5.9 \%$ & $5.2 \%$ & $10.1 \%$ & $6.2 \%$ & $7.7 \%$ \\
\hline 1978 & $3.4 \%$ & $9.1 \%$ & $17.5 \%$ & $11.0 \%$ & $8.1 \%$ & $5.0 \%$ & $3.0 \%$ & $4.7 \%$ & $5.9 \%$ \\
\hline Pre-1978 & $63.3 \%$ & $37.6 \%$ & $27.8 \%$ & $29.2 \%$ & $31.9 \%$ & $22.9 \%$ & $27.4 \%$ & $18.4 \%$ & $24.4 \%$ \\
\hline Unknown & $0.0 \%$ & (b) & (b) & (b) & $0.0 \%$ & (b) & $0.0 \%$ & $0.0 \%$ & (b) \\
\hline TOTALS & $100.0 \%$ & $100.0 \%$ & $100.0 \%$ & $100.0 \%$ & $100.0 \%$ & $100.0 \%$ & $100.0 \%$ & $100.0 \%$ & $100.0 \%$ \\
\hline
\end{tabular}

(a) Includes some 1988 models.

(b) Quantity rounded to zero. 
Table 3.250

Distribution of Four Year and Older Tructs Over Number of Days Operated During a Week by Truct Model Year: 1990 NTACS

\begin{tabular}{|c|c|c|c|c|c|c|c|c|c|}
\hline \multirow{2}{*}{$\begin{array}{l}\text { Truck } \\
\text { Model } \\
\text { Year } \\
\end{array}$} & \multicolumn{8}{|c|}{ Number of Days Operated During a Week } & \multirow[b]{2}{*}{ TOTALS } \\
\hline & Zero & One & Two & Three & Four & Five & Six & Seven & \\
\hline 1987(a) & (b) & $1.6 \%$ & $1.1 \%$ & $1.5 \%$ & $2.5 \%$ & $22.1 \%$ & $16.1 \%$ & $54.5 \%$ & $100.0 \%$ \\
\hline 1986 & $0.6 \%$ & $2.5 \%$ & $2.9 \%$ & $4.0 \%$ & $3.7 \%$ & $24.4 \%$ & $12.2 \%$ & $49.7 \%$ & $100.0 \%$ \\
\hline 1985 & $1.2 \%$ & $2.6 \%$ & $5.9 \%$ & $10.1 \%$ & $4.9 \%$ & $19.4 \%$ & $12.0 \%$ & $43.9 \%$ & $100.0 \%$ \\
\hline 1984 & $0.2 \%$ & $2.6 \%$ & $1.3 \%$ & $2.9 \%$ & $6.1 \%$ & $20.4 \%$ & $17.9 \%$ & $48.7 \%$ & $100.0 \%$ \\
\hline 1983 & $0.8 \%$ & $0.2 \%$ & $4.0 \%$ & $3.9 \%$ & $4.4 \%$ & $16.6 \%$ & $17.4 \%$ & $52.7 \%$ & $100.0 \%$ \\
\hline 1982 & $0.6 \%$ & $3.5 \%$ & $2.4 \%$ & $6.2 \%$ & $13.5 \%$ & $29.1 \%$ & $9.6 \%$ & $35.1 \%$ & $1000 \%$ \\
\hline 1981 & $7.2 \%$ & $5.1 \%$ & $4.4 \%$ & $2.9 \%$ & $3.5 \%$ & $12.4 \%$ & $25.5 \%$ & $39.0 \%$ & $1000 \%$ \\
\hline 1980 & $0.2 \%$ & $3.4 \%$ & $1.4 \%$ & $9.8 \%$ & $9.9 \%$ & $26.4 \%$ & $13.0 \%$ & $36.0 \%$ & $100.0 \%$ \\
\hline 1979 & $1.6 \%$ & $7.1 \%$ & $16.3 \%$ & $3.3 \%$ & $4.7 \%$ & $13.3 \%$ & $20.6 \%$ & $33.1 \%$ & $100.0 \%$ \\
\hline 1978 & $1.3 \%$ & $5.9 \%$ & $16.8 \%$ & $10.1 \%$ & $8.4 \%$ & $16.8 \%$ & $8.0 \%$ & $32.6 \%$ & $1000 \%$ \\
\hline Pre-1978 & $6.0 \%$ & $5.9 \%$ & $6.5 \%$ & $6.5 \%$ & $8.0 \%$ & $18.6 \%$ & $17.5 \%$ & $31.0 \%$ & $100.0 \%$ \\
\hline Unknown & $0.0 \%$ & (b) & (b) & (b) & $0.0 \%$ & $100.0 \%$ & $0.0 \%$ & $0.0 \%$ & $100.0 \%$ \\
\hline TOTALS & $23 \%$ & $3.9 \%$ & $5.7 \%$ & $5.4 \%$ & $6.1 \%$ & $90.8 \%$ & $15.6 \%$ & $41.1 \%$ & $100.0 \%$ \\
\hline
\end{tabular}

(a) Includes some 1988 models.

(b) Quantity rounded to zero. 
Table 3.29

Number of Four Year and Older Trucks

by Annual Major Use and Daily Major Use: 1990 NTACS

(Trucks in Thousands)

\begin{tabular}{|c|c|c|c|c|c|c|}
\hline \multirow[b]{2}{*}{$\begin{array}{l}\text { Annual } \\
\text { Major } \\
\text { Use } \\
\end{array}$} & \multicolumn{5}{|c|}{ Daily Major Use } & \multirow[b]{2}{*}{$\frac{\text { TOTALS }}{\text { Truecs }}$} \\
\hline & $\begin{array}{c}\text { Personal } \\
\text { Transportation } \\
\text { Trucks }\end{array}$ & $\begin{array}{l}\text { Contract } \\
\text { Carrier } \\
\text { Trucks }\end{array}$ & $\begin{array}{l}\text { Common } \\
\text { Carrier } \\
\text { Trucks }\end{array}$ & $\begin{array}{c}\text { Other } \\
\text { Business Use } \\
\text { Trucks }\end{array}$ & $\frac{\text { Unknown }}{\text { Trucks }}$ & \\
\hline $\begin{array}{l}\text { Personal } \\
\text { Transportation }\end{array}$ & $\begin{array}{r}29,389 \\
(98.8 \%)\end{array}$ & $\begin{array}{r}0 \\
(0.0 \%)\end{array}$ & $\begin{array}{r}0 \\
(0.0 \%)\end{array}$ & $\begin{array}{r}70 \\
(1.0 \%)\end{array}$ & $\begin{array}{r}2,146 \\
(70.3 \%)\end{array}$ & $\begin{array}{r}31,605 \\
(75.790)\end{array}$ \\
\hline $\begin{array}{l}\text { Contract } \\
\text { Carrier }\end{array}$ & $\begin{array}{r}0 \\
(0.0 \%)\end{array}$ & $\begin{array}{r}634 \\
(98.2 \%)\end{array}$ & $\begin{array}{r}2 \\
(0.1 \%)\end{array}$ & $\begin{array}{r}2 \\
\text { (a) }\end{array}$ & $\begin{array}{r}19 \\
(0.6 \%)\end{array}$ & $\begin{array}{r}656 \\
(1.6 \%)\end{array}$ \\
\hline $\begin{array}{l}\text { Common } \\
\text { Carrier }\end{array}$ & $\begin{array}{r}16 \\
(0.1 \%)\end{array}$ & $\begin{array}{r}6 \\
(0.9 \%)\end{array}$ & $\begin{array}{r}1,305 \\
(99.3 \%)\end{array}$ & $\begin{array}{r}14 \\
(0.2 \%)\end{array}$ & $\begin{array}{r}77 \\
(2.5 \%)\end{array}$ & $\begin{array}{r}1,419 \\
(3.4 \%)\end{array}$ \\
\hline $\begin{array}{l}\text { Other } \\
\text { Business Use }\end{array}$ & $\begin{array}{r}112 \\
(0.4 \%)\end{array}$ & $\begin{array}{r}3 \\
(0.5 \%)\end{array}$ & $\begin{array}{r}2 \\
(0.2 \%)\end{array}$ & $\begin{array}{r}6,859 \\
(98.0 \% 6)\end{array}$ & $\begin{array}{r}371 \\
(12.2 \%)\end{array}$ & $\begin{array}{r}7,347 \\
(17.6 \%)\end{array}$ \\
\hline Unknown & $\begin{array}{r}244 \\
(0.8 \%)\end{array}$ & $\begin{array}{r}3 \\
(0.4 \%)\end{array}$ & $\begin{array}{r}6 \\
(0.4 \%)\end{array}$ & $\begin{array}{r}51 \\
(0.7 \%)\end{array}$ & $\begin{array}{r}438 \\
(14.3 \%)\end{array}$ & $\begin{array}{r}741 \\
(1.8 \%)\end{array}$ \\
\hline TOTALS & $\begin{array}{r}29,760 \\
(100.0 \%)\end{array}$ & $\begin{array}{r}645 \\
(100.0 \%)\end{array}$ & $\begin{array}{r}1,314 \\
(100,0 \%)\end{array}$ & $\begin{array}{r}6,996 \\
(100.0 \%)\end{array}$ & $\begin{array}{r}3,051 \\
(100.0 \%)\end{array}$ & $\begin{array}{r}41,768 \\
(100.0 \%)\end{array}$ \\
\hline
\end{tabular}

(a) Quantity rounded to zero. 
Table 3.30

Number of Four Year and Older Tructs

by Annual Truct Description and Daily Truct Description: 1990 NTACS

(Trucks in Thousands)

\begin{tabular}{|c|c|c|c|c|c|c|c|c|c|}
\hline \multirow[b]{4}{*}{$\begin{array}{l}\text { Annual } \\
\text { Truck } \\
\text { Description }\end{array}$} & \multicolumn{9}{|c|}{ Daily Truck Description } \\
\hline & \multicolumn{4}{|c|}{ Straight Truck } & \multirow{2}{*}{\multicolumn{2}{|c|}{ Truck-Tractor }} & \multirow[b]{3}{*}{$\begin{array}{l}\text { Other } \\
\text { Truckss }\end{array}$} & \multirow[b]{3}{*}{$\frac{\text { Unknown }}{\text { Trucks }}$} & \multirow[b]{3}{*}{$\frac{\text { TOTALS }}{\text { Tructs }}$} \\
\hline & \multicolumn{2}{|c|}{4 Tires } & \multicolumn{2}{|c|}{6 or More Tires } & & & & & \\
\hline & $\begin{array}{l}\text { Without } \\
\text { Trailer } \\
\text { Trucks } \\
\end{array}$ & $\begin{array}{c}\text { With } \\
\text { Trailer(s) } \\
\text { Truckes } \\
\end{array}$ & $\begin{array}{l}\text { Without } \\
\text { Trailer } \\
\text { Trucks } \\
\end{array}$ & $\begin{array}{c}\text { With } \\
\text { Traiter(s) } \\
\text { Trucks } \\
\end{array}$ & $\begin{array}{c}\text { With } \\
\text { Trailer(s) } \\
\text { Trucks }\end{array}$ & $\begin{array}{l}\text { Without } \\
\text { Trailer } \\
\text { Trucks }\end{array}$ & & & \\
\hline \multicolumn{10}{|l|}{ Straight Truck } \\
\hline $\begin{array}{l}\text { - With } 4 \text { Tires } \\
\text { Without Trailer }\end{array}$ & $\begin{array}{r}32,341 \\
(97.5 \%)\end{array}$ & $\begin{array}{r}80 \\
(11.5 \%)\end{array}$ & (a) & $\begin{array}{r}0 \\
(0.0 \%)\end{array}$ & $\begin{array}{r}0 \\
(0.0 \%)\end{array}$ & $(0.0 \%)$ & $(0.7 \%$ & $\begin{array}{r}2,671 \\
(85.4 \%)\end{array}$ & $\begin{array}{r}35,097 \\
(84.0 \%)\end{array}$ \\
\hline $\begin{array}{l}\text { - With } 4 \text { Tires } \\
\text { With Trailer(s) }\end{array}$ & $\begin{array}{r}575 \\
(1.7 \%)\end{array}$ & $\begin{array}{r}519 \\
(74.8 \%)\end{array}$ & $\begin{array}{r}0 \\
(0.0 \%)\end{array}$ & $\begin{array}{r}0 \\
(0.0 \%)\end{array}$ & $\begin{array}{r}0 \\
(0.0 \%)\end{array}$ & $\begin{array}{r}0 \\
(0.0 \%)\end{array}$ & $\begin{array}{r}0 \\
(0.0 \%)\end{array}$ & $\begin{array}{r}69 \\
(22 \%)\end{array}$ & $\begin{array}{r}1,163 \\
(28 \%)\end{array}$ \\
\hline $\begin{array}{l}\text { With } 6 \text { or More Tires } \\
\text { Without Trailer }\end{array}$ & (a) & $\begin{array}{r}0 \\
(0.0 \%)\end{array}$ & $\begin{array}{r}2,810 \\
(98.2 \%)\end{array}$ & $(28 \%)^{9}$ & $\begin{array}{r}0 \\
(0.0 \%)\end{array}$ & $(0.0 \%)$ & $\begin{array}{r}18 \\
(3.0 \%)\end{array}$ & $\begin{array}{r}210 \\
(6.7 \%)\end{array}$ & $\begin{array}{r}3,050 \\
(7.3 \%)\end{array}$ \\
\hline $\begin{array}{l}\text { - With } 6 \text { or More Tires } \\
\text { With Trailer(s) }\end{array}$ & $(0.0 \%)$ & $\begin{array}{r}0 \\
(0.0 \%)\end{array}$ & $\begin{array}{r}18 \\
(0.6 \%)\end{array}$ & $\begin{array}{r}319 \\
(968 \%)\end{array}$ & $\begin{array}{r}0 \\
(0.0 \%)\end{array}$ & $(0.0 \%)$ & $\begin{array}{r}0 \\
(0.0 \%)\end{array}$ & $\begin{array}{r}19 \\
(0.6 \%)\end{array}$ & $\begin{array}{r}355 \\
(a 9 \%)\end{array}$ \\
\hline \multicolumn{10}{|l|}{ Truck-Tractor } \\
\hline - With Trailer(s) & $(0.0 \%)$ & $(0.0 \%)$ & (a) & $\begin{array}{r}0 \\
(0.0 \%)\end{array}$ & $\begin{array}{r}942 \\
(99.5 \%)\end{array}$ & $(7.6 \%)$ & $(0.0 \%)$ & $\begin{array}{r}23 \\
(0.7 \%)\end{array}$ & $\begin{array}{r}966 \\
(23 \%)\end{array}$ \\
\hline - Without Trailer & $\begin{array}{r}0 \\
(0.0 \%)\end{array}$ & $\begin{array}{r}0 \\
(0.0 \%)\end{array}$ & $(0.0 \%)$ & $(0.1 \%)$ & $(0.0 \%)$ & $(90.1 \%)$ & $(0.0 \%)$ & (a) & $\begin{array}{r}18 \\
\text { (a) }\end{array}$ \\
\hline Other & (a) & $(0.0 \%)$ & $(0.0 \%)$ & $\begin{array}{r}0 \\
(0.0 \%)\end{array}$ & (a) & $(0.0 \%)$ & $\begin{array}{r}583 \\
(95.2 \%)\end{array}$ & $\begin{array}{r}23 \\
(0.7 \%)\end{array}$ & $\begin{array}{r}610 \\
(1.5 \%)\end{array}$ \\
\hline Unknown & $\begin{array}{r}257 \\
(0.8 \%)\end{array}$ & $\begin{array}{r}95 \\
(13.6 \%)\end{array}$ & $\begin{array}{r}33 \\
(1.1 \%)\end{array}$ & $(0.3 \%)$ & $(0.4 \%)^{4}$ & $(23 \%)^{a}$ & $(1.2 \%)^{7}$ & $\begin{array}{r}111 \\
(3.5 \%)\end{array}$ & $\begin{array}{r}508 \\
(1.2 \%)\end{array}$ \\
\hline TOTAIS & $\begin{array}{r}33,179 \\
(1000 \%)\end{array}$ & $(1000 \%)$ & $\begin{array}{c}2862 \\
(1000 \%)\end{array}$ & $\begin{array}{r}329 \\
(1000 \%)\end{array}$ & $(100.0 \%)$ & $(1000 \%)$ & $\begin{array}{r}612 \\
(1000 \%)\end{array}$ & $\begin{array}{r}3,128 \\
(100,0 \%)\end{array}$ & $\begin{array}{c}41,768 \\
(100 \%)\end{array}$ \\
\hline
\end{tabular}

(a) Quantity rounded to zero. 


\section{CHAPTER 4}

\section{UNWEIGHTED NUMBERS ON TRIP-SEGMENTS}

- Only 9,974 sample truck operators reported sample day data; the unweighted numbers are counts obtained from that data.

- A daily trip-segment is the section of road traveled between one reported stop and the next reported stop on a truck's sample day.

- Because some of the 9,794 trucks failed to report stop data, there is additional nonresponse and it was decided to present unweighted data in this chapter. 
Table 4.1

Unweighted Number of Daily Trip Segments for Four Year and Older Trucles by Trip-Segment Length and Commodity-Carrying Status: 1990 NTACS

\begin{tabular}{|c|c|c|c|c|c|c|}
\hline \multirow[b]{3}{*}{$\begin{array}{l}\text { Trip-Segment } \\
\text { Length (Miles) }\end{array}$} & \multicolumn{5}{|c|}{ Commodity-Carrying Status } & \multirow[b]{3}{*}{$\frac{\text { TOTALS }}{\text { Trip-Seguesil }}$} \\
\hline & \multicolumn{2}{|c|}{ Commodity-Carrying } & \multicolumn{3}{|c|}{ Non-Commodity-Carrying } & \\
\hline & $\frac{\text { Short Haul }}{\text { Trip-Segments }}$ & $\frac{\text { Long Haul }}{\text { Trip-Segments }}$ & $\frac{\text { Busines: Use }}{\text { Trip-Segments }}$ & $\frac{\text { Pereonal Use }}{\text { Trip-Segments }}$ & $\frac{\text { Not In Use }}{\text { Trip-Segments }}$ & \\
\hline \multirow[t]{2}{*}{ Less Than 10} & 10,454 & 1,549 & 417 & 43 & 28 & 12,491 \\
\hline & $(59.5 \%)$ & $(21.8 \%)$ & $(53.0 \%)$ & $(41.0 \%)$ & $(75.7 \%)$ & (488\%) \\
\hline \multirow[t]{2}{*}{$10-19$} & 2,537 & 614 & 176 & 22 & 1 & 3,350 \\
\hline & $(14.4 \%)$ & $(8.6 \%)$ & $(22.4 \%)$ & $(21.0 \%)$ & $(27 \%)$ & $(13.1 \%)$ \\
\hline \multirow[t]{2}{*}{$20-29$} & 1,614 & 565 & 81 & 24 & 4 & 2,2888 \\
\hline & $(9.2 \%)$ & $(7.9 \%)$ & $(10.3 \%)$ & $(22.9 \%)$ & $(10.8 \%)$ & $(2.9 \times)$ \\
\hline \multirow[t]{2}{*}{$30-39$} & 874 & 513 & 33 & 4 & 1 & 1,425 \\
\hline & $(5.0 \%)$ & $(7.2 \%)$ & $(4.2 \%)$ & $(3.8 \%)$ & $(2.7 \%)$ & $(50 \%)$ \\
\hline \multirow[t]{2}{*}{$40-49$} & 554 & 327 & 18 & 4 & $\mathbf{0}$ & 903 \\
\hline & $(3.2 \%)$ & $(4.6 \%)$ & $(2.3 \%)$ & (3.8\%) & $(0.0 \%)$ & $(3.5 \%)$ \\
\hline \multirow[t]{2}{*}{$50-99$} & 1,066 & 1,120 & 41 & 6 & 1 & 2,234 \\
\hline & $(6.1 \%)$ & (15.8\%) & $(5.2 \%)$ & $(5.7 \%)$ & $(2.7 \%)$ & $(87 \% 5)$ \\
\hline \multirow[t]{2}{*}{$100-149$} & 268 & 701 & 5 & 1 & $\mathbf{0}$ & 975 \\
\hline & $(1.5 \%)$ & $(9.9 \%)$ & $(0.6 \%)$ & $(1.0 \%)$ & $(0.0 \%)$ & $(38 \% 5)$ \\
\hline \multirow[t]{2}{*}{$150-199$} & 100 & 510 & 11 & 0 & 2 & 623 \\
\hline & $(0.6 \%)$ & $(7.2 \%)$ & $(1.4 \%)$ & $(0.0 \%)$ & $(5.4 \%)$ & $(2+x)$ \\
\hline \multirow[t]{2}{*}{$200-399$} & 82 & 832 & 5 & 1 & 0 & 920 \\
\hline & $(0.5 \%)$ & $(11.7 \%)$ & $(0.6 \%)$ & $(1.0 \%)$ & $(0.0 \%)$ & $(3.6 \times)$ \\
\hline \multirow[t]{2}{*}{$400-599$} & 11 & 300 & 0 & $\mathbf{0}$ & $\mathbf{0}$ & 311 \\
\hline & $(0.1 \%)$ & $(4.2 \%)$ & $(0.0 \%)$ & $(0.0 \%)$ & $(0.0 \%)$ & $(1.2 \%)$ \\
\hline \multirow[t]{2}{*}{$600-799$} & 3 & 48 & 0 & 0 & $\mathbf{0}$ & $\mathbf{5 1}$ \\
\hline & (a) & $(0.7 \%)$ & $(0.0 \%)$ & $(0.0 \%)$ & $(0.0 \%)$ & $(a .2 \%)$ \\
\hline \multirow[t]{2}{*}{$800-999$} & 0 & 18 & 0 & 0 & $\mathbf{0}$ & 18 \\
\hline & $(0.0 \%)$ & $(0.3 \%)$ & $(0.0 \%)$ & $(0.0 \%)$ & $(0.0 \%)$ & $(a .1 \%)$ \\
\hline \multirow[t]{2}{*}{1000 and Over } & 0 & 10 & 0 & 0 & $\mathbf{0}$ & 10 \\
\hline & $(0.0 \%)$ & $(0.1 \%)$ & $(0.0 \%)$ & $(0.0 \%)$ & $(0.0 \%)$ & (a) \\
\hline \multirow[t]{2}{*}{ TOTALS } & 17,563 & 7,107 & 787 & 105 & 37 & 25,599 \\
\hline & $(1000 \%)$ & $(1000 x)$ & $(1000 \%)$ & $(1000 x)$ & $(1000 \%)$ & $(1000 \%)$ \\
\hline
\end{tabular}

(a) Quantity rounded to zero. 
Table 4.2

Unweighted Number of Daily Trip Segments for Four Year and Older Trucks by Trip-Segment Length and Truct Type: 1990 NTACS

\begin{tabular}{|c|c|c|c|c|c|c|}
\hline \multirow[b]{2}{*}{$\begin{array}{l}\text { Trip-Segment } \\
\text { Length (Miles) }\end{array}$} & \multicolumn{5}{|c|}{ Truck Type } & \multirow[b]{2}{*}{$\begin{array}{l}\text { Torals } \\
\text { Inipsciment }\end{array}$} \\
\hline & $\frac{\text { Pickup }}{\text { Trip-Segments }}$ & $\frac{\text { Utility }}{\text { Trip-Segments }}$ & $\frac{\text { Small }}{\text { Trip-Segments }}$ & $\frac{\text { Large }}{\text { Trip-Segments }}$ & $\begin{array}{l}\text { Truck-Tractor } \\
\text { Trip-Segments }\end{array}$ & \\
\hline \multirow[t]{2}{*}{ Less Than 10} & 540 & 403 & 3,602 & 3,833 & 4,113 & 12,491 \\
\hline & $(62.6 \%)$ & $(61.6 \%)$ & $(71.2 \%)$ & $(56.6 \%)$ & $(33.6 \%)$ & (428\%) \\
\hline \multirow[t]{2}{*}{$10 \cdot 19$} & 131 & 113 & 572 & 1,123 & 1,411 & 3,350 \\
\hline & (15.2\%) & $(17.3 \%)$ & (11.3\%) & $(16.6 \%)$ & (11.5\%) & $(13.1 \%)$ \\
\hline \multirow[t]{2}{*}{$20-29$} & 79 & 60 & 375 & 612 & 1,162 & 2,283 \\
\hline & $(9.2 \%)$ & $(9.2 \%)$ & $(7.4 \%)$ & $(9.0 \%)$ & (9.5\%) & $(89 \times)$ \\
\hline \multirow[t]{2}{*}{$30 \cdot 39$} & 43 & 33 & 171 & 364 & 814 & 1,425 \\
\hline & $(5.0 \%)$ & $(5.0 \%)$ & $(3.4 \%)$ & $(5.4 \%)$ & $(6.6 \%)$ & $(5.6 \%)$ \\
\hline \multirow[t]{2}{*}{$40-49$} & 24 & 18 & 91 & 219 & 551 & $\sin$ \\
\hline & $(28 \%)$ & $(28 \%)$ & $(1.8 \%)$ & $(3.2 \%)$ & (4.5\%) & (3.5\%) \\
\hline \multirow[t]{2}{*}{$50-99$} & 36 & 22 & 183 & 377 & 1,616 & 2,234 \\
\hline & $(4.2 \%)$ & $(3.4 \%)$ & $(3.6 \%)$ & $(5.6 \%)$ & $(13.2 \%)$ & $(27 \%)$ \\
\hline \multirow[t]{2}{*}{$100 \cdot 149$} & 4 & 2 & 37 & 111 & 821 & 975 \\
\hline & (a.5\%) & $(0.3 \%)$ & $(0.7 \%)$ & $(1.6 \%)$ & $(6.7 \%)$ & (388\%) \\
\hline \multirow[t]{2}{*}{$150-199$} & 3 & 1 & 19 & 42 & 558 & 123 \\
\hline & $(0.3 \%)$ & $(0.2 \%)$ & $(0.4 \%)$ & $(0.6 \%)$ & $(4.6 \%)$ & $(24 \times 3)$ \\
\hline \multirow[t]{2}{*}{$200-399$} & 2 & 2 & 9 & 79 & 828 & 200 \\
\hline & $(0.2 \%)$ & $(0.3 \%)$ & $(0.2 \%)$ & $(1.2 \%)$ & $(6.8 \%)$ & $(36 x)$ \\
\hline \multirow[t]{2}{*}{$400 \cdot 599$} & $\mathbf{0}$ & 0 & 1 & 17 & 293 & 311 \\
\hline & $(0.0 \%)$ & $(0.0 \%)$ & (a) & $(0.3 \%)$ & $(24 \%)$ & $(1.2 \% 5)$ \\
\hline \multirow[t]{2}{*}{$600-799$} & o & $\mathbf{0}$ & 1 & 1 & 49 & 51 \\
\hline & $(0.0 \%)$ & $(0.0 \%)$ & (a) & (a) & $(0.4 \%)$ & $(a, 2 \times 5)$ \\
\hline \multirow[t]{2}{*}{$800-999$} & 0 & 0 & 0 & 0 & 18 & 18 \\
\hline & $(0.0 \%)$ & $(0.0 \%)$ & $(0.0 \%)$ & $(0.0 \%)$ & $(0.1 \%)$ & (a.1\%) \\
\hline \multirow[t]{2}{*}{1000 and Over } & $\mathbf{0}$ & 0 & 0 & $\mathbf{0}$ & 10 & 10 \\
\hline & $(0.0 \%)$ & $(0.0 \%)$ & $(0.0 \%)$ & $(0.0 \%)$ & $(0.1 \%)$ & (a) \\
\hline \multirow[t]{2}{*}{ TOTALS } & 862 & 654 & 5,061 & 6,778 & $12,2 \mathrm{M}$ & 25,599 \\
\hline & $(1000 x)$ & $(1000 x)$ & $(1000 \times)$ & $(1000 x)$ & $(1000 x)$ & $(1000 \%)$ \\
\hline
\end{tabular}

(a) Quantity rounded to zero. 
Table 4.3

Unweighted Summary Statistics for Length of Trip Segments for Four Year and Older Trucks by Truck Description from 1990 NTACS and Major Use from 1987 TIUS

\begin{tabular}{|c|c|c|c|c|c|c|c|c|c|}
\hline & \multicolumn{8}{|c|}{ Truck Description } & \multirow[b]{3}{*}{ Total } \\
\hline & \multicolumn{2}{|c|}{$\begin{array}{c}\text { Straight Truck } \\
\text { with } 4 \text { Tires }\end{array}$} & \multicolumn{2}{|c|}{$\begin{array}{c}\text { Straight Truck } \\
\text { with } 6 \text { Tires }\end{array}$} & \multicolumn{2}{|c|}{ Truck-Tractor } & \multirow[b]{2}{*}{ Other } & \multirow[b]{2}{*}{ Unknown } & \\
\hline & $\begin{array}{c}\text { without } \\
\text { trailer }\end{array}$ & $\begin{array}{l}\text { with } \\
\text { trailer(s) }\end{array}$ & $\begin{array}{l}\text { without } \\
\text { trailer }\end{array}$ & $\begin{array}{l}\text { with } \\
\text { trailer(s) }\end{array}$ & $\begin{array}{l}\text { with } \\
\text { trailer(s) }\end{array}$ & $\begin{array}{l}\text { without } \\
\text { trailer }\end{array}$ & & & \\
\hline & & & & Agr & tural Servic & & & & \\
\hline No. of Trip Segments & 319.00 & 41.00 & $1,519.00$ & 116.00 & 700.00 & 7.00 & 7.00 & 18.00 & $2,727.00$ \\
\hline Average (miles) & 11.46 & 20.44 & 15.91 & 36.35 & 78.35 & 50.00 & 15.00 & 10.17 & 3240 \\
\hline Minimum (miles) & 1.00 & 1.00 & 1.00 & 1.00 & 1.00 & 5.00 & 7.00 & 1.00 & 1.00 \\
\hline \multirow[t]{2}{*}{ Maximum (miles) } & 120.00 & 168.00 & 300.00 & 275.00 & 850.00 & 200.00 & 30.00 & 41.00 & 850.00 \\
\hline & \multicolumn{9}{|c|}{ Forestry or Lumbering Activities } \\
\hline No. of Trip Segments & 12.00 & 2.00 & 219.00 & 54.00 & 566.00 & 5.00 & 5.00 & 0 & 863.00 \\
\hline Average (miles) & 13.92 & 14.50 & 21.31 & 49.13 & 53.42 & 52.80 & 94.40 & $\cdot$ & 44.60 \\
\hline Minimum (miles) & 1.00 & 14.00 & 1.00 & 1.00 & 1.00 & 5.00 & 6.00 & - & 1.00 \\
\hline \multirow[t]{2}{*}{ Maximum (miles) } & 33.00 & 15.00 & 183.00 & 170.00 & 500.00 & 105.00 & 200.00 & $\cdot$ & 500.00 \\
\hline & \multicolumn{9}{|c|}{ Construction Work } \\
\hline No. of Trip Segments & 175.00 & 16.00 & $1,676.00$ & 252.00 & 992.00 & 19.00 & 72.00 & 19.00 & $3,221.00$ \\
\hline Average (miles) & 14.78 & 19.94 & . 13.58 & 22.29 & 38.54 & 10.05 & 12.11 & 21.00 & 204 \\
\hline Minimum (miles) & 1.00 & 2.00 & 1.00 & 1.00 & 1.00 & 1.00 & 1.00 & 5.00 & 1.00 \\
\hline \multirow[t]{2}{*}{ Maximum (miles) } & 60.00 & 60.00 & 325.00 & 243.00 & 943.00 & 22.00 & 48.00 & 115.00 & 943.00 \\
\hline & \multicolumn{9}{|c|}{ Contractor Activities or Special Trades } \\
\hline No. of Trip Segments & 388.00 & 1.00 & 489.00 & 41.00 & 116.00 & 4.00 & 6.00 & 4.00 & $1,049.00$ \\
\hline Average (miles) & 12.99 & 10.00 & 15.82 & 24.39 & 56.71 & 112.50 & 39.67 & 14.00 & 20.12 \\
\hline Minimum (miles) & 1.00 & 10.00 & 1.00 & 1.00 & 2.00 & 35.00 & 10.00 & 3.00 & 1.00 \\
\hline \multirow[t]{2}{*}{ Maximum (miles) } & 95.00 & 10.00 & 650.00 & 350.00 & $1,400.00$ & 300.00 & 66.00 & 25.00 & $1,400.00$ \\
\hline & \multicolumn{9}{|c|}{ Manufacturing, Refining, or Processing Activities } \\
\hline No. of Trip Segments & 145.00 & 9.00 & $\mathbf{7 2 1 . 0 0}$ & 59.00 & 990.00 & 0 & 13.00 & 2.00 & 1939.00 \\
\hline Average (milex) & 18.46 & 38.89 & 13.82 & 39.71 & 89.81 & $\cdot$ & 10.23 & 3.00 & 53.83 \\
\hline Minimum (miles) & 1.00 & 1.00 & 1.00 & 1.00 & 1.00 & - & 1.00 & 3.00 & 1.00 \\
\hline \multirow[t]{2}{*}{ Maximum (miles) } & 215.00 & 175.00 & 420.00 & 450.00 & $1,488.00$ & - & 50.00 & 3.00 & $1,488.00$ \\
\hline & \multicolumn{9}{|c|}{ Wholesale Trade } \\
\hline No. of Trip Segments & 443.00 & 12.00 & $2,360.00$ & 87.00 & $1,616.00$ & 2.00 & 27.00 & 14.00 & $4,561.00$ \\
\hline Average (miles) & 9.13 & 10.83 & 12.49 & 56.86 & 49.55 & 18.00 & 13.81 & 17.21 & 26.16 \\
\hline Minimum (miles) & 1.00 & 1.00 & 1.00 & 1.00 & 1.00 & 1.00 & 1.00 & 1.00 & 1.00 \\
\hline Maximum (miles) & 115.00 & 31.00 & 400.00 & 435.00 & 700.00 & 35.00 & 35.00 & 100.00 & 7000 \\
\hline
\end{tabular}


Table 4.3

Unweighted Summary Statistics for Length of Trip Segments for Four Year and Older Trucks by Truck Description from 1990 NTACS and Major Use from 1987 TIUS (Continued)

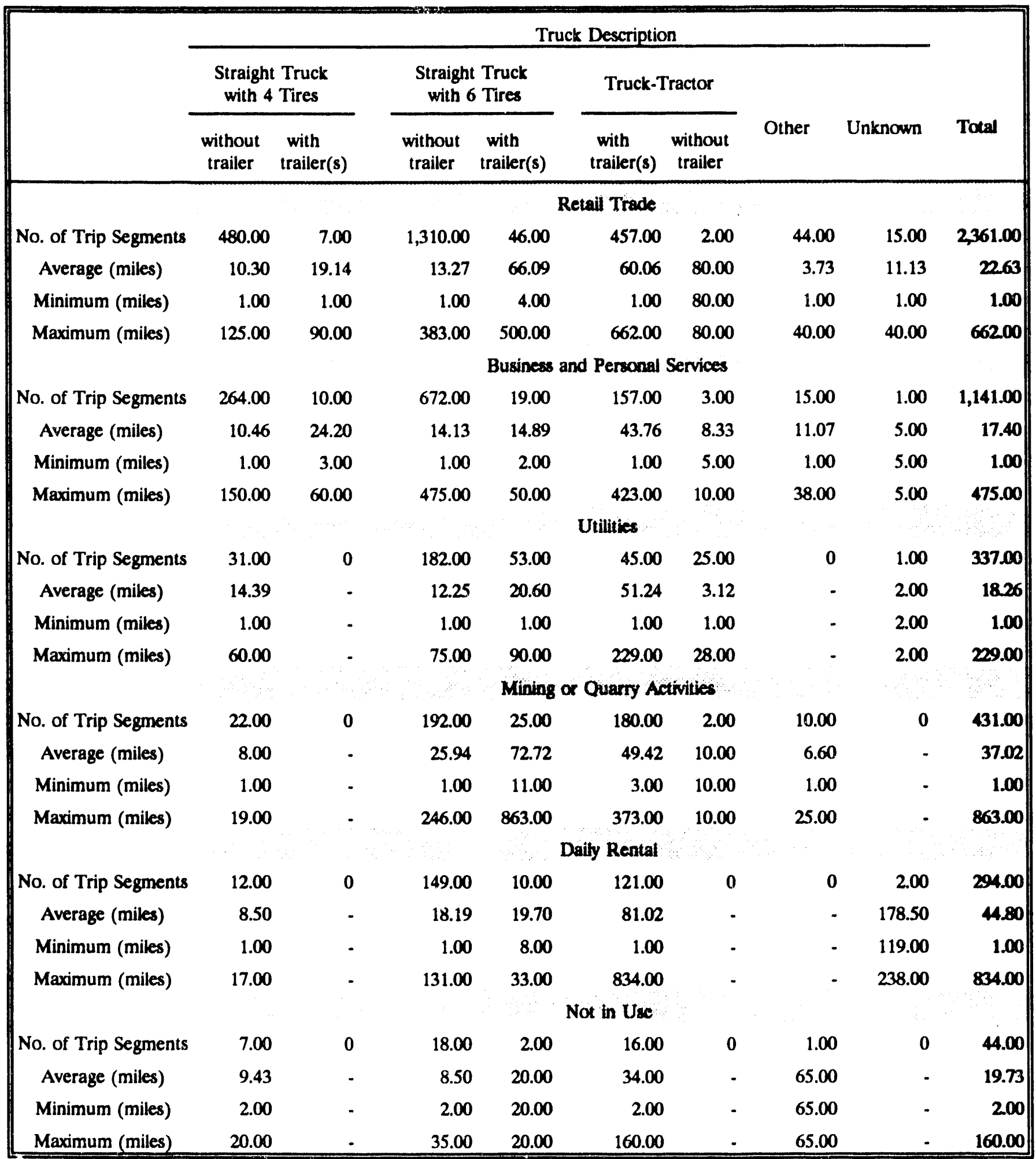


Table 4.3

Unweighted Summary Statistics for Length of Trip Segments for Four Year and Older Tructs by Truck Description from 1990 NTACS and Major Use from 1987 TIUS (Continued)

\begin{tabular}{|c|c|c|c|c|c|c|c|c|c|}
\hline & \multicolumn{8}{|c|}{ Truck Description } & \multirow[b]{3}{*}{ Totsl } \\
\hline & \multicolumn{2}{|c|}{$\begin{array}{c}\text { Straight Truck } \\
\text { with } 4 \text { Tires }\end{array}$} & \multicolumn{2}{|c|}{$\begin{array}{c}\text { Straight Truck } \\
\text { with } 6 \text { Tires }\end{array}$} & \multicolumn{2}{|c|}{ Truck-Tractor } & \multirow[b]{2}{*}{ Other } & \multirow[b]{2}{*}{ Unknown } & \\
\hline & $\begin{array}{l}\text { without } \\
\text { trailer }\end{array}$ & $\begin{array}{l}\text { with } \\
\text { trailer(s) }\end{array}$ & $\begin{array}{l}\text { without } \\
\text { trailer }\end{array}$ & $\begin{array}{l}\text { with } \\
\text { trailer(s) }\end{array}$ & $\begin{array}{l}\text { with } \\
\text { trailer(s) }\end{array}$ & $\begin{array}{l}\text { without } \\
\text { trailer }\end{array}$ & & & \\
\hline & \multicolumn{9}{|c|}{ For Hire Transportation } \\
\hline No. of Trip Segments & 88.00 & 5.00 & 796.00 & 232.00 & $5,200.00$ & 19.00 & 42.00 & 15.00 & $6,397.00$ \\
\hline Average (miles) & 13.45 & 57.80 & 17.38 & 40.24 & 91.19 & 113.84 & 23.64 & 59.93 & 7861 \\
\hline Minimum (miles) & 1.00 & 7.00 & 1.00 & 1.00 & 1.00 & 10.00 & 1.00 & 1.00 & 1.00 \\
\hline \multirow[t]{2}{*}{ Maximum (miles) } & 241.00 & 100.00 & 420.00 & 725.00 & $1,300.00$ & 600.00 & 556.00 & 350.00 & $1,300.00$ \\
\hline & \multicolumn{9}{|c|}{ One Way Rental } \\
\hline No. of Trip Segments & 0 & 0 & 10.00 & 0 & 0 & 0 & 0 & 0 & 10.00 \\
\hline Average (miles) & - & - & 20.80 & - & - & - & - & - & 2080 \\
\hline Minimum (miles) & - & - & 5.00 & - & $\cdot$ & - & - & - & 5.00 \\
\hline \multirow[t]{2}{*}{ Maximum (miles) } & - & - & 50.00 & - & - & $\cdot$ & - & - & 50.00 \\
\hline & \multicolumn{9}{|c|}{ Personal Transportation } \\
\hline No. of Trip Segments & 78.00 & 2.00 & 10.00 & 5.00 & 0 & 0 & 2.00 & 0 & 97.00 \\
\hline Average (miles) & 18.58 & 45.00 & 32.50 & 27.60 & - & - & 10.00 & - & 20.85 \\
\hline Minimum (miles) & 1.00 & 45.00 & 18.00 & 26.00 & $\cdot$ & - & 10.00 & - & 1.00 \\
\hline \multirow[t]{2}{*}{ Maximum (miles) } & 241.00 & 45.00 & 129.00 & 30.00 & - & $\cdot$ & 10.00 & - & 241.00 \\
\hline & \multicolumn{9}{|c|}{ Unknown } \\
\hline No. of Trip Segments & 12.00 & 0 & 45.00 & 13.00 & 57.00 & 0 & 0 & 0 & 127.00 \\
\hline Average (miles) & 13.83 & - & 19.87 & 150.31 & 88.05 & - & - & $\cdot$ & 63.25 \\
\hline Minimum (miles) & 5.00 & - & 2.00 & 9.00 & 1.00 & - & - & - & 1.00 \\
\hline \multirow[t]{2}{*}{ Maximum (miles) } & 17.00 & - & 150.00 & 795.00 & 500.00 & - & - & $\cdot$ & 795.00 \\
\hline & & & & & Totel & & & & \\
\hline Na of Trip Segments & $2,476.00$ & 105.00 & 10,36800 & $1,014.00$ & $11,213.00$ & 88.00 & 244.00 & 91.00 & $25,599.00$ \\
\hline Akerage (miles) & 11.90 & $\mathbf{2 3 . 1 5}$ & 14.56 & 38.14 & 74.38 & 4247 & 15.03 & 25.44 & 41.61 \\
\hline Minimum (miles) & 1.00 & 1.00 & 1.00 & 1.00 & 1.00 & 1.00 & 1.00 & 1.00 & 1.00 \\
\hline Marimum (miles). & 241.00 & 175.00 & 650.00 & 863.00 & $1,488.00$ & 600.00 & 556.00 & 350.00 & $1,488.00$ \\
\hline
\end{tabular}


Table 4.4

Unweighted Number of Daily Four Year and Older Trucls

by Number of Daily Pickup/Delivery Stops and Commodity-Carrying Status: 1990 NTACS

\begin{tabular}{|c|c|c|c|c|c|c|}
\hline \multirow{3}{*}{$\begin{array}{l}\text { Number of Daily } \\
\text { Pickup/Delivery } \\
\text { Stope }\end{array}$} & \multicolumn{5}{|c|}{ Commodity-Carrying Status } & \multirow[b]{3}{*}{$\frac{\text { TOTAls }}{\text { rructs }}$} \\
\hline & \multicolumn{2}{|c|}{ Commodity-Carrying } & \multicolumn{3}{|c|}{ Non-Commodity-Carrying } & \\
\hline & $\frac{\text { Short Haul }}{\text { Irucks }}$ & $\frac{\text { Lone Haul }}{\text { Irucka }}$ & $\frac{\text { Businese Use }}{\text { Irucks }}$ & $\frac{\text { Pereonal Use }}{\text { Irucks }}$ & $\frac{\text { Not In Use }}{\text { Trucks }}$ & \\
\hline \multirow[t]{2}{*}{$\mathbf{0}$} & 539 & 541 & 289 & 166 & 6 & 1,541 \\
\hline & $(9.9 \%)$ & $(19.2 \%)$ & $(45.6 \%)$ & $(19.6 \%)$ & $(20.7 \%)$ & $(15.7 \%)$ \\
\hline \multirow[t]{2}{*}{1} & 1,257 & 846 & 172 & 394 & 8 & 2,677 \\
\hline & $(23.0 \%)$ & $(30.1 \%)$ & $(27.1 \%)$ & $(46.5 \%)$ & $(27.6 \%)$ & $(27.3 \%)$ \\
\hline \multirow[t]{2}{*}{2} & 1,256 & 663 & 108 & 204 & 7 & 2,2038 \\
\hline & $(23.0 \%)$ & $(23.6 \%)$ & $(17.0 \%)$ & $(24.1 \%)$ & $(24.1 \%)$ & $(229 x)$ \\
\hline \multirow[t]{2}{*}{3} & 652 & 253 & 16 & 37 & 6 & 964 \\
\hline & (11.9\%) & $(9.0 \%)$ & $(25 \%)$ & $(4.4 \%)$ & $(20.7 \%)$ & $(9.8 \%)$ \\
\hline \multirow[t]{2}{*}{4} & 468 & 207 & 28 & 46 & 1 & 750 \\
\hline & $(8.6 \%)$ & $(7.4 \%)$ & $(4.4 \%)$ & $(5.4 \%)$ & $(3.4 \%)$ & $(7.7 \%)$ \\
\hline \multirow[t]{2}{*}{5} & 299 & 131 & 8 & 0 & $\mathbf{0}$ & 438 \\
\hline & $(5.5 \%)$ & $(4.7 \%)$ & (1.3\%) & $(0.0 \%)$ & $(0.0 \%)$ & (4.5\%) \\
\hline \multirow[t]{2}{*}{6} & 250 & 69 & 6 & 0 & $\mathbf{0}$ & 325 \\
\hline & $(4.6 \%)$ & $(25 \%)$ & $(0.9 \%)$ & $(0.0 \%)$ & $(0.0 \%)$ & (3.3\%) \\
\hline \multirow[t]{2}{*}{7} & 120 & 21 & 0 & 0 & 1 & 142 \\
\hline & $(2.2 \%)$ & $(0.7 \%)$ & $(0.0 \%)$ & $(0.0 \%)$ & $(3.4 \%)$ & $(1.45)$ \\
\hline \multirow[t]{2}{*}{8} & 105 & 27 & 6 & 0 & o & 138 \\
\hline & $(1.9 \%)$ & $(1.0 \%)$ & $(0.9 \%)$ & $(0.0 \%)$ & $(0.0 \%)$ & $(1.4 \times)$ \\
\hline \multirow[t]{2}{*}{9} & 57 & 11 & 0 & 0 & 0 & 68 \\
\hline & $(1.0 \%)$ & $(0.4 \%)$ & $(0.0 \%)$ & $(0.0 \%)$ & $(0.0 \%)$ & $(a 7 \pi)$ \\
\hline \multirow[t]{2}{*}{10} & 98 & 10 & 0 & 0 & 0 & 108 \\
\hline & $(1.8 \%)$ & $(0.4 \%)$ & $(0.0 \%)$ & $(0.0 \%)$ & $(0.0 \%)$ & (1.1\%) \\
\hline \multirow[t]{2}{*}{$11-15$} & 190 & 19 & 1 & 0 & 0 & 210 \\
\hline & (3.5\%) & $(0.7 \%)$ & $(0.2 \%)$ & $(0.0 \%)$ & $(0.0 \%)$ & (21\%) \\
\hline \multirow[t]{2}{*}{$16-20$} & 105 & 11 & 0 & $\mathbf{0}$ & $\mathbf{0}$ & 116 \\
\hline & $(1.9 \%)$ & $(0.4 \%)$ & $(0.0 \%)$ & $(0.0 \%)$ & $(0.0 \%)$ & $(1.2 \%)$ \\
\hline \multirow[t]{2}{*}{$21 \cdot 25$} & 48 & 3 & $\mathbf{0}$ & 0 & 0 & 51 \\
\hline & $(0.9 \%)$ & $(0.1 \%)$ & $(0.0 \%)$ & $(0.0 \%)$ & $(0.0 \%)$ & (as\%) \\
\hline \multirow[t]{2}{*}{25 and Over } & 27 & 1 & $\mathbf{0}$ & 0 & 0 & 28 \\
\hline & $(0.5 \%)$ & (a) & $(0.0 \%)$ & $(0.0 \%)$ & $(0.0 \%)$ & (a.3\%) \\
\hline \multirow[t]{2}{*}{ TOTALS } & 5,471 & 28813 & 634 & 847 & 20 & 9,794 \\
\hline & $(1000 \%)$ & (1000x) & (10aron) & $(10 a 0 \%)$ & $(1000 \%)$ & $(1000 x)$ \\
\hline
\end{tabular}

(a) Quantity rounded to zero. 
Table 4.5

Unweighted Number of Daily Four Year and Older Trucks by Number of Daily Pickup/Delivery Stops and Daily Major Use: 1990 NTACS

\begin{tabular}{|c|c|c|c|c|c|c|}
\hline \multirow[b]{2}{*}{$\begin{array}{l}\text { Number of Daily } \\
\text { Pickup/Delivery } \\
\text { Stops }\end{array}$} & \multicolumn{5}{|c|}{ Daily Major Use } & \multirow[b]{2}{*}{$\frac{\text { TOTALS }}{\text { Truats }}$} \\
\hline & $\begin{array}{c}\text { Personal } \\
\text { Transportation } \\
\text { Trucks }\end{array}$ & $\begin{array}{c}\text { Contract } \\
\text { Carrier } \\
\text { Trucks }\end{array}$ & $\begin{array}{l}\text { Common } \\
\text { Carrier } \\
\text { Trucks }\end{array}$ & $\begin{array}{c}\text { Other } \\
\text { Businese Use } \\
\text { Trucks }\end{array}$ & $\frac{\text { Unknown }}{\text { Truckes }}$ & \\
\hline \multirow[t]{2}{*}{0} & 301 & 171 & 347 & 664 & 58 & 1,541 \\
\hline & $(17.8 \%)$ & (14.3\%) & (15.7\%) & $(16.4 \%)$ & $(9.1 \%)$ & $(157 \times 3)$ \\
\hline \multirow[t]{2}{*}{1} & 629 & 300 & 556 & 1,065 & 127 & 2677 \\
\hline & $(37.2 \%)$ & $(25.0 \%)$ & $(25.2 \%)$ & $(26.2 \%)$ & $(20.0 \%)$ & (27.3\%) \\
\hline \multirow[t]{2}{*}{2} & 450 & 276 & 478 & 863 & 171 & 2,238 \\
\hline & $(26.6 \%)$ & $(23.0 \%)$ & $(21.6 \%)$ & $(21.3 \%)$ & $(27.0 \%)$ & $(229 \%)$ \\
\hline \multirow[t]{2}{*}{3} & 151 & 99 & 172 & 389 & 153 & 964 \\
\hline & $(8.9 \%)$ & $(8.3 \%)$ & $(7.8 \%)$ & (9.6\%) & $(24.1 \%)$ & $(9.8 \%)$ \\
\hline \multirow[t]{2}{*}{4} & 78 & 128 & 185 & 295 & 64 & 750 \\
\hline & (4.6\%) & $(10.7 \%)$ & (8.4\%) & $(7.3 \%)$ & $(10.1 \%)$ & (7.7\%) \\
\hline \multirow[t]{2}{*}{5} & 41 & 52 & 113 & 205 & 27 & 438 \\
\hline & $(24 \%)$ & $(4.3 \%)$ & (5.1\%) & $(5.0 \%)$ & $(4.3 \%)$ & (4.5\%) \\
\hline \multirow[t]{2}{*}{6} & 15 & 55 & 91 & 150 & 14 & 325 \\
\hline & $(0.9 \%)$ & $(4.6 \%)$ & $(4.1 \%)$ & $(3.7 \%)$ & $(2.2 \%)$ & $(3.3 \times 5)$ \\
\hline \multirow[t]{2}{*}{7} & 12 & 21 & 34 & 71 & 4 & 142 \\
\hline & $(0.7 \%)$ & $(1.7 \%)$ & (1.5\%) & $(1.7 \%)$ & $(0.6 \%)$ & $(1.4 x)$ \\
\hline \multirow[t]{2}{*}{8} & 7 & 32 & 33 & 62 & 4 & 138 \\
\hline & $(0.4 \%)$ & $(27 \%)$ & (1.5\%) & (1.5\%) & $(0.6 \%)$ & $(1.4 \times)$ \\
\hline \multirow[t]{2}{*}{9} & 0 & 6 & 24 & 37 & 1 & 68 \\
\hline & $(0.0 \%)$ & $(0.5 \%)$ & $(1.1 \%)$ & $(0.9 \%)$ & $(0.2 \%)$ & $(a 723)$ \\
\hline \multirow[t]{2}{*}{10} & 0 & 12 & 35 & 61 & $\mathbf{0}$ & 108 \\
\hline & $(0.0 \%)$ & $(1.0 \%)$ & (1.6\%) & $(1.5 \%)$ & $(0.0 \%)$ & (1.1\%) \\
\hline \multirow[t]{2}{*}{$11-15$} & 4 & 27 & 66 & 108 & 5 & 210 \\
\hline & $(0.2 \%)$ & $(23 \%)$ & $(3.0 \%)$ & $(2.7 \%)$ & $(0.8 \%)$ & (21\%) \\
\hline \multirow[t]{2}{*}{$16 \cdot 20$} & 1 & 11 & 49 & 51 & 4 & 116 \\
\hline & $(0.1 \%)$ & $(0.9 \%)$ & $(22 \%)$ & $(1.3 \%)$ & $(0.6 \%)$ & $(1.2 \times 3)$ \\
\hline \multirow[t]{2}{*}{$21 \cdot 25$} & 1 & 3 & 20 & 25 & 2 & 51 \\
\hline & $(0.1 \%)$ & $(0.3 \%)$ & $(0.9 \%)$ & $(0.6 \%)$ & $(0.3 \%)$ & (as\%) \\
\hline \multirow[t]{2}{*}{25 and Over } & $\mathbf{0}$ & 7 & 7 & 14 & $\mathbf{0}$ & 28 \\
\hline & $(0.0 \%)$ & $(0.6 \%)$ & $(0.3 \%)$ & $(0.3 \%)$ & $(0.0 \%)$ & (a.3\%) \\
\hline \multirow[t]{2}{*}{ TOTALS } & 1,690 & 1,200 & 2,210 & 4,060 & 634 & 9,794 \\
\hline & (10aos) & (10nox) & (10acx) & $(1000 x)$ & $(102 \times 1 \times 5)$ & (1000\%) \\
\hline
\end{tabular}




\section{CHAPTER 5}

\section{ADJUSTED ESTIMATES OF THE 1990 NTACS TRUCK POPULATION}

- ORNL adjusted the 1990 NTACS population to include estimates of the post-NTACS trucks (less than four years old). 
To provide estimates for the U.S. 1990 population of trucks (regions $A$ and $C$ of Figure 1.1), ORNL investigated several variations of an adjustment technique. The best of those variations, as judged by statistical methods used by ORNL research staff members, produced the estimates presented in Table 5.1, which is to be viewed relative to Table 1.1.

What follows is a brief outline of steps taken to produce the adjusted estimates by producing two adjusted data items:

XPFD1(adj) and ARMIL (adj).

The variation used makes it easy to produce adjusted tables and provides consistent adjusted totals among adjusted tables. The adjustment methodology makes use of

(1) the data items XPFD1 and ARMIL included on the 1990 NTACS Public Use File,

(2) selected data items included on the 1987 TIUS User's Tape, and

(3) R. L. Polk truck population data for 1987 and 1990.

\section{Production of XPFD1(adj)}

Step 1. For either the 1987 TIUS sample (or the 1990 NTACS sample), trucks were stratified into nine strata according to commodity-carrying status and truck type as follows:

\begin{tabular}{|l|l|l|l|}
\hline \multirow{2}{*}{ Truck Type } & \multicolumn{3}{|c|}{ Commodity-Carrying Status } \\
\cline { 2 - 3 } & \multicolumn{2}{|c|}{ Commodity-Carrying } & \multirow{2}{*}{ Non-Commodity Carrying } \\
\cline { 2 - 4 } & Short-Haul & Long-Haul & \\
\hline $\begin{array}{l}\text { Pickup } \\
\text { Utility } \\
\text { Small Truck }\end{array}$ & Stratum 1 & Stratum 2 & Stratum 3 \\
\hline Large Truck & Stratum 4 & Stratum 5 & Stratum 6 \\
\hline Truck-Tractor & Stratum 7 & Stratum 8 & Stratum 9 \\
\hline
\end{tabular}

The age of a truck was represented by $t=1,2,3,4,5,6,7,8,9,10$. Trucks with $t>10$ were not considered in this step but were considered in steps 7-9. 
Step 2. Using the 1987 TIUS Tape and for each value of $t(=1,2,3,4,5,6,7,8,9,10)$, the weighted distribution of the trucks over the nine strata was determined. There were ten different distributions.

Step 3. Using the 1990 NTACS Public Use File and for each value of $t(=4,5,6,7,8,9$, $10)$, the weighted distribution of the trucks over the nine strata was determined. There were seven different distributions.

Step 4. For each $t(=4,5,6,7,8,9,10)$, the distribution from the 1987 TIUS was compared with the corresponding distribution from the 1990 NTACS. There were seven different comparisons.

Step 5. Using 1987 TIUS data and 1987 and 1990 Polk data to adjust for trucks excluded from TIUS, estimates of the number of 1 year old, 2 year old, and 3 year old trucks operating in 1990 were produced as follows:

1990 Estimate $(t$ yr old $)=\frac{\text { TIUS } 1987 \text { Estimate }(t)}{\text { Polk } 1987 \text { Estimate }(t)}$ Polk 1990 Estimate $(t)$

yielding

\begin{tabular}{ll}
\hline$t$ & 1990 Estimate $(t$ yr old) \\
\hline 1 year olds & $2,853,162$ trucks \\
2 year olds & $4,861,769$ trucks \\
3 year olds & $4,629,739$ trucks \\
\hline
\end{tabular}

Step 6. Using the 1987 TIUS distribution of $t$ year old trucks, the 1990 estimate was allocated among the nine strata. This was done three separate times.

Step 7. For each stratum, the estimated number of trucks was obtained as NTACS (all yrs $)=\operatorname{NTACS~}\left(4^{+} \mathrm{yrs}\right)+(1 \mathrm{yr}$ alloc $)+(2 \mathrm{yr}$ alloc $)+(3 \mathrm{yr}$ alloc $)$.

Step 8. For each NTACS sample truck in a given stratum, its adjusted expansion factor was obtained as

$$
X P F D 1(a d j)=\frac{N T A C S(a l l \text { yrs) }}{N T A C S\left(4^{*} \text { yrs }\right)} X P F D 1 .
$$

Step 9. The variable XPFD1(adj) was used to produce all adjusted estimates of the number of trucks in Table 5.1. 


\section{Production of ARMII(adj)}

Step 10. Using the 1987 TIUS Tape for each stratum and for each value of $t(=1,2,3,4$, $5,6,7,8,9,10)$ the (weighted) average VMT per truck was determined. Within each stratum, 10 (weighted) average VMTs were obtained.

Step 11. Using the 1990 NTACS File for each stratum and for each value of $t(=4,5,6$, $7,8,9,10$ ), the (weighted) average VMT per truck was determined. Within each stratum, 7 (weighted) average VMTs were obtained. Note that for NTACS, we did not have the (weighted) averages for $\mathrm{t}=1,2$, nor 3 .

Step 12. Within each stratum and using the averages from steps 10 and 11 for $t=5,6,7$, $8,9,10$, a regression model to predict the 3 unknown averages for NTACS for $t=1,2$, and 3 was used.

Step 13. Within each stratum, an average VMT per truck was predicted separately for 1 year olds, for 2 year olds, and for 3 year olds.

Step 14. Within each stratum, a total adjusted estimate of VMT was produced as:

$$
\begin{aligned}
V M T(N T A C S, a l l)= & V M T(N T A C S, 4+y r s)+V M T(N T A C S, 1 y r) \\
& +V M T(N T A C S, 2 y r)+V M T(N T A C S, 3 y r)
\end{aligned}
$$

Step 15. For each NTACS sample truck in a given stratum, its adjusted ARMIL was obtained as:

$$
A R M I L(\text { adj })=\frac{V M T(N T A C S, a l l)}{V M T(N T A C S, 4+)} A R M I L
$$

Thus each NTACS sample truck's annual reported mileage was adjusted to include its own mileage plus a share of the estimated mileage of similar trucks' ages $t=1$, 2 , and 3 years old.

Step 16. The variable ARMIL(adj) along with the variable XPFD1 was used to produce all adjusted estimates of the annual truck miles in Table 5.1.

In summary, we have

\begin{tabular}{|c|c|c|}
\hline Estimate & Trucks & VMT (miles) \\
\hline Four Year and Older Trucks in 1990 & $41,768,000$ & $440,174,000,000$ \\
All Trucks in 1990 (adjusted) & $54,112,000$ & $652,147,000,000$ \\
\hline
\end{tabular}


Table 5.1

Number (Adjusted to Include Less than Four Year Old) of Tructes and (Adjusted) Associated Annual Vehicle Miles Traveled (VMT)

by Census-Region, Commodity-Carrying Status, and Truct Type: 1990 NTACS

(Trucks in Thousands/Annual VMT in Millions)

\begin{tabular}{|c|c|c|c|c|c|c|c|c|c|c|c|c|}
\hline \multirow[b]{3}{*}{$\begin{array}{l}\text { Commodity } \\
\text { Carrying } \\
\text { Status }\end{array}$} & \multicolumn{10}{|c|}{ Truck Type } & \multirow{2}{*}{\multicolumn{2}{|c|}{ TOTALS }} \\
\hline & \multicolumn{2}{|c|}{ Pickup } & \multicolumn{2}{|c|}{ Utility } & \multicolumn{2}{|c|}{ Small } & \multicolumn{2}{|c|}{ Large } & \multicolumn{2}{|c|}{ Truck-Tractor } & & \\
\hline & Trucks & Annual & Trucies & Annual & Trucks & Annual & Trucks & $\underset{\mathrm{VMT}}{\text { Annual }}$ & Trucks & Annual & Truch & $\begin{array}{l}\text { Annun } \\
\text { VMT }\end{array}$ \\
\hline \multicolumn{13}{|l|}{ Commodity-Carrying } \\
\hline -Shon Haul & 411 & 5,558 & 372 & 4,975 & 360 & 4,566 & 200 & 3,780 & 104 & 3,344 & $1,4 M$ & 2020 \\
\hline -Long Haul & 5 & 28 & 24 & 1,042 & 11 & 145 & 15 & 725 & 66 & 4,660 & 120 & 6,600 \\
\hline \multicolumn{13}{|l|}{ Non-Commodity-Carrying } \\
\hline -Busines Use & 575 & 7,990 & 551 & 9,343 & 139 & 876 & 28 & 346 & 7 & 129 & 1,209 & 18,683 \\
\hline -Personal Use & 2,522 & 19,656 & 1,670 & 17,354 & 45 & 189 & 0 & 0 & $\mathbf{a}$ & $\mathbf{a}$ & 4,237 & 37,199 \\
\hline -Not in Use & 0 & 0 & 0 & 0 & $\mathbf{0}$ & 0 & 1 & $\mathbf{a}$ & 0 & 0 & 1 & $\mathbf{a}$ \\
\hline Northen Totals & 3,512 & 33,232 & 2,616 & 32,714 & 554 & 5,776 & 243 & 4,851 & 177 & 8,133 & 7,102 & 84,706 \\
\hline \multicolumn{13}{|l|}{ Commodity-Carrying } \\
\hline -Short Haul & 1,083 & 12,748 & 403 & 7,063 & 777 & 6,360 & 330 & 6,646 & 174 & 7,164 & 2,767 & 39,981 \\
\hline -Long Haul & 62 & 3,208 & 54 & 921 & 27 & 268 & 54 & 3,516 & 214 & 20,302 & 412 & 28,215 \\
\hline \multicolumn{13}{|l|}{ Non-Commodity-Carrying } \\
\hline -Business Use & 1,283 & 14,868 & 580 & 8,308 & 202 & 1,290 & 42 & 620 & 9 & 415 & 2,116 & 25,501 \\
\hline -Penonal Use & 5,208 & 58,683 & 2,902 & 28,466 & 143 & 667 & 18 & 39 & 1 & 3 & 8,274 & 87,858 \\
\hline -Not in Use & 0 & $\mathbf{0}$ & $\mathbf{0}$ & 0 & 13 & 53 & 0 & $\mathbf{0}$ & 2 & 17 & 15 & 71 \\
\hline Midmed Totel & 7,636 & 89,507 & 3,940 & 44,758 & 1,162 & 8,639 & 444 & 10,821 & 401 & 27,901 & 13,583 & 181,626 \\
\hline \multicolumn{13}{|l|}{ Commodity-Carrying } \\
\hline -Short Haul & 1,446 & 14,537 & 480 & 6,628 & 740 & 11,243 & 264 & 6,395 & 257 & 9,354 & 3,187 & 48,158 \\
\hline -Long Haul & 66 & 357 & 76 & 606 & 30 & 629 & 44 & 2,260 & 180 & 17,400 & $3 \operatorname{sis}$ & 21,253 \\
\hline \multicolumn{13}{|l|}{ Non-Commodity-Carrying } \\
\hline -Buriness Use & 1,655 & 23,009 & 917 & 8,087 & 325 & 2,223 & 38 & 586 & 32 & 745 & 2957 & 34,650 \\
\hline -Personal Use & 9,046 & 89,526 & 3,943 & 49,370 & 166 & 753 & 41 & 178 & 10 & 69 & 13,05 & 139897 \\
\hline -Not in Use & 31 & 25 & 3 & $\mathbf{2 3}$ & 23 & 106 & 5 & 21 & 3 & 24 & $c s$ & 200 \\
\hline Sounth Totels & 12,244 & 127,455 & 5,420 & 64,715 & 1,283 & 14,955 & 393 & 9,441 & 481 & 27,592 & 19,801 & 244,157 \\
\hline Commodity-Carrying & & & & & & & & & & & & \\
\hline -Short Haul & 826 & 10,848 & 338 & 6,880 & 429 & 6,867 & 125 & 2,344 & 146 & 5,332 & 1865 & 32,271 \\
\hline -Long Haul & 42 & 929 & 3 & 9 & 20 & 110 & 19 & 613 & 86 & 8,030 & $1 \%$ & 9,692 \\
\hline \multicolumn{13}{|l|}{ Non-Commodity-Carrying } \\
\hline -Butinces Use & 1,258 & 13,443 & 512 & 4,735 & 114 & 1,677 & 15 & 122 & 17 & 275 & 1,915 & 20,251 \\
\hline -Perconal Use & 6,349 & 48,225 & 3,152 & 30,316 & 79 & 568 & 4 & 5 & 1 & 7 & 9,584 & 79,121 \\
\hline$-\mathrm{Not}$ in Uec & 27 & 226 & 10 & 38 & 34 & 51 & 0 & $\mathbf{0}$ & 1 & 8 & $n$ & 303 \\
\hline Wen Tots & 8,503 & 73,671 & 4,014 & 41,977 & 675 & 9,274 & 163 & 3,085 & 251 & 13,651 & 13,605 & 141,658 \\
\hline \multicolumn{13}{|l|}{ Commodity-Carrying } \\
\hline -Short Haul & 3,766 & 43,692 & 1,593 & 25,545 & 2,306 & 29,037 & 919 & 19,165 & 682 & 25,194 & 9,265 & 142,633 \\
\hline -Long Haul & 175 & 4,521 & 157 & 2,579 & 88 & 1,153 & 132 & 7,115 & 545 & 50,391 & $1,0 \% 7$ & 65,760 \\
\hline \multicolumn{13}{|l|}{ Non-Commodity-Carrying } \\
\hline -Burinces Use & 4,770 & 59,309 & 2,560 & 30,473 & 779 & 6,066 & 123 & 1,674 & 66 & 1,563 & 8297 & 99,035 \\
\hline -Personal Use & 23,125 & 216,091 & 11,668 & 125,506 & 433 & 2,177 & 63 & 223 & 12 & 79 & 35,301 & 344,075 \\
\hline -Not in Use & 59 & 252 & 12 & 61 & 69 & 210 & 6 & 22 & 6 & 50 & 152 & 59 \\
\hline UNITED STATES TOTALS & 31,ges & 323865 & 15,990 & 184,164 & 3,674 & 39,64 & 1,242 & 28,198 & 1,311 & $\pi, 2 \pi$ & 54,112 & 652,147 \\
\hline
\end{tabular}

(a) Quantity rounded to zero. 


\section{APPENDIX A \\ DESIGN OF THE \\ 1990 NATIONWIDE TRUCK ACTIVITY AND COMMODITY SURVEY}

\section{Purpose of Survey}

The NTACS was designed by the Census Bureau with DOT consultation and involvement to provide detailed information on daily activity patterns of trucks and on the relationships of trucking to commodity movements and economic activity. The NTACS measures the 1990 activity of trucks registered on July 1, 1987, and in the scope of the Census Bureau's 1987 Truck Inventory and Use Survey (TIUS).

\section{Universe}

The universe for the 1990 NTACS includes the following: All trucks during the NTACS period (October 29, 1989--October 27, 1990) registered in one of the 50 states or the District of Columbia on July 1, 1987, and operating in 1987 as estimated by the 1987 TIUS.

A. A "truck" is a vehicle registered in a state as a truck, or a vehicle with a "truck" chassis registered as a car. Vehicles excluded from the 1987 TIUS and subsequently excluded from the 1990 NTACS universe were:

1. Vehicles owned by Federal, state, or local governments

2. Ambulances

3. Buses

4. Mobile homes

5. Trucks

- Sold prior to 1987 (NTACS only)

- Disposed of prior to July 1, 1986 (TIUS and NTACS)

6. Farm tractors

7. Unpowered trailers

8. Trucks reported scrapped or wrecked prior to registration year. 


\section{Survey Frame}

The 1990 NTACS sample was selected from commodity-carrying trucks and noncommodity carrying business, personal transportation and idle trucks which responded to the 1987 TIUS. Thus the NTACS sdmple is a subsample of the 1987 TIUS sample respondents.

The 1987 TIUS frame is described below.

\section{A. Description}

The 1987 TIUS was based on a sample survey of approximately 135,321 trucks (Table B) selected from the TIUS universe of approximately 45,393,671 trucks (Table A). R.L. Polk and Company, as directed by the Census Bureau, selected a stratified random sample from vehicle registration files for each of the 50 states and the District of Columbia. Five vehicle type strata were selected:

\begin{tabular}{|c|l|}
\hline STRATUM & \multicolumn{1}{|c|}{ VEHICLE TYPE } \\
\hline 1 & Pickup trucks \\
2 & Vans, panels and utilities \\
3 & Single-unit, small $\left(\mathrm{GVW}^{1} \leq 26,000 \mathrm{lbs}\right.$. $)$ \\
4 & Single-unit, large $(\mathrm{GVW}>26,000 \mathrm{lbs})$. \\
5 & Truck-tractors \\
\hline
\end{tabular}

The truck (unit) response rate for the 1987 TIUS at the U.S. level was approximately 77.9 percent, i.e. appr oximately 104,601 trucks responded (Table C).

The 1987 TIUS forms were mailed to registered owners of each sample truck to determine the 1987 operational and physical characteristics. Based on the 1987 TIUS, the NTACS frame contained the following information:

1. State of registration

2. Vehicle type (stratum)

3. 1987 TIUS expansion factor

1 Gross Vehicle Weight rating (GVW) is the weight of a vehicle when loaded to its capacity.

$$
\text { A }-2
$$




\section{Response characteristics}

5. 1987 TIUS characteristics.

Trucks identified as idled (i.e., wrecked, awaiting repair, etc.) from the 1987 TIUS were given a chance for selection in the NTACS because these trucks could have been operational at the time the NTACS survey was conducted. Trucks scrapped or not in use in 1987 were excluded from the survey. No attempt was made to follow up "deaths" or to include newly registered trucks that were registered since July 1,1987 . If a vehicle changed ownership, however, an attempt was made to find the new owner of the truck.

\section{B. Preparation of the Frame}

The 103,737 trucks (Table D) in the sampling frame were stratified into one of 225 strata defined by geographic division, types of haul, and truck classification. Type of haul was either long or short for commodity-carrying trucks, or business use only, personal use only, or idled non-commodity-carrying trucks. A longhaul commodity-carrying truck is a truck with 30 percent of its annual mileage on trips of 200 miles or more; or at least 50 percent of its annual mileage on trips of 50 to 200 miles and at least 10 percent of annual mileage on trips of 200 miles or more.

Prior to sample selection, trucks within each stratum were sorted by body type and ZIP.

\section{Sample Size}

Using the 1982 TIUS as a basis of study and later adjusted by results from the 1987 TIUS, the NTACS sample was determined to be approximately 44,002 trucks (Table E). Of those, approximately 14,000 were long-haul commodity-carrying, approximately 24,000 were short-haul commodity-carrying, and the balance were non-commodity-carrying. Sample sizes were determined to provide reliable estimates of annual miles at the division level for commodity-carrying large trucks plus tractor-trailer trucks, commodity-carrying pick-ups plus vans, all commoditycarrying trucks, all large plus tractor-trailer trucks, all pick-ups plus vans, and all trucks.

Approximately 42 percent of all trucks responding in the 1987 TIUS were included in the 1990 NTACS sample.

The estimated 1990 NTACS universe size (using XPFD1) is approximately 41,768,000 (see Table 1.1). 


\section{Sample Selection}

The survey collected information on the activities of trucks on one or two sample days. The sample was selected in two steps: (1) a sample of trucks was identified from the in-scope list of trucks in the 1987 TIUS, and (2) one or two sample days for each specific truck was selected.

A respondent was asked to provide data on a specific day within a specific week(s). In addition, within the specific week(s), the truck reported which day(s) it operated. For the selected day(s), the owner of a truck reported detailed information on its characteristics and activities for a day and/or for the trips started and continuing on the sample day.

\section{A. Selection of Trucks}

A stratified systematic random sample of trucks from the 1987 TIUS was included in the 1990 NTACS.

Selected into the 1990 NTACS were the following groups of trucks responding to the 1987 TIUS:

- All commodity-carrying long-haul trucks

- All commodity-carrying short-haul pickups and vans

- A subsample of commodity-carrying short-haul tractor-trailers; single-unit, large; and single-unit small trucks

- A subsample of non-commodity-carrying trucks.

ORNL's current understanding of the number of NTACS respondents $(22,044)$ and NTACS nonrespondents $(21,808)$ is given in Table G. It should be noted that 150 trucks $(=44,002-22,044-21,808)$ in the original NTACS sample were eventually determined to be out of scope for NTACS.

\section{B. Selection of Weeks}

Each long-haul commodity-carrying truck was enumerated for two selected oneweek periods. The year was divided into 13 blocks (periods) of four-weeks each beginning October 29, 1989, and ending October 27, 1990. Each truck was randomly assigned to a four-week block (period).

The first week was randomly selected from the first two-weeks in the block; the second week was two-weeks later. The sample of trucks for a block was evenly divided between the first and second week. For each day in the two-weeks 
selected, the owner of the truck was asked to report whether the truck operated some time during the day.

Each selected local haul commodity-carrying and non-commodity-carrying truck was enumerated for one selected one-week period during the four-week block.

\section{Selection of Days}

For each selected week, each truck was randomly assigned a day to report daily characteristics. One substitute day was provided on the questionnaire. If commodities were carried during the week, but not on either the selected or a substitute day, the respondent was provided a telephone number to call to obtain a new sample day within the sampled week. For long-haul trucks, each day of the week had an equal probability of selection.

\section{Definition of Trip}

A trip is defined as travel starting when a truck left the base or location of a pickup or delivery and ended when it arrived at the base or location of the next pickup or delivery (this includes traveling empty).

\section{E. Selection of Trip}

For long-haul trucks, a trip in which any part of the travel occurred on the sample day was selected into the survey. This allowed information on any trip beginning before the sample day, continuing into the sample day, and ending after the sample day to be collected.

\section{NTACS Questionnaire Content}

See Appendix C.

\section{How Conducted}

\section{A. General}

The 1990 NTACS was conducted by mail. The registration information was computer imprinted on the questionnaires identifying the specific vehicle selected as well as the week(s) and day(s) selected. 


\section{B. Mailout}

Selected sampled cases were assigned to one of 13 periods. Each respondent received one of two questionnaires requesting the activities of the selected vehicle for a selected day in a four-week period of the year or activities for two selected days in different weeks of the same four-week period.

The mailing package consisted of the original questionnaire, instruction sheet, cover letter, and return envelope.

\section{Receipt and Check-in}

Questionnaires were mailed from the Census Bureau's Jeffersonville, Indiana facility. Respondents were requested to return these report forms to that facility immediately after the sample day where an automated check-in occurred. Respondents with delinquent reports were followed up.

\section{Follow-up}

In an attempt to improve the response rate for the survey, two mail follow-ups were conducted. In addition, a telephone follow-up was initially conducted for the first five (5) periods. Due to the high cost in conducting telephone followup to obtain a completed response, the use of certified mail was implemented for the remaining eight (8) periods. Certified mail proved to be cost effective and improved not only overall response, but item non-response as well.

\section{E. Data Editing}

Responses were edited for reasonableness and consistency through clerical screening and a computer edit. The physical characteristics of the selected vehicles were compared with those attributes reported in the 1987 TIUS. The operational characteristics of the sampled vehicles were edited against parameters developed from industry standards and from knowledge of the operations of various carrier types.

It was recognized during the design and development stages and from the results of the pretest that this data collection would be an exploratory process which would attempt to study new and changing conditions in truck operation and activity characteristics that had not been measured in any previous program.

During the edit process, a number of important observations were made that will help improve response and data quality for the 1994 program. In preparation for the 1994 program, additional contacts with trade groups, TIUS participants, and DOT specialists will improve questionnaire design and respondent reportability. The evaluation of the 1990 data collection will 
improve data item definitions and instructions and therefore improve response rates. These steps will be part of a process to balance data user needs and respondent ability to report quality data on a timely basis. 
TABLE A 1987 TIUS NUMBER OF TRUCKS IN UNIVERSE BY STATE BY TRUCK TYPE (9/2/88)

\begin{tabular}{|c|c|c|c|c|c|}
\hline \multirow[b]{2}{*}{ BTATE } & \multicolumn{5}{|c|}{ TRUCK TYPE } \\
\hline & $\begin{array}{c}1 \\
\text { PU }\end{array}$ & $\begin{array}{c}2 \\
\text { VAN }\end{array}$ & LT TAK & HWY TRK & $\stackrel{5}{5}$ TRACTOR \\
\hline$A L$ & 638,613 & 173,339 & 51,492 & 26,700 & 24,042 \\
\hline AK & 107,061 & 52,685 & 7,485 & 3,419 & 2,172 \\
\hline$A Z$ & 492,522 & 206,317 & 49,082 & 13,493 & 10,295 \\
\hline$\overline{A R}$ & 427,842 & 96,058 & 3,097 & 930 & 6,002 \\
\hline CA & $3,114,535$ & $1,295,855$ & 262,097 & 52,980 & 112,338 \\
\hline $\mathrm{CO}$ & 591,810 & 309,819 & 57,336 & 21,496 & 4,602 \\
\hline CT & 237,758 & 161,962 & 35,245 & 12,614 & 7,141 \\
\hline DE & 63,762 & 38,851 & 10,400 & 5,511 & 3,604 \\
\hline$D C$ & 6,868 & 12,387 & 1,980 & 589 & 93 \\
\hline FL & $1,079,807$ & 607,560 & 97,195 & 27,182 & 45,273 \\
\hline QA & 808,816 & 280,588 & 86,280 & 27,734 & 32,606 \\
\hline $\mathrm{HI}$ & 109,845 & 40,725 & 10,726 & 3,538 & 1,612 \\
\hline ID & 225,104 & 68,335 & 20,055 & 6,038 & 5,830 \\
\hline II. & 775,257 & 547,659 & 137,478 & 73,488 & 98,863 \\
\hline $\mathbb{N}$ & 623,625 & 288,552 & 97,405 & 39,010 & 43,743 \\
\hline IA & 414,034 & 147,030 & 65,749 & 42,663 & 18,678 \\
\hline KS & 468,723 & 148,444 & 82,097 & 30,477 & 15,028 \\
\hline$K Y$ & 552,570 & 167,666 & 70,020 & 19,028 & 15,610 \\
\hline LA & 662,206 & 203,953 & 52,191 & 19,955 & 16,887 \\
\hline ME & 170,586 & 61,024 & 17,425 & 10,595 & 6,144 \\
\hline$M D$ & 341,390 & 227,197 & 59,194 & 31,332 & 14,563 \\
\hline$M A$ & 315,869 & 287,985 & 58,170 & 38,502 & 11,600 \\
\hline MI & 862,631 & 568,562 & 78,971 & 21,405 & 44,070 \\
\hline MN & 514,359 & 235,517 & 61,950 & 37,574 & 16,886 \\
\hline MS & 399,959 & 83,261 & 18,734 & 7,364 & 8,762 \\
\hline MO & 718,100 & 184,820 & 78,035 & 24,290 & 24,702 \\
\hline MT & 221,400 & 63,818 & 22,815 & 6,283 & 8,008 \\
\hline NE & 272,798 & 79,040 & 62,850 & 19,971 & 10,830 \\
\hline NV & 171,537 & 65,615 & 9,500 & 1,955 & 3,546 \\
\hline NH & 137,873 & 71,246 & 17,462 & 7,337 & 5,586 \\
\hline$N$ & 330,317 & 349,862 & 77,012 & 41,693 & 20,226 \\
\hline NM & 333,041 & 107,392 & 23,401 & 4,053 & 5,301 \\
\hline NY & 693,191 & 651,353 & 137,931 & 49,282 & 33,201 \\
\hline NC & 858,052 & 311,547 & 126,308 & 36,738 & 43,861 \\
\hline ND & 147,705 & 42,202 & 67,000 & 10,383 & 0,153 \\
\hline $\mathrm{OH}$ & 927,108 & 581,007 & 134,423 & 87,165 & 35,001 \\
\hline OK & 644,308 & $167,4 \pi 7$ & 67,030 & 18,305 & 24.770 \\
\hline OA & 571,409 & 208,582 & 34,726 & 9,987 & 22,746 \\
\hline PA & 869,677 & 611,082 & 121,812 & 55,690 & 52,873 \\
\hline AI & 61,736 & 43,657 & 10,594 & 3,451 & 2,740 \\
\hline SC & 407,583 & 132,578 & 44,075 & 12,206 & 0,020 \\
\hline SD & 158,308 & 46,808 & 25,240 & 12,141 & 6,460 \\
\hline$T N$ & 708,475 & 217,247 & 57,914 & 20,551 & 20,773 \\
\hline$T x$ & $2,703,736$ & 997,144 & 196,460 & 44,588 & $98, \infty 9$ \\
\hline UT & 240,582 & 112,077 & 20,544 & 5,601 & 7,076 \\
\hline$v r$ & 79,227 & 38,120 & 0,187 & 4,500 & 1,007 \\
\hline VA & 696,407 & 310,065 & 84,383 & 30,308 & 23,336 \\
\hline WA & 739,707 & 289,801 & 56,688 & 11,731 & 19,2223 \\
\hline$w$ & 270,632 & 110,650 & 33,218 & 9,512 & 4,136 \\
\hline WI & 480,254 & 202,198 & 68,387 & 40,078 & 23,769 \\
\hline$w r$ & 145,669 & 57,020 & 14,834 & 3,438 & 7,245 \\
\hline US & $27,595,406$ & $12,444,370$ & $3,093,709$ & $1,145,062$ & $1,115,124$ \\
\hline & & & & GRAND & $\perp \quad 46,3 \times 3,07$ \\
\hline
\end{tabular}

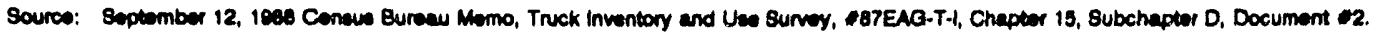


TABLE B. 1987 TIUS PRELIMINARY SAMPLE SIZES BY STATE BY TRUCK TYPE

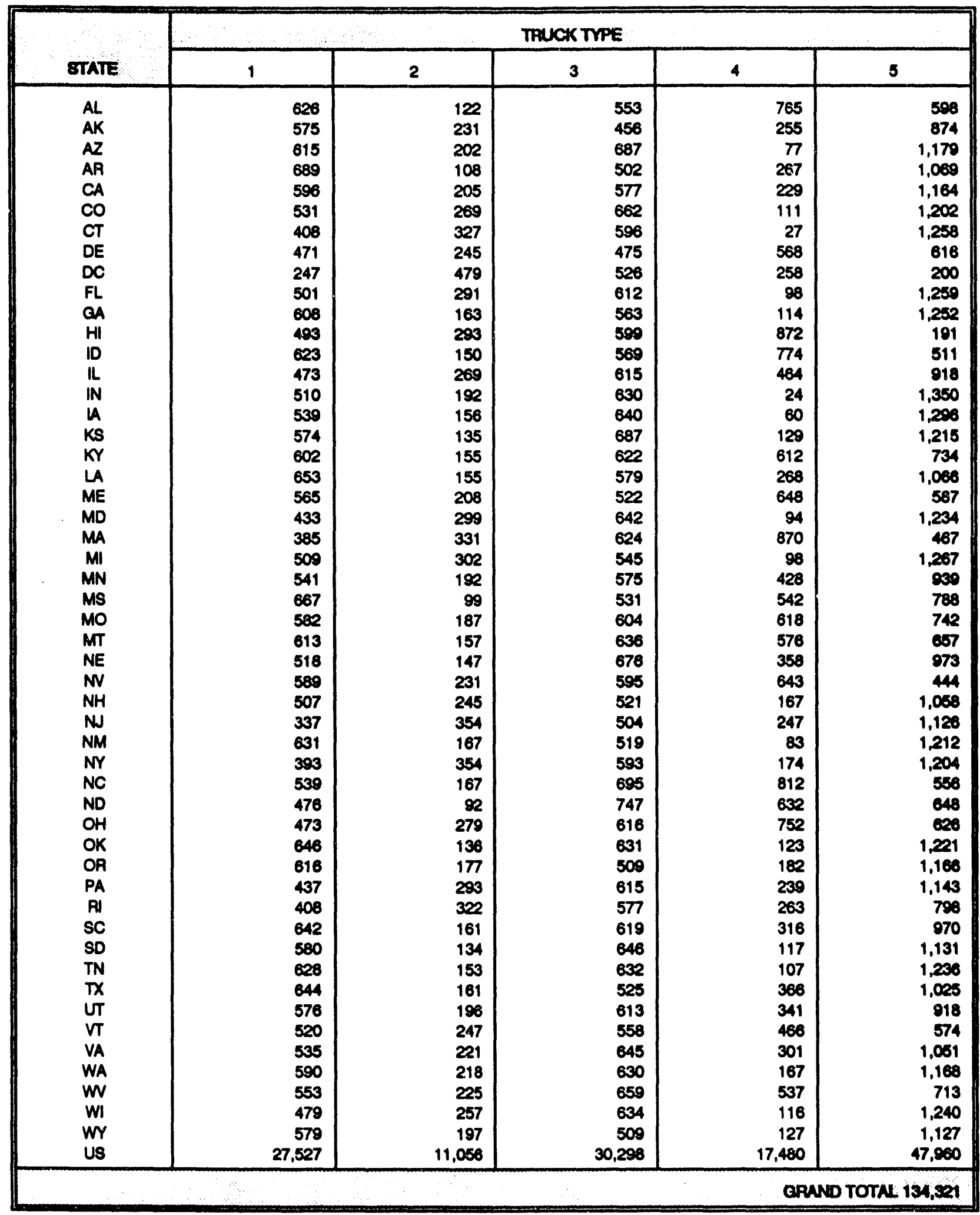

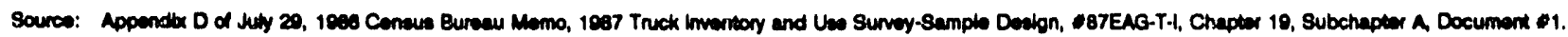


TABLE C. 1987 TIUS NUMBER OF SAMPLE RESPONDENTS BY STATE BY TRUCK TYPE

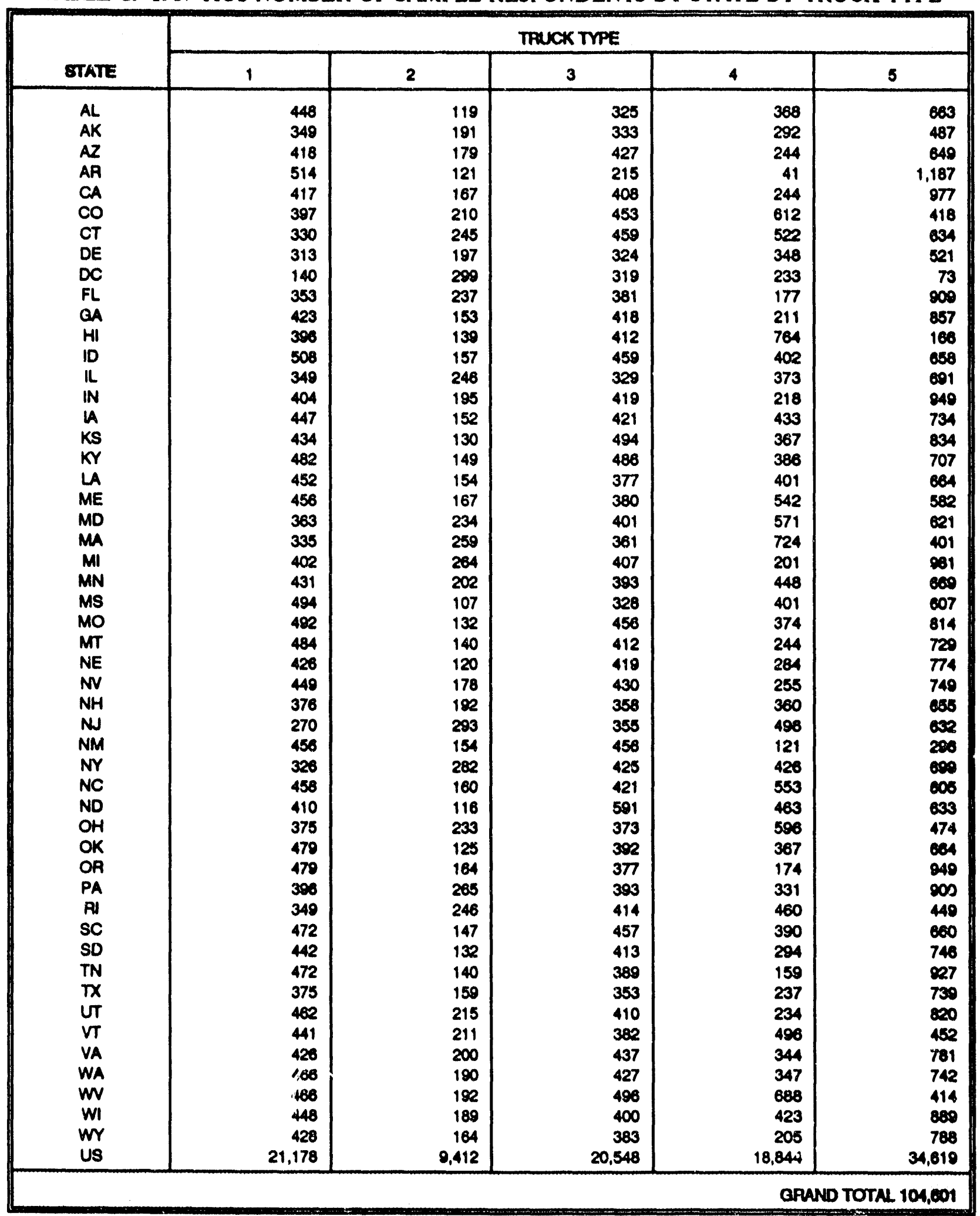

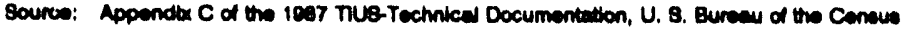


TABLE D. RESTRATIFICATION OF 1987 TIUS NUMBER OF SAMPLE RESPONDENTS BY DIVISION BY COMMODITY-CARRYING STATUS BY TRUCK TYPE FOR N1'ACS SAMPLING (FRMWT TRUCK COUNT)

\begin{tabular}{|c|c|c|c|c|c|c|c|}
\hline \multirow{2}{*}{$\begin{array}{l}\text { census } \\
\text { DMsions }\end{array}$} & \multirow{2}{*}{$\begin{array}{l}\text { Covinootir- } \\
\text { CAFTMNO STATUS }\end{array}$} & \multicolumn{6}{|c|}{ TPUCK TYPE } \\
\hline & & 1 & 2 & 3 & 4 & 5 & TOTAL \\
\hline $\begin{array}{l}1 \text { Now } \\
\text { England } \\
\text { (ME VT R } \\
\text { NH MA CT) }\end{array}$ & $\begin{array}{l}1 \\
2 \\
3 \\
4 \\
5\end{array}$ & $\begin{array}{r}302 \\
11 \\
361 \\
1,560 \\
7\end{array}$ & $\begin{array}{r}173 \\
11 \\
231 \\
872 \\
5\end{array}$ & $\begin{array}{r}1,452 \\
54 \\
488 \\
274 \\
39\end{array}$ & $\begin{array}{r}2,389 \\
232 \\
308 \\
98 \\
36\end{array}$ & $\begin{array}{r}2,036 \\
855 \\
164 \\
40 \\
27\end{array}$ & 12,025 \\
\hline $\begin{array}{l}2 \text { Middlo } \\
\text { Atlantic } \\
\text { (NY NW PA) }\end{array}$ & $\begin{array}{l}1 \\
2 \\
3 \\
4 \\
5\end{array}$ & $\begin{array}{r}114 \\
2 \\
149 \\
705 \\
0\end{array}$ & $\begin{array}{r}107 \\
7 \\
156 \\
550 \\
1\end{array}$ & $\begin{array}{r}741 \\
18 \\
282 \\
91 \\
20\end{array}$ & $\begin{array}{r}970 \\
61 \\
133 \\
61 \\
12\end{array}$ & $\begin{array}{r}1,322 \\
738 \\
98 \\
9 \\
23\end{array}$ & 6,370 \\
\hline $\begin{array}{l}3 \text { Eact North } \\
\text { Contral } \\
\text { (OH IN IL } \\
\text { MI WI) }\end{array}$ & $\begin{array}{l}1 \\
2 \\
3 \\
4 \\
5\end{array}$ & $\begin{array}{r}228 \\
16 \\
287 \\
1,407 \\
4\end{array}$ & $\begin{array}{r}129 \\
12 \\
141 \\
325 \\
2\end{array}$ & $\begin{array}{r}1,173 \\
43 \\
413 \\
252 \\
22\end{array}$ & $\begin{array}{r}1,107 \\
173 \\
175 \\
293 \\
26\end{array}$ & $\begin{array}{r}1,989 \\
1,726 \\
150 \\
23 \\
27\end{array}$ & 10,643 \\
\hline $\begin{array}{l}4 \text { Weat North } \\
\text { Contral } \\
\text { (MN A MO } \\
\text { ND SD NE } \\
\text { KS) }\end{array}$ & $\begin{array}{l}1 \\
2 \\
3 \\
4 \\
5\end{array}$ & $\begin{array}{r}513 \\
19 \\
584 \\
1,913 \\
14\end{array}$ & $\begin{array}{r}90 \\
12 \\
167 \\
704 \\
4\end{array}$ & $\begin{array}{r}2,303 \\
46 \\
435 \\
317 \\
55\end{array}$ & $\begin{array}{r}1,930 \\
266 \\
188 \\
195 \\
50\end{array}$ & $\begin{array}{r}2,592 \\
2,268 \\
199 \\
55 \\
37\end{array}$ & 14,854 \\
\hline $\begin{array}{l}5 \text { South } \\
\text { Attantic } \\
\text { (DE MD CC } \\
\text { VA WW NC } \\
\text { SC CA FL) }\end{array}$ & $\begin{array}{l}1 \\
2 \\
3 \\
4 \\
5\end{array}$ & $\begin{array}{r}355 \\
17 \\
449 \\
2,528 \\
14\end{array}$ & $\begin{array}{r}181 \\
17 \\
315 \\
1,273 \\
5\end{array}$ & $\begin{array}{r}2.12 \\
88 \\
822 \\
520 \\
48\end{array}$ & $\begin{array}{r}2,432 \\
250 \\
385 \\
348 \\
47\end{array}$ & $\begin{array}{r}3,165 \\
1,758 \\
272 \\
87 \\
47\end{array}$ & 17,515 \\
\hline $\begin{array}{l}6 \text { Eact South } \\
\text { Contral } \\
\text { (KY TN } \\
\text { NL MS) }\end{array}$ & $\begin{array}{l}1 \\
2 \\
3 \\
4 \\
5\end{array}$ & $\begin{array}{r}205 \\
7 \\
224 \\
1,430 \\
3\end{array}$ & $\begin{array}{r}54 \\
14 \\
68 \\
376 \\
2\end{array}$ & $\begin{array}{r}870 \\
27 \\
350 \\
221 \\
28\end{array}$ & $\begin{array}{r}823 \\
230 \\
108 \\
22 \\
20\end{array}$ & $\begin{array}{r}1,384 \\
1,152 \\
174 \\
88 \\
42\end{array}$ & 8,004 \\
\hline $\begin{array}{l}7 \text { Wot South } \\
\text { Contrel } \\
\text { (AR LA } \\
\text { OK DA }\end{array}$ & $\begin{array}{l}1 \\
2 \\
3 \\
4 \\
5\end{array}$ & $\begin{array}{r}182 \\
11 \\
312 \\
1.276 \\
14\end{array}$ & $\begin{array}{r}30 \\
7 \\
97 \\
408 \\
2 \\
\end{array}$ & $\begin{array}{r}811 \\
36 \\
308 \\
128 \\
39\end{array}$ & $\begin{array}{r}733 \\
96 \\
143 \\
25 \\
32\end{array}$ & $\begin{array}{r}1,723 \\
1,145 \\
231 \\
23 \\
55\end{array}$ & 7,872 \\
\hline $\begin{array}{l}8 \text { Mountain } \\
\text { (MT ID WY } \\
\text { CO NM AZ } \\
\text { UT NM }\end{array}$ & $\begin{array}{l}1 \\
2 \\
3 \\
4 \\
5\end{array}$ & $\begin{array}{r}383 \\
34 \\
558 \\
2,569 \\
18\end{array}$ & $\begin{array}{r}107 \\
5 \\
174 \\
1,091 \\
5\end{array}$ & $\begin{array}{r}1,953 \\
107 \\
732 \\
515 \\
106 \\
\end{array}$ & $\begin{array}{r}1,636 \\
434 \\
252 \\
88 \\
48 \\
\end{array}$ & $\begin{array}{r}2,973 \\
2,168 \\
335 \\
64 \\
97\end{array}$ & 16,452 \\
\hline $\begin{array}{l}\text { O Pecific } \\
\text { WA OA CA } \\
\text { AK HI }\end{array}$ & $\begin{array}{l}1 \\
2 \\
3 \\
4 \\
5\end{array}$ & $\begin{array}{r}201 \\
8 \\
281 \\
1,588 \\
6\end{array}$ & $\begin{array}{r}90 \\
8 \\
131 \\
608 \\
6\end{array}$ & $\begin{array}{r}1,168 \\
25 \\
381 \\
305 \\
31\end{array}$ & $\begin{array}{r}1,540 \\
54 \\
159 \\
16 \\
18\end{array}$ & $\begin{array}{r}2,284 \\
755 \\
109 \\
23 \\
20\end{array}$ & 9,002 \\
\hline
\end{tabular}

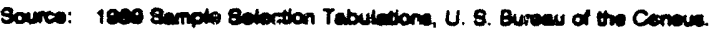
Des: $10 / 13 / 00$ 
TABLE E NUMBER OF TRUCKS SELECTED FOR 1989 NTACS SAMPLE BY

DIVISION BY COMMODITY-CARRYING BY TRUCK TYPE (TRUCK COUNT)

\begin{tabular}{|c|c|c|c|c|c|c|c|}
\hline \multirow{2}{*}{$\begin{array}{l}\text { CENSUS } \\
\text { DMSENN }\end{array}$} & \multirow{2}{*}{$\begin{array}{l}\text { coninoorty } \\
\text { chraniv } \\
\text { staTus }\end{array}$} & \multicolumn{6}{|c|}{ TAUCK TYPE } \\
\hline & & 1 & 2 & 3 & 4 & 5 & TOTAL \\
\hline 1 & $\begin{array}{l}1 \\
2 \\
3 \\
4 \\
5\end{array}$ & $\begin{array}{r}283 \\
11 \\
36 \\
120 \\
4\end{array}$ & $\begin{array}{r}168 \\
11 \\
60 \\
60 \\
5\end{array}$ & $\begin{array}{r}640 \\
54 \\
40 \\
40 \\
18\end{array}$ & $\begin{array}{r}922 \\
219 \\
20 \\
5 \\
15\end{array}$ & $\begin{array}{r}717 \\
814 \\
11 \\
5 \\
13 \\
\end{array}$ & 4,201 \\
\hline 2 & $\begin{array}{l}1 \\
2 \\
3 \\
4 \\
5\end{array}$ & $\begin{array}{r}114 \\
2 \\
8 \\
84 \\
0\end{array}$ & $\begin{array}{r}102 \\
7 \\
80 \\
84 \\
1\end{array}$ & $\begin{array}{r}712 \\
18 \\
121 \\
61 \\
10\end{array}$ & $\begin{array}{r}951 \\
60 \\
38 \\
2 \\
5\end{array}$ & $\begin{array}{r}600 \\
710 \\
23 \\
1 \\
9\end{array}$ & 3,804 \\
\hline 3 & $\begin{array}{l}1 \\
2 \\
3 \\
4 \\
5\end{array}$ & $\begin{array}{r}217 \\
16 \\
50 \\
75 \\
2\end{array}$ & $\begin{array}{r}128 \\
12 \\
50 \\
75 \\
2\end{array}$ & $\begin{array}{r}762 \\
41 \\
50 \\
75 \\
11\end{array}$ & $\begin{array}{r}355 \\
164 \\
40 \\
1 \\
13\end{array}$ & $\begin{array}{r}452 \\
1,598 \\
25 \\
2 \\
12\end{array}$ & 4,228 \\
\hline 4 & $\begin{array}{l}1 \\
2 \\
3 \\
4 \\
5\end{array}$ & $\begin{array}{r}503 \\
19 \\
80 \\
150 \\
7\end{array}$ & $\begin{array}{r}90 \\
12 \\
80 \\
150 \\
4\end{array}$ & $\begin{array}{r}1,200 \\
46 \\
75 \\
100 \\
27\end{array}$ & $\begin{array}{r}1,556 \\
241 \\
35 \\
35 \\
23\end{array}$ & $\begin{array}{r}397 \\
2,097 \\
12 \\
15 \\
17\end{array}$ & 6,971 \\
\hline 5 & $\begin{array}{l}1 \\
2 \\
3 \\
4 \\
5\end{array}$ & $\begin{array}{r}341 \\
17 \\
80 \\
150 \\
7\end{array}$ & $\begin{array}{r}175 \\
17 \\
80 \\
150 \\
3\end{array}$ & $\begin{array}{r}741 \\
68 \\
121 \\
83 \\
24\end{array}$ & $\begin{array}{r}564 \\
240 \\
50 \\
75 \\
22\end{array}$ & $\begin{array}{r}750 \\
1,674 \\
50 \\
50 \\
22\end{array}$ & 5,562 \\
\hline 6 & $\begin{array}{l}1 \\
2 \\
3 \\
4 \\
5\end{array}$ & $\begin{array}{r}199 \\
7 \\
80 \\
150 \\
3\end{array}$ & $\begin{array}{r}53 \\
14 \\
62 \\
150 \\
1\end{array}$ & $\begin{array}{r}813 \\
25 \\
50 \\
50 \\
13\end{array}$ & $\begin{array}{r}286 \\
220 \\
9 \\
4 \\
10\end{array}$ & $\begin{array}{r}448 \\
1,067 \\
16 \\
3 \\
10\end{array}$ & 3,752 \\
\hline 7 & $\begin{array}{l}1 \\
2 \\
3 \\
4 \\
5\end{array}$ & $\begin{array}{r}177 \\
11 \\
50 \\
160 \\
7\end{array}$ & $\begin{array}{r}30 \\
7 \\
26 \\
70 \\
1\end{array}$ & $\begin{array}{r}772 \\
34 \\
52 \\
50 \\
19\end{array}$ & $\begin{array}{r}384 \\
92 \\
50 \\
25 \\
15\end{array}$ & $\begin{array}{r}912 \\
1,102 \\
79 \\
23 \\
27\end{array}$ & 4,184 \\
\hline 8 & $\begin{array}{l}1 \\
2 \\
3 \\
4 \\
5\end{array}$ & $\begin{array}{r}375 \\
34 \\
80 \\
150 \\
8\end{array}$ & $\begin{array}{r}105 \\
5 \\
80 \\
150 \\
5\end{array}$ & $\begin{array}{r}1,311 \\
103 \\
65 \\
80 \\
30\end{array}$ & $\begin{array}{r}538 \\
392 \\
84 \\
10 \\
24\end{array}$ & $\begin{array}{r}872 \\
1,938 \\
70 \\
10 \\
30\end{array}$ & 6,560 \\
\hline 9 & $\begin{array}{l}1 \\
2 \\
3 \\
4 \\
5\end{array}$ & $\begin{array}{r}200 \\
8 \\
80 \\
150 \\
2\end{array}$ & $\begin{array}{r}84 \\
8 \\
80 \\
150 \\
5\end{array}$ & $\begin{array}{r}1,124 \\
24 \\
50 \\
50 \\
13\end{array}$ & $\begin{array}{r}500 \\
54 \\
50 \\
16 \\
8\end{array}$ & $\begin{array}{r}1,100 \\
728 \\
50 \\
23 \\
12\end{array}$ & 4,576 \\
\hline
\end{tabular}

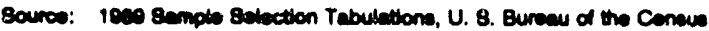

Den: $10 / 13 / 0$ 
TABLE F. ESTIMATED 1987 TIUS UNIVERSE SIZE' BASED ON 1987 TIUS SAMPLE (Compare with Table A.)

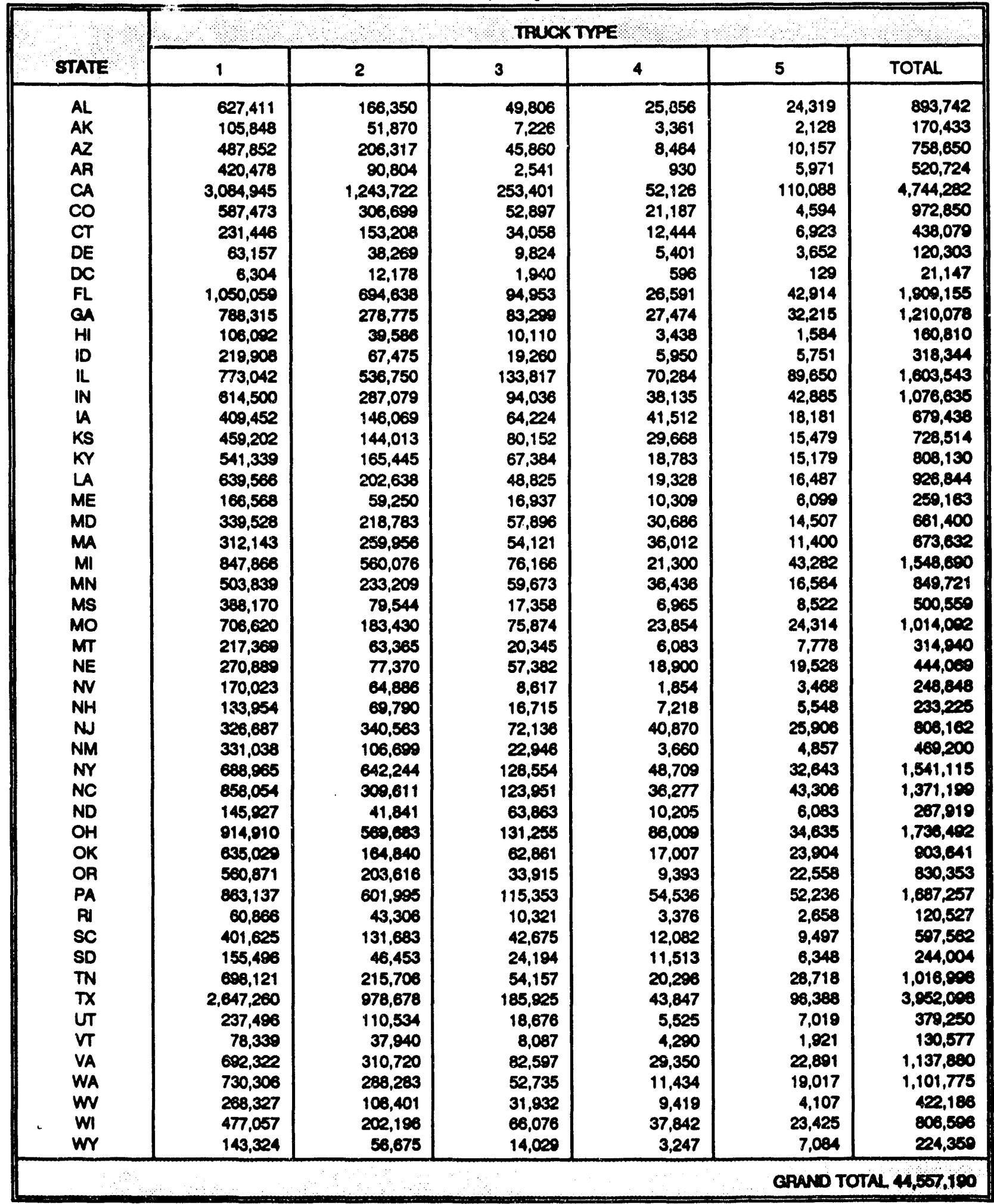

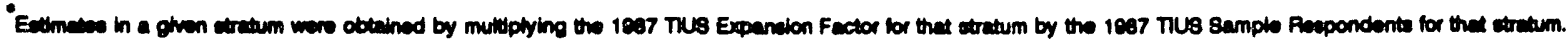

Sources: Appendices B and C of the 1987 ThLS - Technical Documentution

U. 8. Bureas of the Cenaus 
TABLE O. NUMBER OF SAMPLE RESPONDENTS' (NONRESPONDENTS) TO THE 1990 NTACS

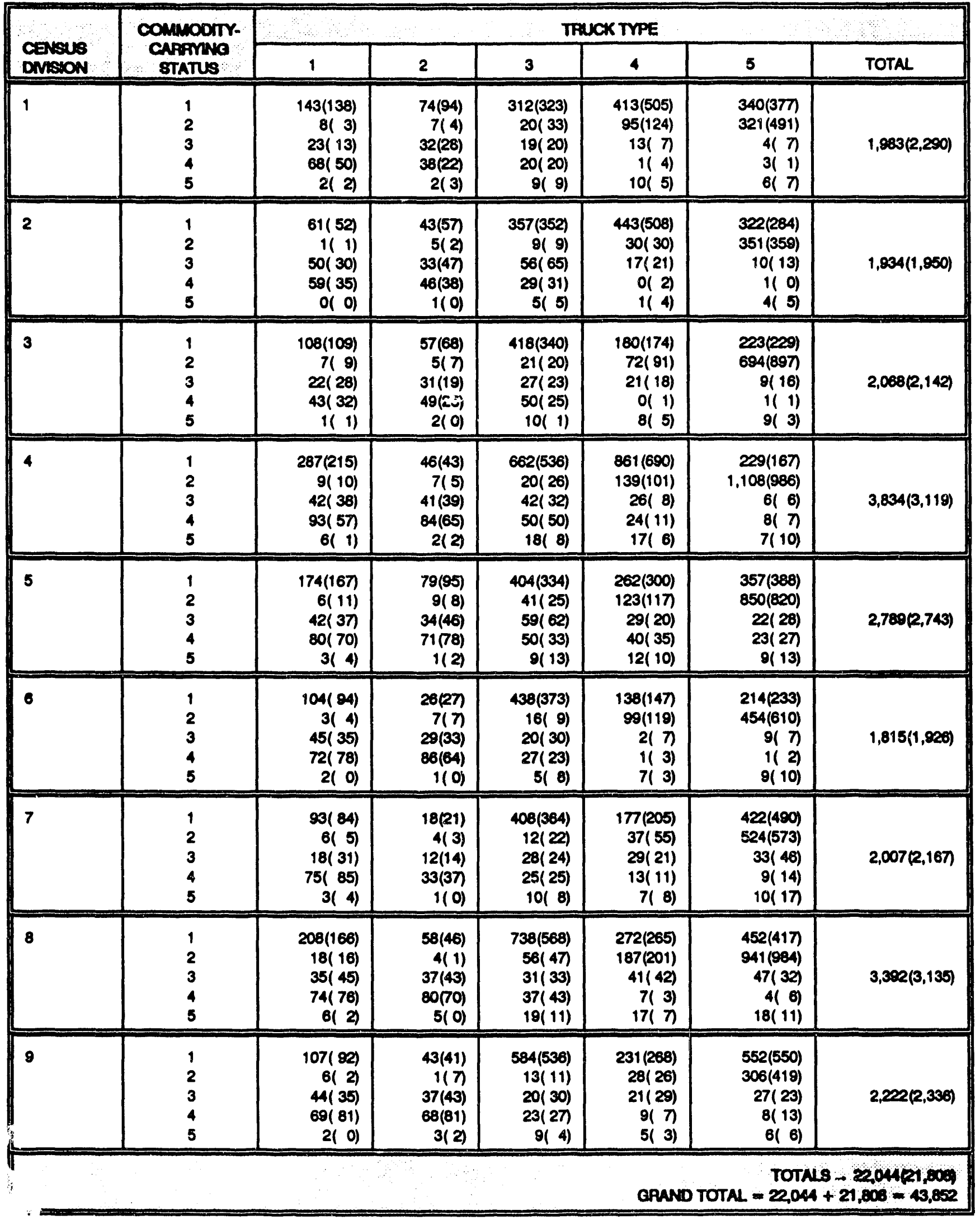

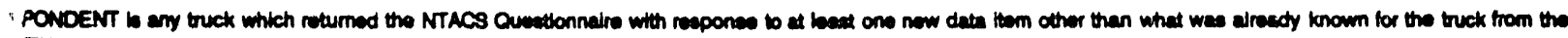
The. The

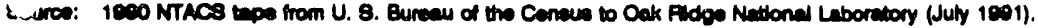


TABLE H. NUMBER OF SAMPLE DAY ONE RESPONDENTS (NONRESPONDENTS)

TO THE 1990 NTACS

\begin{tabular}{|c|c|c|c|c|c|c|c|}
\hline \multirow{2}{*}{$\begin{array}{l}\text { Censes } \\
\text { DNBSON }\end{array}$} & \multirow{2}{*}{ 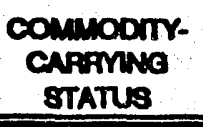 } & \multicolumn{6}{|c|}{ TFUCK TYPE } \\
\hline & & 1 & 2 & 3 & 4 & 5 & TOTAL \\
\hline 1 & $\begin{array}{l}1 \\
2 \\
3 \\
4 \\
5\end{array}$ & $\begin{array}{r}75(206) \\
4(7) \\
10(26) \\
37(81) \\
0(4)\end{array}$ & $\begin{array}{r}35(133) \\
3(8) \\
19(41) \\
22(38) \\
0(5)\end{array}$ & $\begin{array}{r}124(511) \\
5(48) \\
6(33) \\
6(34) \\
0(18)\end{array}$ & $\begin{array}{r}203(715) \\
46(173) \\
5(15) \\
0(5) \\
1(14)\end{array}$ & $\begin{array}{r}169(548) \\
151(661) \\
3(8) \\
1(3) \\
0(13)\end{array}$ & $925(3,348)$ \\
\hline 2 & $\begin{array}{l}1 \\
2 \\
3 \\
4 \\
5\end{array}$ & $\begin{array}{r}32(81) \\
0(2) \\
28(52) \\
33(61) \\
0(0)\end{array}$ & $\begin{array}{r}18(82) \\
4(3) \\
21(59) \\
28(56) \\
0(1)\end{array}$ & $\begin{array}{r}162(547) \\
1(17) \\
28(93) \\
9(51) \\
0(10)\end{array}$ & $\begin{array}{r}225(726) \\
18(42) \\
10(28) \\
0(2) \\
0(5)\end{array}$ & $\begin{array}{r}155(451) \\
157(553) \\
3(20) \\
0(1) \\
0(9)\end{array}$ & $832(2,052)$ \\
\hline 3 & $\begin{array}{l}1 \\
2 \\
3 \\
4 \\
5\end{array}$ & $\begin{array}{r}62(155) \\
4(12) \\
16(34) \\
25(50) \\
0(2)\end{array}$ & $\begin{array}{r}28(97) \\
4(8) \\
17(33) \\
26(48) \\
0(2)\end{array}$ & $\begin{array}{r}164(594) \\
9(32) \\
13(37) \\
14(61) \\
3(8)\end{array}$ & $\begin{array}{r}25(259) \\
30(133) \\
8(31) \\
0(1) \\
0(13)\end{array}$ & $\begin{array}{r}115(337) \\
282(1309) \\
2(23) \\
0(2) \\
2(10)\end{array}$ & $919(3,291)$ \\
\hline 4 & $\begin{array}{l}1 \\
2 \\
3 \\
4 \\
5\end{array}$ & $\begin{array}{r}152(350) \\
2(17) \\
23(57) \\
52(98) \\
0(7)\end{array}$ & $\begin{array}{r}21(68) \\
4(8) \\
27(53) \\
42(107) \\
0(4) \\
\end{array}$ & $\begin{array}{r}198(1000) \\
9(37) \\
15(59) \\
17(83) \\
1(25) \\
\end{array}$ & $\begin{array}{r}311(1240) \\
54(186) \\
11(23) \\
6(29) \\
0(23)\end{array}$ & $\begin{array}{r}102(294) \\
426(1668) \\
2(10) \\
1(14) \\
0(17)\end{array}$ & $1,476(5,477)$ \\
\hline 5 & $\begin{array}{l}1 \\
2 \\
3 \\
4 \\
5\end{array}$ & $\begin{array}{r}84(257) \\
3(14) \\
20(59) \\
44(106) \\
1(6)\end{array}$ & $\begin{array}{r}45(129) \\
3(14) \\
17(63) \\
44(105) \\
O(3)\end{array}$ & $\begin{array}{r}184(554) \\
13(53) \\
27(94) \\
21(62) \\
1(21)\end{array}$ & $\begin{array}{r}148(414) \\
50(190) \\
15(34) \\
17(58) \\
2(20)\end{array}$ & $\begin{array}{r}164(581) \\
416(1254) \\
12(38) \\
11(39) \\
1(21)\end{array}$ & $1,343(4,189)$ \\
\hline 6 & $\begin{array}{l}1 \\
2 \\
3 \\
4 \\
5\end{array}$ & $\begin{array}{r}58(140) \\
3(4) \\
23(57) \\
40(110) \\
1(1) \\
\end{array}$ & $\begin{array}{r}14(39) \\
3(11) \\
14(48) \\
55(95) \\
1(0)\end{array}$ & $\begin{array}{r}173(638) \\
5(20) \\
7(43) \\
7(43) \\
1(12) \\
\end{array}$ & $\begin{array}{r}70(215) \\
37(181) \\
0(9) \\
1(3) \\
0(10) \\
\end{array}$ & $\begin{array}{r}88(349) \\
199(865) \\
4(12) \\
1(2) \\
1(18)\end{array}$ & $816(2,025)$ \\
\hline 7 & $\begin{array}{l}1 \\
2 \\
3 \\
4 \\
5\end{array}$ & $\begin{array}{r}39(138) \\
2(9) \\
11(38) \\
46(114) \\
1(6) \\
\end{array}$ & $\begin{array}{r}9(30) \\
2(5) \\
7(19) \\
17(53) \\
0(1)\end{array}$ & $\begin{array}{r}133(639) \\
5(29) \\
14(38) \\
8(42) \\
2(16) \\
\end{array}$ & $\begin{array}{r}87(295) \\
18(74) \\
16(34) \\
5(19) \\
1(14) \\
\end{array}$ & $\begin{array}{r}198(714) \\
247(850) \\
15(64) \\
3(20) \\
0(27) \\
\end{array}$ & $886(3,288)$ \\
\hline 8 & $\begin{array}{l}1 \\
2 \\
3 \\
4 \\
5\end{array}$ & $\begin{array}{r}100(285) \\
9(25) \\
18(62) \\
45(105) \\
1(7) \\
\end{array}$ & $\begin{array}{r}36(68) \\
1(4) \\
18(62) \\
48(102) \\
1(4) \\
\end{array}$ & $\begin{array}{r}276(1030) \\
19(84) \\
13(51) \\
12(68) \\
4(26)\end{array}$ & $\begin{array}{r}121(416) \\
53(335) \\
21(62) \\
2(8) \\
0(24)\end{array}$ & $\begin{array}{r}197(674) \\
346(1579) \\
16(63) \\
3(7) \\
2(27) \\
\end{array}$ & $1,371(5,150)$ \\
\hline 9 & $\begin{array}{l}1 \\
2 \\
3 \\
4 \\
5\end{array}$ & $\begin{array}{r}61(138) \\
3(5) \\
31(48) \\
43(107) \\
0(2)\end{array}$ & $\begin{array}{r}34(50) \\
0(8) \\
19(61) \\
40(109) \\
0(5)\end{array}$ & $\begin{array}{r}287(833) \\
3(21) \\
9(41) \\
12(38) \\
1(12)\end{array}$ & $\begin{array}{r}125(374) \\
12(42) \\
12(38) \\
1(15) \\
0(8)\end{array}$ & $\begin{array}{r}275(827) \\
148(577) \\
8(42) \\
2(19) \\
0(12)\end{array}$ & $1,128(3,432)$ \\
\hline
\end{tabular}

Source: 1000 NTACS tape trom U. S. Bureau of the Cenaus to Oak Fidge Nettonal Laboration (July 1001). 


\section{APPENDIX B}

\section{MAP OF FOUR CENSUS REGIONS AND NINE CENSUS DIVISIONS}

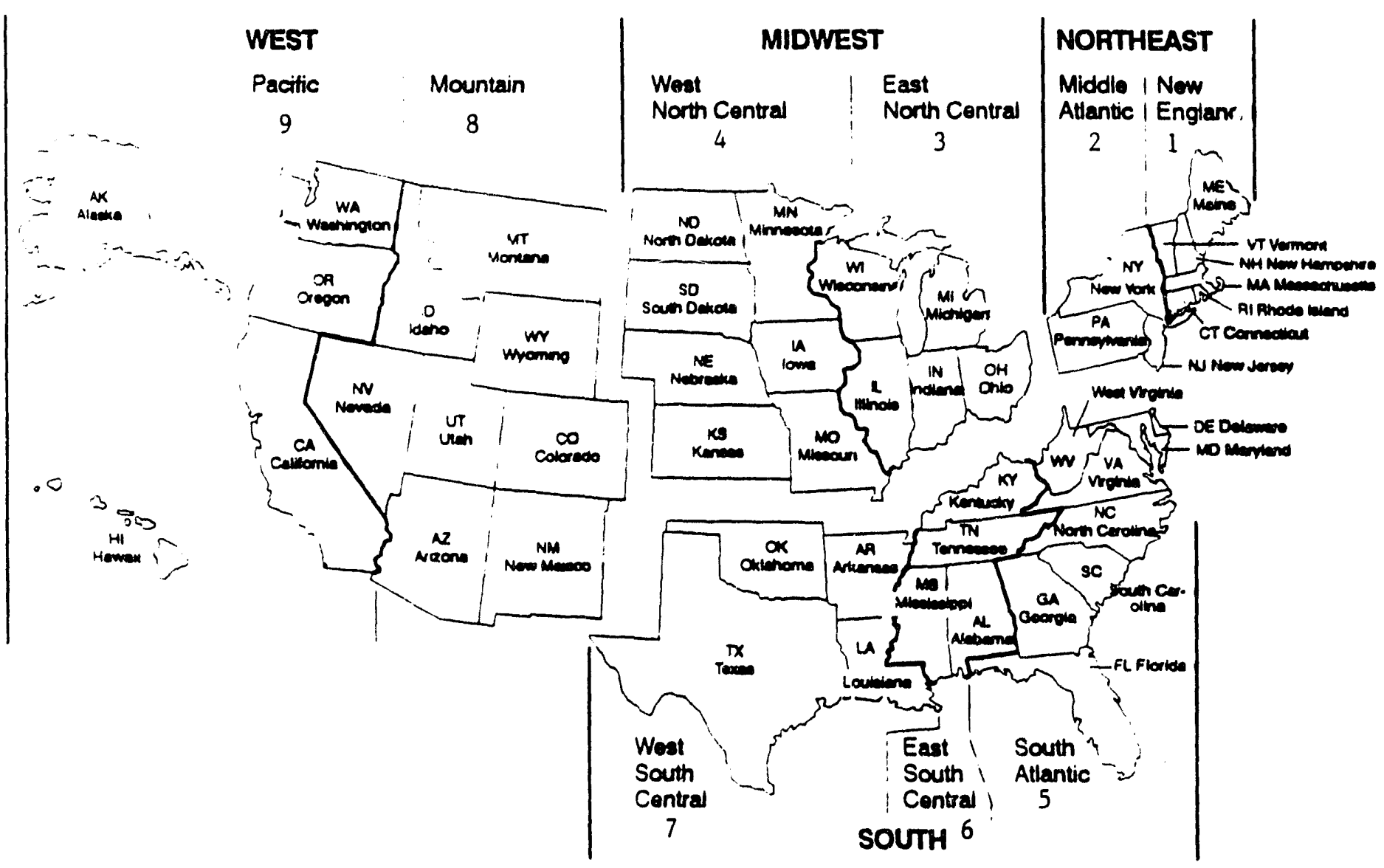

B - 1 


\section{APPENDIX C}

\section{THE 1990 NTACS-1 QUESTIONNAIRE}

This section contains a copy of the 1990 NTACS-1 questionnaire. Not all NTACS reported data are included in the NTACS Public Use File mainly due to high item nonresponse rates.

Some other data items such as "current home base state" have been suppressed or aggregated for confidentiality reasons.

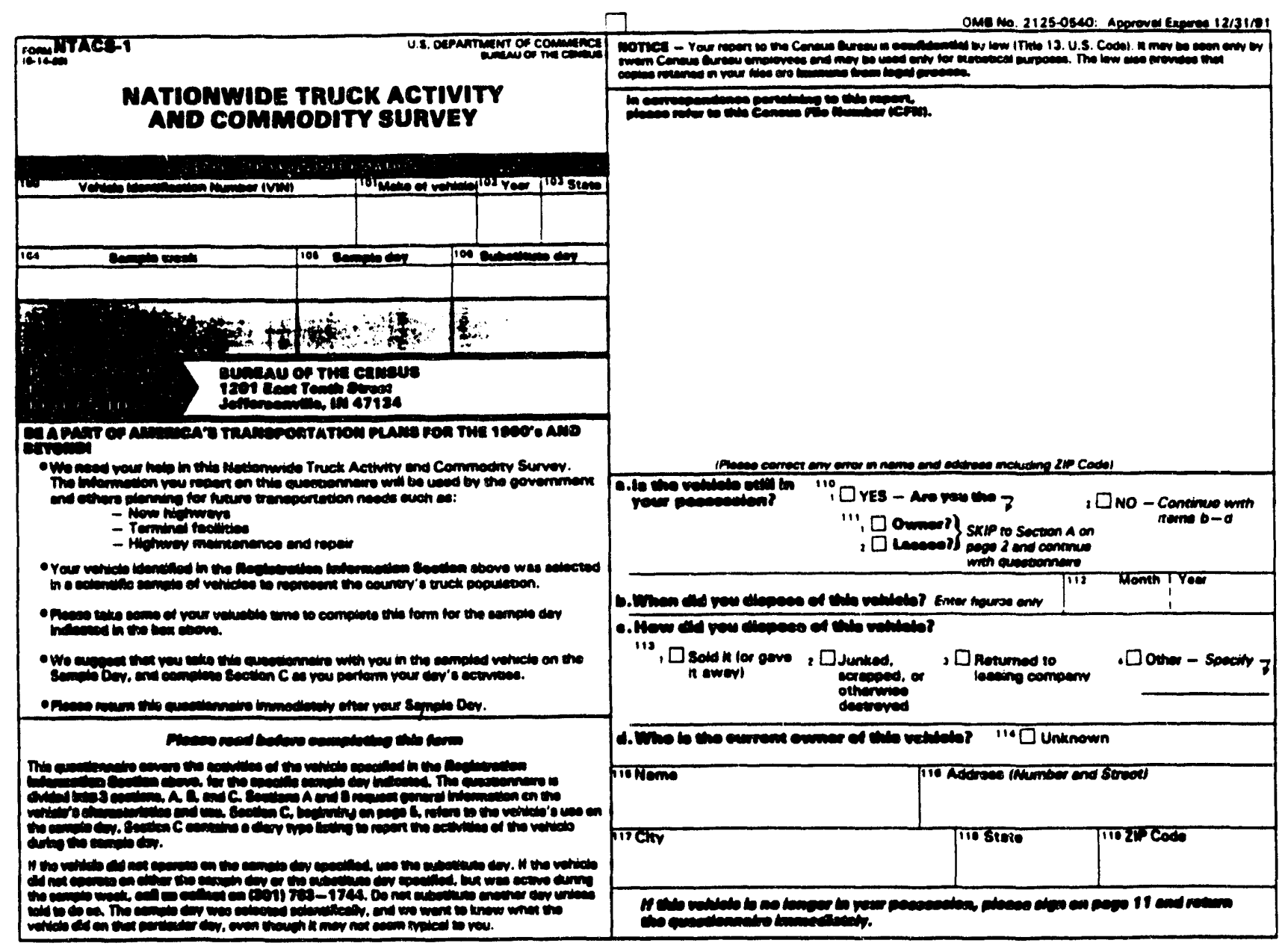



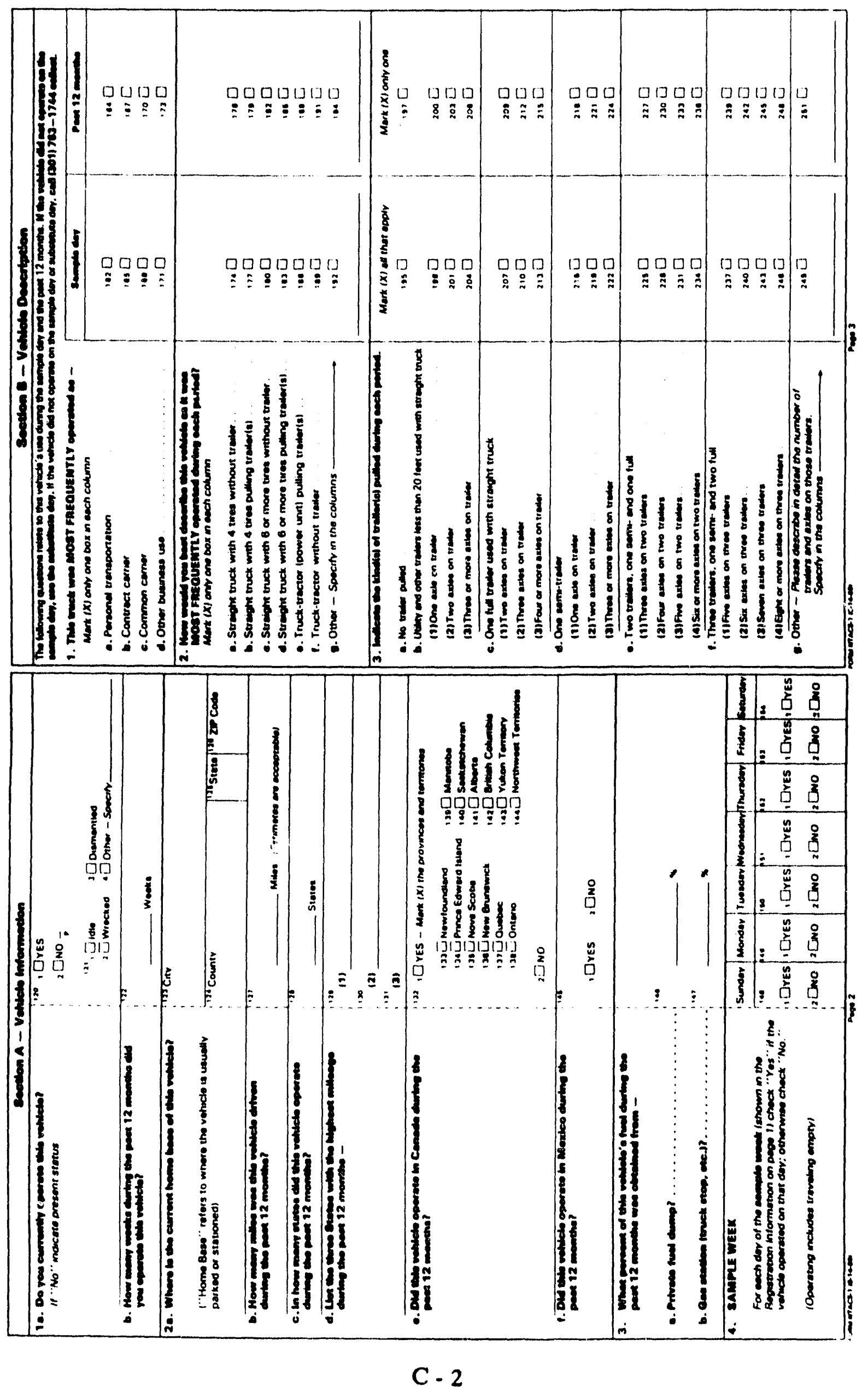

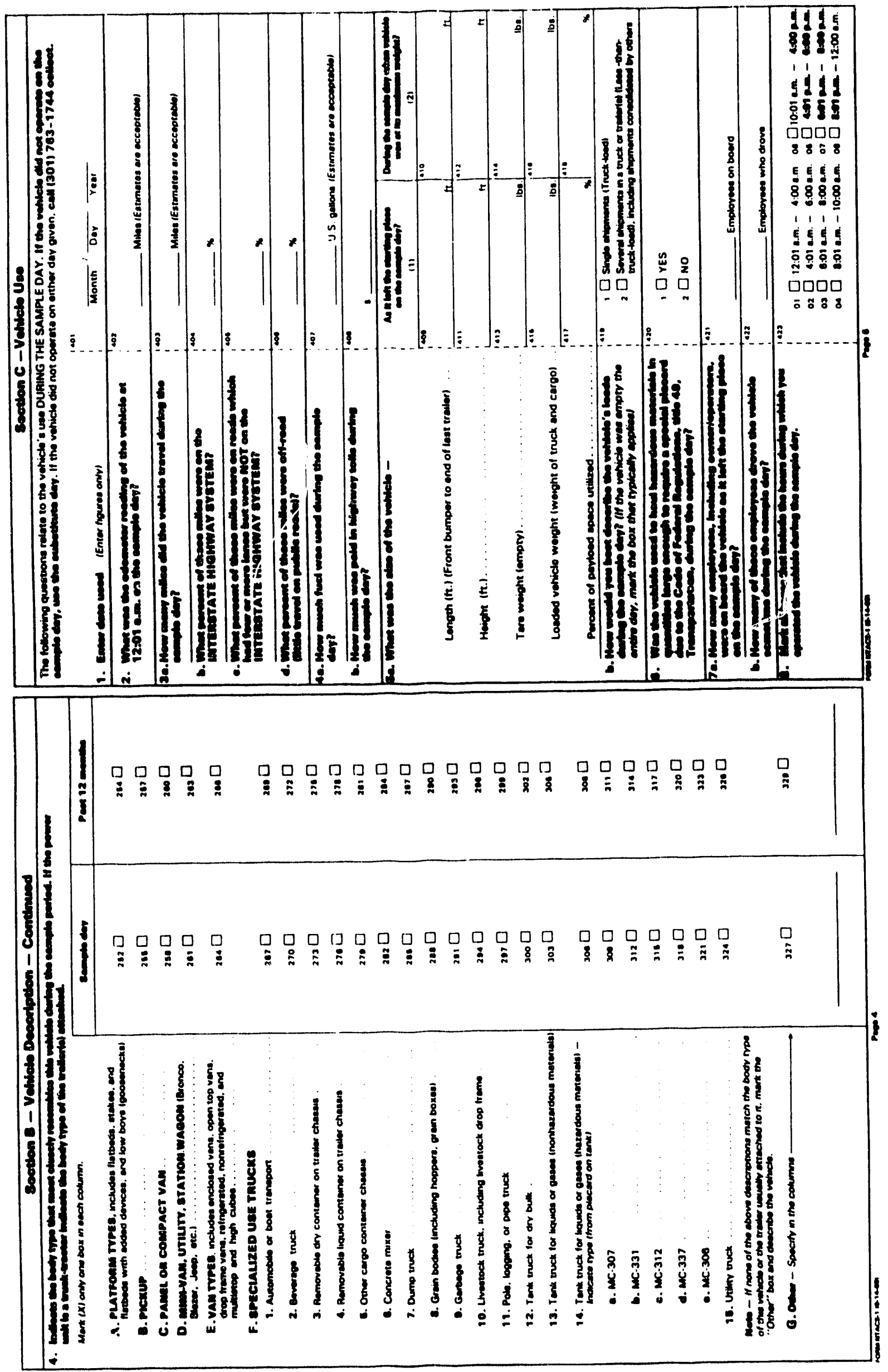

C - 3 


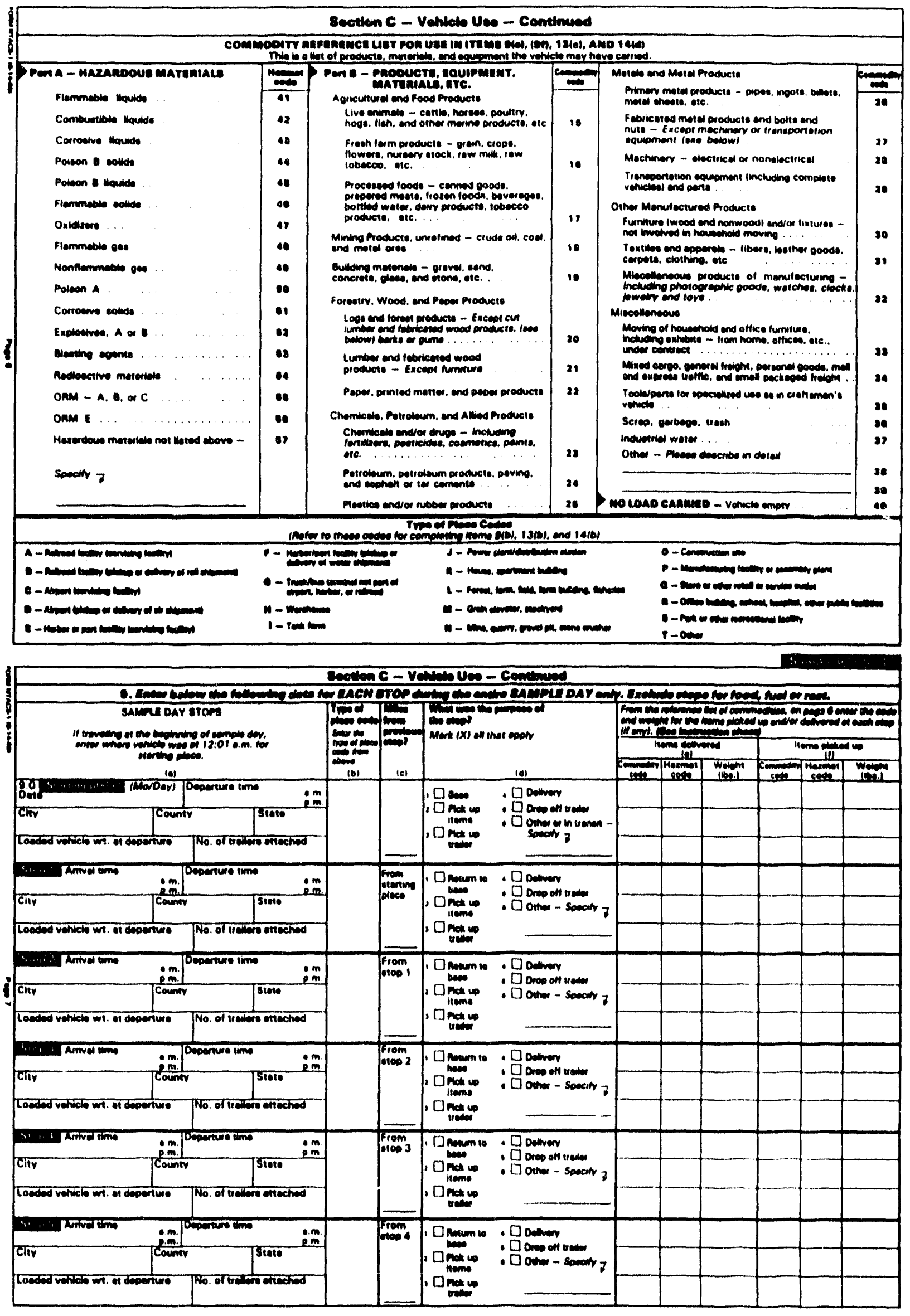




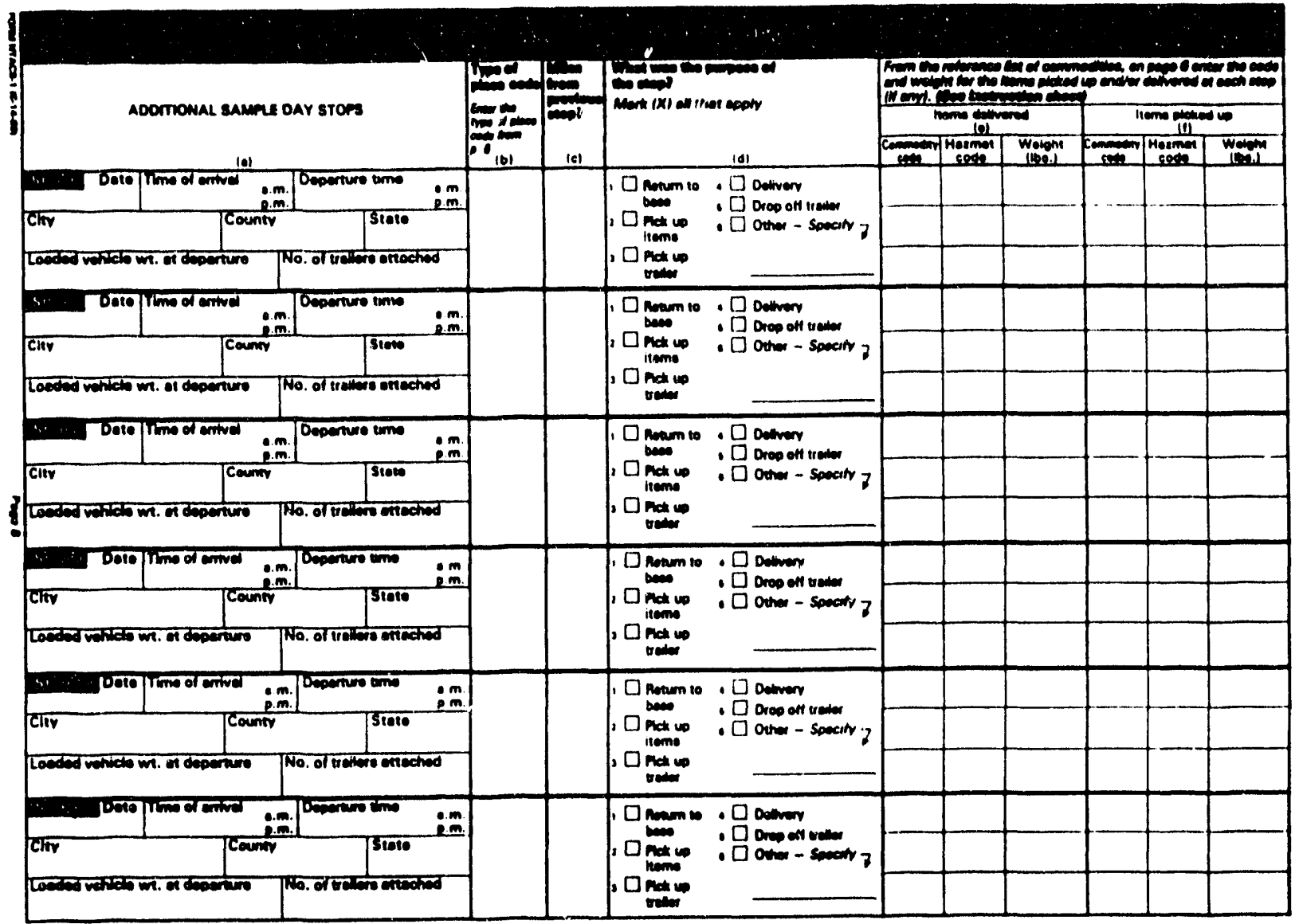

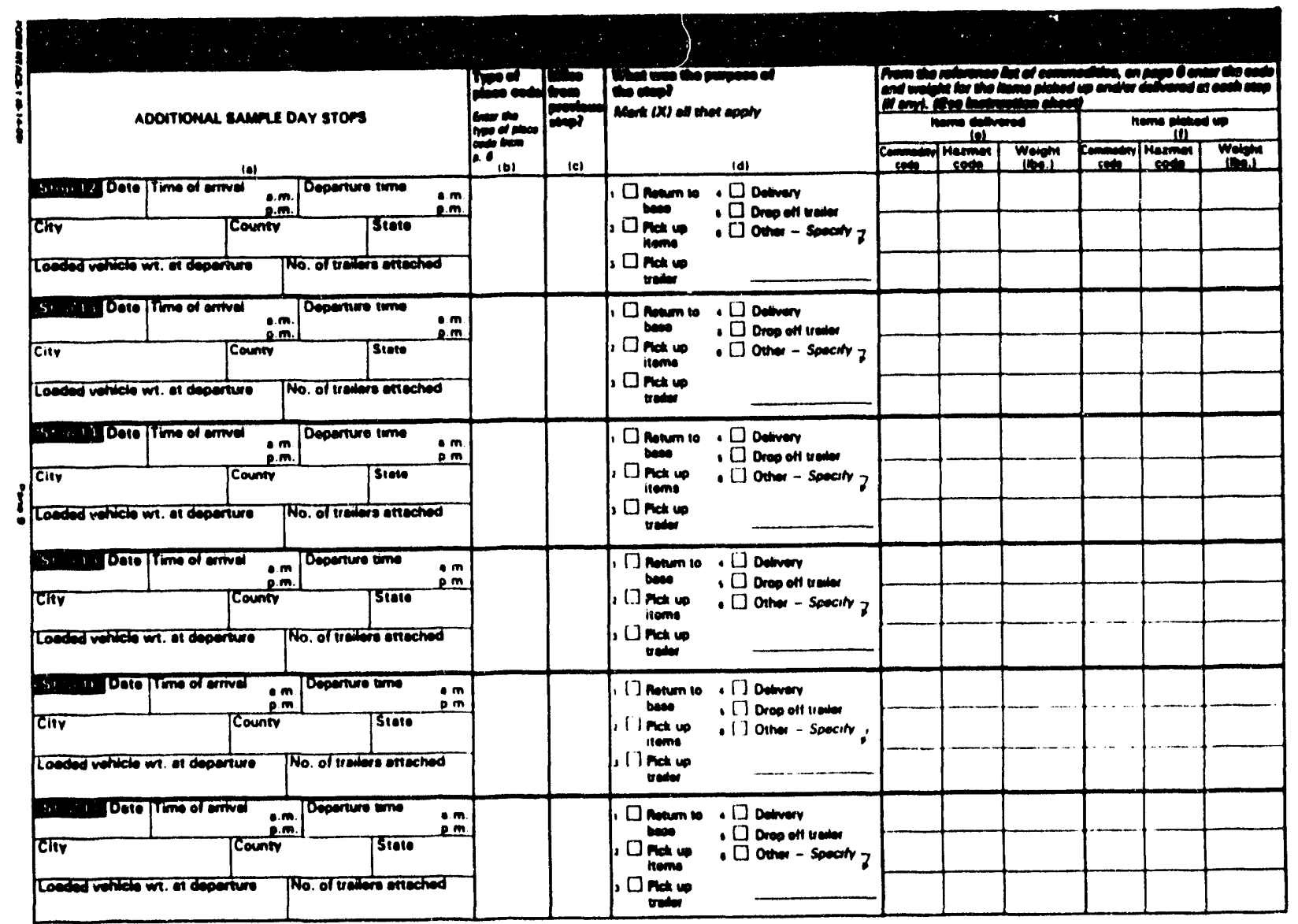

C. 5 

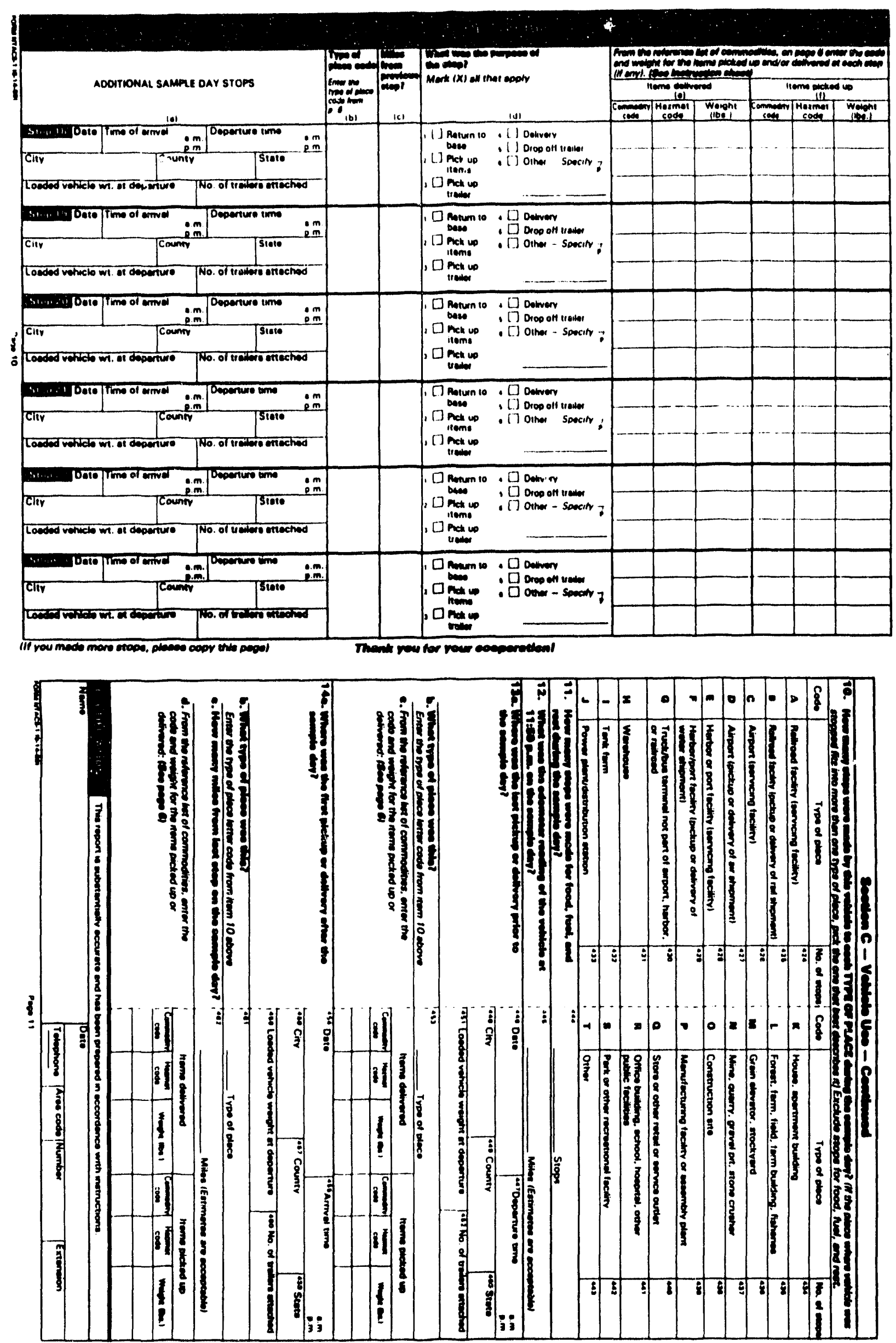

C. 6 


\section{INTERNAL DISTRIBUTION}

1. M. S. Bronzini

2. S. C. Davis

3. D. L. Gruene

4. P. S. Hu

5. M. A. Kuliasha

6. T. Wright
7. ORNL Patent Office

8-9. Central Research Library

10. Document lideference Section

11-12. Laboratory Records

13. Laboratory Records - RC

\section{EXTERNAL DISTRIBUTION}

14. D. R. Bohi, Director, Energy and Natural Resources Division, Resources for the Future, 1616 P Street, N.W., Washington, DC 20036

15. T. E. Drabek, Professor, Department of Sociology, University of Denver, Denver, Colorado 80208-0209.

16. C. D. MacCracken, President, Calmac Manufacturing Corporation, 101 West Sheffield Avenue, P.O. Box 710, Englewood, New Jersey 07631

17. J. B. Shrago, Director, Office of Technology Transfer, 405 Kirkland Hall, Vanderbilt University, Nashville, Tennessee 37240

18. G. F. Sowers, Senior Vice President, Law Companies Group, Inc., 114 Townpark Drive, Suite 250, Kennesaw, Georgia 30144-5599

19. C. M. Walton, Paul D. and Betty Robertson Meek Centennial Professor and Chairman, Department of Civil Engineering, College of Engineering, The University of Texas at Austin, Cockrell Hall, Suite 4.2, Austin, Texas 78712

20-27. Center for Transportation Analysis, Energy Division, 5500A, MS-6366, Room A217

28-29. OSTI, U.S. Department of Energy, P.O. Box 62, Oak Ridge, TN 37831

30. Office of Assistant Manager of Energy Research and Development, DOE/ORO, P.O. Box 2001, Oak Ridge, TN 37831-8600 

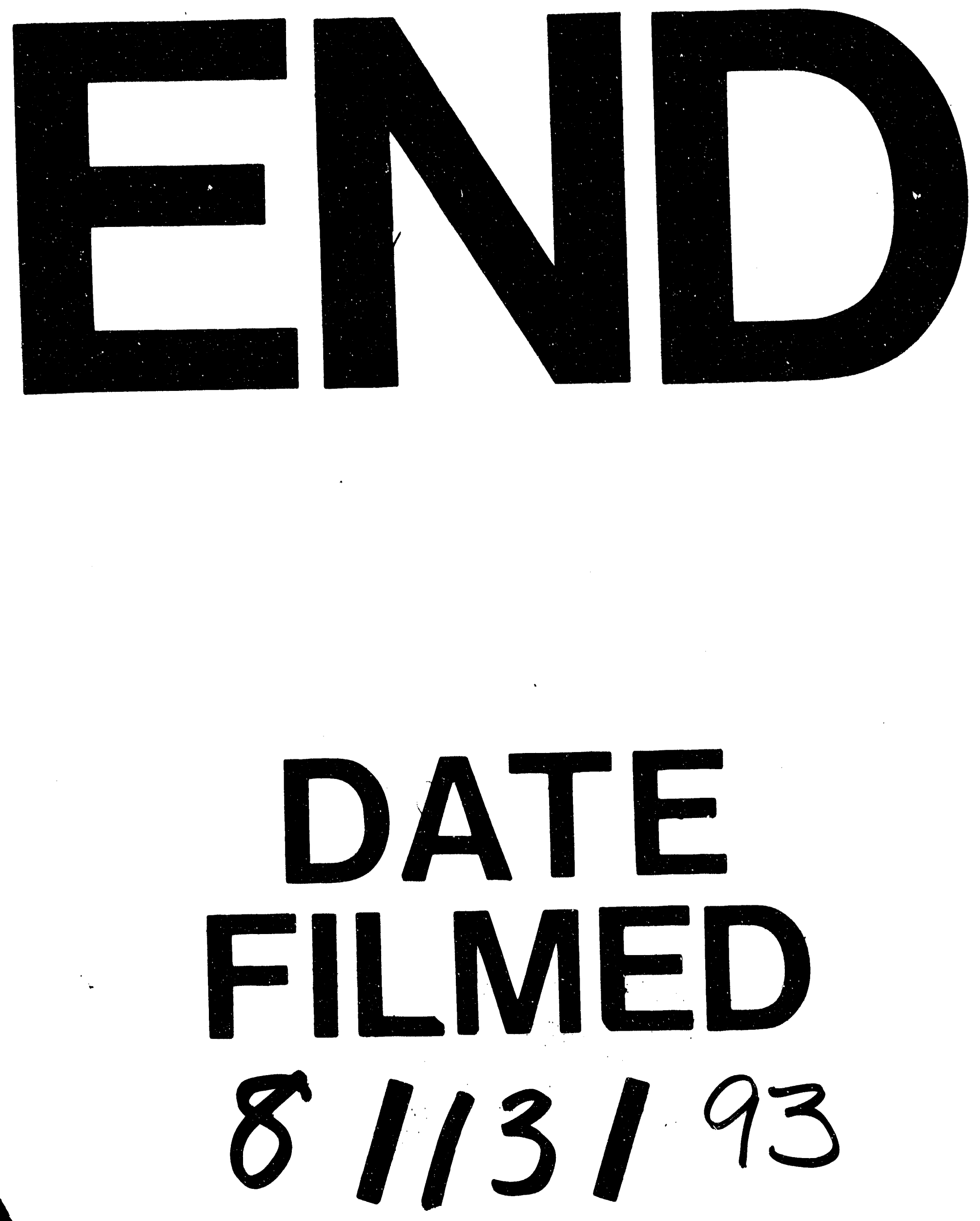